\title{
Psychological morbidity and facial volume in HIV lipodystrophy: quantification of treatment outcome
}

\section{Lisa Nelson}

MBChB with Commendation (Glasg), MRCS (Ed)

Thesis submitted for Doctor of Medicine

The University of Edinburgh 


\title{
Table of Contents
}

\author{
Acknowledgements
}

Declaration

Abstract

Chapter 1 Introduction

\subsection{Hypotheses}

\subsection{Overview of HIV Lipodystrophy}

1.2.1. Definition

1.2.2. Prevalence

1.2.3. Clinical features

1.2.3.1. Morphological features

1.2.3.2. Metabolic features

1.2.4. Pathogenesis and associated risk factors

1.2.4.1. Protease Inhibitors

1.2.4.2. Nucleoside/ non-nucleoside reverse transcriptase inhibitors

1.2.4.3. Nutritional status, age and adiposity

1.2.4.4. HIV Infection

1.2.4.5. Female Sex

1.2.4.6. Cytokines

1.2.5. Management of HIV Lipodystrophy

1.2.5.1. Non-Surgical Treatment of HIV Lipodystrophy

1.2.5.2. Surgical Treatment of HIV Lipodystrophy

\subsection{Treatment Options for Facial Lipoatrophy}

1.3.1. Autologous fat injections

1.3.1.1. History of Autologous Fat Transfer

1.3.1.2. Theories of fat graft survival

1.3.1.3. Histological evaluation of transplanted fat

1.3.1.4. Surgical technique and modifications 
1.3.1.5. Anaesthesia of donor site

1.3.1.6. Choice of donor site

1.3.1.7. Harvesting method

1.3.1.8. Method of refinement

1.3.1.9. Placement

1.3.1.10. Post-operative care

1.3.1.11. Complications

1.3.1.12. Evidence for the use of autologous fat in HIV lipodystrophy

1.3.2. Dermis-fat graft

1.3.3. Rhytidectomy

1.3.4. Temporary soft tissue fillers

1.3.4.1. Collagen

1.3.4.2. Calcium hydroxylapatite

1.3.4.3. Hyaluronic acid

1.3.4.4. Poly-L-lactic acid

1.3.5. Semi-permanent and permanent soft tissue fillers

1.3.5.1. Silicone oil

1.3.5.2. Polyalkylamide

1.3.5.3. Polymethylmethacraylate

1.3.6. Implants

1.3.7. Flaps

\subsection{Measures of psychological outcome}

1.4.1. Psychological aspects of HIV lipodystrophy

\subsection{Three Dimensional Imaging}

1.5.1. 3-D Imaging Methods

1.5.1.1. Anthropomentry

1.5.1.2. Facial Casts

1.5.1.3. 3-D Cephalometry

1.5.1.4. Morphoanalysis

1.5.1.5. 3-D Facial Morphometry

1.5.1.6. Electromagnetic Digitisation

1.5.1.7. 3-D CT Scanning 
1.5.1.8. MRI

1.5.1.9. Moire topography and contour photography

1.5.1.10. 3-D Laser scanning

1.5.1.11. 3-D Ultrasound

1.5.1.12. Stereophotogrammetry

1.5.2. Facial Volume Measurement

1.5.3. DI3D Facial Camera

1.5.3.1. Description of DI3D Facial Camera system

1.5.3.2. Principles of stereophotogrammetry

Chapter 2. Surgical technique and outcome following treatment for HIV facial lipoatrophy

2.1. Aim

2.2. Method

2.2.1. Design

2.2.2. Sample size

2.2.3. Ethical approval

2.2.4. Recruitment

2.2.5. Data collection

2.2.6. Treatment of patients with HIV lipodsytrophy

2.2.6.1. Autologous fat transfer

2.2.6.2. Newfill

2.2.6.3. Bio-alcamid

2.2.7. Clinical follow-up

2.2.8. Patient satisfaction

2.2.9. Statistical analysis

\subsection{Results}

2.3.1. Baseline characteristics of treatment groups

2.3.2. Complications of treatment

2.3.2.1. Bio-alcamid 


\subsubsection{Newfill}

2.3.2.3. Autologous fat transfer

2.3.3. Patient satisfaction with treatment

\subsection{Discussion}

2.4.1. Baseline characteristics of treatment groups

2.4.2. Complications of treatment

2.4.3. Patient satisfaction

\subsection{Conclusions}

\section{Chapter 3. Three-dimensional facial volume analysis in HIV lipodystrophy}

\subsection{Aims}

\subsection{Method}

3.2.1. Design

3.2.2. Sample

3.2.3. Outcome measures

\subsection{DI3D Facial Capture System}

3.3.1. Data capture

3.3.1.1. Calibration

3.3.1.2. Subject capture

3.3.1.3. Data processing

3.3.2. DI3D View Software

3.3.2.1. Alignment

3.3.2.2. Volume measurements

\subsection{Statistical analysis}




\subsection{Validation of the DI3D Facial Camera System}

3.5.1. Experiment 1: Measurement Bias

3.5.1.1. Aim

3.5.1.2. Method

3.5.2. Experiment 2: Re-Alignment and Re-Measurement

3.5.2.1. Aim

3.5.2.2. Method

3.5.3. Experiment 3: Test-Retest Reliability

3.5.3.1 Aim

3.5.3.2. Method

3.5.4. Statistical Analysis

3.5.4.1. Experiment 1

3.5.4.2. Experiment 2

3.5.4.3. Experiment 3

3.5.5. Results

3.5.1. Experiment 1

3.5.2. Experiment 2

3.5.3. Experiment 3

3.5.6. Discussion and Conclusions

3.5.6.1. Experiment 1

3.5.6.2. Experiment 2

3.5.6.3. Experiment 3

\subsection{Results}

3.6.1. Data completion

3.6.2. 3-D volume measurement

\subsection{Discussion}

3.7.1. Data completion

3.7.2. 3-D Facial volume results

\subsection{Conclusions}




\section{Chapter 4. Evaluation of psychological morbidity in HIV lipodystrophy}

\subsection{Aims}

\subsection{Method}

4.2.1. Design

4.2.2. Control groups

4.2.2.1. Selection of control groups

4.2.3. Psychological measures

4.2.3.1. Selection of tools for the study

4.2.3.2. Derriford Appearance Scale

4.2.3.3. Hospital Anxiety and Depression Scale

4.2.3.4. Evaluation of selected tools in HIV lipodysotrophy patients

4.2.4. Recruitment

4.2.4.1. HIV-seropositive with lipodystrophy (HIV LD) group

4.2.4.2. HIV-seropositive without lipodystrophy (HIV non LD) group

4.2.4.3. HIV-seronegative men who have sex with men (MSM) group

4.2.5. Data collection

4.2.5.1. HIV LD cohort

4.2.5.2. Control groups

4.2.6. Statistical analysis

4.2.6.1. Case-control study

4.2.6.2. Prospective study of HIV LD treatment groups

\subsection{Results}

4.3.1. HIV LD cohort versus control groups

4.3.1.1. Demographic data

4.3.1.2. Derriford Appearance Scale

4.3.1.3. Hospital Anxiety and Depression Scale

4.3.2. Psychological outcome in HIV LD treatment cohort

4.3.2.1. Data completion

4.3.2.2. Psychological outcome in HIV LD cohort as a whole 
4.3.2.3. Psychological outcome in each HIV LD treatment group 4.3.2.4. Correlation between psychological outcome and facial volume change

4.3.2.5. Clinical grading of facial lipoatrophy and correlation with psychological measures

\subsection{Discussion}

4.4.1. Selection of control groups

4.4.2. Psychological characteristics of cohort versus control groups at baseline and confounding variables

4.4.3. Psychological measures

4.4.3.1. Selection of psychological measures

4.4.3.2. Comparison of study results with published clinical trials using assessment tools

4.4.4. Psychological outcome in HIV LD treatment group

4.4.4.1. Data completion

4.4.4.2. DAS and HADS scores

4.4.4.3. Correlation between psychological outcome, facial volume and clinical grading

\subsection{Conclusions}

\section{Chapter 5. DISCUSSION}

5.1. Limitations of the case control study

5.2. Limitations of the prospective study

5.3. Comparison of treatment results and directions for future research

\section{Chapter 6. CONCLUSIONS}

6.1. HIV LD treatment cohort versus control groups

6.2. Prospective study of HIV LD treatment group

\section{Bibliography}

\section{Appendices}


Appendix 1. Patient and physician assessment of lipodystrophy

Appendix 2. Patient Satisfaction Scales

Appendix 3. Linear mixed effect regression models

Appendix 4. Derriford Appearance Scale

Appendix 5. Hospital Anxiety and Depression Scale

Appendix 6. Reference qualitative interview questions

Appendix 7. Presentations arising from this thesis

Appendix 8. Publications arising from this thesis

\section{Figures}

1.1. DI3D Facial Capture System

1.2. Images captured from each pod

1.3. Principle of stereophotogrammetry

1.4. Cloud point images

1.5. Solid, shaded and wireframe models

2.1. Fat harvest from the periumbilical region

2.2. Centrifuged fat

2.3. Fat injection technique

2.4. Bio-alcamid syringes

2.5. Bio-alcamid injection technique

2.6. Sinus of right cheek

2.7. Post-operative scarring following removal of Bio-alcamid

2.8. Extrusion of Bio-alcamid

2.9. Migration of Bio-alcamid

2.10. Summary of Newfill treatment sessions

2.11. Pre-operative and 6-month post-operative result for patient treated with fat 
2.12. Pre-operative and 6-month post-operative result for patient treated with Newfill

2.13. Pre-operative and 6-month post-operative result for patient treated with Bio-alcamid

3.1. Landmarks for image alignment

3.2. 3-D backplane

3.3. Facial cast with and without placement of malar silicone mould

3.4. Image alignment

3.5. Measurement bias by true volume (top) and separately for each face (bottom)

3.6. Measurement error, either with or without realignment of images

3.7. Bland-Altman plot for repeated measurements of forehead, cheek and nose of ten individuals, taken one week apart

3.8. Change in measured 3-D facial volume for each treatment group

3.9. Percentage of injected volume remaining at follow-up

4.1. Flow diagram of Study Design

4.2. Summary of DAS-24 male participant scores versus population reference values

4.3. Boxplots (median, quartiles and range) of HADS-D and HADS-A scores for all subgroups

4.4. Summary of psychological measures of all HIV LD patients undergoing treatment for facial lipoatrophy

4.5. Box plot of DAS-24 results for each treatment group over time

4.6. HADS-A results for each treatment group over time

4.7. HADS-D results for each treatment group over time

\section{Tables}

1.1. Clinical features of HIV-associated lipodystrophy 
1.2. Therapeutic options for HIV-associated lipodystrophy and related metabolic complications

2.1. Demographics of autologous fat, Newfill and Bio-alcamid treatment groups

2.2. Clinical patterns of HIV-associated lipodystrophy in patients referred for treatment.

2.3. Summary of complications associated with the use of Bio-alcamid® in the Edinburgh Unit

2.4. Summary of Newfill sessions

2.5. Patient satisfaction scores

3.1. Estimated variances of measurements made with or without realignment of images, their ratio, with $95 \% \mathrm{CI}$, and p-value from F-test of equality of variances

3.2. Number of patients treated and follow-up 3-D data completed

3.3. Injected Volumes (Bio-Alcamid and AFT groups only), comparing those with or without follow-up volume measurements

3.4. Number of revision procedures or re-treatments performed

3.5. Mean, SD and number of observations of volume injected or volume relative to baseline

3.6. Correlations between number of treatments and volume changes over time for NewFill patients

4.1. Demographics of HIV LD, HIV non-LD and MSM subgroups

4.2. Summaries of DAS-24 scores by subgroup with p-values from ANOVA tests

4.3. Summaries of HADS-D and HADS-A scores by subgroup with p-values from ANOVA tests

4.4. Number of patients treated and follow-up psychological data completed

4.5. Baseline DAS-24, HADS-D and HADS-A scores, comparing those with or 
without follow-up measurements

4.6. $\mathrm{p}$-values for tests of no difference between groups in mean value or mean change over baseline using ANOVA

4.7. Correlations between changes in volume and changes in psychological factors

4.8. Patient vs. Physician Grading of severity

4.9. Psychological Scores vs. Patient Grading of severity

4.10. Psychological Scores vs. Physician Grading of severity 


\section{Acknowledgements}

I would like to thank my supervisor and mentor, Mr Ken Stewart, who provided the inspiration to start this project and for his continual guidance and support throughout. Most of all I would like to thank him for his belief in me and encouragement which has been completely invaluable.

I would also like to thank my co-supervisors, Mr David Soutar and Professor Michael Sharpe, for their advice and support.

I am indebted to the Medical Photography Department at St. John's Hospital for assisting with 3-D imaging of patients, and preparation of material for publication and presentation. In particular I would like to thank Mark Darling for his help during validation of the system and for his enormous effort and patience in transporting the system to Glasgow on a regular basis.

I am extremely grateful for the help of Alex McConnachie in analysis of results, preparation of this manuscript and for his prompt response to my often last minute demands on his time.

I am thankful to the Plastic Surgery out-patient clinic and secretarial staff in both St John's Hospital and Glasgow Royal Infirmary for their help in the smooth running of my research clinics. I am especially grateful to Mrs Valerie Will for her help in the organisation of clinics and theatre lists and her persistent communication with patients on my behalf.

I would like to acknowledge the excellent treatment of patients with Newfill by Tanya Brandon at St. John's Hospital and Pat McGuiness and Laura Mathers at the Brownlee centre, Glasgow. 
I am very grateful to Jeff Miller and the prosthetists at St John's hospital for their skilful production of facial casts and silicone moulds for use in the validation studies. The recruitment of patients for the project would have been impossible without the efforts of the medical and out-patient clinic staff at the Brownlee Centre and Sandiford Centre, Glasgow and the Genito-Urinary Medicine and Infectious Diseases Departments in Edinburgh. In particular I would like to thank Dr Dan Clutterbuck, Dr Andrew Seaton, Dr Andrew Winter, Dr Ray Fox and Dr Gordon Scott for their support and help with the project.

For advice on choice of psychological measures I am very grateful to Dr Anne Tait, and Dr Timothy Moss.

I am indebted to Dimensional Imaging, Glasgow for development of 3-D software for this project. I would like to emphasis my particular thanks to Colin Urquart for his support and advice, to Douglas Green for his technical support and to Anders Sundstedt for his help with 3-D animation.

I would like to acknowledge my huge appreciation to the funding charities who made this project possible including: The Henry Smith Charity, the William Rooney Foundation, the Sick Kids Foundation, Edinburgh and NHS Lothian Endowments. Finally, I am so thankful to my family for their endless support and belief in me. 


\section{Declaration}

This thesis has been composed by myself. It has not been accepted in any

previous application for a degree. The work was carried out by myself at St.

John's Hospital, Livingston and Canniesburn Plastic Surgery, Glasgow Royal

Infirmary, Glasgow. Material published from this thesis was approved by the

lead supervisor. All sources of information have been specifically

acknowledged.

Signed

Lisa Nelson, 2012 


\section{Abstract}

\section{Introduction}

HIV-associated facial lipoatrophy is a stigmatizing condition associated with significant psychological morbidity. The condition may be treated with soft tissue fillers, although quantification of objective and patient-reported outcomes is lacking. The primary aim of this study was to evaluate change in facial volume and psychological morbidity following treatment for HIV lipodystrophy with autologous fat transfer, Newfill and Bio-alcamid. An additional aim of the study was to compare psychological characteristics between HIV seropositive patients with lipodystrophy (HIV LD) and without lipodystrophy (HIV non-LD) and HIV seronegative men who have sex with men (MSM).

\section{Methods}

HIV LD patients were treated with autologous fat, Newfill or Bio-alcamid based on a clinical assessment in a prospective, observational study. The Colemnan technique of fat transfer was utilised. Newfill injections were carried out at monthly intervals using 1 vial per cheek. Bio-alcamid was injected subcutaneously under aseptic technique to achieve the desired cheek augmentation. 3-D images were obtained pre-operatively then at 2,6 and 12 months post-operatively using the DI3D system. Volume changes in treated areas were measured using DI3D software. The DAS-24 and HADS were used to assess psychological morbidity at similar time intervals.

An additional case-control study was conducted to measure psychological morbidity in HIV LD, HIV non-LD and MSM groups utilising the Derriford Appearance Scale (DAS-24) and Hospital Anxiety and Depression Scale (HADS).

Data was analyzed using appropriate statistical tests. 


\section{Results}

ANOVA tests demonstrated significantly higher DAS-24 scores in the HIV-LD group compared to the HIV non-LD and MSM groups. No difference in HADS-A scores was seen between groups.

48 patients with HIV LD were treated: 16 patients had Bio-alcamid augmentation, 20 patients received Newfill injections and 12 patients underwent fat transfer. The mean injected volume of Bio-alcamid was $25.5 \mathrm{cc}$ The Wilcoxin test demonstrated no significant difference in mean volume change relative to zero at 2, 6 and 12 months. The mean injected volume of fat was $20.1 \mathrm{cc}$, which did not differ from the measured volumes at 2 months $(\mathrm{p}=0.15)$. There was a mean reduction in measured volume of $7.3 \mathrm{cc}$ at 6 months and $9 \mathrm{cc}$ at 12 months $(\mathrm{p}<0.001)$.

For Newfill, the mean volume change compared to baseline was $8.7 \mathrm{cc}$ at $2 \mathrm{months}$, increasing to $12.6 \mathrm{cc}$ at 6 months and $12.3 \mathrm{cc}$ at 12 months.

ANOVA tests demonstrated no difference in psychological outcomes between groups. There was a significant improvement in DAS-24 scores compared too baseline for all 3 groups throughout follow-up. For Bio-alcamid, a significant improvement in HADS-A and HADS-D scores were seen at 2 months but mean scores increased at 6 and 12 months. In the fat group, some improvement in HADS-A and HADS-D scores were seen at follow-up, although values only reached significance at 6 months. In the Newfill group, small improvements in both HADS-A and HADS-D were demonstrated in the post-treatment scores, although these did not reach significance. No correlation between change in facial volume and psychological measures was demonstrated.

\section{Conclusions}

The case control study demonstrated that patients with HIV lipodystrophy have greater 
distress relating to body image and depression compared to HIV-seropositive patients without lipodstrophy and HIV-seronegative MSM control groups.

The prospective study of the HIV LD treatment cohort demonstrated a change in 3-D measured facial volume for all 3 groups. Bio-alcamid produced the greatest, permanent volume change but was associated with the most complications. Newfill was associated with a moderate, delayed volume augmentation but was insufficient for some patients with severe lipoatrophy. Volume enhancement with autologous fat was good immediately post-operatively but variable degrees of fat resorption occurred. Treatment was associated with improved body image perception. However, initial improvements in anxiety and depression symptoms were not maintained in the long term. 


\section{CHAPTER 1 Introduction}

\subsection{Hypotheses}

The hypotheses of this study were that:

1. HIV lipodystrophy is associated with psychological morbidity relating to body image, anxiety and depression.

2. Treatment of HIV lipodystrophy using autologous fat transfer, Newfill@ or Bioalcamid $\AA$, is associated with an improvement in psychological morbidity and anatomical volume of treated areas.

3. The DI3D system is a valid and reproducible method of recording and measuring facial 3-D volume.

\subsection{Overview of HIV Lipodystrophy}

HIV-associated lipodystrophy is a syndrome of morphological changes (central fat accumulation and peripheral fat atrophy) and metabolic changes (hyperlipidaemia and insulin resistance) which has been increasingly reported in patients since 1998 (Carr, Samaras et al. 1998). The consequence of altered body fat morphology is social stigmatization that may lead to poorer adherence and the failure of anti-retroviral therapy. Moreover, metabolic abnormalities may increase the risk of cardiovascular disease (Carr, Samaras et al. 1999).

\subsubsection{Definition}

At least five definitions of lipodystrophy have been proposed (Carr, Miller et al. 2000; Martinez, L et al. 2000; Saint-Marc, Partisani et al. 2000), and although HIV investigators have attempted to develop meaningful case definitions for changes in 
body fat that can be used for surveillance purposes, the reprodtucilbillity off such definitions has not been tested.

A more detailed model has been proposed by Canr and the HfIIV ILipodtystinophy Case Definition Study Group including age, sex, durration of Hul in infectiom, IHIIV dhisease status, waist to hip ratio, anion gap, serum HIDI chollesteroll comcenturation, thumik tho peripheral fat ratio, percentage leg fatt, and intra-albdominall to extura-albdhomimall fatt ratio(Carr, Law et al. 2003). This model has a sensittivitty of $79 \% \%$ amnd speccifficitity of $80 \%$ for the diagnosis of lipodystrophy. Two mone simple modells thave allso beem dewissed for

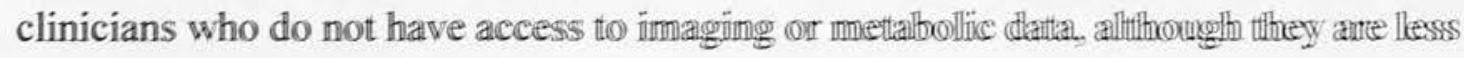
sensitive and specific than the complete model. In a sturdy to comparte three verssioms off.

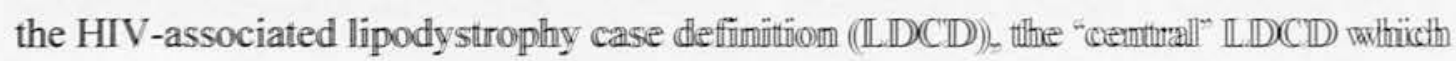

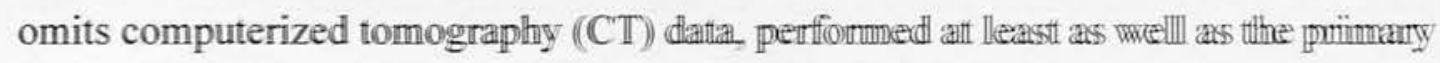
LDCD which includes all parameters, and both werte mome semsittivive titham the momimaging model (Law, Puls et all. 2006)). The most commom metthod for gradting

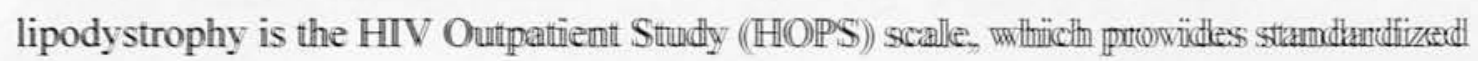
but subjective descriptions for milld, moderatte or severte lippodtysturopplmy alt multijple siites (Lichtenstein, Ward et al. 2001). Chamges are graded ass "miuldd" (motticeralble omlly iff

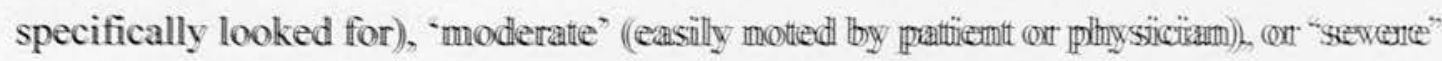
(obvious to the casual observer). The LDCCD wass froumd tho be the best cromellatue off

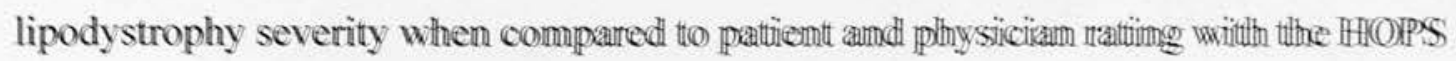

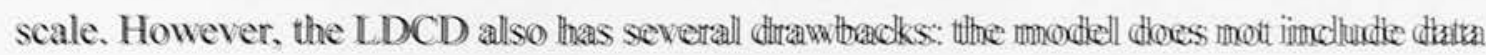

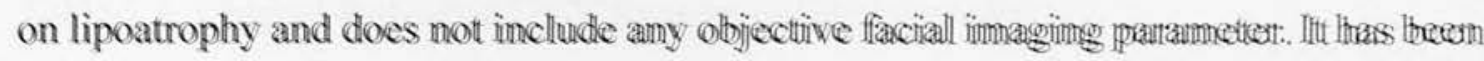
suggested that lipodystrophy should be comsidered at symdtrome off 4 or more sulb-

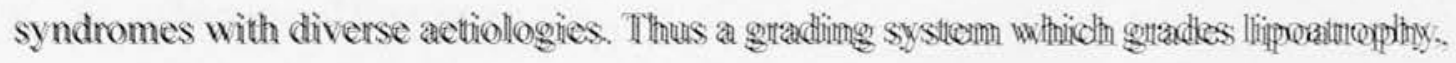

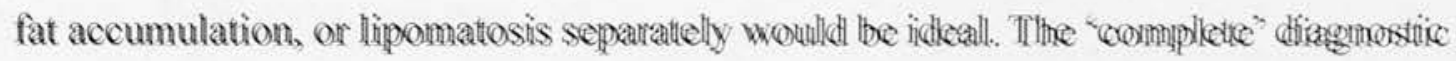

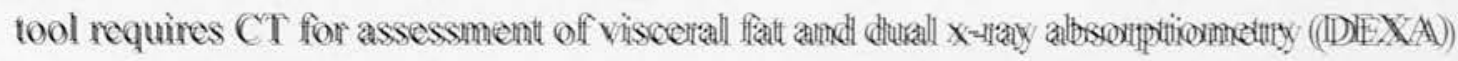


scan for assessment of extremity fat. These are not modalities available to the general HIV population for the purposes of diagnosis of HIV lipodystrophy. Given the need for rationing of resources, it may be inappropriate to perform tests which are expensive, require expertise and do not lead to a direct intervention.

\subsubsection{Prevalence}

From the era of Highly Active Antiretroviral Therapy (HAART), a spectrum of changes in body fat have been reported to occur in $20-80 \%$ of subjects receiving these therapies (Carr, Law et al. 2003; Miller, Carr et al. 2003; Sattler 2003). The wide variability in prevalence is mostly related to an absence of standardized case definitions but also inclusion of cases based on patients' self-report of changes, variability in the way different physicians clinically assess fat distribution, and extraction of data from chart reviews. In a review of 15 studies with over $100 \mathrm{HIV}$ patients who reported features of HIV-associated adipose redistribution syndrome (HARS), HARS was reported in 9-48\% patients (mean 32\%)(Lichtenstein, Wanke et al. 2004).

\subsubsection{Clinical features}

Table 1.1 summarizes the morphological and metabolic changes associated with HIVassociated lipodystrophy.

Table 1.1. Clinical features of HIV-associated lipodystrophy

\section{Morphological}

- Peripheral lipoatrophy:

Face, arms, legs, buttocks 


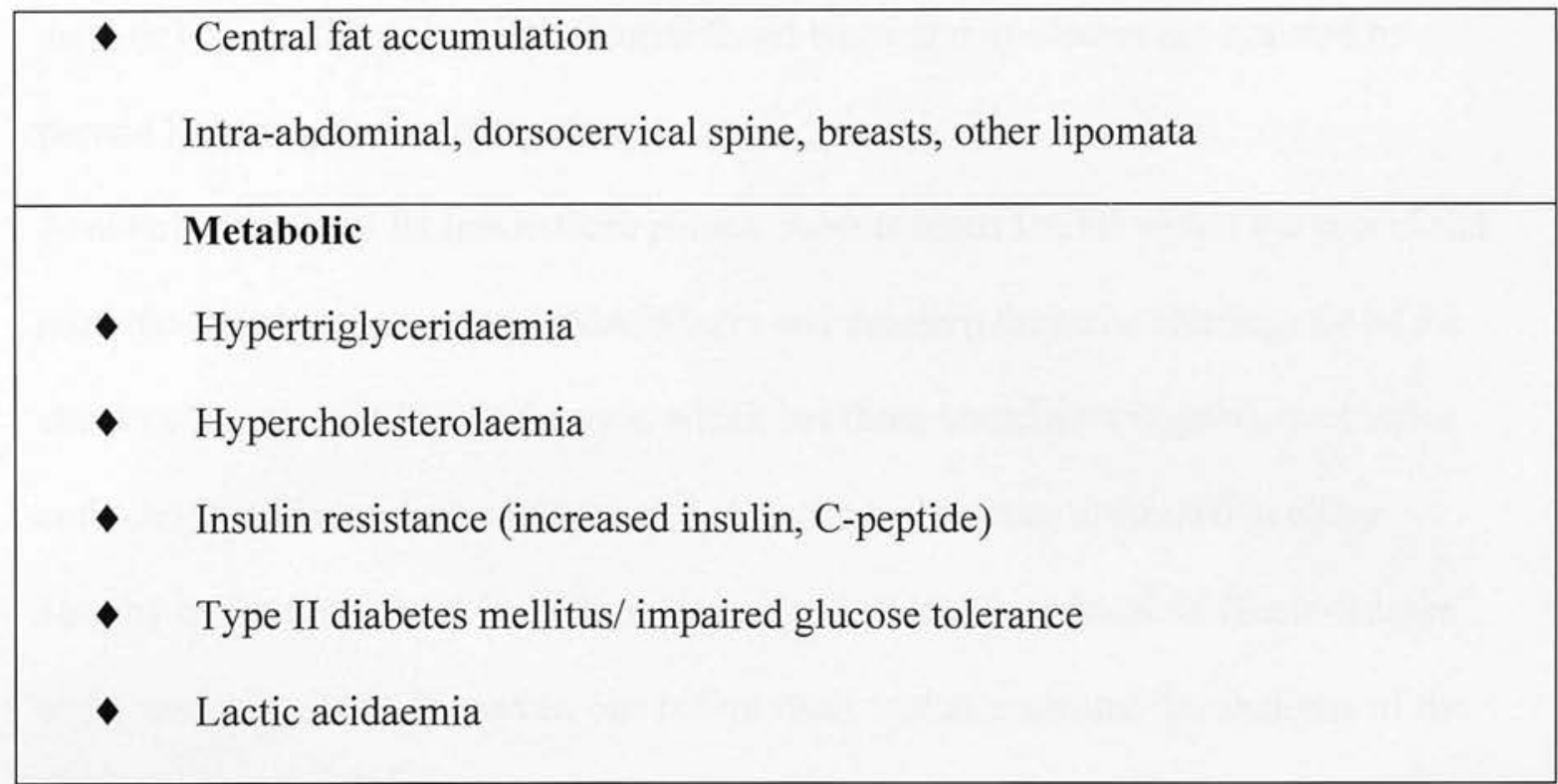

\subsubsection{Morphological changes}

Changes in body fat distribution are characteristic of HIV associated lipodystrophy.

On body shape changes alone, three different patterns of lipodystrophy have been described:

- signs of peripheral fat atrophy alone

- signs of lipohypertrophy alone

- a mixed type involving both lipoatrophy and fat accumulation

There is now accumulating evidence that lipoatrophy, central adiposity and the combination of both result from different pathogenetic developmental processes.(Behrens and Schmidt 2003)

The most prominent clinical sign is a loss of subcutaneous fat in the face and extremities. Facial atrophy characterized by sunken cheeks, hollow temples, sunken eyes, and prominent zygomatic arch has been attributed to the loss of buccal, parotid, and pre-auricular fat pads. Adipose tissue loss from the peripheral regions occasionally leads to prominent veins resembling varicosities. The most common site for lipoatrophy is the face, and is often the most distressing for the patient as it identifies 
them as being affected by HIV. Central facial wasting may also be accentuated by parotid hypertrophy in some patients.

Anatomically, facial fat lies in three planes: subcutaneous fat, fat within the superficial musculo-aponeurotic system (SMAS) layer and the deep fat pads. The deep fat of the cheek constitutes the Bichat fat sack, which has three branches: temporal, buccinator and retromandibular. In the literature, facial atrophy has been attributed to either atrophy of the deep facial fat pads, or atrophy of all layers of facial fat (Serra-Renom and Fontdevila 2004). However, one recent study which evaluated the anatomy of the buccal fat pad of Bichat in HIV-related facial atrophy reported normal position and thickness of the fat pad and suggests that the cachectic appearance of the face is likely secondary to profound atrophy of the subcutaneous tissues in these areas (Talmor, Hoffman et al. 2002).

A classification of facial lipoatrophy has been proposed to assist in treatment decisions (James, Carruthers et al. 2002). Grade 1 is mild and localized, and the appearance is almost normal. Grade 2 has longer and deeper central cheek atrophy, with the facial muscles (especially zygomaticus major) beginning to show through. In grade 3 , the atrophic area is even deeper and wider with the muscles clearly showing and in grade 4 , atrophy covers a wide area and extends up towards the orbit.

Some patients have concomitant deposition of excess adipose tissue around the neck, over the dorsocervical spine (buffalo hump), upper torso and intra-abdominal region (Talmor, Hoffman et al. 2002). Dorsocervical fat accumulation can be disfiguring and is associated with the development of neck pain and sleep apnoea (Piliero, Hubbard et al. 2003). Fat accumulation at the occipital region may also occur. Breast enlargement has been reported in both sexes, although it is unclear whether this is due to excess subcutaneous fat, glandular hypertrophy or both.(Paech, Lorenzen et al. 2002) 
Other possible related symptoms include dry skin or lips, nail dystrophy, hair loss or brittleness, dysmenorrhoea, impotence, avascular osteonecrosis, osteoporosis, liver steatosis, cardiovascular disease, and increased bleeding in haemophiliacs (Carr and Cooper 2000). Visceral fat accumulation has also been reported to cause distension and abdominal cramps. A few cases of thrombosis and pancreatitis have been reported related to high triglyceride levels.

Several techniques are suitable for measuring regional fat distribution including dual energy x-ray absorptiometry (DEXA), computer tomography (CT), magnetic resonance imaging (MRI) and ultrasound. Anthropometric measurements, including waist circumference, sagittal diameter, and skin fold thickness are cheaper and easier to perform than imaging techniques but are operator dependent.(Behrens and Schmidt 2003) Three-dimensional laser scans have been used for measurement of facial volumes (Ras, Habets et al. 1996; Hajeer MY 2002) and may provide an objective tool for monitoring changes in facial lipoatrophy (Benn, Ruff et al. 2003).

Sonography has been used in HIV lipodystrophy to quantify subcutaneous fat(Martinez, L et al. 2000), and in intervention studies to quantify facial tissue thickness changes (Valantin, Aubron-Olivier et al. 2003; Moyle, Lysakova et al. 2004). Martinez et al measured subcutaneous fat thickness at three reference skin points (periumbilical, brachial, and malar) and intra-abdominal fat thickness in HIV-1infected patients with and without lipodystrophy and in healthy controls. Measurement of subcutaneous facial and arm fat was simpler, less variable, and more discriminative to diagnose abnormal regional fat distribution than that of intra-abdominal fat. They concluded that sonography was useful in quantifying regional fat distribution in HIV-1infected patients. Its potential advantages include simplicity, availability, safety, patient tolerance, and low cost compared with DEXA, computed tomography, and MRI.

However, the use of ultrasonography to measure malar fat is not advocated by Carey et 
al who assessed facial lipoatrophy changes in HIV-infected adults receiving antiretroviral therapy who participated in a 48-week, randomized, placebo-controlled trial of rosiglitazone.(Carey, Wand et al. 2005) Ultrasound was performed at weeks 0 , 24 and 48 to determine the subcutaneous fat thickness over the malar bone. No significant correlation was found between between malar sonographic measures and body composition parameters assessed by DEXA and CT. They conclude that sonography may be subject to measurement error, correlates poorly with more established objective measures of lipoatrophy, and is therefore not recommended. However, these studies compare facial ultrasound with DEXA and CT measurements of non-facial body parts. There are no studies to compare the efficacy of facial ultrasound and other imaging modalities in the assessment of HIV-associated facial lipoatrophy. These studies also demonstrated the need for non-invasive technology to quantify facial lipoatrophy.

\subsubsection{Metabolic changes}

Frequently, complex metabolic alterations are associated with the described body shape alterations. These include peripheral and hepatic insulin resistance, impaired glucose tolerance, type II diabetes, hypertriglyceridaemia, hypercholesterolaemia, increased free fatty acids, and reduced high density lipoproteins.(Sekhar, Jahoor et al. 2002) The prevalence of insulin resistance and glucose tolerance is reported in the literature at 20$50 \%$ depending on study design and measurement methods (Carr, Samaras et al. 1999).

\subsubsection{Pathogenesis and associated risk factors}

The pathogenesis of HIV lipodystrophy is complex and the aetiology is likely to be multi-factoral. Published studies provide evidence for two assumptions: firstly, lipoatrophy and lipoaccumulation result from divergent mechanisms. Secondly, the 
various classes of anti-retroviral drugs contribute to the lipodystrophy syndrome by different and probably overlapping mechanisms.

\subsubsection{Protease Inhibitors}

Many studies have suggested a link between the use of protease inhibitors and the development of lipodystrophy. Carr and Cooper performed two studies and found that $64 \%$ of patients receiving protease inhibitor (PI) therapy developed lipodystrophy, compared to $3 \%$ of protease inhibitor-naïve patients.(Carr and Cooper 2000) One year later, Carr et al reported $83 \%$ of subjects receiving PI therapy developed lipodystrophy, while only $4 \%$ of those not receiving PI therapy developed lipodystrophy.(Carr, Samaras et al. 1999) They estimated that the mean time to lipodystrophy was 10 months after commencing protease inhibitor treatment. An increased duration of PI therapy has been shown to increase the odds of fat wasting and the probability of intraabdominal fat accumulation.(Carr, Samaras et al. 1998) Protease inhibitors account for the majority of metabolic abnormalities associated with the lipodystrophy syndrome. Numerous studies report increases in the levels of total triglycerides and triglyceriderich lipoproteins (VLDL) accompanied by raised LDL levels after initiation of PI therapy. These changes are frequently associated with hyperinsulinaemia and/ or insulin resistance (Walli, Herfort et al. 1998).

The underlying mechanisms for fat depletion and accumulation in HIV-infected patients receiving antiretroviral therapy remain unclear. One hypothesis proposes that protease inhibitors bind to and inhibit proteins involved in lipid metabolism. (Carr, Samaras et al. 1998) There is homology between the amino acid sequences of the catalytic site of HIV protease and two proteins involved in lipid metabolism regulation: low-density lipoprotein receptor-related protein (LRP) and cytoplasmic retinoic-acid binding protein type 1 (CRABP-1). Protease inhibitor binding of LRP reduces postprandial chylomicron clearance which leads to hyperlipidaemia. Binding of 
protease inbibitors to $\mathrm{CRABP}-1$ prevents binding of retinoic acid which diminishes the production of cis-9-retinoic acid. This reduces differentiation and increases apoptosis of peripheral adipocytes and leads to impaired fat storage and lipid release. An alternative suggested mechanism is that PI therapy inhibits adipogenesis by a process involving sterol regulatory element binding proteins (SREBPs), which are membrane bound transcription factors that play a central role in cellular lipid homeostasis. (Caron, Auclair et al. 2001)

Differences in the metabolic activity of central, dorso-cervical and peripheral adipocytes has been proposed. Thus, the impaired fat storage and hyperlipidaemia associated with protease inhibitor treatment may lead to preferential central accumulation of fat. A link between PI therapy and altered apolipoprotein (B and C-III) synthesis has been postulated.(Carr, Samaras et al. 1998)

\subsubsection{Nucleoside/ non-nucleoside reverse transcriptase inhibitors}

Some have suggested that the real risk of developing lipodystrophy is associated with nucleoside reverse transcriptase inhibitors (NRTI) therapy, not PI therapy. NRTIs are phosphorylated and incorporated into new DNA, resulting in HIV DNA termination. Mitochondrial toxicity associated with non-nucleoside reverse transcription inhibitors has been well established and is thought to contribute to the fat redistribution syndrome seen in patients with HIV infection (McComsey and Walker 2004). It is thought that the toxicity of these drugs is due to inhibition of mitochondrial DNA polymerase gamma resulting in impaired synthesis of mitochondrial enzymes that generate ATP. (Hadigan, Miller et al. 1999) Decreased levels of mitochondrial DNA were found in the blood cells of NRTI-treated patients (Cote, Brumme et al. 2002). Depletion of mtDNA and structural changes in the mitochondria are thought to result in increased rates of apoptosis in subcutaneous adipocytes. The resulting apoptosis or complement (C3) deficiency can lead to abnormal fat distribution. 
Several retrospective cohort studies demonstrate an association between HIV lipodystrophy and NRTIs, most notably stavudine (Carr, Miller et al. 2000). Exclusive NRTI therapy seems to have minor impact on lipid metabolism. Although fat redistribution has been observed in patients treated with NRTIs alone, the addition of a PI to the regimen substantially increases the risk of lipoatrophy.(Madge, Kinloch-deLoes et al. 1999) It has been suggested that the pattern of lipodystrophy varies with drug therapy: NRTIs are associated with lipoatrophy alone, whilst NRTI combined with PI therapy is associated with lipoatrophy and lipohypertrophy. However, the wide variety of drug combinations used make it difficult to identify risk associated with particular drugs. Carr et al demonstrated no significant difference in physical or body composition parameters between patients with NRTI-lipodystrophy syndrome and those with protease inhibitor-associated lipodystrophy syndrome. However, NRTIlipodystrophy syndrome had more recent onset of symptoms and weight loss, higher lactate and alanine aminotransferase, and lower albumin, cholesterol and triglycerides, glucose and insulin compared to protease inhibitor-associated lipodystrophy syndrome.(Carr, Samaras et al. 1999)

\subsubsection{HIV infection}

The development of HIV lipodystrophy has been positively associated with the duration of HIV infection, negatively associated with previous HIV viral load, and both positively and negatively associated with blood CD4 lymphocyte counts in various studies.(Chen, Misra et al. 2002)

\subsubsection{Nutritional status, age and adiposity}

Body adiposity before receiving antiretroviral therapy may also affect features of lipodystrophy. In one cross sectional study, patients with Body Mass Index (BMI) greater than $28 \mathrm{~kg} / \mathrm{m} 2$ had higher prevalence of buffalo hump and breast enlargement, but a lower prevalence of facial and gluteal fat loss compared with underweight 
patients (BMI <20 kg/m2).(Pettit, Kotler et al. 1999) Older people tend to have greater body fat mass, particularly intraabdominal fat, which may contribute to the body fat changes seen in HIV lipodystrophy.(Mercie, Tchamgoue et al. 1999)

\subsubsection{Female sex}

Trunk obesity rather than subcutaneous fat wasting appears to be more common in women than in men (Hadigan, Miller et al. 1999) although prospective studies are required to evaluate whether there are true differences between the sexes.

\subsubsection{Cytokines}

There is an association between lipodystrophy and levels of serum inflammatory factors. One study analyzed the mRNA expression of adipocytokines and transcriptional factors in fat samples from 26 patients with peripheral lipoatrophy.(Jan, Cervera et al. 2004) The patients' fat showed higher values of apoptosis, fibrosis, vessel density and macrophage infiltration than the controls' fat, together with lower adiponectin and leptin mRNA levels and higher interleukin (IL)-6 and tumour necrosis factor (TNF)alpha mRNA levels. Elevated levels of the soluble type 2 tumour necrosis factor-alpha receptor has been linked with insulin resistance associated with lipodystrophy. C-peptide has been shown to be the strongest metabolic predictor of future LD severity in patients receiving combines NRTI and protease inhibitor therapy.(James, Carruthers et al. 2002)

\subsubsection{Management of HIV Lipodystrophy}

\subsubsection{Non-surgical management}

To date, there has been no adequate treatment to resolve lipodystrophy. Various therapeutic options are summarized in table three. Dietary modification is ineffective. Exercise may lead to partial beneficial decrease in central fat accumulation and triglyceride levels but at the expense of increased peripheral fat wasting(Milinkovie and 
Martinez 2005). The use of recombinant growth hormone can be beneficial in patients with visceral abdominal fat or dorso-cervical fat pad. However, it is not recommended for the treatment of patients with features of lipoatrophy as it may lead to a reduction in peripheral fat. The impact of switching anti-retrovirals that are implicated in the lipodystrophy syndrome has been commonly assessed in different studies. In general, switching antiretroviral regimes may improve metabolic abnormalities but the impact on body fat is very small.

Table 1.2. Therapeutic options for HIV-associated lipodystrophy and related metabolic complications

Lifestyle changes (reduce saturated fat and cholesterol intake, increase physical activity, cessation of smoking)

- Change anti-retroviral therapy

$\checkmark \quad$ Statins

$-\quad$ Fibrates

- Metformin

- Recombinant human growth hormone

- Surgical intervention

\subsubsection{Surgical management of HIV lipodystrophy}

There are various described surgical techniques for the treatment of lipodystrophy and these can be divided into treatments for lipoatrophy or treatments for lipohypertrophy and have been summarized in our paper entitled Plastic surgical options for HIVassociated lipodystrophy' (Nelson and Stewart 2008) In this section, the treatment options for this study shall be described, namely: autologous fat injections, Newfill ${ }^{\text {, }}$ 
Bio-alcamid® and liposuction. The published literature on other plastic surgery options for HIV lipodystrophy shall also be summarized.

\subsection{Treatment Options for Facial Lipoatrophy}

The surgical techniques described for HIV-associated facial lipoatrophy include; autologous fat transfer, dermis-fat grafts, rhytidectomy, flaps, soft tissue fillers and implants.

\subsubsection{Autologous fat transfer}

The technique of soft tissue augmentation with centrifuge prepared autologous fat grafting has been developed by Coleman and is now being used in the treatment of HIV-related facial lipoatrophy. (Coleman 2004; Serra-Renom and Fontdevila 2004) Autologous tissue is the ideal tissue filler for physiological reasons. The potential advantages include biocompatibility, versatility, stability, and a natural appearance. (Niechajev and Sevcuk 1994) The Coleman technique involves harvesting fat by aspiration, which is then centrifuged and re-injected to augment areas of facial wasting. Longevity of correction depends on the tissue, mobility and vascularity of the recipient site. The various techniques for fat transfer and controversies surrounding technique shall be discussed in this section.

\subsubsection{History of Autologous fat transfer}

Autologous transplantation of adipose tissue is a technique that has gained increasing popularity over the past two decades. However, the concept of fat transfer is not new. The earliest recorded human free fat transfer was by Neuber in 1893, who reported using upper extremity fat to recontour soft-tissue facial defects, and emphasised the importance of small grafts for more predictable results (Neuber 1893). This was followed by reports of free fat autographs to fill soft-tissue defects by Czerny (1895), 
Lexer (1910), and Rehn (1912). In 1911, Bruning was the first to inject autologous fat into the subcutaneous tissue for the purpose of soft tissue augmentation. In 1912, Hollander published photographs showing the results of fat infiltration into two patients with lipoatrophy of the face (Hollander 1910). In 1926, Miller wrote about his experiences with infiltration of fatty tissue through cannulas. Early optimism was tempered by Peer, who demonstrated histologically that fat grafts lost approximately 45 percent of their weight and mass one year or more after transplantation (Peer 1956). Interest in autologous fat transfer diminished until the advent of liposuction surgery in the 1980s which provided plastic surgeons with semi-liquid fat which could be grafted with relative ease. In the 1980 s, Illouz and Fournier developed an approach to fat transfer by syringe harvesting called 'microlipoinjection'(Illouz 1985; Fourneir 1986). Initial experimentation with this new technique yielded variable results. In the early 1990s, Ersek reported disappointing results, with fat loss ranging from $20-90$ percent, which generated a widespread negative perception of the technique(Ersek 1991). Since the mid 1990s, Coleman has confirmed the efficacy and permanence of grafted fat, but stresses that this is dependent on the harvesting and grafting technique adopted (Coleman 2001). Indeed, ongoing variability in results has been reported depending on choice of technique, the area treated, patient factors and surgical experience. In recent years, many different techniques have evolved and a standard procedure that is adopted by all practitioners has not yet been developed.

\subsubsection{Theories of fat graft survival}

There have been two major theories proposed for the survival of fat grafts. The 'host cell replacement theory' was based on the work of Neuhof and Hirshfeld in 1923. It postulated that transplanted fat undergoes complete cell death and that histiocytes would scavenge lipid material and eventually replace all the host tissue. The more 
popular theory in recent years is the cell survival theory, which states that some of the graft adipose tissue survives after the host reaction subsides and is based on the work of Peer. The graft of adipose tissue goes through an initial period of ischaemia and obtains nutrition through plasmatic imbibition. Circulation is restored to the grafted fat cells in a manner similar to the revascularization of a skin graft. In the first 4 days, host cells, such as polymorphonuclear leukocytes (PMNs), plasma cells, lymphocytes, and eosinophils, infiltrate the graft. On or about the fourth day, neovascularization is evident. Histiocytes act only to remove fat from disrupted cells (Peer 1955).

More recently, the role of adipose-derived stem cells and preadipocytes in transplanted fat has been investigated. These cells appear to be more resistant to trauma than mature adipocytes due to lower oxygen consumption requirements. Some researchers have proposed that survival of adipose-derived stem cells in the stromal cell fraction of transplanted fat is a major factor and may account for the variability in survival of fat grafts between individuals.(Billings and May 1989)

\subsubsection{Histological evaluation of transplanted fat}

Multiple animal models have been proposed to study the technique of autologous fat transfer. Recent histological evaluation of transplanted fat grafts to the lips of rabbits has demonstrated an early inflammatory response to the injected fat followed by sequestration of nonviable tissue. The transplanted fat remained viable at one year, with good overall survivability and minimal fibrosis.(Brucker, Sati et al. 2008) However, there are few studies to evaluate the histologic fate of reinjected fat in humans. Niechajev et al obtained biopsies of transplanted fat to the cheeks of 9 patients with subcutaneous fat atrophy, 7 to 36 months after transplantation. Histologic analysis showed an organised lobular structure of transplanted fat at 7 months with more pronounced fibrosis between lobuli in the specimens obtained at 36 months. Niechajev 
and Sevcuk 1994) Carpaneda studied collagen alterations in adipose tissue transplanted to abdominal subcutaneous fat prior to abdominoplasty.(Carpaneda 1994) He found a type 1 collagen capsule around the grafted fat and several alterations to type 1 and type 3 collagen synthesis, degradation and remodelling. A shift in the inflammatory process from the peripheral viable region to the central unviable region was also demonstrated, where pseudocysts were present. Chajchir et al performed biopsies on the sites of grafted fat at intervals. Three months after transfer, zones of cytosteatonecrosis, lipophagic granulomas, lymphocytes, adipocytes, giant multinucleated cells, and new vessels were found. At 6-8 months, the specimens were infiltrated heavily by PMNs in a fibrotic matrix, and, at 1 year, a large amount of connective tissue and fibrotic reaction was present. Some fat was still present, but the authors felt that the inflammatory reaction may contribute more to the long-term result (Chajchir, Benzaquen et al. 1990). There is some disagreement about the role of fibrosis. Some authors believe that fibroblasts cause contraction, whilst others believe that they can provide augmentation. A fibrous host reaction may be all that occurs in some patients although it may be argued that it ultimately fulfilled the role of soft tissue augmentation.

\subsubsection{Surgical technique and modifications}

A greater understanding of how to maintain viable fat has led to modifications in technique that are believed to improve clinical results. These modifications are intended to preserve the delicate structure of adipocytes and provide a robust blood supply on which fat cells are extremely dependent. However, there is little objective scientific evidence and studies are difficult to compare as authors use different harvesting techniques, re-injection techniques and means of evaluating the outcome. 


\subsubsection{Anaesthesia of the donor site}

The use of local anaesthesia at the harvest site has been debated. Whilst some practitioners argue that vasoconstriction by local application of epinephrine may have a detrimental effect on the graft, others believe that reduced bleeding helps to maintain viability.(Sommer and Sattler 200) Some studies have also shown that lidocaine may potentially inhibit adipocyte metabolism in culture, but this effect persists only as long as the lidocaine is present and has not been demonstrated in vivo.(Moore, Kolaczynski et al. 2005) According to a recent survey of American plastic surgeons (Kaufman, Bradley et al. 2007), the majority of practitioners use a wetting solution of with a mixture of lidocaine and epinephrine (e.g. $50 \mathrm{ml} 1 \%$ lidocaine plus $1 \mathrm{ml}$ of epinephrine 1:1000 plus 1 litre of normal saline).

\subsubsection{Choice of donor site}

The most common sites for fat harvest include the abdomen, thigh, flank, gluteal region and knee. In patients with HIV associated lipodystrophy, sites of lipohypertrophy such as the dorso-cervical fat pad may also be utilised for fat harvest. When assessing central adiposity, it is important to evaluate whether the fat accumulation lies within the subcutaneous tissue or peritoneum. Abdominal computed tomography scans have shown that the increase in girth in patients with HIV lipodystrophy is largely due to fat accumulation in the peri-visceral region, which is less accessible for harvesting of fat grafts. Some authors have suggested that adipocytes from the gluteal-femoral region are larger and have greater lipogenic activity, making them the ideal choice for donor fat (Hudson, Lambert et al. 1990). However, other studies have shown no difference between choice of donor site following assessment of histological parameters (Ullmann, Shoshani et al. 2005) or adipocyte viability using cell proliferation techniques (Rohrich, Sorokin et al. 2004). Thus, the donor site may be chosen based on 
surgeon or patient preference. The solutions are infiltrated in a ratio of roughly $1 \mathrm{cc}$ of solution per cubic centimetre of fat to be harvested.

\subsubsection{Harvesting method}

Common harvesting techniques used for fat isolation include syringe aspiration and lipoaspiration. It has been hypothesized that liposuction may be more detrimental to the fat cells than syringe aspiration. However, laboratory studies comparing the techniques have reported contradictory results. Various studies have shown no difference in fat cell viability, as assessed by cell proliferation, graft weight maintenance or histological evaluations (Smith, Adams et al. 2006; Pu, Coleman et al. 2008). Some investigators argue that adipose aspirates from conventional liposuction have sub-optimal levels of cellular function despite maintenance of normal structure and numbers of viable fat cells, and therefore may not survive well after transplantation (Pu, Cui et al. 2005). The negative pressure applied using either technique appears to determine the extent of cell damage. A number of authors recommend using half the normal suction pressure to avoid mechanical injury to adipocytes during aspiration (Niechajev and Sevcuk 1994). Nguyen et al showed $90 \%$ adipocyte injury at $-760 \mathrm{mmHg}$ on histological sections compared to $5 \%$ injury after gentle syringe aspiration (Nguyen, Pasyk et al. 1990). It is estimated that the pressure when a syringe plunger is pulled, is $40 \%$ of liposuction pressures. However, it has been demonstrated that negative pressures can reach $100 \%$ of liposuction pressures when a 10-cc syringe plunger is pulled maximally (Smith, Adams et al. 2006).

The Coleman technique is now the most popular method of autologous fat transfer. It was first described in 1994 using a syringe, cannula, and centrifuge and later refined with the use of Coleman instruments (Coleman 1997). For harvesting fat, Coleman recommends a $3 \mathrm{~mm}$ diameter, 2 -hole Coleman harvesting cannula attached to a $10-\mathrm{ml}$ 
Luer-Lok syringe. Via a small stab incision, the blunt cannula is advanced and retracted through the harvest site using digital manipulation of the plunger to create gentle negative pressure. Parcels of fat are delivered to the cannula and once full, the syringe and plunger are disconnected and a plug is attached before placement in the centrifuge.

\subsubsection{Method of refinement}

There are various methods of fat refinement advocated to improve the long-term take of fat grafts. These include centrifugation, washing, rolling and treatment with other substances ranging from growth factors to growth medium (Yuksel, Weinfeld et al. 2000).

Washing liposuction aspirate with saline, Ringer's lactate or sterile water is felt by some authors to obtain the highest proportion of viable cells (Rubin and Hoefflin 2002). However, Coleman argues that washing of harvested tissue may disrupt the fragile fatty tissue architecture and also remove fibrin, thus hampering anchorage of fat to the surrounding tissues (Coleman 2002).

Centrifugation, popularised by the Coleman technique remains the most widely practised method of fat purification. The rationale for centrifugation is to remove excess blood, oil and local infiltration fluid. Thus a purified, predictable volume of fat may be re-injected. Prevention of inflammatory reaction to debris by centrifugation or washing is also an intuitive argument. Studies to evaluate adipocyte viability between centrifuged and non-centrifuged fat samples are also conflicting. One study investigating cell proliferation has shown no difference between groups although the authors acknowledge that evaluation of long-term cell viability requires an in vivo animal model (Rohrich, Sorokin et al. 2004). Further scientific studies are required to determine the best method of cell isolation whether by centrifugation or other means. 
The Coleman protocol for centrifugation involves centrifuging syringes of fat at 3000 rpm for 3 minutes, which separates the harvested material into three layers. The upper layer is composed of oil from ruptured adipocytes and should be decanted. Absorbent material can be used to wick off any remaining oil. The bottom layer is composed of blood, water and local anaesthesia, and is allowed to drain by gravity after the plug is removed. The middle layer is composed of refined fat, which is transferred to 1-cc Luer-Lok syringes for infiltration. During the transfer process, most authors favour a closed technique. Advantages include maintenance of sterility and avoidance of exposure to air, which may lead to desiccation of adipocytes.

\subsubsection{Placement}

Infiltration cannulas devised by Coleman are commonly used for placement of fat grafts. For the treatment of facial lipoatrophy, a blunt 17-gauge cannula is recommended. The cannula is inserted to the recipient tissues via $2-\mathrm{mm}$ incisions and fat is injected on withdrawal of the cannula into multiple planes. In the face, the maximum amount of fat deposited with each withdrawal should be $1 / 10^{\text {th }} \mathrm{ml}$. Placement of small parcels of fat is recommended to maximise the surface area of contact between the harvested fat and recipient site. Studies have shown that only $40 \%$ of grafted fat tissue is viable $1 \mathrm{~mm}$ from the edge of the graft at 60 days (Coleman 2002). Therefore, decreasing the diameter of the grafted fat parcels ensures adequate blood supply to central adipocytes for nutrition and respiration. Ideally, the threads of fat should not exceed $3 \mathrm{~mm}$ diameter. An additional benefit of maximising the surface area of grafted fat is increased stability and fat anchorage via host fibrin (Coleman 2002).

Although some authors believe that injection of fat graft into muscle is associated with increased viability due to improved vascularity, clinical evidence shows good survival 
in scarred tissue despite reduced vascularity in these conditions (Rigotti, Marchi et al. 2007).

Because of the tendency for fat resorption, many surgeons believe it is necessary to overcorrect the soft tissue defect. For surgeons who follow this practise, the amount of overcorrection thought to be required varies between $10-50 \%$ (Kaufman, Bradley et al. 2007).

\subsubsection{Post-operative care}

Elevation, cold therapy and external pressure with elastic tape may be used to minimize oedema although compression to the grafted area should be avoided to prevent migration of fat.

\subsubsection{Complications}

Fat grafting is considered to be a relatively safe procedure with few serious complications. The most common complications are aesthetic. Undercorrection may result from insufficient volume correction intraoperatively or resorption of grafted fat over time. Overcorrection can lead to several problems and is generally more difficult to resolve than undercorrection. Placement of excess adipose tissue in a particular area can lead to graft necrosis through failure of revascularisation. Fat migration may also be related to overcorrection of an area as pressure and insufficient blood supply force the graft into an undesirable site.

Contour irregularities may develop as a consequence of graft necrosis, surgical technique, or migration of grafted fat. Clumping can be corrected to some extent by scar massage.

Fat graft hypertrophy has been documented by several authors (Latoni, Marshall et al. 2000; Miller 2002), and is a problem particularly noted in patients with HIV-associated lipodystrophy. 
Damage to underlying structures such as nerves, muscles, glands and blood vessels is rare although can result from this technique. In particular, intra-arterial injection is a potentially devastating complication. Reports of unilateral blindness following treatment of glabellar frown lines have been documented as a result of intravascular emboli (Niechajev and Sevcuk 1994). The use of a blunt cannula is recommended to avoid these complications. Donor site contour irregularities can result from overly aggressive harvesting in a small area. Another problem relating to patients with HIV lipodystrophy is insufficient subcutaneous fat reserves in some patients secondary to generalized lipoatrophy. In patients with HIV lipodystrophy, fat is often harvested from areas of dystrophic fat (e.g. the dorso-cervical fat pad) which is extremely fibrous and often requires multiple aspirations.

Variable degrees of oedema can result from the procedure depending on the amount and location of grafted fat. Oedema is usually evident for 2 weeks after the procedure; however, may be prolonged and troubling to the patient.

Bleeding complications and bruising may also occur but are usually associated with the use of sharp needles for fat graft placement.

Although rare, infections can occur wherever the skin envelope is violated. The most common source of infection is the oral mucosa.

\subsubsection{Evidence for the use of autologus fat in HIV lipodystrophy}

Quantification of surgical outcome in HIV-infected patients undergoing autologous fat transfer for facial lipoatrophy is lacking, and studies are limited by small numbers, subjective assessment and lack of long-term data. In one study, 38 patients received autologous fat injections using the Coleman technique for HIV facial lipoatrophy (Serra-Renom and Fontdevila 2004). The cosmetic result was evaluated at six months, 
after which 12 patients required a new injection of fat to improve symmetry and resorption. At one year, the cosmetic result was rated as good to excellent by the patient, the clinic nurse and the surgeon. Less resorption was reported in cases where the fat was not centrifuged and the authors suggest that this may be due to decreased trauma to the fat. In another study, the outcome of the Coleman technique was evaluated in $33 \mathrm{HIV}$-infected patients by clinical examination, biochemical data, patient satisfaction and standardized photographs at baseline and one year following lipostructure (Burnouf, Buffet et al. 2005). Improvement was found in 12 patients by 3 independent evaluators at 12 months and $93 \%$ of patients were satisfied with the results. The authors also report that quantity of fat injected and low serum triglyceride level before surgery were significantly associated with improvement of facial lipoatrophy. Similar outcome measures were evaluated in two further studies to demonstrate the efficacy of lipostructure in HIV-infected patients using the Coleman technique for facial lipoatrophy. Levan et al (2002) reported $93 \%$ patient satisfaction and 'acceptable', 'good' or 'very good' results in 13 out of 14 patients evaluated by a 5-member jury 6 months following the procedure. Caye et al (2003) evaluated 29 HIVinfected patients treated using the Coleman technique and reports durability of fat grafts at 6 months based on serial photography. The results were deemed good in $72.4 \%$, acceptable in $13.8 \%$ and poor in $13.8 \%$. Davison et al (2007) have utilized lipoaspirate in patients undergoing suction-assisted liposuction for buffalo hump deformities to correct associated facial wasting. In these cases, the fat grafts were not centrifuged to avoid aerosolization of the virus. The authors report graft take of approximately 40 to 50 percent based on subjective assessment, and postulate that fat harvested from dystrophic sites possesses different biochemical properties from nonhypertrophic fat and may lead to more sustained results in these patients. However, Talmor et al feel that the quality of fat rendered from dystrophic sites makes it 
unsuitable for transfer due to its extremely fibrous nature (Talmor, Hoffman et al. 2002). The potential for fat graft hypertrophy when utilized from dystrophic sites should also be considered. Since HIV lipodystrophy is a systemic, metabolic disease, the effect of transferring dystrophic fat can be unpredictable. In one study of 41 patients who received autologous fat injections for HIV-associated lipoatrophy, four patients developed disfiguring facial lipohypertrophy at the injection site (Guaraldi, De Fazio et al. 2005). The fat source was the dorso-cervical fat pad in three of these patients. The pathogenic mechanism for this is unknown. The authors hypothesize that the mechanism may be related to adipocyte receptors and mitochondrial toxicity: receptor expression could be transferred from the harvest site and remain sensitive to lipohypertrophy determinants. Alternatively, lipohypertrophy of the cheeks may be the result of expansion of brown fat that is transferred with the intervention. Histological examination of hypertrophied fat graft to the nasogenian area was carried out following surgical resection in one patient. Fibroadipose tissue and dystrophic adipocytes of various sizes were demonstrated. The authors postulate that a nonadaptive response could be partly related to dysfunction of adipocyte receptor expression and a mutated DNA toxicity-related mechanism.

Despite the reported success of fat transfer, there is a lack of objective evidence; measurement to date has been subjective and based on patient and surgeon satisfaction. Although patient satisfaction is a key factor in determining outcome in aesthetic surgery, a more objective measure of fat transfer is required. Measurement of facial volume using three-dimensional imaging is an ideal modality and the technique shall be described further in the following chapter. 


\subsubsection{Dermis-fat graft}

The use of free fat grafts with overlying dermis for patients with soft-tissue defects was advocated by Peer in 1956. The results of dermafat graft transfer to the malar area in patients with HIV-associated lipodystrophy have been variable. Dermafat grafts were transferred from the abdominal wall to malar pockets through a trans-oral approach technique in five patients with antiretroviral-induced lipodystrophy of the face. (Stauch, Baum et al. 2004) Patients were overcorrected to twice the expected final volume and they reached final volume ( $50 \%$ of initial augmentation) in three to five months. The aesthetic results were maintained during the follow-up period of fourteen to thirty months. However, another report of the use of a dermis-fat graft in a patient with HIVassociated lipodystrophy is slightly less favourable (Wechselberger, Sarcletti et al. 2001). In this case, dermis-fat grafts were harvested from the buttocks to augment the nasolabial and temporal regions with overcorrection of the defect in a female patient with facial lipoatrophy. The patient was unhappy with the overcorrection at four weeks post-op and underwent removal of the bulky tissue, during which the parotid duct was lacerated on the right side. Despite an acceptable aesthetic result at 17 months, the authors remain skeptical about the technique.

\subsubsection{Rhytidectomy}

Rhytidectomy can ameliorate the disfigurement due to loss of facial volume by tightening the skin envelope although long-term follow-up results in patients with HIVassociated facial lipoatrophy are lacking.

\subsubsection{Temporary soft tissue fillers}

Filler substances play an important role in the treatment of the aging face and the correction of soft tissue defects. A significant body of evidence supports the use of fillers in the management of HIV lipodystrophy. Fillers can be classified as temporary, 
semi-permanent or permanent. Temporary fillers which have been utilized to treat HIVassociated facial lipoatrophy are discussed as follows. In general, recurrence of symptoms with the use of temporary fillers may either be treated by repeat injections of the filler or an alternative product, depending on patient and or practitioner preference.

1.3.4.1. Collagen fillers (Bovine: Zyderm ${ }^{\circledR}$ and Zyblast ${ }^{\circledR}$; Human: CosmoDerm ${ }^{\circledR}$ and CosmoPlast $($ )

Collagen has been used for over 25 years for cosmetic purposes. Large volumes of collagen may be needed to restore facial volume in patients with severe HIV-associated lipoatrophy, so this option is probably best for people with mild to moderate facial lipoatrophy.

More recently, several collagen-derived fillers have emerged (including Dermalogen ${ }$, Fascian ${ }^{\circledR}$, Isolagen ${ }^{\circledR}$, Autologen $®$ and AlloDerm $\left.®\right)$. To date, there have been few studies investigating the use of these products in the treatment of patients with HIVassociated lipodystrophy. In addition, information on long-term effects and duration of correction are limited.

\subsubsection{Calcium hydroxylapatite (Radiance ${ }^{\circledR}$, Radiesse $\left.{ }^{\circledR}\right)$}

Calcium hydroxylapatite consists of microspheres of calcium hydroxyapatite in a carboxymethylcellulose gel carrier. When injected into dermis, collagen forms around the calcium hydroxylapatite, providing long-term, natural-looking fullness. Eviatar et al have evaluated the safety and efficacy of Radiesse ${ }^{\circledR}$ in 100 patients with HIVassociated facial lipoatrophy. Eighty-five percent of patients received touch up injections at one month following initial treatment. The study demonstrated that all patients had improved aesthetic outcomes at six months follow-up as scored on a facial global aesthetic improvement rating scale by a panel of plastic surgeons; more than $90 \%$ of patients were scored as Very Much Improved or Much Improved. Adverse 
events were minor and included transient injection site reactions. There were no reports of granulomas or migration of the product (Eviatar, Silvers et al. 2005).

\subsubsection{Hyaluronic acid (Restylane ${ }^{\circledR}$, Perlane ${ }^{\circledR}$, Hylaform $\left.{ }^{\circledR}\right)$}

Hyaluronic acid is a naturally occurring polysaccharide component of the intercellular matrix. The use of hyaluronic acid has been evaluated for soft tissue augmentation of HIV-associated facial lipodystrophy in five patients (Gooderham and Solish 2005).

Each patient received approximately $5-6 \mathrm{mls}$ in total of hyaluronic acid in the malar area via intradermal injection. The technique was found to provide a good cosmetic result for at least six months, with high patient satisfaction and no adverse events. Another study of seven patients with HIV-associated facial lipodystrophy treated with hyaluronic acid revealed high patient satisfaction with regards to cosmetic improvement, and no side effects of treatment (Ritt, Hillebrand-Haverkort et al. 2001).

\subsubsection{Poly-L-lactic acid (Newfill ${ }^{\circledR}$, Sculptra $\left.{ }^{\circledR}\right)$}

Poly-L-lactic acid was approved by the FDA in August 2004 for treatment of HIV associated facial atrophy. Poly-L-lactic acid (PLLA) (NewFill ${ }^{\circ}$, Sculptra ${ }^{\circledR}$ in the US) is a biocompatible, bioabsorbable synthetic polymer from the alpha-hydroxy-acid family. Injectable PLLA is composed of crystalline, irregularly sized microparticles of poly-L-lactic acid. The product is supplied as a sterile, freeze-dried preparation which also contains carboxymethylcellulose as a suspending agent to maintain even distribution of poly-L-lactic acid particles and nonpyrogenic mannitol that enhances the lyophilization process. The mechanism by which injectable poly-L-lactic acid produces the observed volume change is related to a foreign body giant cell reaction that occurs several weeks to months after injection. This is followed by a progressive increase in collagen deposition as the bioactive material is degraded and undergoes resorption. This neocollagenesis is thought to account for the gradual and volumizing effect of 
PLLA. Poly-L-lactic acid polymers degrade through nonenzymatic hydrolysis to lactic acid monomers, which are in turn metabolised into carbon dioxide or incorporated into glucose (Borelli, Kunte et al. 2005). Poly-L-lactic acid differs from other dermal fillers in that it leads to gradual volumetric expansion over time, and the objective of treatment is to enhance volume-deficient areas rather than correct discrete facial deficiencies such as lines and folds.

There is a growing body of literature on the use of PLLA in the correction of facial lipoatrophy. To date, there have been four principal clinical studies involving PLLA for the correction of HIV-associated facial lipoatrophy: VEGA, Chelsea and Westminister, APEX002 and Blue Pacific.

The VEGA study evaluated the safety and efficacy of Poly-L-lactic acid injections (Newfill@) in 50 patients with HIV-associated lipoatrophy by means of an open-label, single-arm pilot study over 96 weeks (Valantin, Aubron-Olivier et al. 2003). Patients received four sets of injections given every 2 weeks: at day 0 and at weeks 2,4 , and 6 . One vial of PLLA per cheek per treatment session was utilised and reconstituted with 3-4 $\mathrm{ml}$ of water for injection. Clinical endpoints included: change in total cutaneous thickness from baseline; improvement in quality of life; change in viral load, CD4 count and blood chemistry parameters; and incidence of adverse events. All patients experienced increases in skin thickness in the treatment area. Statistically significant increases above baseline values of mean skin thickness were noted at all time points (weeks $8,24,48,72,96$ ) during the study. Based on results of the visual analogue scale, there was a significant improvement in quality of life compared with baseline values. There were no serious adverse events, although in $44 \%$ of patients, palpable but non-visible subcutaneous nodules developed. 
Similar results were found by Moyle et al, who conducted a 24-week open-label, study in $30 \mathrm{HIV}$ patients with facial lipoatrophy, designed to compare immediate treatment with delayed treatment of PLLA (Moyle, Lysakova et al. 2004). Patients randomized to the immediate treatment group received PLLA injections on day 1 and at weeks 2 and 4. Patients in the delayed group received injections at weeks 12,14 and 16 . Clinical endpoints included ultrasound measurement of skin thickness, assessment of anxiety and depression, visual analogue scale of patient satisfaction, and changes in laboratory values. Ultrasonographic assessment revealed significant changes from baseline in the areas treated with $\mathrm{Newfill} \circledast$ in all patients. A mean increase in dermal thickness of 4 to $5 \mathrm{~mm}$ within 12 weeks of treatment was reported with effects persisting at least 18 weeks beyond the last injection. Statistically significant improvements in anxiety and depression and patient-assessed visual analogue scores were also demonstrated. APEX002 and Blue Pacific were open-label, single centre studies aiming to assess safety and efficacy of PLLA over 12 months. During the APEX002 trial, 99 patients were given 1-6 treatment sessions, 4-6 weeks apart. Treatment efficacy was measured by patient and investigator satisfaction and digital photography. Patients and physicians rated the degree of lipoatrophy on a scale of $1-5(1=$ mild, $5=$ severe $)$ and patient satisfaction was also rated on a scale of $1-5$ ( $1=$ dissatisfied, $5=$ very satisfied $)$. Mean lipoatrophy at baseline was 3.58 in the cheeks, decreasing to 1.51 prior to final treatment, then 0.87 and 0.88 and 6 and 12 months respectively. At the final treatment the average satisfaction rating was 4.71. In the Blue Pacific study, 97 patients received 1-6 treatment sessions three weeks apart (Mest and Humble 2006). Clinical endpoints included measurement of total cutaneous thickness by skin callipers, assessment of serial photographs and patient satisfaction. At the final treatment visit, mean skin thickness increased by $65.1 \%$ from baseline. At 6 and 12 months following treatment, 
skin thickness had increased by $68.8 \%$ and $73 \%$ respectively. High levels of patient and investigator satisfaction were also reported. The main drawback of this study relates to the use of skin capilers as an outcome measure of cutaneous thickness, which is highly operator dependent. Also no precise surface landmarks for measurements are identified. Based on clinical impression and patient observation, one author reports stability of PLA until 30 months in 2131 patients treated for facial rejuvenation, although these results have not been substantiated using objective measures in a clinical trial (Vleggaar 2006).

Adverse events from treatment can be divided into injection-related and device-related. Injection related side effects were similar across the studies and included pain, swelling, bruising, erythema, haematoma, and inflammation which resolved spontaneously within $24-48$ hours in most cases. The most important device-related adverse event was the formation of small, subcutaneous papules (SPs) which can be classified as visible or non-visible, temporary or permanent or early or late. Histologically, the nodule consists of fluid droplets or microparticles surrounded by a normal foreign body reaction. It is claimed that they may arise as a result of technical error, uneven distribution of PLLA in suspension, or uneven dispersal in the injected areas(Lam, Azizzadeh et al. 2006). In the VEGA study, $44 \%$ of patients developed SPs on average 7 months after initial injection (range $0.3-25$ months); however, in $27 \%$ of those patients, the SPs had disappeared at week 96 . Thirty-one percent of the patients in the Chelsea and Westminster study developed SPs, whereas $6 \%$ of patients in the APEX002 study and $12 \%$ of patients in the Blue Pacific study developed this complication. None of the study protocols describe evaluation or treatment of papules. Lam et al suggest that early nodules (that appear within several weeks of treatment) should be treated by needle fragmentation and late nodules (that appear within several 
months of treatment) by intralesional steroid injection. Failure to respond to these treatments may require local excision of the papule.

Granuloma formation represents another device-related adverse event with the reported incidence ranging from $0.2-12 \%$. They typically arise throughout the treated area rather than at discrete sites several months to years following injection. Local excision fails to address the problem as the borders of a granuloma are seldom well defined. Steroid therapy with or without immune-modulating medications is the mainstay of intervention (Vleggaar 2006).

Some authors now recommend that the incidence of SPs may be reduced by increasing the volume of dilution, reconstituting a minimum of 12 hours prior to use, subcutaneous injection, and increasing the treatment interval to 4-6 weeks (Borelli, Kunte et al. 2005; Lam, Azizzadeh et al. 2006).

Several published studies have utilised ultrasound to measure cutaneous thickness; however, prior to commencement of trial, there were no published studies to evaluate outcome using three-dimensional imaging. In the VEGA study, patients were assessed using an unspecified 'quality of life' scale. In the Chelsea and Westminister study, patients were assessed using the Hospital Anxiety and Depression Scale (Zigmond, Snaith et al. 1983) and a self-devised visual analogue scale to measure change in body shape. Neither of the studies used a validated measure of body image assessment, such as the Derriford Appearance Scale.(Carr, Moss et al. 2005)

\subsubsection{Semi-permanent and permanent fillers}

The use of semi-permanent and permanent fillers may present an attractive treatment option for both cosmetic and reconstructive patients who desire a long-term aesthetic outcome. However, the decreased immunity inherent to the HIV-positive surgical patient represents a significant concern. The self evident concern for any surgeon is the 
ongoing risk of peri-implant infection in a patient cohort who may become infection prone and subject to a high incidence of dental caries and skin disease.

\subsubsection{Silicone oil}

Silicone oil has been used for facial lipoatrophy but its safety and effectiveness for cosmetic purposes remain controversial. A ten-year follow-up of silicone injections for facial atrophy revealed that silicone oil is safe and effective when injected in very small amounts, dispersed throughout the tissue planes (Rees, Ashley et al. 1973).

Disadvantages of using silicone include risks of infection, granulomas, migration of silicone droplets and inability to remove the product. There have been reports on the use of liquid injectable silicone in patients with HIV-associated facial lipoatrophy including a case report of one patient (Orentreich and Leone 2004) and larger study of 77 patients (Jones, Carruthers et al. 2004). In both papers, 1000-cs liquid injectable silicone was administered using the microdroplet serial puncture technique. Both papers conclude that silicone oil is a safe and effective treatment for HIV-associated facial lipoatrophy. Facial contours were restored and significant patient satisfaction was achieved with no adverse events noted. However, longer-term safety and efficacy in HIV patients remains to be proven.

\subsubsection{Polyalkylamide gel (Bio-Alcamid) ${ }^{\circledR}$}

Bio-Alcamid $\AA$ is a relatively new hydrogel polymer that is used for cosmetic and reconstructive purposes. It is an alkylic resin containing $96 \%$ pyrogen-free water and $4 \%$ alkylimide-amide groups. The product is considered to be a permanent filler due to the stability of its chemical properties and resistance to hydrolysis (Ramires, Miccoli et al. 2005). Once injected, Bio-alcamid becomes enclosed within a thin $(0.02 \mathrm{~mm})$ collagen capsule (Protopapa, Sito et al. 2003). The process commences within a few days of injection and is completed by 6 weeks. Electron microscopy analysis of skin 
biopsy specimens three months after Bio-alcamid implantation revealed fibroblasts arranged around the injected material, with no signs of inflammation (Formigli, Zecchi et al. 2004). Bio-alcamid is thus considered to be an endoprosthesis (an intermediate between an injectable filler and a prosthesis) due to the encapsulation process which isolates the injected material from surrounding host tissues. This is reported to contribute to the safety of the product and prevent migration of injected material (Formigli, Zecchi et al. 2004). A number of safety studies have shown Bio-alcamid to be non-toxic, non-allergenic, non-reactive, biocompatible and non-biodegradable (Pacini, Ruggiero et al. 2003). Studies by Lotti et al have suggested a weak inflammatory reaction of cellular tissue surrounding the implant, with diminishing numbers of CD68 macrophages after 4 months in the upper perivascular dermis. Immune-histochemical studies have also demonstrated a lack of fibroblast induction. Several advantages of Bio-alcamid have been proposed. Once injected and settled, the implant has the consistency of the normal tissues and a total, permanent correction can be achieved in just one or two sessions. The procedure requires no surgical incisions, and is minimally invasive. When compared to other fillers, polyalkylimide gel can be used in large quantities, and if lipoatrophy continues or the patient is overcorrected, it can be partially or totally removed to obtain a more natural or symmetrical look (Pacini, Ruggiero et al. 2003).

Studies providing clinical evidence for the use of Bio-alcamid in cosmetic and reconstructive practice have been published. The largest multicentre study of 2000 otherwise healthy patients reported excellent cosmetic results with only 12 complications.(Pacini, Ruggiero et al. 2002) These results have also been replicated in several case series of patients with HIV-associated facial lipoatrophy. One Italian study has reported stability, tolerance and safety of Bio-Alcamid® with permanent, 
satisfactory improvement in appearance for patients with lipodystrophy (Protopapa, Sito et al. 2003). In this study, $90 \%$ of the 73 patients required a second injection of Bio-Alcamid $\AA$ (four weeks after the first injection) to improve implant appearance. The aesthetic results were deemed excellent by both physicians and patients at follow-up of three years. Favourable clinical experience with Bio-alcamid was reported by Lahiri et al for a variety of reconstructive purposes including 15 patients with HIV facial lipoatrophy, although one patient developed infection with Staphylococcus aureus (Lahiri, Waters et al. 2007). High patient and physician satisfaction were reported following treatment of thirteen patients with HIV facial lipoatrophy in another study, with no major complications (Ramon, Lucian et al. 2007). However, all of these reports are based on subjective outcomes only. In addition to photographic assessment, some studies utilising quality of life (QoL) measures as treatment outcomes for HIV lipoatrophy have been published. In one study of 31 patients randomised to immediate or delayed injections to correct facial lipodystrophy, 2 QoL questionnaires (Medical Outcomes Study HIV Health Survey and the modified Dermatology Quality of Life Survey) and the Hospital Anxiety and Depression scale (HADS) were completed (Loufty, Raboud et al. 2007). Compared with the delayed group, patients in the immediate therapy group had significantly lower physician-related lipoatrophy scores, improved QoL and lower anxiety scores. Significant improvements in patient anxiety and depression were observed from baseline to week 48. Three QoL measures were assessed in another study of 17 patients receiving treatment for HIV facial lipodystrophy with Bio-alcamid.(Karim, deLInt et al. 2008) Relevant parts of the Short Form 36-item health survey, the Medical Outcomes Study HIV Health Survey, and the Centre for epidemiological Studies were used to assess outcome. QoL improved significantly from baseline over 48 weeks for mental health and social functioning and depression was significantly correlated with the extent to which lipoatrophy had 
disappeared at week 48. Although early published studies demonstrated the safety and efficacy of Bio-alcamid $\AA$, the clinical follow-up period was limited. Longer-term complications relating to the use of the product including infection, migration and capsule formation are beginning to emerge (Karim, Hage et al. 2006; Jones, Carruthers et al. 2007) and shall be monitored in this study.

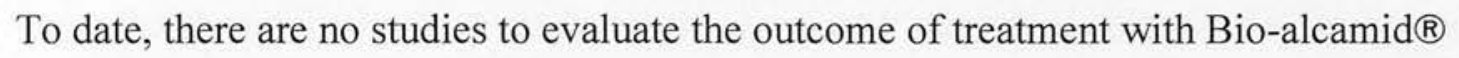
using the Derriford Appearance Scale or 3-D imaging techniques.

\subsubsection{Polymethylmethacraylate (PMMA; Artecoll ${ }^{\circledR}$, Artefill@)}

Artecoll ${ }^{\circledR}$ consists of polymethylmethacrylate suspended in bovine collagen, which is degraded and replaced by autologous collagen approximately three months after injection (Alster and West 2000). Side effects of Artecoll® include erythema, swelling, moderate pain lasting an average of 48 hours, and mild pruritis at the implantation site for several weeks. It has been suggested that correct placement of the implant between the dermis and subcutaneous fat rather than within the dermis may avoid some of these problems (McClelland, Egbert et al. 1997). The use of polymethylmethacrylate for HAART-associated lipoatrophy has been reported from Brazil, but no structured studies have been performed (Serra, Oyafuso et al. 2004).

\subsubsection{Implants}

\subsubsection{Silicone implants}

The use of submalar silicone implants has been evaluated in patients with HIVassociated lipodystrophy. In one study, three patients with facial lipoatrophy underwent reconstruction with submalar silicone implants via an intra-oral approach (Talmor, Hoffman et al. 2002). Two patients required additional soft-tissue augmentation; one patient received collagen augmentation of the nasolabial fold at an unspecified postoperative interval, and the other patient received collagen and autologous fat 
augmentation after progression of atrophy at fifteen months. No infection or extrusion was encountered, although one patient required implant repositioning on one side. At 15 months follow-up, both patients and surgeons were satisfied with the results. The advantages of this technique are that silicone implants provide a durable surgical reconstruction, the procedure can be performed on an outpatient basis under local anaesthetic, and the implant can be easily removed if necessary compared to an injected material. The main disadvantages include infection and displacement of the implant.

\subsubsection{Polytetraflouroethylene Facial Implants}

The use of e-PTFE soft tissue augmentation in plastic and reconstructive surgery has been favourable, with a high degree of patient and surgeon satisfaction (Levine and Berman 1995). However, larger series with longer follow-ups are necessary to assess safety and efficacy, and studies specifically in HIV lipodystrophy patients are lacking.

\subsubsection{Flaps}

With the advent of microvascular surgery and free flap transfer, numerous flaps have been used to augment facial soft tissue deformities. One patient with lipodystrophy is reported to have undergone facial contour reconstruction using a double paddle dermisfat forearm free flap (Endo, Nakayama et al. 1994). Another option described for patients with lipodystrophy is the adipofascial free radial forearm flap (Koshy and Evans 1998). Since dermis is not incorporated, the flap is malleable, enabling it to be contoured to the shape of the defect. It is also reported to maintain bulk after five years follow-up in two patients.

A temporalis muscle rotation flap has also been described to augment the cheeks and upper lip in a patient with lipodystrophy (van der Wal and Mulder 1998). Potential disadvantages with free tissue transfer include multiple procedures, lengthy operation, flap failure, haematoma, and donor site defects. 


\subsection{Measures of psychological outcome}

\subsubsection{Psychological aspects of HIV lipodystrophy}

The psychological consequences of HIV lipodystrophy are well recognized amongst practitioners involved in the management of patients with HIV lipodystrophy. The stigmatizing effect of body changes may influence health-related quality of life (incorporating self-image, self-esteem, social interaction and sexual relations), and anxiety and depression (Colins, Wagner et al. 2000). Facial lipoatrophy, in particular appears to be associated with the most detrimental psychological effects. This is in keeping with other psychological studies evaluating facial disfigurement and is due to the unique importance of our facial appearance in terms of our 'identity'. The face is exposed and is the focus of communication as well as being central in others' perception of an individual (Tebble, Thomas et al. 2004). For some patients, the negative impact of lipodystrophy leads to non-compliance with antiretroviral therapy (Corless, KM et al. 2005). Although several studies have attempted to quantify psychological distress, these often rely on non-standardized methods. Reynolds et al conducted a qualitative study to capture the psychosocial dimensions of HIV lipodystrophy. They reported physical, psychological and social distress across sex, sexual orientation and geographic subgroup, but highlighted that the greatest fear for patients was unintentional disclosure of their HIV status. Whilst this study provides descriptive evidence for psychosocial impairment, it does not attempt to quantify these elements (Reynolds, Neidig et al. 2006). Goetzenich et al evaluated the correlation between body habitus changes and specific areas of health related quality of life (HRQL). They found that a diagnosis of lipodystrophy was correlated with decreased social contact, changes in daily performance, changes in sexual functioning and decreased self-esteem, although the study lacked a standardized HRQL measure 
(Goetzenich, Corzillius et al. 2000). A study by Falutz found that $70 \%$ of 82 patients with HIV lipodystrophy reported depressed mood, with facial lipoatrophy having the greatest impact on patients (Falutz 2003). However, a standardized measure of depression was not used; therefore, the criteria to diagnose mood alterations is uncertain. Although the body of published literature report negative psychological sequelae of lipodystrophy, one study has found no associated between lipodystrophy and HRQL or depression (Oette, Juretzko et al. 2002).

Plastic surgery is now the mainstay of treatment for established lipodystrophy as studies have shown that medical intervention is generally ineffective. Several authors have reported improvements in psychological wellbeing in patients undergoing treatment for HIV lipodystrophy using a variety of validated and non-validated scales. Studies evaluating the outcome of treatment with polylactic acid, such as the VEGA trial, have reported improved quality of life extending to 48 weeks post-treatment (Valantin, Aubron-Olivier et al. 2003). However, a non-standardized visual analogue scale was used and the particular elements assessed are not described. Some authors have incorporated validated tools (such as the Short Form 36-item health survey, the Medical Outcomes Study HIV Health Survey, the Centre for epidemiological Studies, and the Hospital Anxiety and Depression Scale) to assess psychological outcome following treatment with polyalkylimide gel and report significant improvement in quality of life, anxiety and depression extending to 48 weeks (Karim, Hage et al. 2006; Loufty, Raboud et al. 2007). However, the selected measures were developed and validated in the pre-HAART era, at a time when the lipodystrophy was not yet recognized and the clinical picture of HIV was different to the present day. Two further studies comparing autologous fat transfer, polylactic acid and polyalkylimide gel have found improved quality of life in all three treatment groups (Guaraldi, Orlando et al. 2005; Negredo, Higueras et al. 2006). Measures used in these studies included the 
Assessment of Body Change and Distress questionnaire (ABCD) and the Beck

Depression Inventory. Although, the $\mathrm{ABCD}$ was specifically designed to analyze the impact of HIV-related lipodystrophy, this questionnaire has no specific items referring to facial lipoatrophy. Therefore, none of the scales employed to assess quality of life address concern over physical appearance specifically relating to the face. Traditionally the outcomes of aesthetic surgery have relied on subjective measures such as patient or surgeon satisfaction. The need for more objective outcomes is becoming increasingly recognized. This requirement is particularly important when assessing the benefits and consequences of treatment for HIV lipodystrophy.

Price has developed a model of body image with 3 related components: body reality (the way our body really is), body ideal (our perception of how our body should look) and body presentation (how we present our body to others)(Price 1995). The balance of these components leading to normal body image is influenced by individual coping strategies and social support network. An altered body image occurs when the body no longer supports self-esteem, is dysfunctional and limits social activity. Price emphasizes that a change in appearance does not necessarily lead to a state of altered body image, but that the interpretation of change is the determining factor.

Previous research has suggested a number of factors may influence psychological outcome and body image perception in disfiguring conditions including age, sex, ethnic origin, socio-economic class, site of disfigurement and cause of the injury. Social influences vary depending on age (Janelli 1986), and women may be more sensitive than men to changes in body image (Anderson and Maksud 1994). It has been reported that patients in low socio-economic classes are more affected by their state of health. Partridge has also reported that the site of facial disfigurement is important; with disfigurements in the communication triangle (between the eyes and mouth) provoking more negative reactions than elsewhere in the face (Partridge 1993). Interestingly, this 
is the area mainly affected in patients suffering from HIV facial lipoatrophy. In terms of aetiology of injuries, accidents and assault have the greatest psychological impact. In patients with HIV associated lipoatrophy, the impact of HIV infection and other healthrelated concerns clearly may also influence body image assessment and general psychological wellbeing. In addition, a high proportion of HIV patients seeking treatment for HIV lipodystrophy are men who have sex with men (MSM). There is a paucity of research which utilizes standardized measures to compare psychological distress between HIV seropositive patients with and without lipodystrophy. In addition, there is little published evidence evaluating the relationship between sexual orientation and psychological morbidity in an MSM control group.

\subsection{Three-dimensional Imaging}

\subsubsection{Three-dimensional Imaging Techniques}

Methods to record facial dimensions are useful to study morphology, to develop treatment plans and to evaluate treatment outcomes. There are advantages and disadvantages of each of the registration methods which have evolved.

\subsubsection{Anthropometry}

Anthropometrical measurements may be used to study facial morphology and have been used extensively to collect normative data (Farkas and Posnick 1992) and to study craniofacial abnormalities such as cleft lip and palate in adolescent and adult populations (Farkas, Hajnis et al. 1993). Ghoddousi et al also demonstrated good levels of repeatability of the technique when compared with 3D stereophotogrammetry and 2D photography (Ghoddousi, Edler et al. 2007). The technique has some advantages including the ability to locate landmarks by palpation. However the analysis is limited to linear and angular measurements. The technique is highly operator dependent, timeconsuming and requires a great deal of subject co-operation. In addition, 
anthropometrical instruments may introduce deformation of the surface of the face and are unable to measure some structures such as the eyes.

\subsubsection{Facial casts}

Plaster casts obtained via impression may be used to obtain a 3-D model of the face. Applications of this technique include the creation of facial casts for burns patients and anthropometric analysis of the cleft infant face (Krimmel, Kluba et al. 2006). This method demands full co-operation of the patient during the session of impression and is usually performed under general anaesthesia in children. Inaccuracy due to deformation of soft tissues may occur during the procedure. Holberg et al have demonstrated marked differences between the cast and facial surfaces using a three-dimensional laser scanner (Holberg, Schwenzer et al. 2006).

\subsubsection{3-D Cephalometry}

3-D cephalometry is a technique of manually abstracting 3-D co-ordinate data from lateral and anteroposterior cephalometric films. The concept was introduced by Broadbent and first implemented by Singh and Savara (Singh and Savara 1966). The advantage of using radiographs is that the morphology of the underlying hard tissues can be registered (Ras, Habets et al. 1996). The disadvantages of this technique include radiation exposure, inaccuracy of landmark location and the time required to extract data. Computer programs can now extract three-dimensional co-ordinates from digital cephalogram images (Dean, Hans et al. 2000).

\subsubsection{Morphanalysis}

Morphoanalysis is a technique promoted by Rabey which utilizes photographs, radiographs and study casts of a patient to obtain 3-D information (Rabey 1971). It is claimed to be accurate and statistically valid; however, the required equipment is expensive and the process is time-consuming.

\subsubsection{3-D Facial Morphometry}


Ferrario et al have described a system for recording three-dimensional co-ordinates of facial landmarks (Ferrario, Sforza et al. 1994). The system consisted of two charged coupled device (CCD) cameras, real time hardware for the recognition of markers and software for the $3 \mathrm{D}$ reconstruction of the landmark coordinates. The process is timeconsuming, requires considerable patient cooperation, and landmark placement is restricted due to the size of the markers necessitating $2 \mathrm{~mm}$ separation between the landmarks. The technique also lacks a 3D facial image display since only the landmarks are recorded.

\subsubsection{Electromagnetic digitisation}

Electromagnetic three-dimensional digitisers can be used directly on facial soft tissues to supply the coordinates of facial landmarks (Ferrario, Sforza et al. 1997). A hand-held probe is used to locate facial landmarks and 3-D co-ordinates are produced. This method has the advantage that the landmarks are identified directly on the subjects' face rather than on a reconstruction of the face. Its main disadvantage is the compliance required which makes its use in infants impractical.

\subsubsection{3-D CT scanning}

The first commercial CT scanner appeared in 1972 and its' potential to generate 3D information was quickly recognized. In the 1980s, CT-assisted 3D imaging was introduced for use in maxillo-facial surgery (McCance, Moss et al. 1992). Since then, the applications and popularity of this technique have increased dramatically. CT scanning makes it possible to analyze the soft and hard tissues of the head. Computer software can reconstruct a 3D image from successive slices. The error of 3 dimensional CT data is considered to be minimal, although the accuracy of reconstructed $3 \mathrm{D}$ images 
depends on the thickness of the original slices (Ras, Habets et al. 1996). It is useful in diagnosis and treatment planning of patients with craniofacial abnormalities (Fisher, Lo et al. 1999). Stereolithographic techniques can be used to manufacture models for surgery but require experienced operators (Burke and Beard 1967). The technique may also be used in the production of various prostheses, including ear prostheses (Watson, Coward et al. 1993). However, there are several drawbacks of 3D CT scanning including: (1) patient exposure to a high dose of ionizing radiation; (2) limited resolution of facial soft tissues due to slice spacing; (3) the possibility of artifact form metal objects; (4) patient co-operation during long scanning periods. Sedation or anaesthesia may be required to obtain images of infants.

\subsubsection{MRI}

Three-dimensional images can also be reconstructed from magnetic resonance imaging (MRI) (Moss 2006). This is a useful technique for 3D imaging of soft tissues and may be used to estimate tissue depth in human faces with precision. (13) Its main advantage is that it does not involve a radiation dose. The drawbacks of this technique include: cost relative to other techniques; artifacts from metal objects; unable to differentiate between air and bone; and discomfort for patients who feel claustrophobic in an enclosed environment (Browne, Golding et al. 2001). Similar to CT, sedation or anaesthesia may be required to obtain images of infants.

\subsubsection{Moire topography and contour photography}

Both Moire topography and contour photography use grid projections during exposure, resulting in standardized contour lines on the face (Kawai, Natsume et al. 1990). A grid with known projections is projected onto the face and transforms into an image of contour lines on the face. The contour lines can then be used to calculate the depth of the image based on contour fringes and fringe intervals. These methods are better suited to use on smoothly contoured faces as difficulties are encountered if a surface 
has sharp features. Also a small change in head position can result in a large change in fringe pattern, thus accurate head positioning is critical (Rabey 1971).

\subsubsection{3-D Laser scanning}

Laser scanning techniques provide a less invasive method for capturing the face in three dimensions. These methods make use of the detection of a reflected laser beam to reconstruct 3-D images of the surface of objects. The earliest descriptions of this technique was in 1985 (Arridge, Moss et al.). A low-energy laser light beam is passed through a cylindrical lens so that a vertical line is projected onto the surface. A video camera at an angle to the light source records the image of this line and the camera output is digitized into a set of 3-D co-ordinates. The patient is rotated in a motorized chair in order to scan the whole face. The laser scanning system was subsequently used to describe changes in facial morphology following a variety of orthognathic procedures (McCance, Moss et al. 1992).

The accuracy of this system was evaluated by comparison of laser-scanned landmarks with direct anthropometric measurements. The most reliable measurements were in the nasal and circumoral regions, with a mean difference of less that $1.0 \mathrm{~mm}$ (Aung, Ngim et al. 1995). More recently, a study of 3-D laser-scanned images of a mannequin head demonstrated a mean error of the scanned image of $0.37 \mathrm{~mm}$. Kau et al analyzed 38 adults one week apart using a 3-D laser imaging system. The mean difference of superimposed images was $0.37 \mathrm{~mm}$ and $0.35 \mathrm{~mm}$ for males and females respectively, indicating reproducibility of the system within one week of initial records (Kau, Richmond et al. 2006). The 3-D laser system has the advantage of capturing an image in a simple and rapid manner, with the ability to immediately view the image at any preferred angle and position. The data can be easily retrieved and repeated scans can be performed without radiation hazards making it useful for long-term studies. Several 
models of the face scanned at different angles can be computed into a composite image to create a $360^{\circ} 3 \mathrm{D}$ image.

A major drawback of this technique is the time required to capture image, during which the patient must not change his or her facial expression. Improvements in technology have reduced the time to capture images from 10 seconds with the earlier systems to around 1 second (Da Silveira, Daw et al. 2003).

However, difficulties remain when capturing images of young children, as any movement during the scanning interval results in distortion of the image. Another drawback of this system is that patient's eyes are required to remain closed during capture for protection, although a system has been described which allows image capture with the eyes open (Bush and Antonyshyn 1996). A further disadvantage is the inability of the system to capture the soft tissue surface texture, which can result in difficulties of landmark identification.

The cost of this system also remains a drawback.

\subsubsection{3-D Ultrasonography}

Three-dimensional ultrasonography delivers a reflection picture which is transformed into digital information using a specific contact probe to generate a 3-D database (Hell 1995). This system produces 3-D co-ordinates of selected landmarks but does not create a 3-D image. The procedure requires a skilled operator as well as a co-operative patient. Also distortion of facial soft tissues with the probe may occur which alters their spatial positions.

\subsubsection{Stereophotogrammetry}

Photogrammetry is defined as 'the science or art of obtaining reliable measurements by means of photographs' (Hajeer MY 2002). Stereophotogrammetry utilizes 2 cameras, configured as a stereo-pair, to recover the $3 \mathrm{D}$ distance to features on the surface of the 
face by triangulation. It was first described in the medical literature in 1967 (Burke and Beard 1967). Contemporary digital stereophotogrammetry incorporates advances in computer science technology to convert simple photographs to 3-D images based on complex algorithms. Various systems applied to capture the face have been described. These include the Spatial Vision System (Bowskill, Baldock et al. 1997) stereophotogrammetry systems (Rasse, Forkert et al. 1991; Ras, Habets et al. 1996) and video-based stereophotogrammetry (Stevens 1997).

The $\mathrm{C} 3 \mathrm{D} \otimes$ imaging system was developed as a result of collaboration between Glasgow University Dental School and the Turning Institute. The system was based on the Active Stereo Probe which employed a new image-matching algorithm and consists of two stereo digital cameras and special textured illumination. It has been used to record 3D records of study models (Ayoub, Garrahy et al. 2003) and to study the facial soft tissue changes following orthognathic surgery (Hajeer, Mao et al. 2005). A drawback of this system is the requirement for the scene to be bathed in 'textured light' to capture the natural surface appearance of the patient's skin. This technique is termed 'active stereophotogrammetry'. A new system has been developed by Dimensional Imaging, a company branched from C3D Limited, which is able to extract 3-D models from stereo-pair images without restoring to bathing the scene in speckle illumination. This technique, termed 'passive stereophotogrammetry', is possible due to the development of high-resolution digital cameras which can accurately capture surface texture. 


\subsubsection{Facial volume measurement}

The assessment of facial morphology using 3-D imaging has often relied on the measurement of distances, angles, and curves between defined facial landmarks. Other techniques using statistical analysis are based on geometric morphometrics. This is a method where the coordinates of the landmarks are statistically analysed rather than interlandmark distances (O'Higgins and Jones 1998). Three-dimensional coordinates are defined in three planes; the $\mathrm{x}$ axis is the horizontal plane, the $\mathrm{y}$ axis the vertical plane and the $\mathrm{z}$ axis the anteroposterior plane.

Changes have been described in terms of 3D facial soft tissue landmark displacement (Berkowitz and Cuzzi 1977), 3-D interlandmark distances and angles (Ras, Habets et al. 1996), 3-D mesh diagram analysis (Ferrario, Sforza et al. 1998), colour-coded millimetric maps of changes on 3-D soft tissue models (McCance, Moss et al. 1992), and other 3-D shape analyses such as Euclidean distance matrix analysis (O'Grady and Antonyshyn 1999).

The development of software to measure facial volume is still under investigation by researchers but represents a key application of $3 \mathrm{D}$ imaging. The problem with all methods of facial volume assessment is that the area under measurement needs to be closed. The surface of the face does not have an inherent volume therefore a plane has to be constructed to form the posterior border of a face and close the volume. When examining pre and post-operative swelling, if the swelling is localised, the overlap of the two images may give a closed volume. However, if a border has to be constructed to close the volume between two models, the location of this plane could have a dramatic effect on the volume and render the analysis meaningless. 
A method of measuring the volume of a facial swelling has been described (Bowskill, Baldock et al. 1997) using a Spatial Visual System (SVS). The SVS acquires four video images (two stereo-pairs) and constructs a 3-D surface representation using stereomatching of spatial features using 'speckled' illumination. A simulated pre-operative and post-operative volume was imaged using a plaster head onto which a quantity of padding was added over the left cheek. Volumetric quantification was achieved by volume differencing and the mean error in repeated measurement of volume was found to be within $3.5 \%$. The authors advised that this was a 'best case' error since the images were of a cast and not a live subject. Another method of measuring facial swelling has been described (Yip, Smith et al. 2004) which evaluated the accuracy and reproducibility of the Fiore ${ }^{\top M}$ 3-D range camera by simulating facial swellings on a mannequin head and 20 human subjects. Image registration was carried out by adjusting the second image over the first image using the forehead as a control area. A colour-graded format was used to display regions of facial swelling, and volume was calculated after drawing a mask over the area. In a validation of this method the overall percentage difference for the volumetric comparisons ranged from $0.1 \%$ to $2.3 \%$. Validation of the C3D system for facial volume analysis was conducted in vitro using a mannequin head and in vivo with a male-subject head (Hajeer, Mao et al. 2005). Thirty facial silicone moulds were applied to various regions of the face and their volumes were calculated using three different algorithms (the 'tetrahedron formulation', the 'projection' method and the 'back-plane construction' method.) The least mean error, found with the tetrahedron formulation, was $0.071 \mathrm{~cm}^{3}$ in vitro and $0.314 \mathrm{~cm}^{3}$ in vivo.

A further method of measuring volume, as a measure of asymmetry, has been described (O'Grady and Antonyshyn 1999). In this method, a laser-scanned plaster model with and without a silicone cheek implant was used to simulate facial asymmetry. The 
volume was determined by superimposing and subtracting one facial half from a mirror image of the contralateral half. However, an error of about $16.2 \%$ was found. Facial volume analysis has also been applied to study facial growth (Ferrario, Sforza et al. 1998). This technique involved locating 3D landmarks on the face and connecting them in a series of triangles with a posterior plane. Only the approximate volume of the face is therefore quantified and the results cannot be compared to other techniques which measure the whole surface of the face. Kobayashi et al carried out volumetric changes in the lower face before and after orthognathic surgery by dividing the $3 \mathrm{D}$ model into eight sites, with six intersecting planes (Kobayashi, Ueda et al. 1990). The drawbacks of this study are that these mathematically constructed volumes are not clinically applicable, and the accuracy and reproducibility of the imaging technique were not evaluated.

Clinical studies to evaluate facial soft tissue volume change following treatment with fillers are lacking, particularly in patients with HIV lipodystrophy. Therefore, this study will provide objective analysis of facial changes secondary to injection of biological fillers and fat.

\subsubsection{DI3D Facial Camera}

\subsubsection{Description of DI3D Facial Camera system}

The DI3D Facial Camera is a precision 3D surface-image capture device which was used in this study to capture 3-D facial images. The system consists of two pods, each pod containing two 10 megapixel digital cameras, attached to a base and tripod stand. The cameras are assembled vertically (right top and bottom and left top and bottom) on either side of the base which measures $87 \mathrm{~cm}$ in width. The cameras are positioned at 
angles of 30 degrees. The system is attached to a laptop computer for image capture.

(Figure 1.1)

Figure 1.1. DI3D Facial Capture System

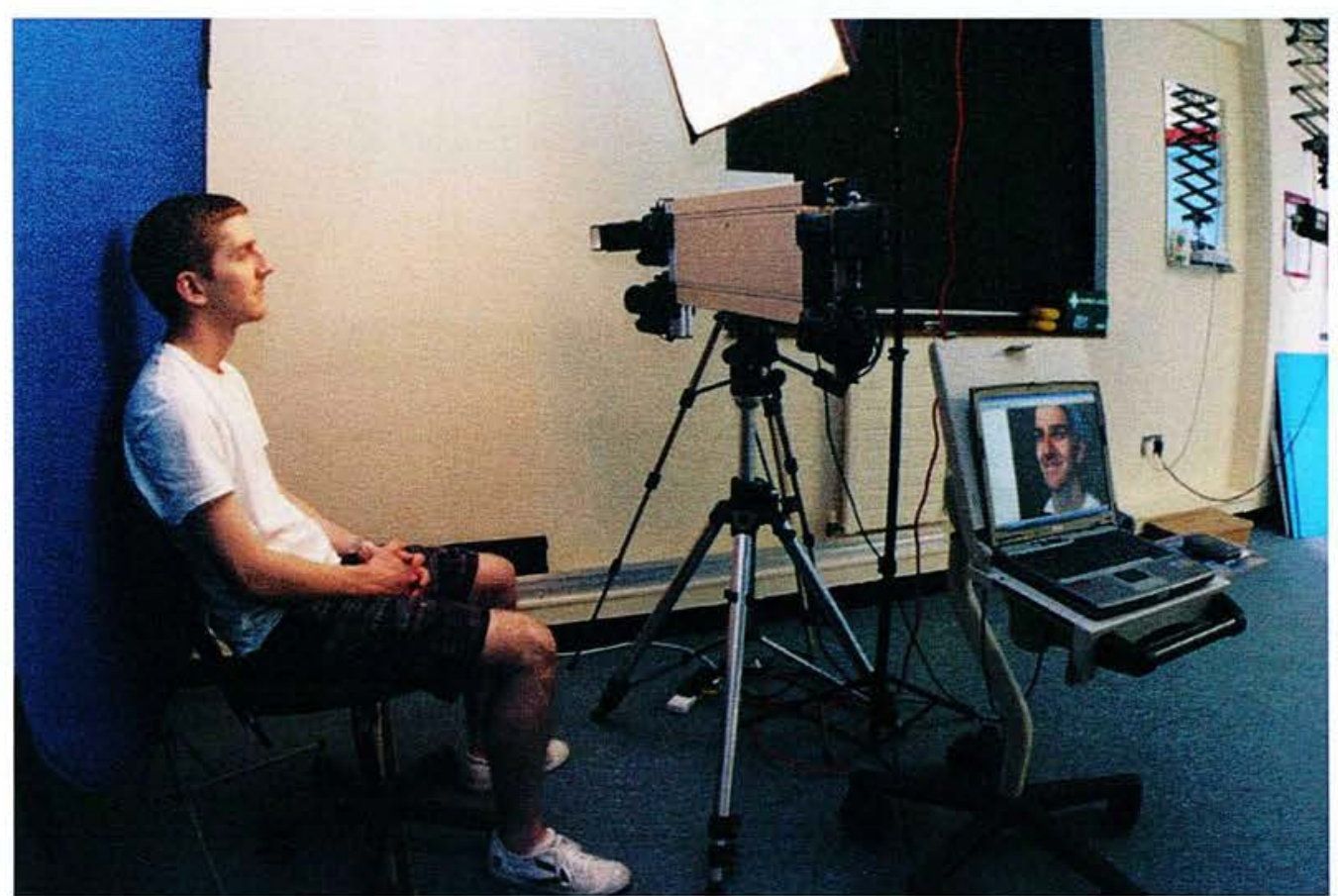

\subsubsection{Principles of Stereophotogrammetry}

The DI3D system uses a passive stereo photogrammetry technique using SLR (single lens reflex) digital cameras to produce high-resolution 3D surface images. Passive stereo photogrammetry utilizes the subject's skin pattern to triangulate the geometry from each surface point. There are several processes are required to produce the 3-D surface model.

\section{Capture}

The DI3D system captures two stereo-pairs of images using four cameras (Figure 1.2). 
Figure 1.2. Images captured from each pod.
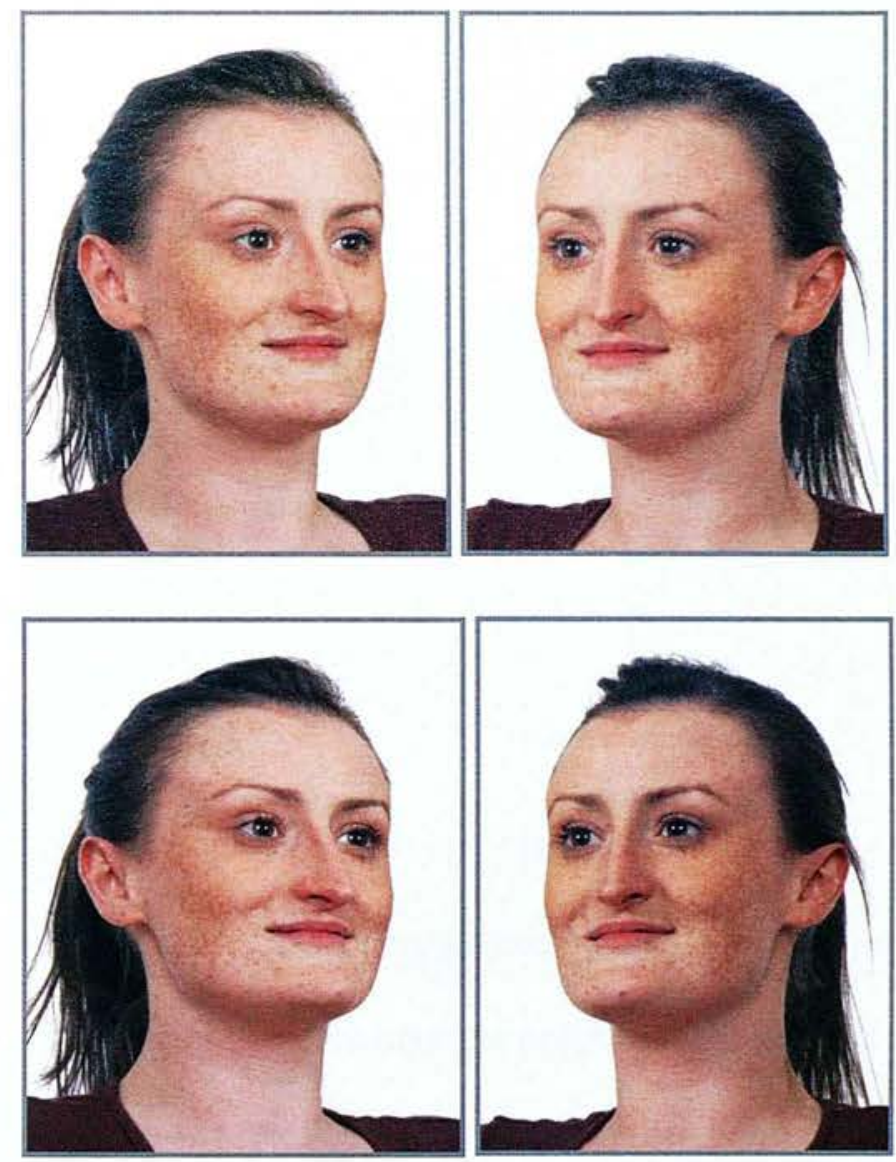

This increases the number of 3-D measurements that can be obtained to compute a 3-D facial surface model. With a synchronized trigger, the four images are captured simultaneously, which reduces the inaccuracy due to movement.

\section{Triangulation and stereo}

DI3D creates a depth map by decoding parallaxes from a stereo-pair of images. This is similar to human vision in that we perceive depth from slight parallaxes present between the views observed in each eye. Figure 1.3 illustrates this principal. 
Figure 1.3 Principle of stereophotogrammetry

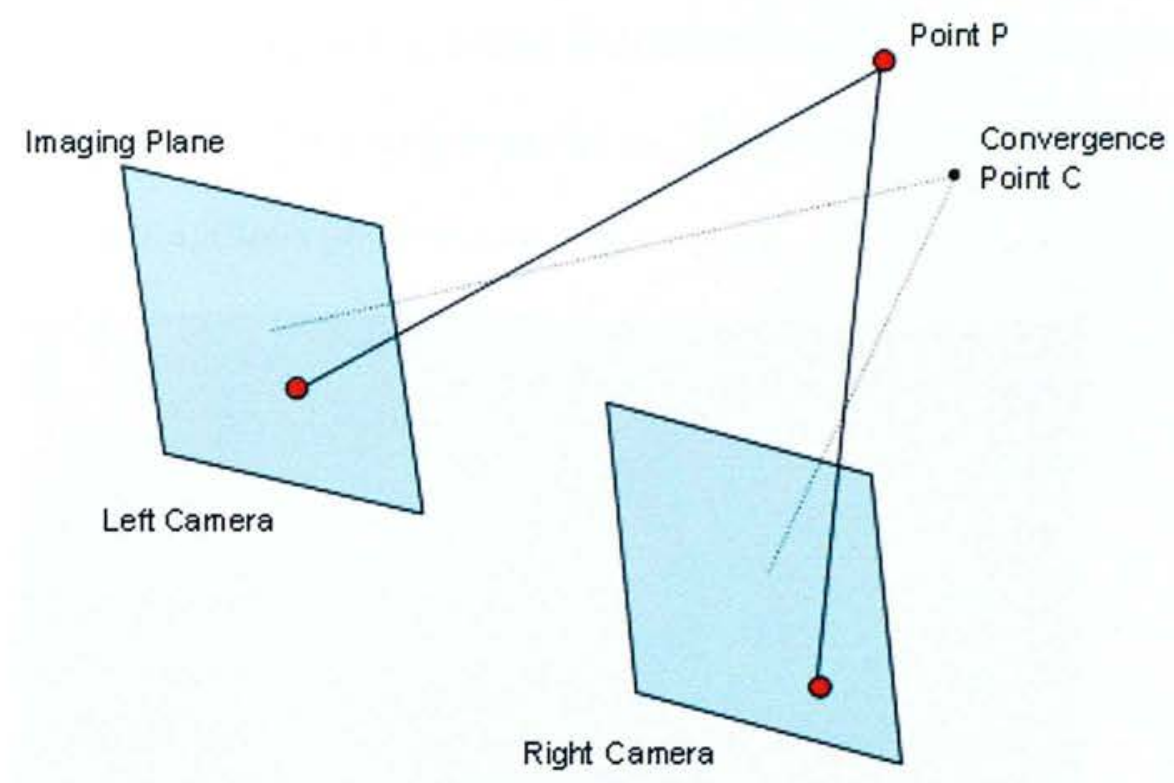

Point $\mathrm{P}$ in space will project onto the left and right cameras in slightly different

locations. The difference is termed parallax or disparity, and increases as point $\mathrm{P}$ moves further from the convergence point $\mathrm{C}$ of the camera stereo-pair. Similarly, the sign of disparity reverses depending on whether point $\mathrm{P}$ lies in front or behind point $\mathrm{C}$. The DI3D software is able to produce depth values based on the size and sign of the disparity values.

\section{Stereo matching}

The next stage in the process is to determine for each point imaged in the left camera, the corresponding point in the right camera. This process, termed stereo matching, produces an $(\mathrm{x}, \mathrm{y})$ disparity map. This maps the values of each pixel co-ordinate of the left image to the right image for each stereo-pair. A confidence map is also produced which gives an indication of the reliability of each matched value. The process of stereo matching also utilizes the geometry of the camera system deduced by calibration. A calibration target, comprising a series of black dots on a white background, of known dimensions and location is imaged in a series of different angles to the cameras. The geometric configuration the cameras can be determined by processing images of the 
target from each camera to find the central location of the dots. By computing disparities from stereo matching with calibration data (a process termed space intersection) a cloud point is produced. (Figure 1.4)

Figure 1.4. Cloud point images
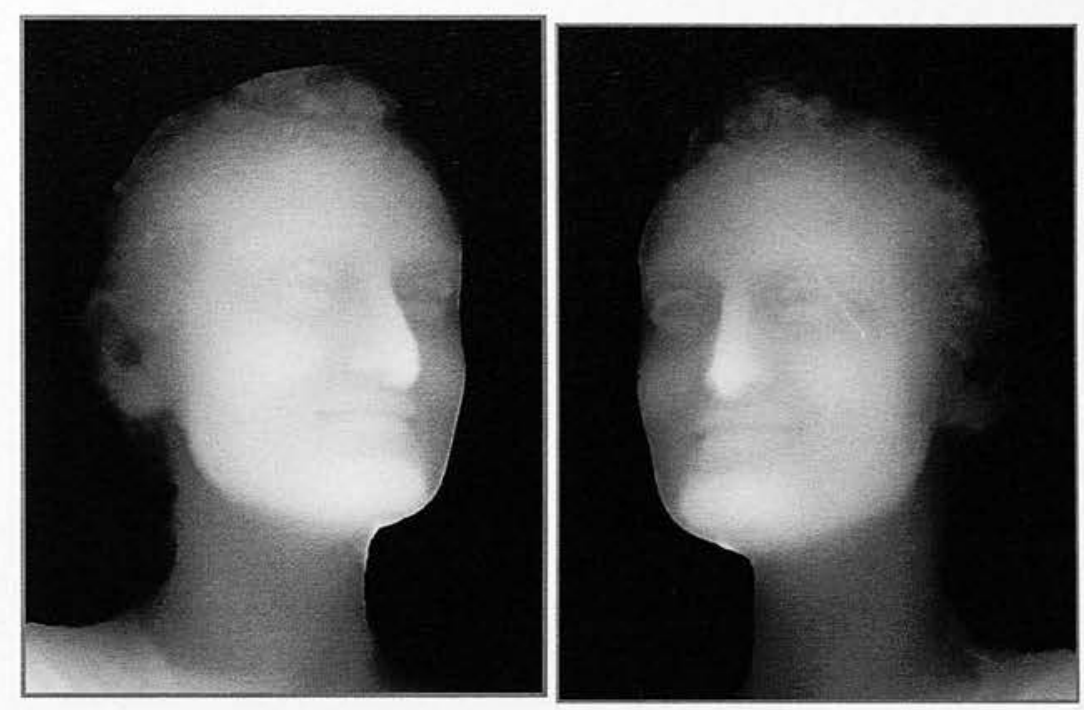

\section{Model construction and integration}

The point cloud (also called a range model) comprises only $2.5 \mathrm{D}$ information but can be easily warped to fit a triangulated mesh. The point clouds from each pod (each set of cameras) can be integrated to create the $3 \mathrm{D}$ image. During calibration, the orientation of each pod with respect to the calibration target is calculated. This process enables the point cloud captured from each pod to be transferred into the same coordinate frame. The point clouds are merged into a single triangulated polygon mesh using the marching cubes algorithm. The final 3-D model can be seen as a solid, shaded, or wireframe model (Figure1.5).

This method is non-invasive, captures the face in 50 milliseconds and does not expose the patient to harmful radiation. Therefore, this system was chosen as the most useful tool to capture facial images in a clinical trial. When the study was initiated, the DI3D system required development of volume software to analyze the change in measured outcomes following surgery. A software package was developed by the Dimensional 
Imaging engineers and tailored to the specific requirements of the study. However, validation studies to evaluate the accuracy and reliability of the system and software to measure facial volume was required and shall be described in the following chapter. 


\section{Chapter 2. Surgical technique and outcome following treatment for HIV facial lipoatrophy}

\subsection{Aim}

The aim of this study was to evaluate three different treatments for HIV facial lipoatrophy with regards to surgical technique, complications and patient satisfaction.

\subsection{Method}

\subsubsection{Design}

This was a prospective, follow-up study in a cohort of patients undergoing 3 treatments for HIV associated facial lipoatrophy: autologous fat transfer, Newfill and Bio-alcamid.

\subsubsection{Sample Size}

A retrospective review of all patients with HIV lipodystrophy treated within the Edinburgh unit prior to commencement of the trial was conducted to evaluate local demographic data and treatment outcomes. Thirty-two patients with HIV lipodystrophy were treated within the Edinburgh unit between March 2003 and March 2006 and identified using the hospital coding system. Thus, a sample size of $50 \mathrm{HIV}$ lipodystrophy patients was chosen to allow the study to be completed within a reasonable timescale whilst providing useful information for future studies.

With 50 patients followed up post-operatively, the standard deviation of a continuous outcome can be estimated with a $95 \%$ confidence of $\pm 20 \%$. There was likely to be some loss to follow-up, but by collecting data at several time points and analysing with repeated measures techniques, the study would provide sufficient data to obtain accurate estimates of post-operative outcome distributions. 


\subsubsection{Ethical Approval}

Full ethical approval was granted from the Multi Centre Research Ethics Committee for Scotland for the project to be carried out at multiple clinical sites within NHS Lothian and Greater Glasgow. In addition, Research and Development approval at each site was awarded.

This study also received funding from the following charities:

- Henry Smith Charity

- NHS Lothian Endownments

- William Rooney Charity

- Sick Kids Foundation, Edinburgh

\subsubsection{Recruitement}

Patients and physicians were made aware of the trial via the SHIVAG (Scottish HIV and Aids Group) website and annual meeting. Posters to advertise the trial were also placed in HIV clinics in Edinburgh and Glasgow. Patients seeking treatment for HIV lipodystrophy were referred by their HIV physician to the Plastic Surgery Unit in either Edinburgh or Glasgow. Referred patients were appointed to the out-patient clinic of the lead consultant involved in the trial from each unit. At the out-patient clinic, patients were assessed by the Plastic Surgery Consultant and lead researcher. A full medical history and clinical examination was carried out, incorporating the patient and physician assessment of lipodystrophy devised by Carr et al (Appendix 3). Patients were recruited to the trial if the eligibility criteria were satisfied. A patient information sheet was provided and informed consent was obtained. The patients' HIV physician and general practitioner were informed by letter, only if patient consent was obtained. Inclusion Criteria:

1 Patients over 18 years of age. 
2 Male or female.

3 HIV positive.

4 Patient and physician agreed body changes attributable to lipodystrophy and amenable to surgical treatment.

5 Undetectable viral load and CD4 count $>200$ cells $/ \mathrm{mm}^{3}$

Exclusion Criteria:

1 Pregnancy.

2 Inflammation/ infection of skin at site of treatment.

3 Concurrent illness/ medical problems precluding general anaesthetic.

4 Concurrent treatment with anti-coagulants.

5 Any known psychiatric disorder

\subsubsection{Data Collection}

Demographic data was collected including the patients' age and ethnic status. Data relating to the patients' HIV status was recorded including; date of diagnosis, mode of HIV acquisition, current medication and treatment history, viral load/ CD4 count. Information on the patients' past medical history, drug history and relevant social history were also collected.

The patient and physician assessment of lipodystrophy (Carr, Law et al. 2003) was used to collect information on clinical features of lipodystrophy (Appendix 1). Each patient completed a lipodystrophy-specific questionnaire and underwent a lipodystrophyspecific examination by the lead researcher. Both assessments recorded independently any lipoatrophy or fat accumulation in each of the face, neck, dorsocervical spine, arms, breasts, abdomen, buttocks, and legs, as well as the presence of any lipomata. The degree of lipoatrophy or fat accumulation at each region was rated as absent $=0$, mild $=1$ (noticeable on close inspection), moderate $=2$ (readily noticeable by patient 
and physician) or severe $=3$ (readily noticeable to a casual observer) by both the patient and surgeon.

Anthropometric measurements obtained for each patient included height, weight and skin-fold thickness. The height and weight were used to calculate the Body Mass Index $\left(\mathrm{BMI}=\right.$ weight $(\mathrm{kg}) /$ height $(\mathrm{m})^{2}$. Measurements of skin-fold thickness were obtained from five sites (triceps, biceps, abdomen, thigh and medial calf) and recorded in accordance with the International Standards for Anthropometric Assessment by the lead researcher using the Accu-Meaure Fitness 3000 body fat caliper.

\subsubsection{Treatment of HIV patients with facial lipoatrophy}

Three treatment options were available for patients presenting with facial lipoatrophy: autologous fat injections, Newfill or Bio-alcamid. During the clinical examination, potential donor sites for autologous fat transfer were assessed. Patients with sufficient subcutaneous fat were offered treatment with autologous fat injections. Patients with insufficient fat resources to facilitate autologous fat transfer, received treatment with either Newfill or Bio-alcamid. Patient preference was taken into account when choosing a treatment option.

\subsubsection{Autologous fat transfer}

Prior to theatre, patients underwent pre-assessment including medical history, examination, blood tests (FBC, U\&E, LFT, clotting, G\&S), ECG, and anaesthetic review. Lipoatrophic areas of the face and potential sites for fat harvesting were marked by the Consultant Plastic Surgeon, and informed consent for the procedure was obtained.

Under general anaesthetic, a solution containing $1 \mathrm{~L}$ saline, $40 \mathrm{mg}$ marcaine and $1 \mathrm{mg}$ adrenaline, was infiltrated to the subcutaneous fat of the periumbilical region, and/or areas of fat accumulation requiring liposuction. 
Fat was harvested using a Coleman cannula and $10 \mathrm{ml}$ syringe via small stab incisions

(Figure 2.1), then centrifuged for 3 minutes at low speed using the Coleman centrifuge machine.

Figure 2.1 Fat harvest from the periumbilical region

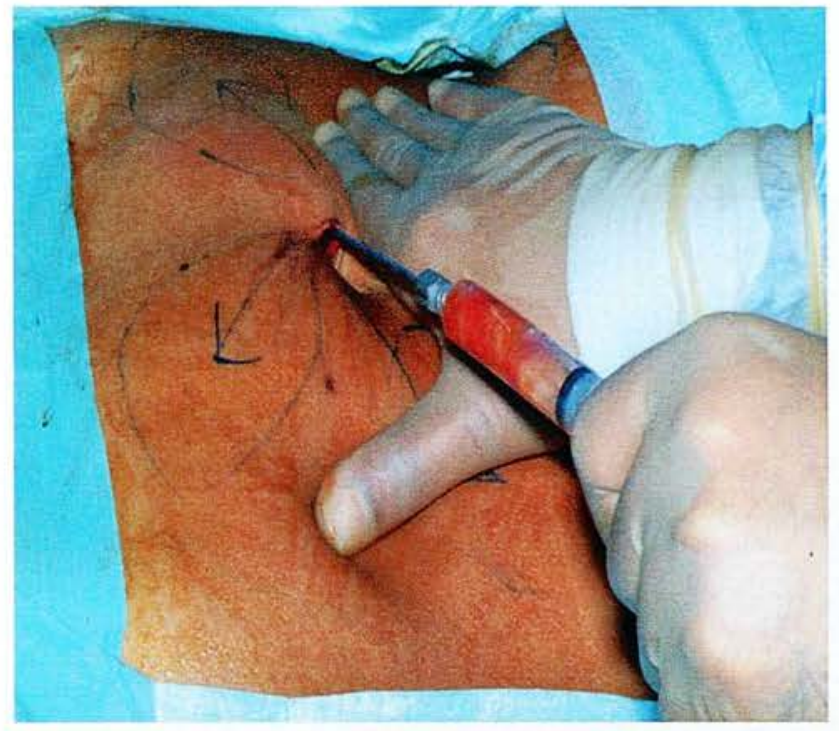

After centrifugation, the aspirate was divided into 3 layers (Figure 2.2): the top layer, consisting of free oil from ruptured fat cells, and the bottom layer, consisting of tumescent fluid and blood, were decanted. The middle layer, consisting of fat cells for grafting, was transferred to $1 \mathrm{ml}$ syringes for injection.

Figure 2.2. Centrifuged fat

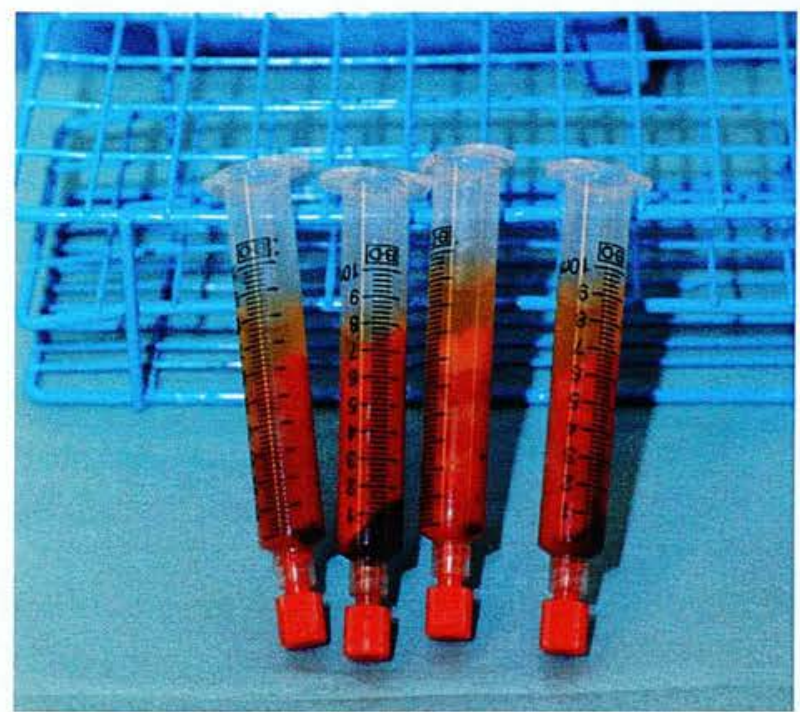


Fat was injected, via small stab incisions (at the nasolabial area, lateral zygoma and lateral eyebrow) to affected lipoatrophic areas using a blunt $2 \mathrm{~mm}$ Coleman cannula

(Figure 2.3). Fat was injected using a tunnelling technique. Multiple threads of $3 \mathrm{~mm}$ diameter fat were deposited in a retrograde fashion to multiple subcutaneous planes.

Figure 2.3. Fat injection technique

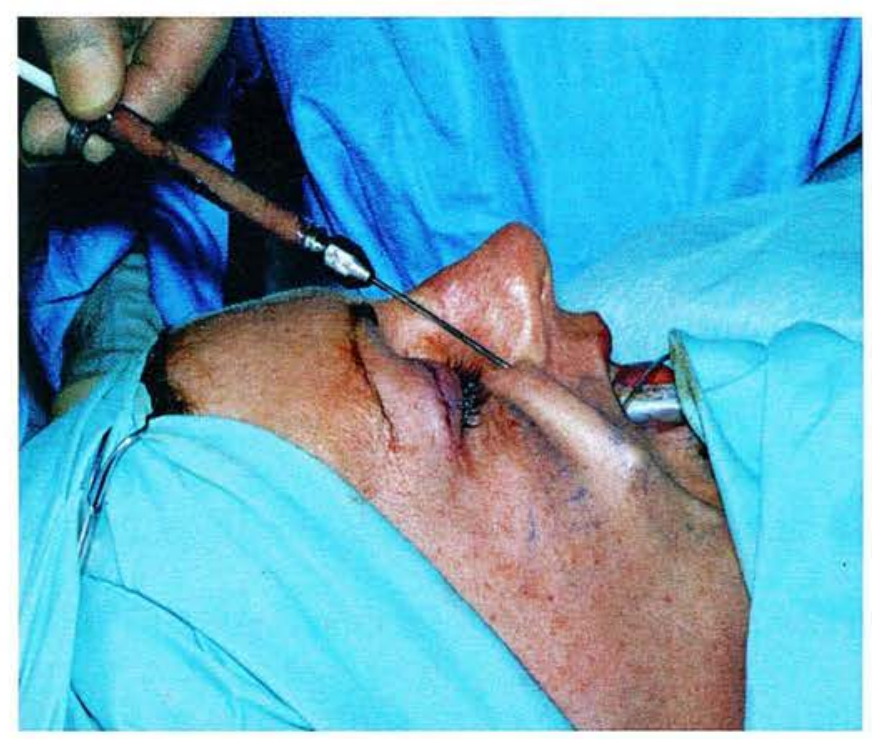

Some patients with areas of fat accumulation (such as the dorsocervical fat pad, anterior neck, gynaecomastia) had liposuction carried out during the same procedure. Occasionally, areas of fat accumulation were used as the donor site for fat transfer if subcutaneous fat from the abdomen was deemed insufficient. In these cases, harvested fat was aspirated using the method described to avoid excess trauma to the fat from mechanical aspiration.

Steri-strips were applied to all stab incisions and areas of liposuction were dressed with swabs and mefix tape.

Patients were observed in hospital overnight then reviewed in the out-patient clinic 2 weeks post-operatively to check the donor and recipient sites. 


\subsubsection{Newfill}

Newfill injections were carried out by a trained nurse specialist in the out-patient clinic. At the initial consultation, the patient was asked to identify the lipoatrophic areas of the face causing concern. The product is supplied as a sterile freeze-dried preparation in a clear glass vial. At each treatment session, one vial of Newfill per cheek was reconstituted with $3 \mathrm{mls}$ sterile water and $2 \mathrm{mls} 1 \%$ lignocaine into $1 \mathrm{ml}$ syringes. Reconstitution was carried out 2 hours prior to injection to ensure complete hydration, as per the manufacturer's recommendations. The product was then agitated immediately prior to use until a uniform translucent suspension was obtained.

The patients' face was prepared using sterile water, and the injection session was conducted using aseptic technique. Injection was carried out using a $26 \mathrm{G}$ sterile needle to the deep dermis of affected areas. The needle was introduced, bevel up, at an angle of 30-40 degrees until the desired skin depth was reached. For lipoatrophy affecting the malar area, a tunnelling technique for injection was used. The needle was advanced in the appropriate dermal plane, and the product was deposited as the needle was withdrawn. The volume of Newfill per injection was $0.1-0.2 \mathrm{~mL}$ and up to 20 injections were required per cheek. The depot injection technique was used for the temple area. Multiple small boluses of approximately $0.05 \mathrm{~mL}$ were injected to the temporal fascia and the area was massaged following each injection.

After the injection session, the treatment area was massaged to evenly distribute the product. Patients were advised to massage the treatment area daily for several days after the injection session to promote a natural-looking correction. Patients were advised about potential side-effects of treatment and given contact details to report problems.

Treatments were carried out at intervals of one month. At each session, the patient was re-evaluated to determine which areas required further correction. Up to 7 treatment 
sessions were required by some patients depending on the severity of facial lipoatrophy.

\subsubsection{Bio-alcamid}

Initially Bio-alcamid injections were carried out under general anaesthetic. Thus, patients required pre-operative assessment including medical history, examination, blood tests (FBC, U\&E, LFT, clotting, G\&S), ECG and anaesthetic review. In some patients, Bio-alcamid injections were performed using a regional nerve block. The areas of the patient's face requiring augmentation were delineated using a permanent marker pen pre-operatively.

The procedure was carried out in the operating theatre under aseptic technique.

Betadine solution was used to clean the patient's face and surgical drapes were applied. The head of the operating table was raised to 50 degrees to create an upright position. Antibiotic prophylaxis with augmentin was administered intraoperatively for patients having the procedure performed under general anaesthetic; or given as a 3-day postoperative course for patients having the procedure under local anaesthetic.

The product is supplied in pre-filled $3 \mathrm{ml}$ syringes and an $18 \mathrm{G}$ needle was used for injection (Figure 2.4).

Figure 2.4. Bio-alcamid syringes

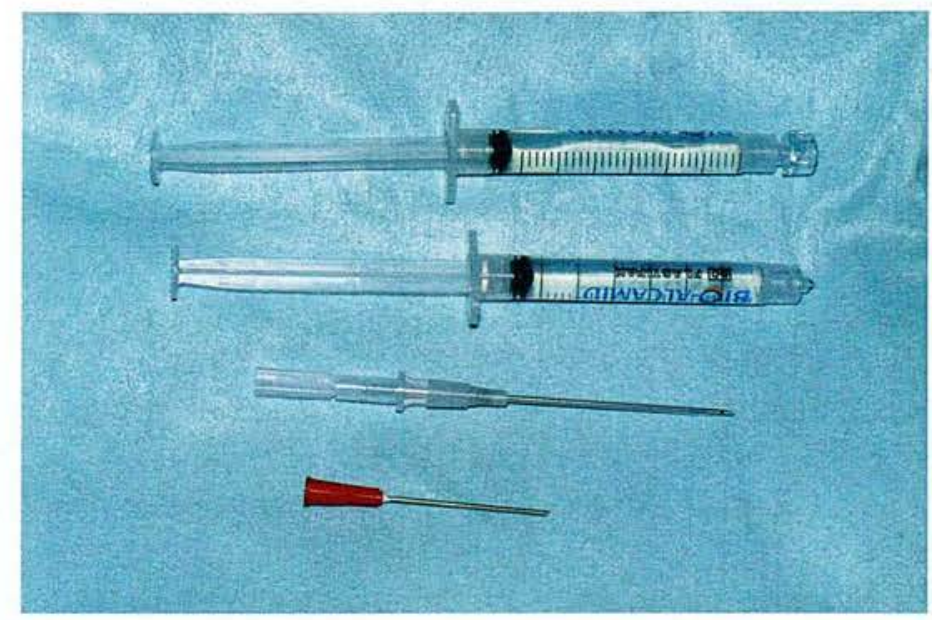


A hollow, beveled needle was used to pierce the skin then exchanged for a blunt needle. Injection sites for the cheek were the nasolabial fold and lateral zygoma at the pre-auricular region (Figure 2.5). For the temple region, the lateral brow or preauricular injection sites were used. Prior to deposition of the material, the blunt needle was used to create a pocket by fanning in the hypodermis of affected areas.

Figure 2.5. Bio-alcamid injection technique

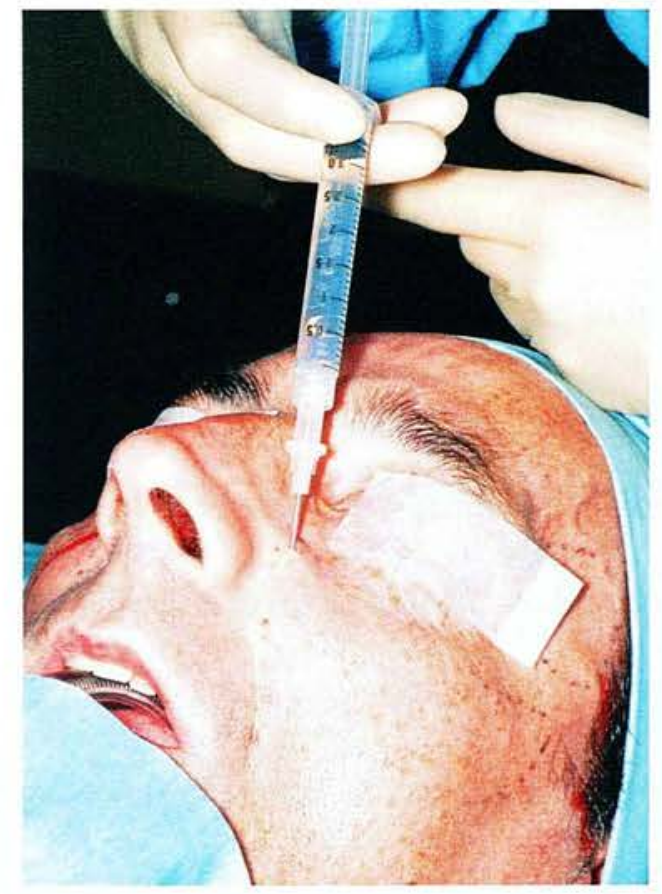

The product was injected until total correction of the deficit was obtained. Histoacryl glue was used to seal the injection sites. Patients were discharged from hospital on the day of the procedure and warned to avoid applying pressure or UV light to the treated area.

\subsubsection{Clinical Follow-up}

All patients were invited for clinical review at 2 months, 6 months and 12 months following treatment. This was undertaken either by the lead researcher or by lead consultant involved in the trail. Patients were asked about their satisfaction with the results of treatment, and about any complications following treatment. A subjective 
opinion of the patient was sought regarding recurrence of fat resorption or fat accumulation in treated areas and whether the results of treatment were maintained.

Patients were examined for asymmetry or irregularity in treated areas and also assessed for complications such as bruising, swelling, infection, nodules/ granulomas, migration, capsular contraction, and fistulae.

\subsubsection{Patient Satisfaction}

A series of visual analogue scales were designed for the study to assess patient satisfaction with treatment. Four questions were included (Appendix 2), intended to assess satisfaction with overall treatment, appearance improvement and patient expectations. Patients were asked to mark along a horizontal line with word descriptors at each end the point which they felt represented their current perception. The numbers one and ten were also placed at each end of the scale to facilitate the measurement of responses in an ordinal fashion. Scores were determined either by measuring in millimetres from the left hand end of the line to the point that the patient marked or recording the numerical value given by some patients on the scale.

\subsubsection{Statistical analysis}

Patient demographics, HIV-related factors and clinical features were summarised by treatment group (Autologous Fat Transfer, NewFill or Bio-alcamid) for patients undergoing treatment for facial lipoatrophy, and compared using analysis of variance (ANOVA) for continuous measures and Fishers exact test for categorical variables. Clinical features were summarised by gender. A descriptive summary of complications and their management was collated. Summary statistics were presented for the number of Newfill treatment sessions and displayed in a histogram. 
Patient satisfaction scores were subdivided according to treatment group and summary statistics were also measured.

\subsection{Results}

\subsubsection{Baseline characteristics of treatment groups}

Forty-eight patients with HIV lipodystrophy underwent treatment for facial

lipoatrophy. Three patients decided against treatment and one patient was excluded due to deteriorating HIV control and subsequent medical problems. 12 patients underwent Autologous Fat Transfer (AFT), 20 were treated with NewFill, and 16 with Bioalcamid.

The groups were well matched with regards to baseline age and HIV history (Table 2.1). The majority of patients were male. Only one (6\%) of the Bio-alcamid patients was female; a greater proportion of NewFill (20\%) and AFT (33\%) patients were female.

Similarly, the majority of patients were MSM in the Newfill and Bio-alcamid groups compared to $50 \%$ in the AFT group (reflecting the greater proportion of females in this group). Average BMI and skin fold thickness measurements were greater in the AFT group. Baseline mean clinical grading of facial lipoatrophy was greater for patient versus surgeon assessment for all groups and was significantly higher in the Bioalcamid group. Lipoatrophy only was present in $65 \%$ and $50 \%$ of patients treated with Newfill and Bio-alcamid respectively. However, $92 \%$ of patients in the AFT group demonstrated a mixed morphological pattern. 
Table 2.1. Demographics of fat, Newfill and Bio-alcamid treatment groups

$\begin{array}{lccc}\text { AFT } & \text { Newfill } & \text { Bio-alcamid } \\ (\mathrm{n}=12) & (\mathrm{n}=20) & \text { P value }\end{array}$

\begin{tabular}{|c|c|c|c|c|}
\hline \multicolumn{5}{|c|}{ Gender } \\
\hline $\begin{array}{l}\text { N (\%) Male } \\
\text { N (\%) Female }\end{array}$ & $\begin{array}{l}8(67) \\
4(33)\end{array}$ & $\begin{array}{l}16(80) \\
4(20)\end{array}$ & $\begin{array}{l}15 \\
(94) \\
1(6)\end{array}$ & 0.177 \\
\hline \multicolumn{5}{|l|}{ Unit } \\
\hline $\begin{array}{l}\text { N Edinburgh } \\
\text { N Glasgow }\end{array}$ & $\begin{array}{l}11 \\
1\end{array}$ & $\begin{array}{l}11 \\
9\end{array}$ & $\begin{array}{l}15 \\
1\end{array}$ & 0.012 \\
\hline \multicolumn{5}{|l|}{ Age (years) } \\
\hline Mean +- SD & $\begin{array}{l}43.4 \\
+-8.1\end{array}$ & $\begin{array}{l}45.5+- \\
11.4\end{array}$ & $\begin{array}{l}45.3 \\
+-4.9\end{array}$ & 0.78 \\
\hline \multicolumn{5}{|l|}{ Risk Factors } \\
\hline $\begin{array}{l}\text { N (\%) MSM } \\
\text { N (\%) IVDA } \\
\text { N (\%) Heterosexual } \\
\text { N (\%) Other } \\
\text { N (\%) Unknown }\end{array}$ & $\begin{array}{l}6(50) \\
2(17) \\
3(25) \\
0 \\
1(8)\end{array}$ & $\begin{array}{l}14(70) \\
2(10) \\
1(5) \\
1(5) \\
2(10)\end{array}$ & $\begin{array}{l}12 \\
(75) \\
1(6) \\
2(13) \\
0 \\
1(6)\end{array}$ & 0.758 \\
\hline \multicolumn{5}{|l|}{ HIV infection (years) } \\
\hline Mean +- SD & $\begin{array}{l}11.4 \\
+-5.2\end{array}$ & $\begin{array}{l}10.7+- \\
6.4\end{array}$ & $\begin{array}{l}12.2 \\
+-3.9\end{array}$ & 0.72 \\
\hline \multicolumn{5}{|l|}{ HAART duration (years) } \\
\hline Mean +- SD & $\begin{array}{l}9+- \\
3.8\end{array}$ & $\begin{array}{l}7.1+- \\
4.6\end{array}$ & $\begin{array}{l}9.2+- \\
3.5\end{array}$ & 0.28 \\
\hline \multicolumn{5}{|l|}{ CD4 count $\left(\right.$ cell $\left./ \mathrm{mm}^{3}\right)$} \\
\hline Mean +- SD & $\begin{array}{l}595+- \\
287\end{array}$ & $\begin{array}{l}551+- \\
239\end{array}$ & $\begin{array}{l}548+- \\
287\end{array}$ & 0.91 \\
\hline \multicolumn{5}{|l|}{ HIV RHA $<50$ copies/mL } \\
\hline $\mathrm{N}(\%)$ & $\begin{array}{l}12 \\
(100)\end{array}$ & $19(95)$ & $\begin{array}{l}16 \\
(100)\end{array}$ & 1.000 \\
\hline \multicolumn{5}{|l|}{$\mathrm{BMI}\left(\mathrm{kg} / \mathrm{m}^{2}\right)$} \\
\hline Mean +- SD & $\begin{array}{l}23.5 \\
+-2.2\end{array}$ & $\begin{array}{l}20.6+- \\
2.9\end{array}$ & $\begin{array}{l}21.4 \\
+-3.2\end{array}$ & 0.08 \\
\hline \multicolumn{5}{|c|}{ Skin fold thickness (mean of 5 areas, mm) } \\
\hline Mean +- SD & $\begin{array}{l}8.3^{+-} \\
3.3\end{array}$ & $\begin{array}{l}5.3+ \\
2.3\end{array}$ & $\begin{array}{l}5.1+- \\
2.7 \\
\end{array}$ & 0.008 \\
\hline \multicolumn{5}{|l|}{ Clinical grade } \\
\hline $\begin{array}{l}\text { Mean, Patient } \\
\text { Mean, Surgeon }\end{array}$ & $\begin{array}{l}2.1 \\
1.7\end{array}$ & $\begin{array}{l}2.1 \\
1.6\end{array}$ & $\begin{array}{l}2.7 \\
2.1\end{array}$ & $\begin{array}{l}0.016 \\
0.06\end{array}$ \\
\hline \multicolumn{5}{|l|}{ Clinical pattern } \\
\hline $\begin{array}{l}\text { N (\%) Lipoatrophy only } \\
\text { N (\%) Lipohyertrophy only } \\
\text { N (\%) Mixed morphology }\end{array}$ & $\begin{array}{l}1(8) \\
0 \\
11 \\
(92)\end{array}$ & $\begin{array}{l}13(65) \\
0 \\
7(35)\end{array}$ & $\begin{array}{l}8(50) \\
0 \\
8(50)\end{array}$ & 0.005 \\
\hline
\end{tabular}


Table 2.2 summarizes the morphological pattern of HIV-associated lipodystrophy in patients referred for treatment. The pattern varied between male and female patients: $87 \%$ of females presented with a mixed morphology whereas male patients demonstrated either lipoatrophy alone (45\%) or mixed morphology $(48 \%)$.

Table 2.2 Clinical patterns of HIV-associated lipodystrophy in patients referred for treatment.

\begin{tabular}{|c|c|c|c|}
\hline Pattern & & Male & Female \\
\hline \multirow[b]{2}{*}{$\begin{array}{l}\text { Lipoatrophy } \\
\text { only }\end{array}$} & N (\%) & $\begin{array}{c}19 \\
(45)\end{array}$ & $2(13)$ \\
\hline & $\begin{array}{l}\text { Facial atrophy } \\
\text { Peripheral } \\
\text { atrophy } \\
\text { Buttock wasting }\end{array}$ & $\begin{array}{c}19 \\
8 \\
4\end{array}$ & $\begin{array}{l}2 \\
2 \\
0\end{array}$ \\
\hline \multirow[b]{2}{*}{$\begin{array}{l}\text { Lipohypertrophy } \\
\text { only }\end{array}$} & N (\%) & $3(7)$ & 0 \\
\hline & $\begin{array}{l}\text { Gynaecomastia } \\
\text { DCFP } \\
\text { Anterior neck }\end{array}$ & $\begin{array}{l}1 \\
1 \\
1\end{array}$ & $\begin{array}{l}0 \\
0 \\
0\end{array}$ \\
\hline \multirow[b]{2}{*}{$\begin{array}{l}\text { Mixed } \\
\text { morphology }\end{array}$} & N (\%) & $\begin{array}{c}20 \\
(48) \\
\end{array}$ & $13(87)$ \\
\hline & $\begin{array}{l}\text { Facial atrophy } \\
\text { Abdomen } \\
\text { Peripheral } \\
\text { atrophy } \\
\text { Buttock atrophy } \\
\text { Gynaecomastia } \\
\text { DCFP } \\
\text { Anterior neck } \\
\text { Occipital fat pad } \\
\text { Parotid } \\
\text { hypertrophy } \\
\text { Breast } \\
\text { enlargement }\end{array}$ & $\begin{array}{c}19 \\
15 \\
11 \\
3 \\
6 \\
2 \\
4 \\
1 \\
1 \\
0\end{array}$ & $\begin{array}{c}8 \\
13 \\
11 \\
5 \\
0 \\
4 \\
1 \\
0 \\
0 \\
2\end{array}$ \\
\hline
\end{tabular}




\subsubsection{Complications of treatment}

\subsubsection{Bio-alcamid}

The majority of post-operative complications occurred with the use of Bio-alcamid and are summarised in Table 2.3.

Table 2.3. Summary of complications associated with the use of Bio-alcamid® in the Edinburgh Unit.

\begin{tabular}{|c|c|c|c|c|}
\hline Case & $\begin{array}{l}\text { Sex } \\
\text { Age }\end{array}$ & Complication & Onset & Treatment \\
\hline & & Infection & 2 months & Oral augmentin \\
\hline 1. & $\begin{array}{l}M \\
51\end{array}$ & $\begin{array}{l}\text { Capsule } \\
\text { formation/ } \\
\text { Inferior } \\
\text { migration }\end{array}$ & 12 months & $\begin{array}{l}\text { Expression of Bio-alcamid via } \\
\text { stab incision. }\end{array}$ \\
\hline \multirow[b]{2}{*}{2.} & \multirow{2}{*}{$\begin{array}{l}M \\
47\end{array}$} & Infection & 6 months & Oral augmentin \\
\hline & & $\begin{array}{l}\text { Inferior } \\
\text { migration }\end{array}$ & 16 months & $\begin{array}{l}\text { Removal Bio-alcamid \& } \\
\text { Coleman fat transfer. }\end{array}$ \\
\hline \multirow{2}{*}{3.} & \multirow{2}{*}{$\begin{array}{l}\text { M } \\
52\end{array}$} & Asymmetry & 1month & $\begin{array}{l}2 \text { attempts at removal via needle } \\
\text { puncture. }\end{array}$ \\
\hline & & $\begin{array}{l}\text { Intra-oral } \\
\text { extrusion }\end{array}$ & 12 months & $\begin{array}{l}\text { Surgical removal by stab } \\
\text { incisions/ curettage. }\end{array}$ \\
\hline \multirow{2}{*}{4.} & \multirow{2}{*}{$\begin{array}{l}\text { M } \\
45\end{array}$} & Asymmetry & 1 month & $\begin{array}{l}\text { Revision by further injection of } \\
\text { Bio-alcamid. }\end{array}$ \\
\hline & & $\begin{array}{l}\text { Chronic } \\
\text { inflammation/ } \\
\text { acute abscesses } \\
\text { at } 3 \text { sites. }\end{array}$ & 2 years & $\begin{array}{l}\text { Surgical removal by stab } \\
\text { incisions, curettage \& irrigation. } \\
\text { I.V. antibiotics \& long-term oral } \\
\text { antibiotics. }\end{array}$ \\
\hline \multirow[b]{2}{*}{5.} & \multirow[b]{2}{*}{$\begin{array}{l}M \\
41\end{array}$} & Asymmetry & 1 month & $\begin{array}{l}\text { Revision by aspiration \& further } \\
\text { injection of Bio-alcamid. }\end{array}$ \\
\hline & & Infection & $\begin{array}{l}1 \text { week } \\
\text { post- } \\
\text { revision }\end{array}$ & $\begin{array}{l}\text { I.V. flucloxicillin \& } \\
\text { benzlypenecillin }\end{array}$ \\
\hline \multirow[t]{2}{*}{6.} & \multirow[t]{2}{*}{$\begin{array}{l}\text { M } \\
39\end{array}$} & Asymmetry & 1 month & $\begin{array}{l}\text { Revision by } \\
\text { aspiration \& further injection of } \\
\text { Bio-alcamid }\end{array}$ \\
\hline & & $\begin{array}{l}\text { Excess capsule } \\
\text { formation }\end{array}$ & 3 months & None \\
\hline 7. & $\begin{array}{l}\mathrm{M} \\
59\end{array}$ & $\begin{array}{l}\text { Inferior } \\
\text { migration }\end{array}$ & 3 years & Removal of Bio-alcamid. \\
\hline
\end{tabular}

of the right cheek one week following a minor revision procedure. An ultrasound scan of the cheek demonstrated soft tissue swelling but no collection of pus. The most 
serious complications relating to infection were seen in a patient who developed chronic swelling of the left temple fourteen months post-operatively. Viscous fluid was present surrounding the capsule suggesting low-grade infection, although pus was subsequently aspirated from this region and both cheeks. Two months later, the patient presented with an acute abscess of the right cheek requiring incision and drainage. Microbiological assessment isolated clostridium perfringens and the patient was commenced on a prolonged course of oral metronidazole. At follow-up assessment, pus discharging from a sinus tract at the right cheek was discovered (Figure 2.6).

Figure 2.6. Sinus of right cheek

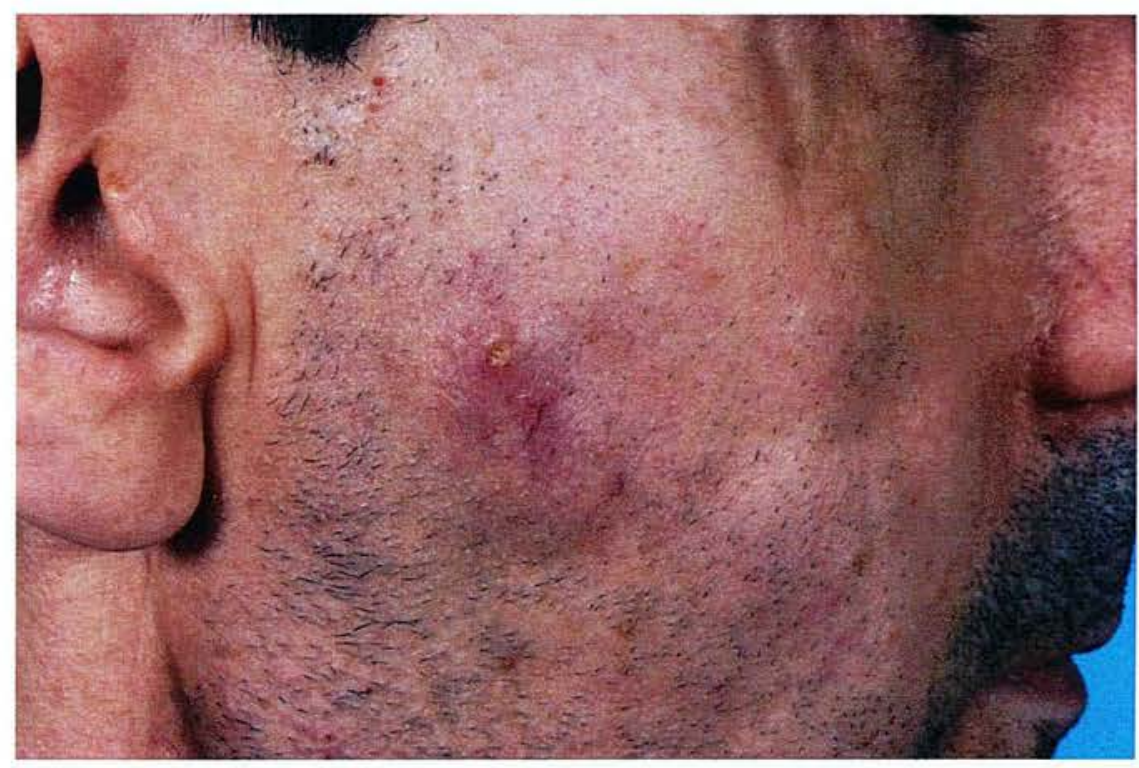

Therefore, all remaining Bio-alcamid $\AA$ was removed from the right cheek and temple via stab incisions, curettage and irrigation. The patient refused removal of the product from the left side of his face and long-term oral metronidazole therapy was continued. Four months later, the areas of inflammation remained quiescent but resulted in undesirable facial scarring (Figure 2.7). 
Figure 2.7. Post-operative scarring following removal of Bio-alcamid

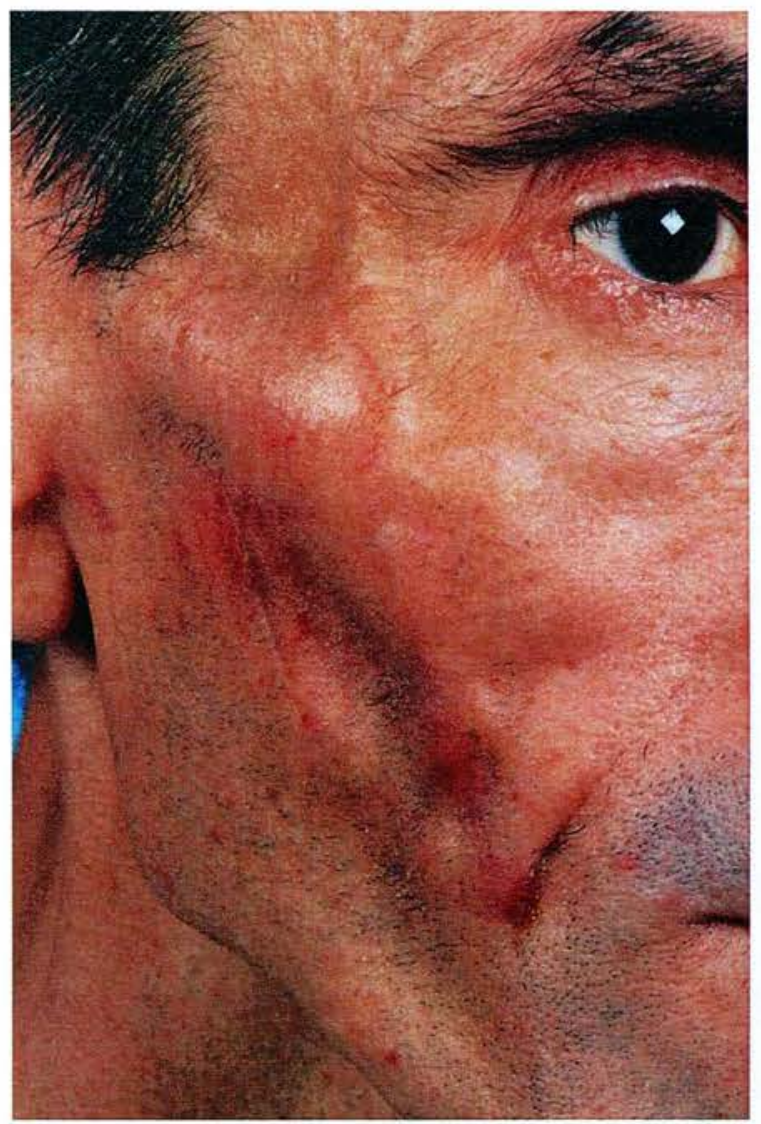

In addition, the patient also developed problematic metronidazole-induced paraesthesia.

Extrusion of Bio-alcamid to the buccal mucosa of the right cheek was seen in one patient, 6 months following 2 revision procedures to correct minor asymmetry (Figure 2.8). Manual expression of Bio-alcamid via the fistula tract combined with antibiotic prophylaxis was attempted on two occasions. However, the patient subsequently required removal of all remaining product via stab incisions and curettage. 
Figure 2.8. Extrusion of Bio-alcamid

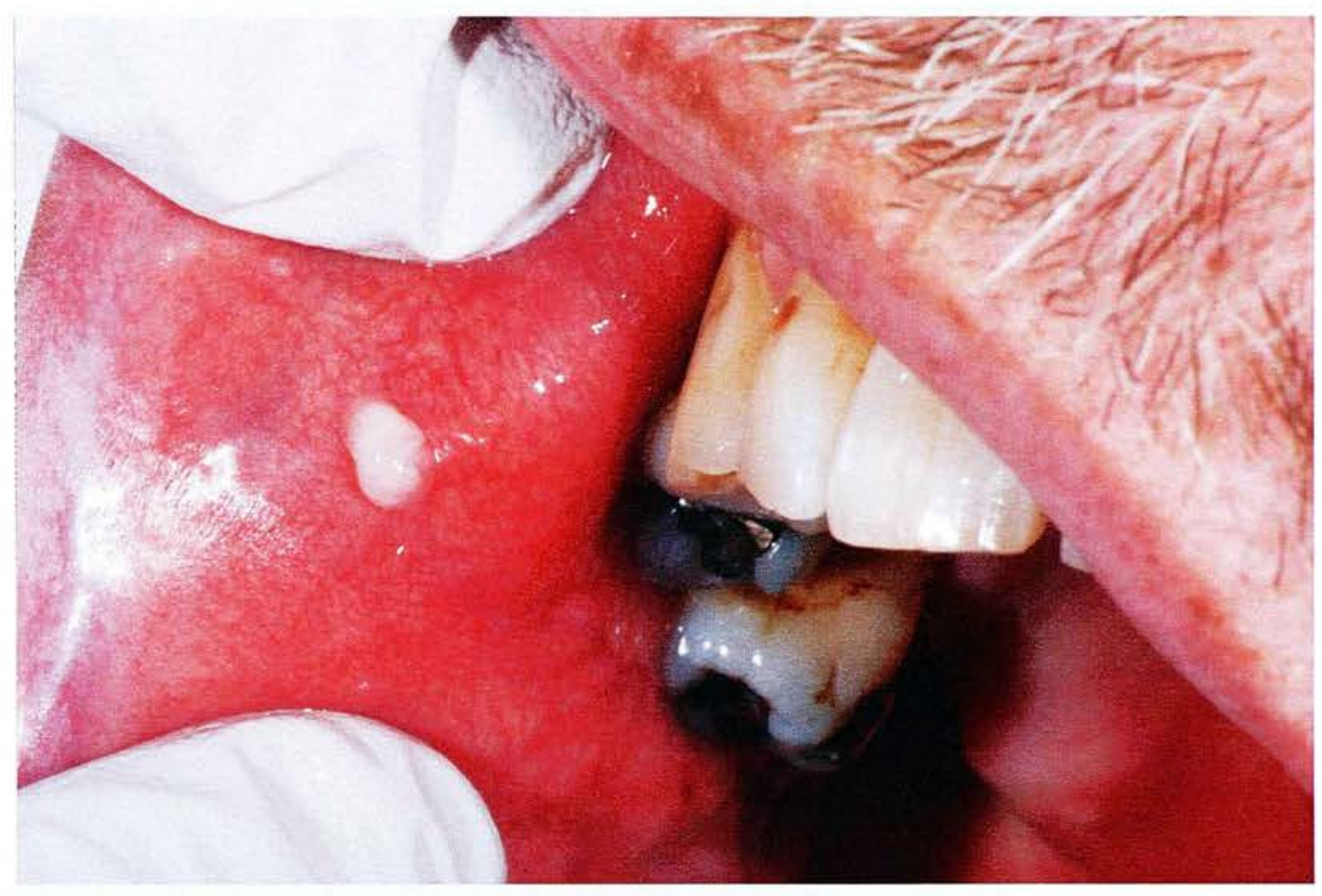

Inferior migration of the product from the malar region to the jowls occurred in 3 patients, 12 months to 3 years following treatment, and required further surgery (Figure 2.9).

Two patients developed firm, non-inflammatory swellings in treated areas which persisted despite aspiration of the product, and excess capsule formation was suspected to account for this contour abnormality. 
Figure 2.9. Migration of Bio-alcamid

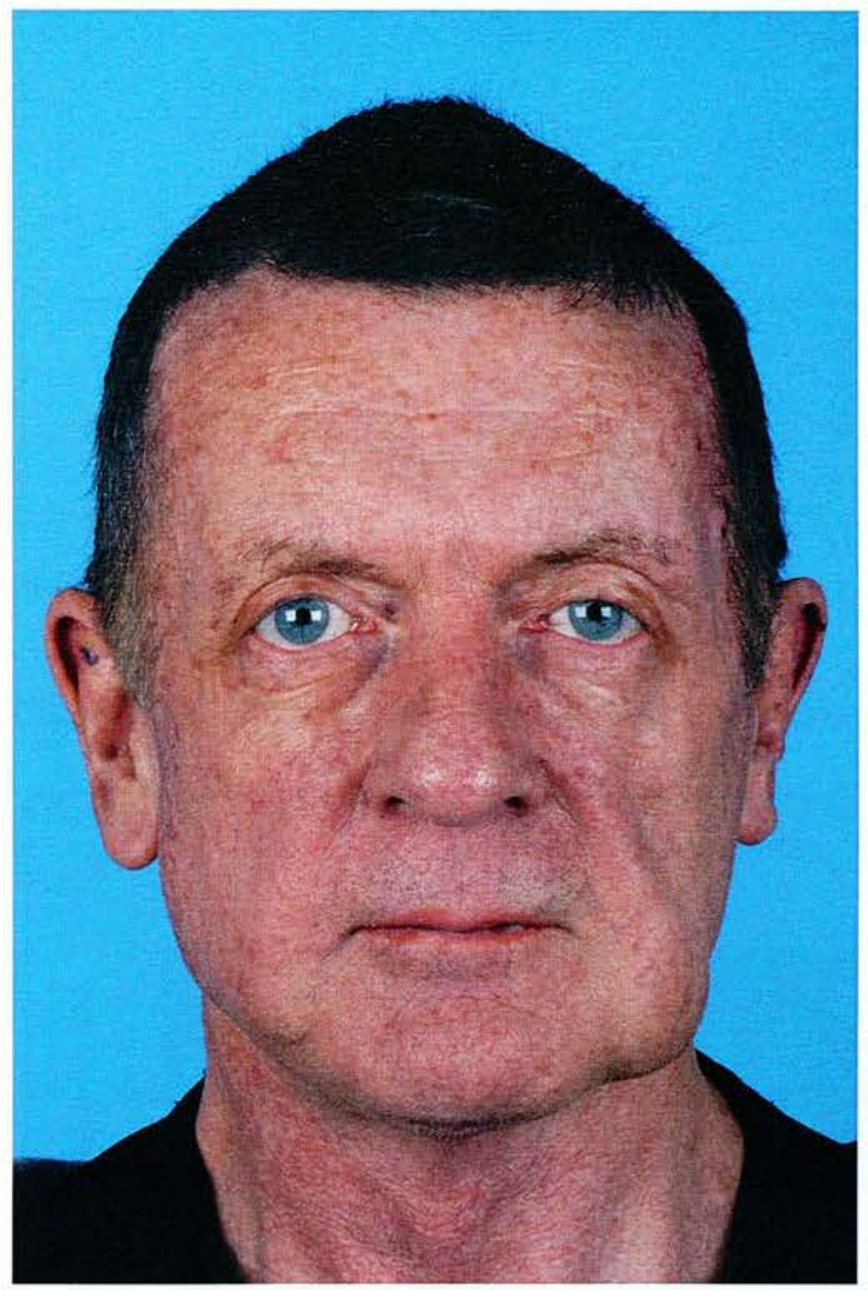

\subsubsection{Newfill}

Patients treated with Newfill experienced mild injection-related side-effects of

treatment such as swelling and or bruising which settled within one week. There were six patients who developed palpable subcutaneous nodules but these were not visible and therefore did not require treatment. Three patients complained of minor asymmetry in treated areas.

Table 3.13 and Figure 2.10 show the number of treatment sessions and top-up treatments for Newfill. 
Table 3.13. Summary of Newfill sessions

\begin{tabular}{|r|r|r|}
\hline & Newfill & Top-up \\
& session & session \\
& $\mathrm{s}$ & $\mathrm{s}$ \\
\hline Mean & 5.4 & 3 \\
(SD) & $(2.3)$ & \\
\hline
\end{tabular}

Figure 2.10. Summary of Newfill treatment sessions

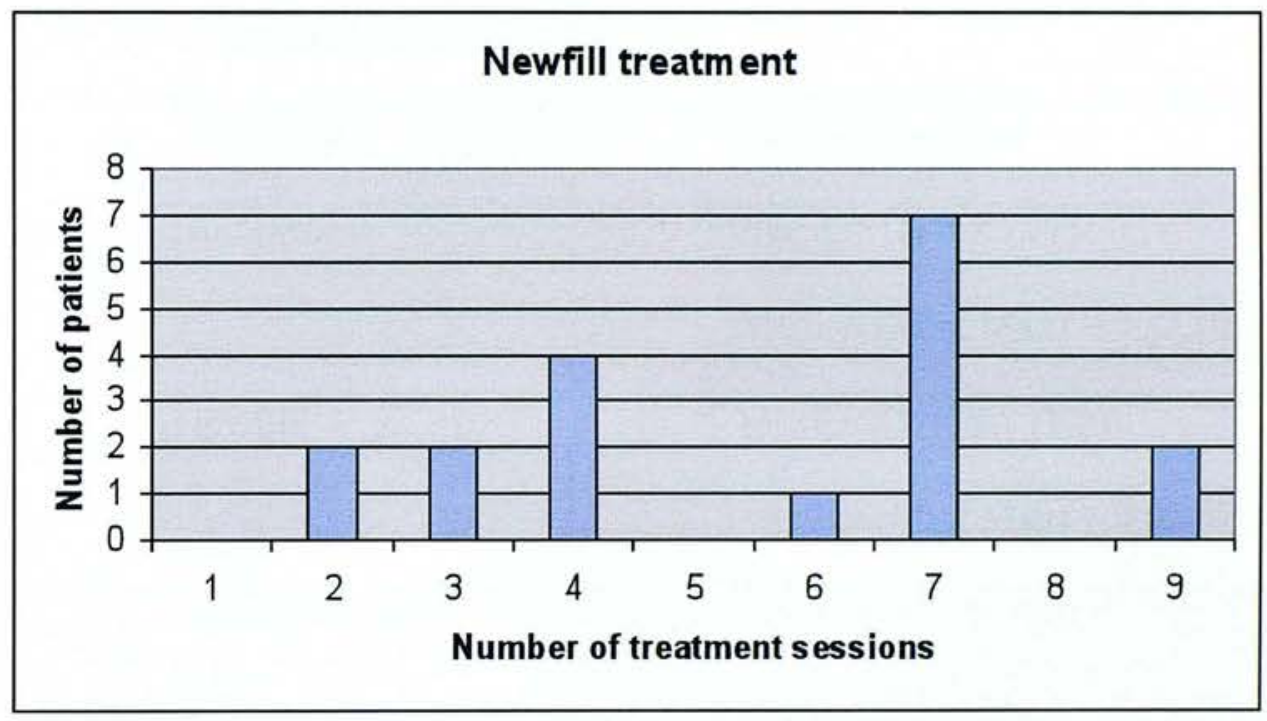

Seven treatment sessions were required for almost $50 \%$ of patients. Two patients with severe lipoatrophy required nine treatment sessions and still felt that the volume augmentation achieved was insufficient. Two patients did not complete a course of treatment due to personal or social circumstances therefore full correction of lipoatrophy was not achieved. Three patients required a top-up injection 18-24 months following completion of treatment for minor areas of subjective volume loss. 


\subsubsection{Autoiounousfát transfer}

There were few comptications associated with autologous fat transfer apart from mild sweiling and or bruising of the treated areas and donor sites whichiresolved within 22 weeks: Based on subjeetive / findings and chinical assessment, four patreats underwent: resorption of transferred fat: Two patients were subsequently treated withiNewitl and one patient opted for treatment with-Bio-alcamid:

\subsubsection{Pratient sadisfactiom with treatment}

Tabie:225summarizes theresults of patient satisfaction with treatment for each: treatment group.

Tabie:2.55. Patient satisfactionscores:

\begin{tabular}{|c|c|}
\hline Treatment: & Satisfactionscore \\
\hline Bio-edcamid & $\begin{array}{l}\text { Méan }(\mathrm{SD})=8: 7^{-}(1) \\
\text { Médian-(Min; Max) }=9(7,10)\end{array}$ \\
\hline Newfiill & $\begin{array}{l}\text { Mean }(S 4)=887^{7}(1.33) \\
\text { Median }(\text { Nin, Max })=99(6,10)\end{array}$ \\
\hline Autologous:att: & $\begin{array}{l}\text { Meam }(S \mathrm{D}))=9(0.89) \\
\text { Wediam (Nin, Wáx) }=9(8 \$ 10)\end{array}$ \\
\hline
\end{tabular}

Examples of pre-andipost teperative 32-D) clinicat images for teach treatment method are stiown in fig̣ures:22111-22133. 
Figure 2.11. Pre and 6-month post-operative result for patient treated with fat
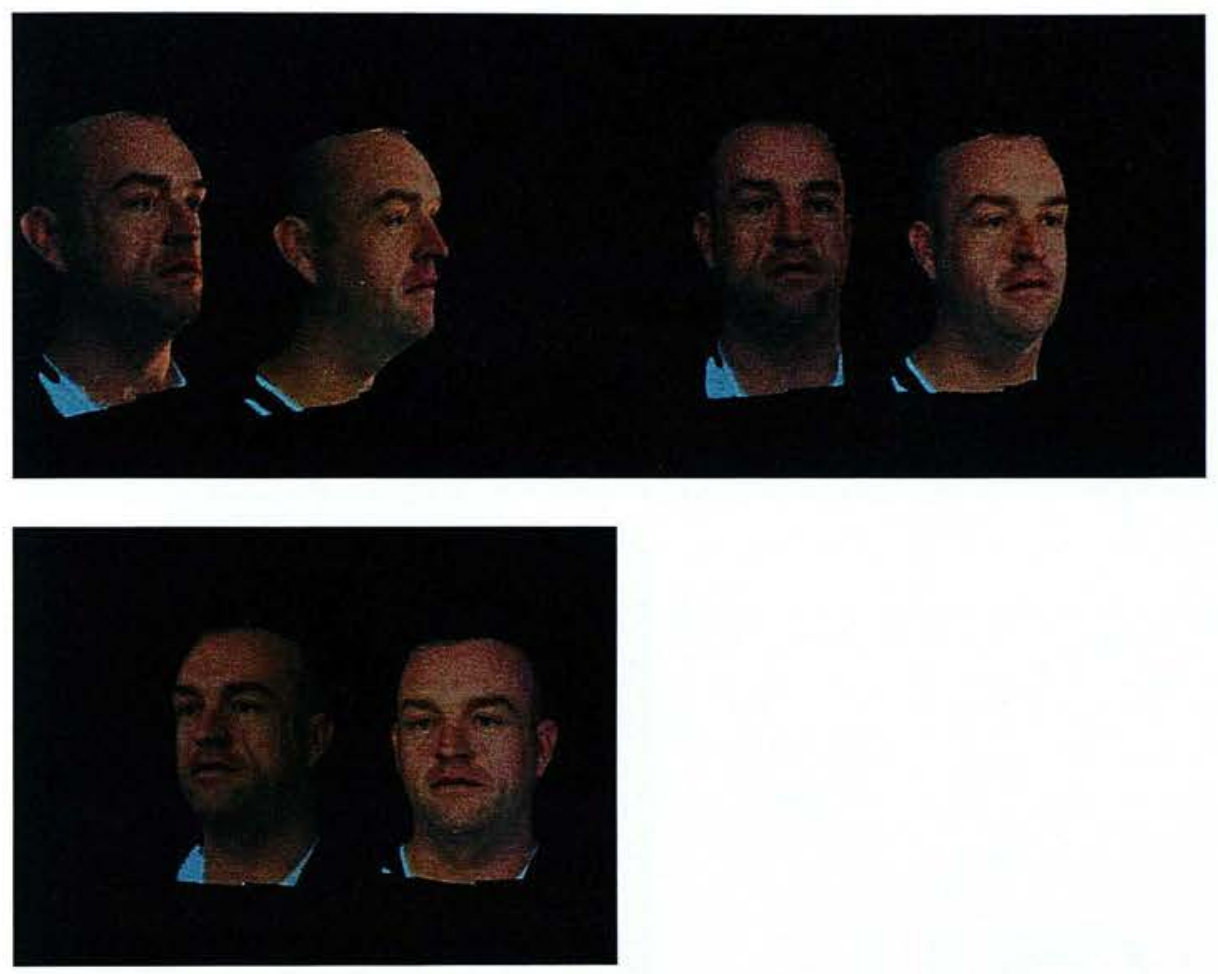

Figure 2.12.. Pre and 6-month post-operative result for patient treated with Newfill

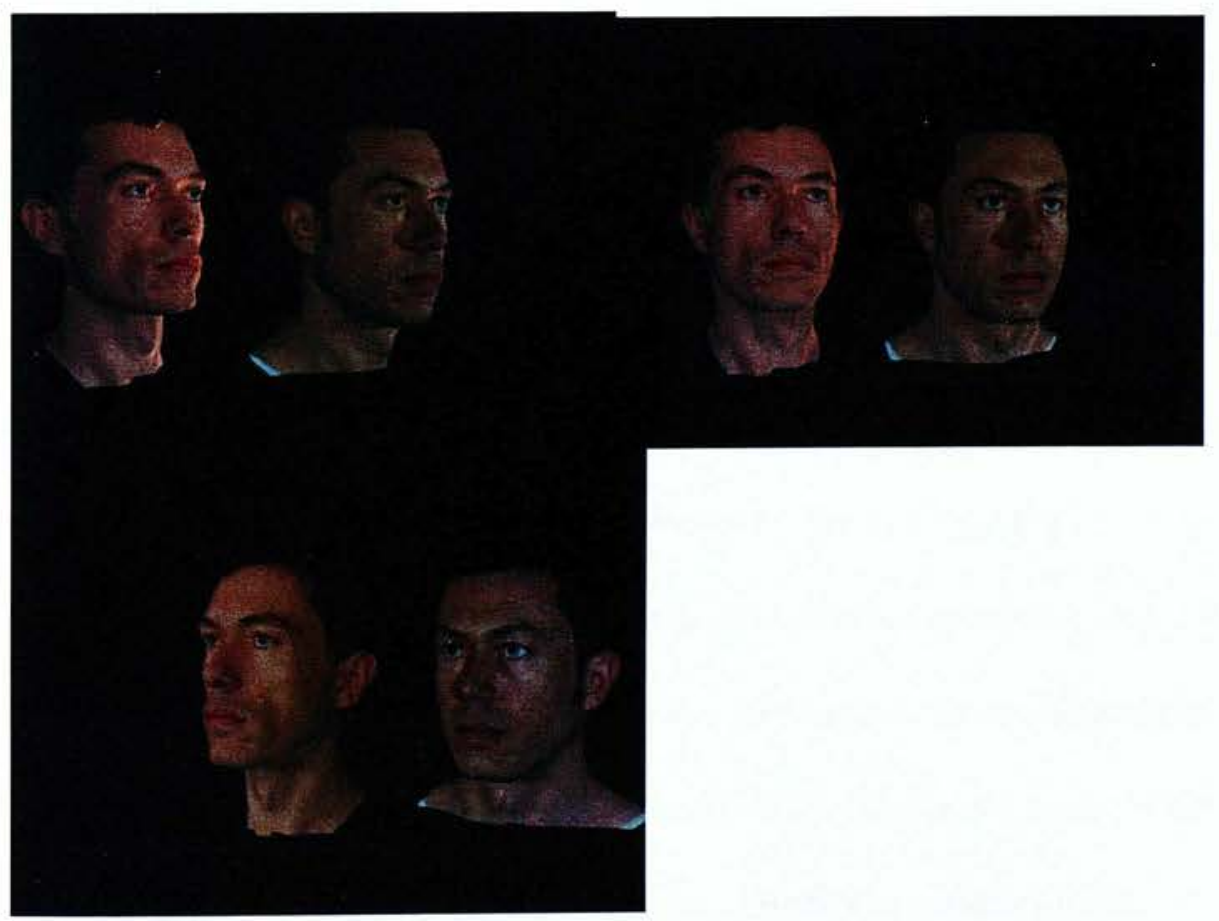


Figure 2.13.. Pre and 6-month post-operative result for patient treated with Bio-alcamid
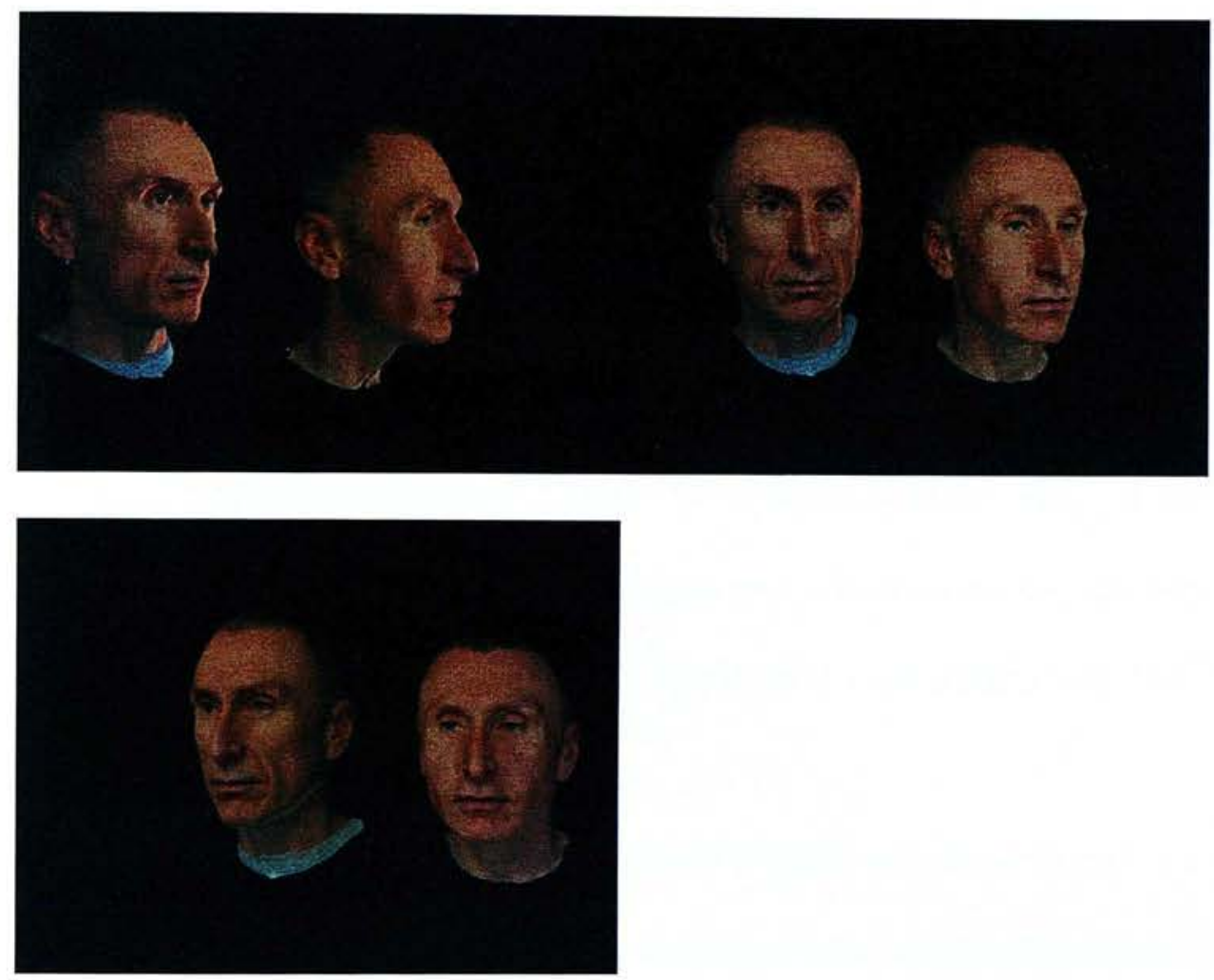

\subsection{Discussion}

\subsubsection{Baseline characteristics of treatment groups}

The 3 groups of patients undergoing treatment for facial lipoatrophy were reasonably well matched with regards to age, and haematological parameters. However, there were some important differences between the groups which shall be discussed in this section. The treatment numbers varied slightly between groups with the smallest number of patients undergoing fat transfer and the largest number receiving Newfill injections. The clinical pattern of lipodystrophy was a key factor determining choice of treatment. Only patients with sufficient autologous donor fat (including areas of lipohypertrophy) were selected to the AFT group. Thus, the majority of patients in the AFT group had a mixed morphological pattern. This was also reflected in the higher BMI and skin fold 
thickness measurements in this group. Patients with insufficient subcutaneous fat for transfer were offered treatment with a filler. Sixty-five percent of the Newfill group and $50 \%$ of the Bio-alcamid group presented with lipoatrophy alone. Although a mixed morphological pattern was seen in some patients, the fat distribution was not appropriate for fat transfer (e.g. intraperitoneal fat). In addition, 3 patients requested Bio-alcamid treatment and 2 patients requested Newfill treatment following complete resorption of previously transferred autologous fat. Thus, patient preference was also a key factor when considering treatment options. Although all 3 treatments were discussed with patients, 9 out of 11 patients recruited from the Glasgow unit requested Newfill therapy. This may have been influenced by local resources and bias from the referring units as all patients with facial lipoatrophy were treated with Newfill in Glasgow prior to the study.

Overall, a greater number of females with HIV lipodystrophy presented for treatment than predicted compared to previous national and local studies. This introduced further variability in treatment numbers as differences in clinical pattern were observed between genders. Eighty-seven percent of females presented with a mixed morphology compared to $48 \%$ of males, which explains the slightly larger proportion of females in the AFT group. This study may provide some evidence to support the hypothesis of different clinical patterns of lipodystrophy observed between genders. It has been observed that females tend to develop a mixed pattern of lipodystrophy or lipohypertrophy compared to a greater proportion of lipoatrophy in males. This may be related to hormonal influences on fat distribution between genders. However, the pathogenesis is likely to be multi-factorial and previous studies have not demonstrated a direct correlation between morphological pattern and gender.

A difference in baseline clinical grading of facial lipoatrophy was also seen, with significantly higher scores in the Bio-alcamid group compared to the AFT and Newfill 
groups. The study was designed to randomize patients who required a filler to receive treatment with either Newfill or Bio-alcamid. However, the majority of patients refused randomization and had a preference for their choice of treatment. This was largely due to the difference in properties of the two fillers. Consequently, patients with more severe facial lipoatrophy tended to favour treatment with Bio-alcamid which can be injected in large volumes to achieve a full and permanent correction.

\subsubsection{Complications of treatment}

Clinical follow-up in many patients extended beyond the 12-month trial interval. This was important to evaluate longer-term adverse effects of treatment and the complications which emerged following Bio-alcamid injections became a significant concern. Early in-vitro studies evaluating the use of Bio-alcamid emphasized the safety and biocompatibility of the product (Ramires, Miccoli et al. 2005). The largest multicentre study of 2000 otherwise healthy patients reported excellent cosmetic results with only 12 complications of $S$. aureus infection (Pacini, Ruggiero et al. 2002). This study also reported no observed cases of migration, dislocation, granulomas, allergic response or intolerance of the implant. However, the true incidence of complications relating to Bio-alcamid is likely to be underreported due to the large number of patients treated by cosmetic practitioners with insufficient follow-up. During the trial period, reports have emerged from plastic surgery units which have become referral centres for complications relating to the use of this Bio-alcamid. The greatest concern, particularly in HIV positive individuals, is the potential for infection. A number of safety studies have shown Bio-alcamid ${ }^{\circledR}$ to be non-toxic, non-allergenic, non-reactive, biocompatible and non-biodegradable (Pacini, Ruggiero et al. 2003; Formigli, Zecchi et al. 2004). However, despite evidence of its biocompatibility, Bio-alcamid® still carries the risk associated with implants to provide a favourable environment to harbour bacteria. 
Jones et al have emphasized the late appearance of abscesses months to years after injection of the material (Jones, Carruthers et al. 2007). In some cases, the onset of infection was triggered by additional revision procedures at or near the site of previous injections (Karim, Hage et al. 2006). However, complications may also arise spontaneously without any history of trauma or local infection. In most published cases, microbacterial cultures have demonstrated streptococcal or staphylococcal bacteria. Interestingly, Clostridium perfringens, an anaerobic bacteria commonly associated with food borne illness, was isolated in one of our patients. Management of infection by antibiotic therapy or attempted aspiration is often unsuccessful. Surgical incision and drainage is usually required to remove all infected material resulting in undesirable facial scarring. A technique of drainage utilising an irrigation system has been described by one institution to minimise skin incisions (Goldan, Georgiou et al. 2007). The potential for infection-related complications is a disastrous and unacceptable result for patients seeking cosmetic enhancement. Because spontaneous inflammatory reactions are possible years after injection, it has been suggested that Bio-alcamid $\circledast$ may lead to chronic interference with surrounding tissues and enhanced susceptibility to infections (Alijotas-Reig, Garcia-Gimenez et al. 2008). Thus, immunocompromised patients with HIV infection may represent an even more vulnerable patient group. Although warnings against the use of Bio-alcamid $\circledast$ for cosmetic indications have been published (Karim, Hage et al. 2006), Bio-alcamid® is still considered to have a role in the management of severe reconstructive deficits such as HIV lipodystrophy. However, the results of this trial demonstrate that the product should be used even more cautiously in this patient group.

Bio-alcamid $\circledast$ is also prone to other risks associated with prostheses such as migration and capsule contraction. Capsule formation around the implant is suggested to prevent 
migration by providing anchorage with surrounding tissues (Formigli, Zecchi et al. 2004). However, gravitational migration was evident in 3 of our patients and is perhaps associated with injection of large volumes of the material into mobile areas. It has been suggested that filler migration may occur after manipulation of the product with disruption of the surrounding capsule (Ross and Malhotra 2009). However, marked migration of Bio-alcamid $\AA$ was observed in patients who have not undergone revision procedures. Areas of excessive capsule formation can also be unpredictable and difficult to treat.

The economic burden of complications relating to Bio-alcamid® must also be considered. The requirement for in-patient admission, intravenous antibiotic therapy, surgical drainage and further corrective procedures may incur significant costs to health trusts. Although private cosmetic practitioners carry out many of these procedures, patients with complications often present via acute hospital settings seeking treatment. In addition, some cosmetic practitioners lack the facilities or skill to address complications requiring surgical removal.

Therefore, the potential for significant short and long-term complications that have emerged as a result of the trial have led to a change in management of patients with HIV lipodsytrophy in the Edinburgh unit. Bio-alcamid is no longer offered as a treatment option and alternative treatments are being evaluated to rectify some of the on-going problems.

There were no adverse events following treatment with autologous fat transfer. The selective randomisation methodology of this study was chosen in the belief that autologous fat transfer is the most physiologically compatible filler, and the results of the study support the safety of this treatment. No evidence of lipohypertrophy of transferred fat was seen in this patient group, despite harvesting fat from the DCFP in 
migration by providing anchorage with surrounding tissues (Formigli, Zecchi et al. 2004). However, gravitational migration was evident in 3 of our patients and is perhaps associated with injection of large volumes of the material into mobile areas. It has been suggested that filler migration may occur after manipulation of the product with disruption of the surrounding capsule (Ross and Malhotra 2009). However, marked migration of Bio-alcamid $\AA$ was observed in patients who have not undergone revision procedures. Areas of excessive capsule formation can also be unpredictable and difficult to treat.

The economic burden of complications relating to Bio-alcamid® must also be considered. The requirement for in-patient admission, intravenous antibiotic therapy, surgical drainage and further corrective procedures may incur significant costs to health trusts. Although private cosmetic practitioners carry out many of these procedures, patients with complications often present via acute hospital settings seeking treatment. In addition, some cosmetic practitioners lack the facilities or skill to address complications requiring surgical removal.

Therefore, the potential for significant short and long-term complications that have emerged as a result of the trial have led to a change in management of patients with HIV lipodsytrophy in the Edinburgh unit. Bio-alcamid is no longer offered as a treatment option and alternative treatments are being evaluated to rectify some of the on-going problems.

There were no adverse events following treatment with autologous fat transfer. The selective randomisation methodology of this study was chosen in the belief that autologous fat transfer is the most physiologically compatible filler, and the results of the study support the safety of this treatment. No evidence of lipohypertrophy of transferred fat was seen in this patient group, despite harvesting fat from the DCFP in 
one patient. The best choice of donor site remains a topic of debate. Some authors propose that fat harvested from areas of lipohypertrophy may have some survival advantage (Davison, Timpone et al. 2007). However, the only patient in this study who had fat harvested from the DCFP underwent complete fat resorption, which may relate to the poor quality of fat deposited in these areas. New theories regarding protein receptor status of adipocytes within regions of lipohypertrophy have been proposed. Guallar et al have demonstrated a high expression of proliferating cell nuclear antigen (PCNA), a marker gene of cell proliferation, in adipocytes harvested from the buffalo hump region (Guallar, Gallego-Escuredo et al. 2008). The authors suggest that a transformed phenotype in cells from these sites develop an intrinsic enhancement in cell proliferation, which remains even when transferred to lipoatrophic sites. The study also demonstrated variability in PCNA expression, and therefore proliferative capacity, in different buffalo hump samples. Although further investigation in this field is required, it may explain why buffalo hump deformity may recur following treatment with liposuction. It may also provide an explanation for the well-documented 'hamster cheek' syndrome which may develop following transfer of buffalo hump fat adipocytes to treat areas of facial lipoatrophy (Guaraldi, De Fazio et al. 2005).

Variable degrees of fat resorption were demonstrated post-operatively, but whether this is considered a complication or rather an anticipated side-effect of treatment is questionable. The degree of resorption of autologous fat grafts also remains a topic of debate, and is mainly based on subjective observation of surgically treated areas over time. Indeed, studies are difficult to compare due to the wide variability in technique, experience of the surgeon and means of evaluating outcome. Compared to other nonpermanent fillers, the longevity of autologous fat is not as easily predictable. For patients with HIV lipodsytrophy, the unpredictable nature of the technique is even more apparent due to the underlying systemic lipodystrophic process. 
The perceived success of autologous fat transfer depends on whether one considers long-term resorption disappointing or potentially superior to the anticipated longevity of other non-permanent fillers. Thus, the less predicable long-term volume augmentation achievable with autologous fat must be balanced against the risks of allergic responses and other complications relating to synthetic injectable fillers. The simplicity and safety of autologous fat transfer also makes it an attractive option compared to other surgical options for HIV facial lipoatrophy including the use of dermis-fat grafts, flaps and implants.

Newfill therapy was also found to be a safe treatment in this study. Six patients developed palpable subcutaneous nodules, although these were not visible and did not require treatment. Subcutaneous nodules are the most commonly reported side-effect of Newfill treatment (occurring in $52 \%$ of patients in the VEGA study.) Nodules can be visible or nonvisible (ie only palpable) and can be temporary or permanent. It has been suggested they can arise as a result of technical error, uneven distribution in the suspension, or uneven dispersal in the injected areas. Histologically, the nodule consists of fluid droplets surrounded by a normal foreign body reaction. Nodules can be further subclassified into early and late nodules (Lam, Azizzadeh et al. 2006). All of the patients in this study developed early nodules which typically appear within several weeks of treatment. They can be disrupted by needle fragmentation of the nodule and injection with saline to hydrate and redistribute the particles followed by aggressive massage (Lam, Azizzadeh et al. 2006). If the nodules do not resolve over time, they can be locally excised. Late nodules that appear several months after injection may be treated with an intralesional steroid (triamcinolone, $10 \mathrm{mg} / \mathrm{cc}$ ) and massage (Vleggaar 2006). Again, failure to respond to treatment may require local excision of the lesion. 
The guidelines for reconstitution of Newfill have recently been modified by the manufacturers to minimise the risk of nodule formation. These include increasing the volume of saline when reconstituting the product from 5 to $10 \mathrm{cc}$. It is also suggested that the sterile water should be added $24-48$ hours before injection to allow sufficient time for the product to be entirely reconstituted (Vleggaar and Bauer 2004).

\subsubsection{Patient satisfaction}

Mean patient satisfaction was similarly high for all 3 treatments. However, there are various weaknesses of the method that should be highlighted. The inclusion of numbers as well as the word descriptors on the VAS led to patients indicating their response with a number on the horizontal line. Although a numerical value was useful for collating scores and calculation of mean values, the scale was not a true VAS as it did not capture patient perspective along a continuum. The inclusion of the numbers implied specific metric relations and therefore only pre-specified levels could be given by the respondent. This was a flaw in the design of the VAS and the numbers should have been omitted. One of the limitations of the scale included the 'ceiling effect' with many patients scoring their satisfaction at the top end of the scale. Patient satisfaction scales designed in this way are difficult to interpret because responses are a complex sum of expectations that may vary among patients with comparable care. Many factors unrelated to the surgery may cloud the perception of patient satisfaction, for example unfavourable interpersonal relationships during the treatment period (patient-physician or nursing staff). Although an attempt to measure some different elements of patient satisfaction was undertaken in this study, these factors were not comprehensive and the scales were non-validated. Although some validated and reliable satisfaction instruments shave been developed in other fields such as primary care and palliative care, these have limited application to aesthetic surgery. Following review of the 
literature (Ching, Thoma et al. 2003), no appropriate tools to directly evaluate patient satisfaction in aesthetic surgery were found. Most studies rely on scales developed by the researchers themselves. Therefore, there is a need for development of an instrument to measure satisfaction with facial appearance and quality of life following aesthetic appearance. This is particularly important given that patient satisfaction is the main factor in aesthetic surgery that dictates success of the procedure. Technically perfect results may be considered a failure if the patient is not satisfied.

When interpreting the results in this study, there are further elements of bias to consider. Firstly, there is a high likelihood of bias from patients reporting satisfaction to their surgeons. Indeed, some patients scored satisfaction highly despite development of complications or an imperfect aesthetic outcome. This may imply that they found other elements of the treatment process satisfactory or that they were motivated by potential secondary gain to receive further treatments. An additional factor to consider is that patient satisfaction scores were collected 2 to 6 months following treatment so may not represent the opinions of the patients who subsequently developed later problems with Bio-alcamid. The inclusion of a further patient satisfaction score at the end of the trial follow-up or after completion of re-treatments would have been beneficial.

\subsection{Conclusions}

This study demonstrates baseline demographic and clinical data in patients undergoing treatment for HIV facial lipoatrophy. High patient satisfaction was seen following treatment in each group although scores do not reflect long-term satisfaction. The study highlights the complications associated with the chosen treatments, particularly in the Bio-alcamid group, and this has led to withdrawal Bio-alcamid as a treatment option in the Edinburgh Unit. Further comparison of treatment groups with the addition of 
measured 3-D and psychological outcome data are provided in the overall discussion and conclusions. 


\section{Chapter 3. Three-dimensional facial volume analysis in HIV lipodystrophy}

\subsection{Aims}

1. To evaluate facial volume change in patients undergoing treatment for HIV associated facial lipoatrophy.

2. To validate the use of the DI3D system as a tool for measuring volumes of anatomical areas affected in patients with HIV lipodystrophy.

\subsection{Method}

\subsubsection{Design}

A prospective, follow-up study was designed to assess volume change in surgically treated areas following 3 different interventions: autologous fat transfer, Newfill and Bio-alcamid.

\subsubsection{Sample}

A cohort of 48 patients with HIV facial lipoatrophy were recruited from Plastic Surgery clinics in Edinburgh and Glasgow. All patients underwent clinical assessment by 2 doctors (a Consultant plastic surgeon and the lead researcher) to identify features of lipodystrophy and select choice of treatment. Patients with sufficient subcutaneous fat were offered treatment with autologous fat transfer. Those patients with insufficient fat due to generalized lipoatrophy were offered treatment with either Newfill or Bioalcamid.

A complete description of the recruitment process and details of surgical technique for each treatment is provided in Chapter 2.

\subsubsection{Outcome measures}


Measurement of facial volume was carried out using the Di3D system and software. Images were captured on all patients pre-operatively, within a 2-week interval prior treatment. Repeat images were performed at 2,6 and 12 months following completion of treatment.

In addition, patients underwent clinical follow-up and assessment of psychological outcomes at similar time intervals. These outcomes are presented in the relevant chapters.

\subsection{DI3D Facial System}

\subsubsection{Data Capture}

\subsubsection{Calibration}

Calibration was carried out prior to each imaging session within an hour of the subject's appointment. This calibration was based on photogrammetric techniques and allowed the detailed geometric configuration of all the cameras to be determined. A calibration target was positioned $91 \mathrm{~cm}$ from the DI3D system and comprised a grid of accurately known dimensions containing 10 x 10 black discs on a white background. The calibration target was captured by the cameras in three different positions and at two different angles. Images were captured with the calibration target in the Frankfurt plane then at an angle of +30 degrees to the Frankfurt plane and the calibration target positioned directly facing the base of the unit then repositioned in the horizontal plane to face the right and left pods respectively. DI3D then calibrated the system and reported a calibration error which was always below $0.4 \mathrm{~mm}$.

During the calibration process, which took around 5 minutes, images of the target from all the cameras were processed to find the central location of the discs and these coordinates were used to fit an approximate geometric model of each camera and its respective relative orientation to the target. 


\subsubsection{Subject capture}

Prior to imaging, subjects completed a medical illustration consent form.

Subjects were asked to remove spectacles and jewellery and to change into a blue theatre gown. Subjects with long hair were given a hair band so that all of the face to the hairline was visible. Once seated in a wheeled chair in front of a blue background, subjects were then positioned $91 \mathrm{~cm}$ from the DI3D system using a tape measure. The measurement was taken from the lateral aspect of the right eye to the base of the system. Subjects were asked to look directly at a cross marked on the midpoint of the base and levers were used to move the base relative to the tripod stand such that crosses viewed through the camera lens were aligned with the subjects' pupil. Subjects were asked to adopt a neutral facial expression with lips placed together.

Using the DI3D Capture application on the laptop computer, the four cameras capture the image simultaneously within seconds. An additional flash positioned behind the DI3D system and directed at the subject was used to illuminate the scene.

In most instances, a minimum of two images were captured for each subject.

\subsubsection{Data processing}

Following each capture session the calibration file was attached and the photographs were processed to create the 3-D image via the stages described in the above section using the DI3D Capture application. The 3-D image was viewed using the DI3D View application which enabled the model to be rotated and magnified. Following selection, the data was exported to an $\mathrm{OBJ}$ file for analysis using the measurement software.

\subsubsection{DI3D View software}

The DI3D View software can be used to measure linear distances, angles, surface area and volume. The software tool for volume analysis was developed by engineers from Dimensional Imaging during the course of the study after collaboration with the lead 
researcher. A differential volume tool was developed to measure volume changes between pre-operative and post-operative images.

\subsubsection{Alignment}

The alignment of two or more 3-D images requires selection of anatomical landmarks. A landmark is a point of correspondence on each object that matches within populations (Dryden and Mardia 1998). Many soft tissue anatomic landmarks have been defined on the face. The most frequently used definitions are those by Farkas (Farkas 1994). Twelve soft tissue landmarks were selected for this study from those landmarks considered to be the most reliable in previous studies (Aung, Ngim et al. 1995; Hajeer MY 2002). Four midline landmarks and 8 bilateral landmarks were utilised. In addition, and distinguishing facial markings (such as scars, skin lesions etc) can be used as a landmark (Figure 3.1).

Anatomic landmarks were located and marked on each image by the operator and the coordinates were stored. The pre-operative and post-operative images of each patient were aligned using a technique known as Procrustes analysis. Generalised Procrustes analysis is a mathematical method of manipulating configurations of landmarks so that they can be compared independently of size and position. The configurations are first scaled to a common size and then rotated and translated to achieve a best fit (Dryden and Mardia 1998).

Following the alignment of images based on landmark localisation, the DI3D software permits the operator to further manipulate the images using translation and rotation in the $\mathrm{x}, \mathrm{y}$ and $\mathrm{z}$ axes to achieve optimum alignment of the model. 
Figure 3.1. Landmarks for image alignment

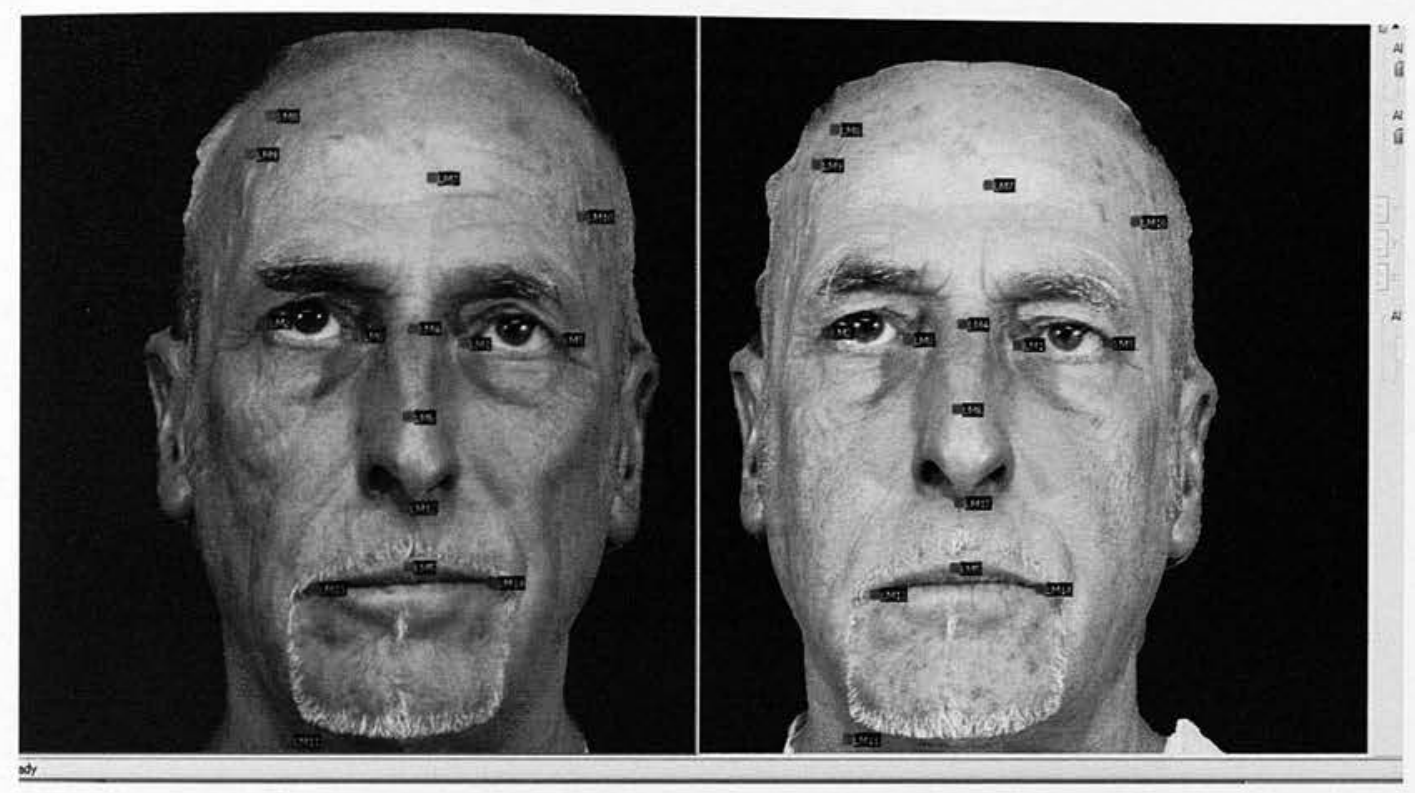

\subsubsection{Volume measurements}

Volumetric analysis of pre-operative and post-operative images were performed on the aligned model using a three-dimensional back plane positioned behind the area of analysis (Figure 3.2) The size and orientation of the plane can be manipulated in the $\mathrm{x}$, $\mathrm{y}$ and $\mathrm{z}$ axis around the defined facial area. The cheeks and temples of surgically treated patients were selected for analysis. The volume tool then calculates the volume enclosed from the back plane to the facial surface. The volume can be calculated independently for the pre-operative image and the post-operative image using the same plane. The differential volume can also be calculated automatically using a separate tool. Thus for each set of pre-operative and post-operative images, 3 volume measurements were recorded for each facial area defined by the back plane: the preoperative volume, the post-operative volume and the differential volume. 
Figure 3.2. 3-D backplane

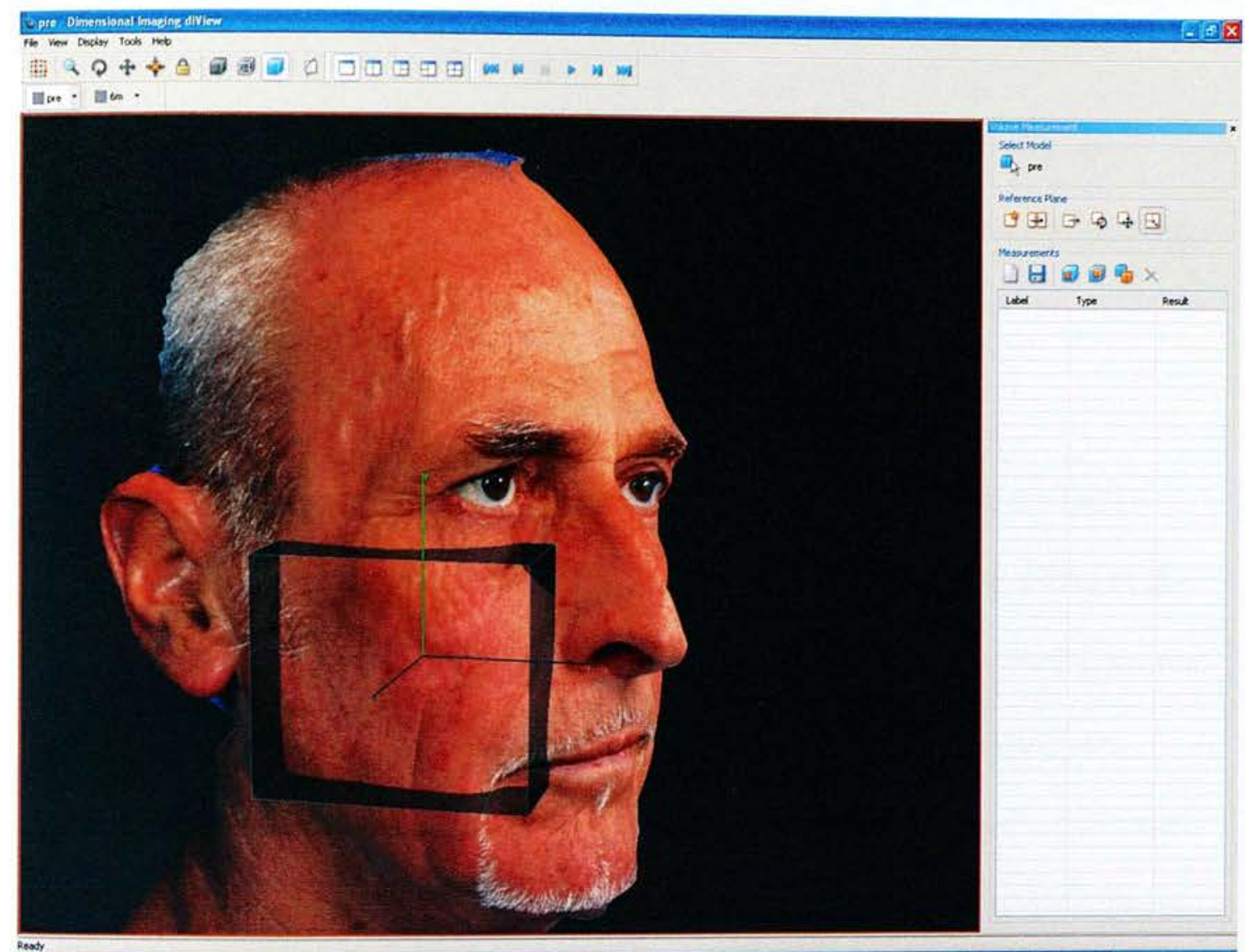

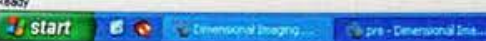

\subsection{Statistical Analysis}

Facial volumes (as differences, compared to the pre-treatment baseline 3D volume measurement), and volumes injected were summarised by treatment at baseline, and at 2, 6 and 12 months post-treatment. No baseline measurement of volume injected was available for the NewFill group. Differences between volume measurements at each follow-up visit were summarised by treatment, and within-treatment changes were tested using one-sample t-tests. Differences between groups were analysed using ANOVA. 
Pearson correlation coefficients were used to test for an association between the number of treatments received and facial volumes (compared to baseline) at 2, 6 and 12 months for patients treated with NewFill.

In addition, all data was analyzed using equivalent non-parametric tests but no differences in results were demonstrated. Thus, data is presented utilizing parametric testing as described.

\subsection{Validation of the DI3D system}

The aim of validation was to assess the accuracy and reproducibility of the DI3D system to evaluate facial volume changes. Accuracy was defined as the ability of the system to measure a given volume as close to the actual volume. Reproducibility was defined as the measure of variability between repeated trials of the imaging and measuring procedure under the same conditions. Three types of error were evaluated during validation of the DI3D system and software: operator error, reproducibility error and the DI3D system error. Three experiments were conducted.

\subsubsection{Experiment 1: Measurement Bias}

\subsubsection{Aim}

The aim of this experiment was to assess the accuracy of measurements of changes in facial volume in different regions of the face against known changes in volume.

\subsubsection{Method}

The accuracy of the DI3D system to measure facial volume changes was assessed using facial casts to eliminate sources of error from facial expression and then on human subjects. Each measurement was taken twice, to assess repeatability, and four faces were involved to assess variability between faces. 


\section{Process for facial cast and mould preparation}

Facial casts of one male and one female subject were created by a prosthetist using plaster. A plaster base was attached to the facial cast to allow the cast to sit upright on a solid surface. Twelve facial landmarks were placed on each mould using a black biro pen.

Ten flexible silicone moulds of different areas of the face were prepared from the female facial cast. Different sizes and shapes were created to represent the surgically treated areas in patients with HIV-associated lipoatrophy. Additionally, moulds of the forehead, nose and chin were produced to assess other areas of the face.

The moulds were created for the following regions of each face:

1. nose;

2. nasolabial region

3. cheek;

4. temple;

5. cheek \& temple;

6. forehead (small mould);

7. forehead (large mould);

8. malar region;

9. zygoma.

10. chin

The volume of each mould was measured by water displacement based on Archimedes' principle. Each mould was immersed in a water container at room temperature placed on a digital four-figure balance (model) then the weight of displaced water was recorded. Each mould was measured twice and the average weight was calculated. The 
volume was then calculated using the formula, volume $\left(\mathrm{m}^{3}\right)=$ mass $(\mathrm{kg}) /$ density, assuming a water density of $998.2 \mathrm{~kg} / \mathrm{m}^{3}$ at room temperature of 20 degrees celsius.

\section{Image capture}

Each facial cast was positioned on a table and captured with the DI3D system using the method described. An additional image was captured after the placement of each mould on the cast using adhesive glue. (Figure 3.3)

The process was repeated using the same male and female subjects from whom the facial casts were modelled. Twelve facial landmarks were placed on each subject using a sharp black eyeliner pencil. The face of each subject was then captured with the DI3D system using the method described. A further image was captured after the placement of each silicone mould and a 3D model was created.

The following procedure was used to calculate the volume of each mould from the 3-D images of both facial casts and human subjects using the DI3D volume software.

Figure 3.3. Facial cast with and without placement of malar silicone mould

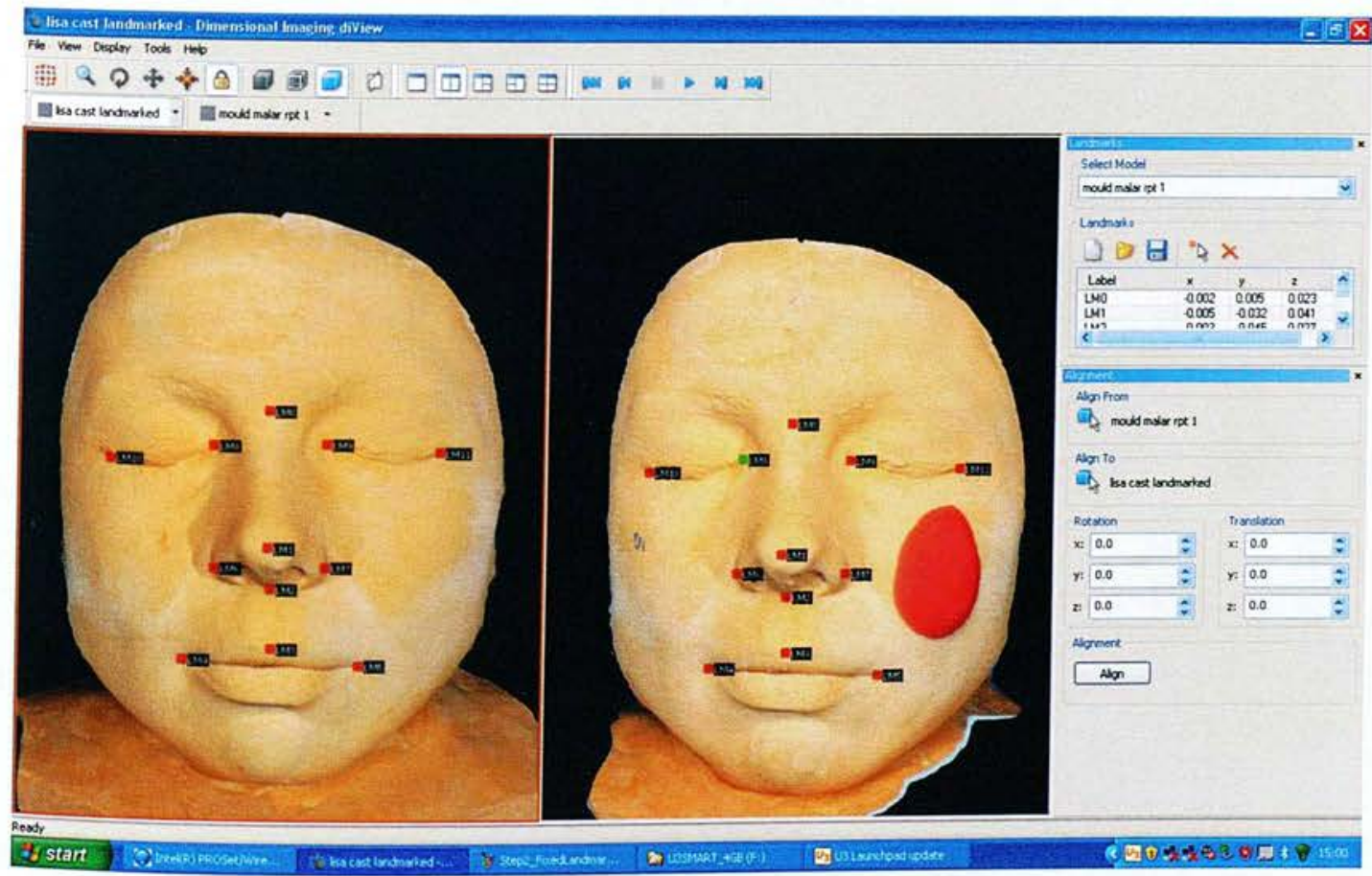




\section{Image alignment}

The 3-D models without silicone moulds were aligned to each of the 3-D models with silicone moulds using landmarked-based registration (also demonstrated in Figure 3.3). The accuracy and reproducibility of landmark localization using the DI3D system was previously assessed by comparing measurements on a plaster cast taken using a coordinate measuring machine (gold standard). (Khambay, Nairn et al. 2008) The DI3D system error was found to be within $0.2 \mathrm{~mm}$, which the authors concluded to be clinically acceptable. Magnification and rotation of images was performed by the operator to achieve accurate placement of 3-D landmarks corresponding to the 12 landmarks marked on each cast and human subject prior to image capture. The $\mathrm{x}, \mathrm{y}$ and z co-ordinates of each 3-D landmark were stored and the software then translated and rotated the two models over each other to achieve the best fit (Figure 3.4). Further manipulation of images was then performed to achieve optimum image alignment.

Figure 3.4. Image Alignment

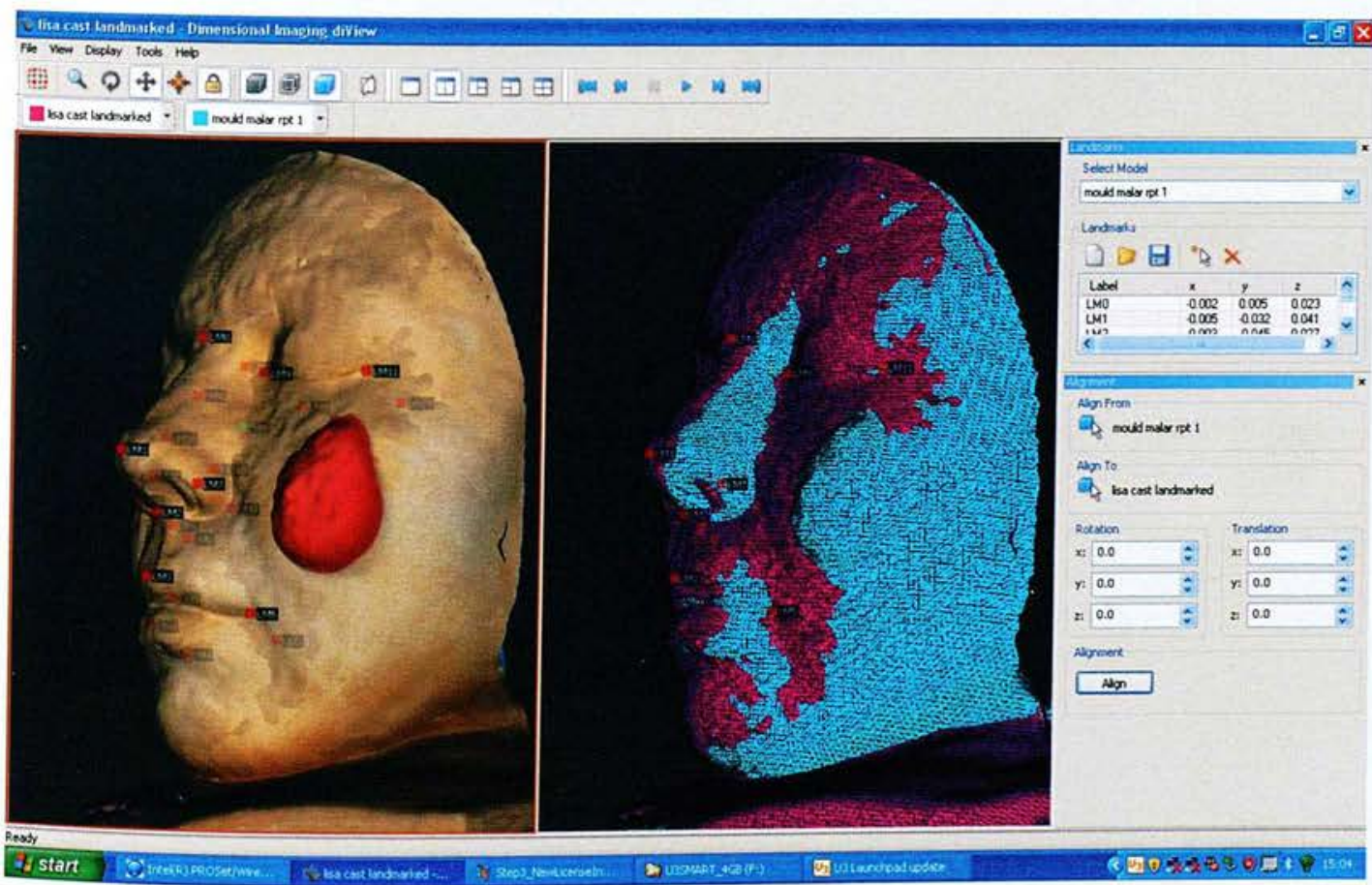




\section{3-D Volume Measurement}

The volume of each mould on the aligned image was measured using the back-plane technique previously described. The software utilizes a 3-D box as the plane which was adjusted by the operator in the $\mathrm{x}, \mathrm{y}$ and $\mathrm{z}$ dimensions to fit closely around the mould and minimize other areas of the face to be included in the volume calculation. Using the volume tool, the differential volume between the model without any moulds and each of the 10 models with a mould was measured. Volume measurements for each mould were carried out on the male and female cast and the male and female human subjects. Each volume calculation was repeated twice and the mean differential volume was recorded.

The accuracy of the system was determined by calculating the difference between volumes measured by DI3D and the actual volume of each mould measured by water displacement.

The DI3D system reproducibility and operator error were determined by measuring the variability between repeated trials of image acquisition, image alignment and volume calculation.

\subsubsection{Experiment 2: Re-Alignment and Re-Measurement}

\subsubsection{Aims}

To assess the reproducibility of image acquisition and alignment and to assess the variability of volume measurements between and within alignments.

\subsubsection{Method}

Image acquisition

To assess the reproducibility of image acquisition with the DI3D system, a mould of the malar region was placed on the female cast and was imaged 10 times within the 
same capture session. Each image was aligned to the model of the female cast without moulds and the volume of the malar mould was calculated.

\section{Image alignment}

To assess the reproducibility of image alignment, the same image of the female cast with the malar mould was realigned 10 times to the image of the female cast without moulds and the volume of mould was measured.

\section{Volume measurement}

To assess the reproducibility of the volume measurement tool, the model of the female cast with the malar mould was aligned to the female cast without moulds and a series of ten volume measurements was made using the differential volume tool, but without realignment of the image.

\subsubsection{Experiment 3: Test-Retest Reliability}

\subsubsection{Aim}

The aim was to assess the repeatability of the measurement process over a short time period.

\subsubsection{Method}

To assess variation in facial volume in the same human subject between different capture sessions, two 3-D images of 10 human subjects ( 5 male and 5 female) were captured one week apart using the same method. Twelve landmarks were placed on the face using a sharp black eyeliner pencil prior to each imaging session, and the landmarks were used to align the week one and week two images of each subject. The differential volume of three areas of the face between week 1 and week 2 was then measured. The areas measured were the forehead, right cheek and nose and the 3-D box back-plane was manipulated to enclose these areas as accurately as possible. 


\subsubsection{Statistical Analysis}

\subsubsection{Experiment 1}

For each measurement, the bias was defined as the difference between the measured volume change after adding the mould, and the known volume of the mould. The two measurements on each face made up a set of eight measurements of the volume of each of the nine moulds (results from only nine of the original 10 moulds were included). A maximum likelihood variance components model was used to estimate the components of variance due to differences between faces, moulds, time, the second-order interaction terms and residual variation. Note that variation due to moulds represents true variation; all other sources of variation are due to measurement error. The ratio of the variation due to moulds to the sum of all sources of variation therefore represents the reliability of the different measurements of the volume of each mould. The same method was applied to the eight measurement biases for the nine moulds. Figures were produced to investigate factors possibly associated with both the magnitude and variability of measurement bias, namely the true volume of the mould and the face used. These possible associations were then tested using linear regression and ANOVA methods.

\subsubsection{Experiment 2}

The two sets of measurements were standardised by subtracting the mean, and displayed using a dot plot. The variances of the two set of measurements were compared using an $\mathrm{F}$ test for equality of variance.

\subsubsection{Experiment 3}

Maximum likelihood variance components models were used to estimate the components of variance between and within subjects, separately for measurements of the forehead, cheek and nose, from which the test-retest reliabilities were estimated as 
the ratio of the between-subject variance to the total variance. A Bland-Altman graph was produced showing the difference between measurements plotted against the average of the two measurements, to assess whether the between measurement variation was associated with region or volume. Analysis of variance was used to assess whether there were any systematic difference between regions in the average differences between measurements.

\subsubsection{Results}

\subsubsection{Experiment 1}

Figure 3.5 shows the bias for all measurements, first against the true mould volume and secondly split by the four faces.

Variance components models applied to the eight measurements of volume for each mould gave an estimated reliability of $97.4 \%$, whereas the biases gave a reliability estimate of $0.0 \%$. This indicates that the eight different measurements of mould volumes were consistent, whereas the measurement biases were essentially random. Regression analysis showed the average bias to be $-0.30 \mathrm{cc}(\mathrm{p}=0.0016)$, but with no evidence of an association with true volume $(\mathrm{p}=0.12)$. There were significant differences between faces in mean measurement bias $(\mathrm{p}<0.0001)$, due to an average underestimation of the true volume for the male face $(\mathrm{p}<0.0001)$. 
Figure 3.5. Measurement bias by true volume (top) and separately for each face (bottom).
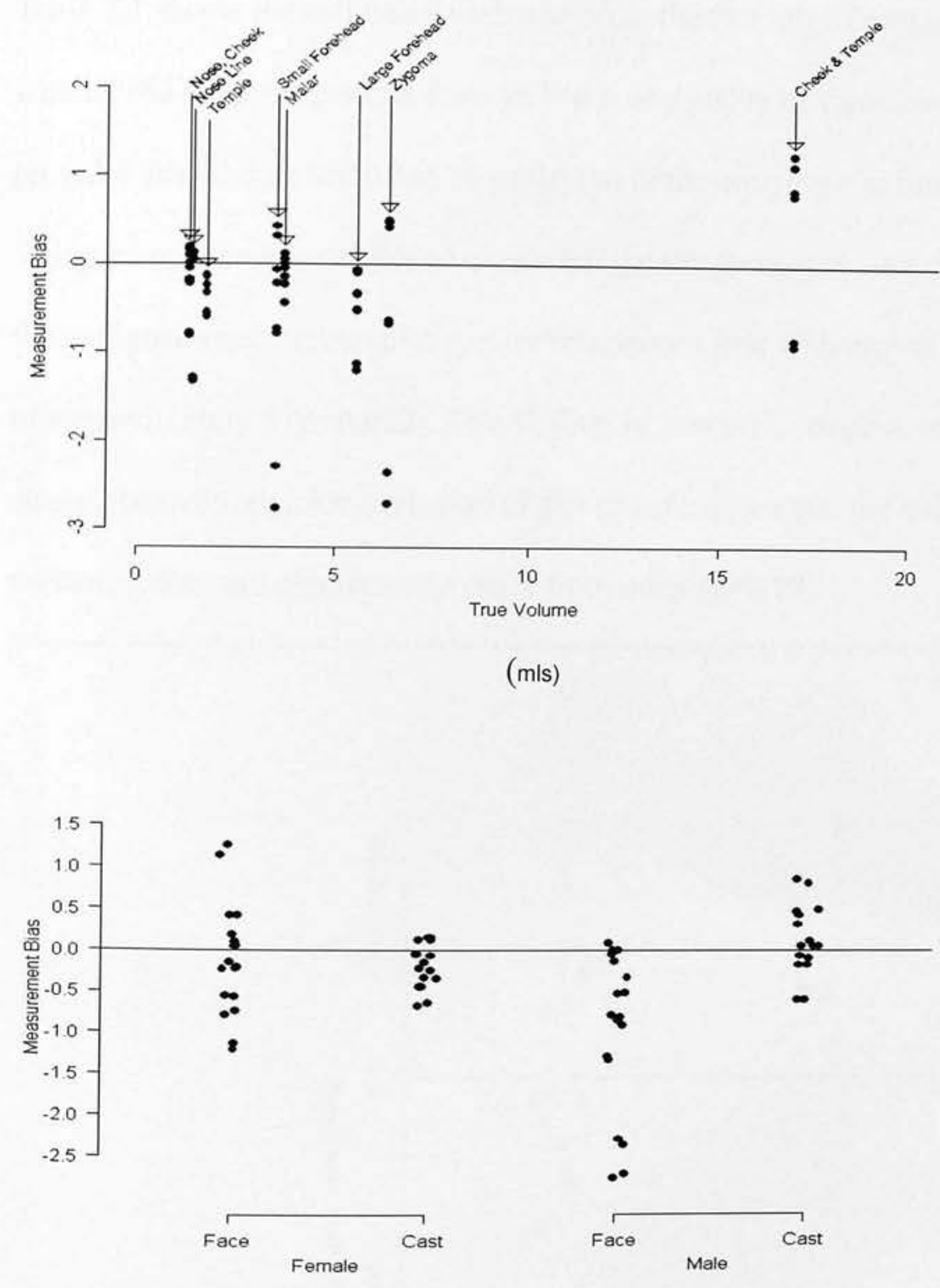

\subsubsection{Experiment 2}

Figure 3.6 shows the variation in measurements about the mean measurement for each individual, re-measured either with or without realignment of the image before each measurement. Assuming that measurements made with realignment contain error due to the realignment as well as due to re-measurement, we would expect the variation with realignment to be greater than the variation without realignment. This appears to be the 
case, though a single measurement made with realignment appears to have much greater error than all other measurements in this experiment.

Table 3.1 shows the estimated variances from the two sets of measurements, their ratio with a $95 \% \mathrm{CI}$ and a p-value from an F-test of equality of variances. This information is provided for all data and after the exclusion of the outlying measurement amongst the realigned measurements. Based on the full dataset there is evidence that the variance of the realigned measurements is greater than those made without realignment by a factor of approximately $5(\mathrm{p}=0.028)$. This finding is, however, sensitive to the inclusion of a single observation; after exclusion of this one measurement, the ratio of the two variances does not significantly differ from unity $(p=0.79)$.

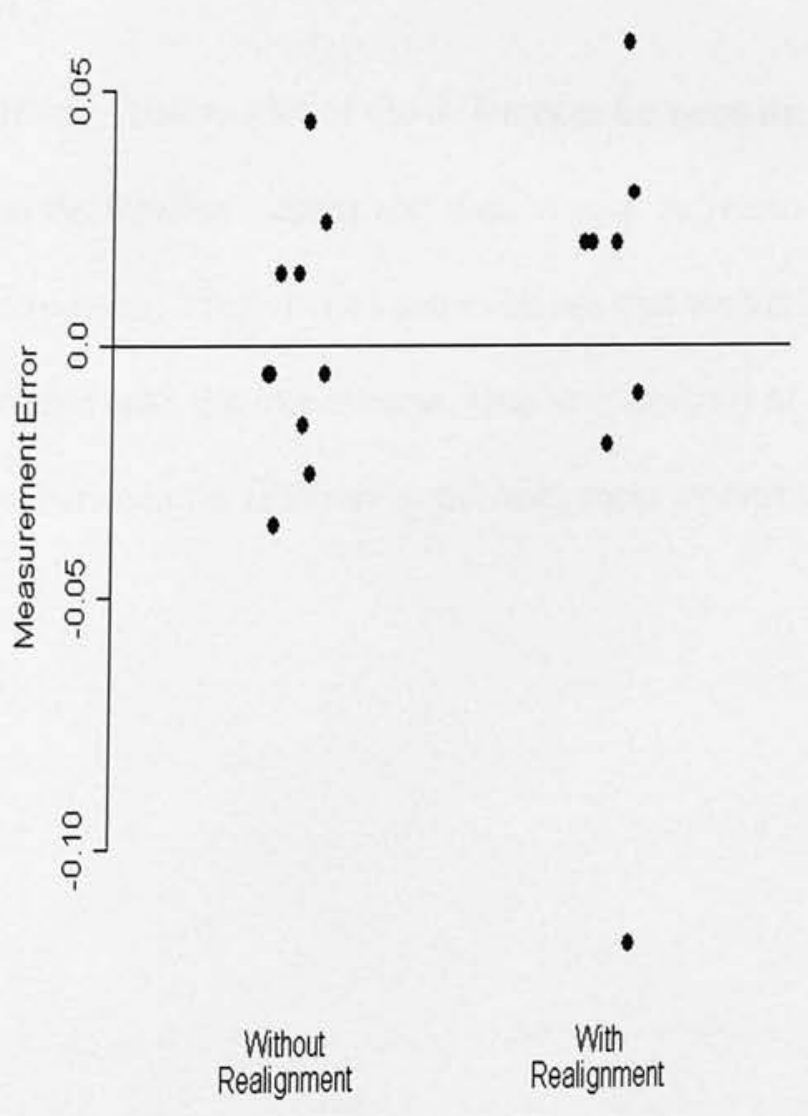


Variance $\left(\mathrm{mm}^{3}\right)$

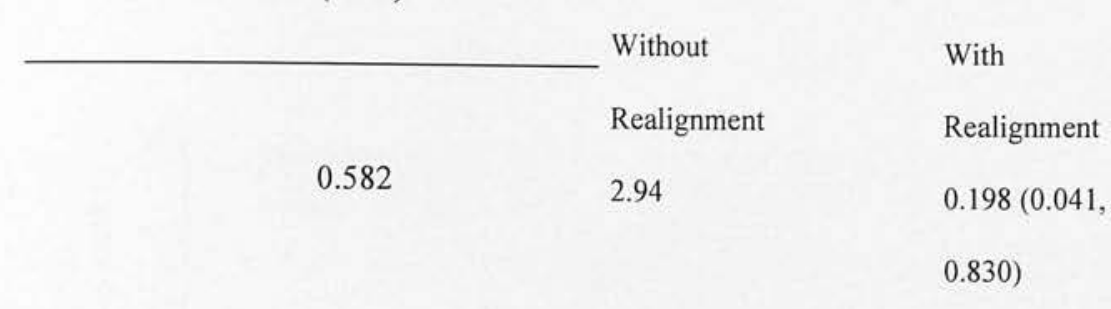

\begin{tabular}{|c|c|c|c|c|}
\hline All Data & \multirow[b]{2}{*}{0.582} & \multirow[b]{2}{*}{0.690} & \multirow[b]{2}{*}{$\begin{array}{l}0.843(0.153, \\
3.64)\end{array}$} & \multirow{2}{*}{$\begin{array}{l}0.028 \\
0.79\end{array}$} \\
\hline $\begin{array}{l}\text { Excluding } \\
\text { Outlier }\end{array}$ & & & & \\
\hline \multicolumn{5}{|c|}{$\begin{array}{l}\text { Table 3.1: Estimated variances of measurements made with or without realignment of } \\
\text { images, their ratio, with } 95 \% \mathrm{CI} \text {, and p-value from F-test of equality of variances }\end{array}$} \\
\hline
\end{tabular}

\subsubsection{Experiment 3}

Figure 3.7 shows the Bland-Altman plot of the differences between the first and second measurements made on the forehead, cheek and nose of each individual, versus the mean of the two measurements. There is no clear evidence that the variability of measurements is associated with the true volume. One-way analysis of variance revealed no association between the differences between measurements and facial region $(\mathrm{p}=0.34)$ 


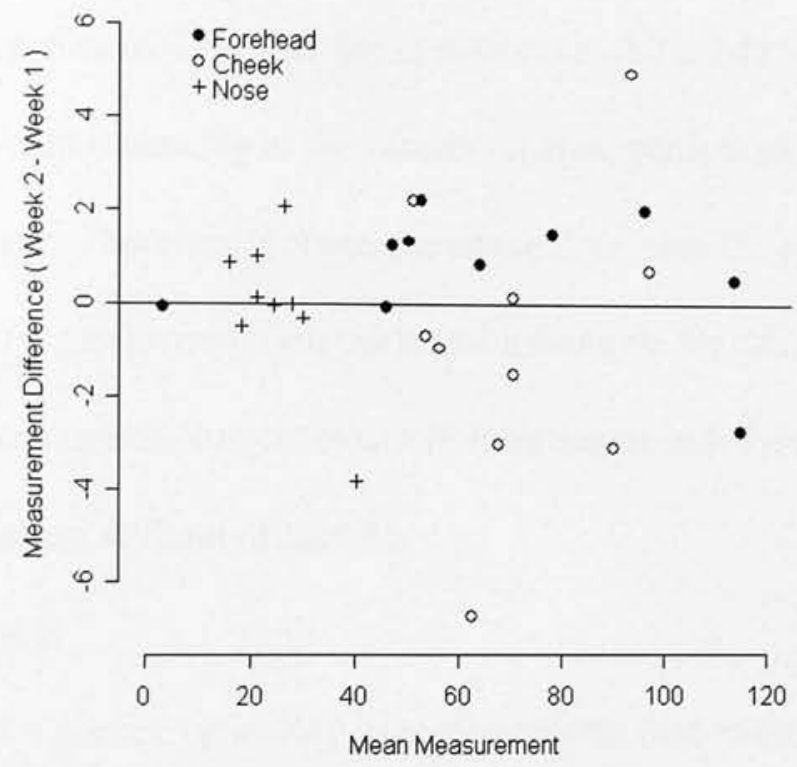

Figure 3.7. Bland-Altman plot for repeated measurements of forehead, cheek and nose of ten individuals, taken one week apart.

Applying variance components models to measure the test-retest reliability of the measurement method found the reliability for measurements of the forehead to be $99.9 \%$, for the cheek to be $98.3 \%$ and for the nose, $97.7 \%$.

\subsubsection{Discussion and Conclusions}

\subsubsection{Experiment 1}

Experiment 1 showed the measurement method to be generally consistent and accurate, with the exception of measurements made of the male face, which showed a slight underestimation of true volumes. The most likely explanation for this is that the silicone moulds created from the female face, although flexible, did not adhere to the exact contour of the male face despite adhesive glue. Importantly, the variability in measurement did not appear to be associated with true volume, indicating the technique to be consistent, or equally accurate at measuring large as small changes in facial 
volume. Attempts to measure 3-D volume change in the region of the chin following application of the chin mould were unsuccessful and therefore the chin mould was excluded from the experiment. This was due to distortion of the 3-D image on the inferior aspect of the chin extending to the submental area, particularly in the midline where the images merge. Therefore, volume measurements from the periphery of the 3D image e.g. chin, ears may prove problematic and inaccurate for this reason and repeated attempts to capture the subject from different angles may be required to capture the area of interest without distortion.

\subsubsection{Experiment 2}

Experiment 2 showed a greater variability in measurements made with realignment of images than without, though this finding is called into question when reanalysed with the exclusion of one outlying observation within the realigned measurements. Therefore no conclusive inferences can be drawn.

\subsubsection{Experiment 3}

Experiment 3 demonstrated measurements taken one week apart to be highly reliable, supporting the general conclusion that the method provides consistently accurate measurements of facial volume, which does not appear to vary by facial region or the overall volume of the facial feature being assessed.

\subsection{Results}

\subsubsection{Data completion}

Table 3.2 summarizes the numbers of patients with data collected during the follow-up period of the study. Patient recruitment was greatest to the Newfill group. However, this group also comprised the largest number of drop-outs. 
Table 3.2. Number of patients treated and follow-up 3-D data completed

\begin{tabular}{|c|c|c|c|c|}
\hline \multirow{2}{*}{ Treatment } & \multirow{2}{*}{$\begin{array}{l}\text { Patients } \\
\text { (N) }\end{array}$} & \multicolumn{3}{|c|}{ 3-D Volume Data } \\
\hline & & $2 \mathrm{mo}$ & $6 \mathrm{mo}$ & $12 \mathrm{mo}$ \\
\hline $\begin{array}{l}\text { Bio- } \\
\text { alcamid }\end{array}$ & 16 & 15 & 8 & $\begin{array}{c}7 \\
(43 \%)\end{array}$ \\
\hline Newfill & 20 & 14 & 7 & $\begin{array}{c}5 \\
(25 \%)\end{array}$ \\
\hline AFT & 12 & 7 & 7 & $\begin{array}{c}5 \\
(42 \%)\end{array}$ \\
\hline
\end{tabular}

A missing-value analysis comparing baseline volume measurements between those patients with complete data and those patients with incomplete data demonstrated no significant differences between the two groups. (Table 3.3) 
Table 3.3. Injected Volumes (Bio-Alcamid and AFT groups only), comparing those with or without follow-up volume measurements. Summaries shown are number of observations (number of missing values), mean (SD); p-values from two-sample t-tests

\begin{tabular}{|c|c|c|c|c|}
\hline \multirow{2}{*}{$\begin{array}{l}\text { Baseline } \\
\text { Measurement }\end{array}$} & \multirow{2}{*}{$\begin{array}{c}\text { Follow- } \\
\text { up } \\
\text { Visit }\end{array}$} & \multicolumn{2}{|c|}{$\begin{array}{c}\text { Follow-up Volume } \\
\text { Measurement }\end{array}$} & \multirow[t]{2}{*}{ p-value } \\
\hline & & Present & Absent & \\
\hline \multirow{3}{*}{$\begin{array}{l}\text { Injected } \\
\text { Volume } \\
\text { (Bio-alcamid } \\
\text { and } \\
\text { AFT groups } \\
\text { only) }\end{array}$} & $\begin{array}{c}\text { Month } \\
2\end{array}$ & $\begin{array}{c}21(1) \\
25.64 \\
(12.88)\end{array}$ & $\begin{array}{c}7(4) \\
17.29 \\
(7.30)\end{array}$ & $\mathrm{p}=0.118$ \\
\hline & $\begin{array}{c}\text { Month } \\
6\end{array}$ & $\begin{array}{c}15(0) \\
22.53 \\
(13.29)\end{array}$ & $\begin{array}{c}13(5) \\
24.73 \\
(11.18 \\
)\end{array}$ & $\mathrm{p}=0.643$ \\
\hline & $\begin{array}{c}\text { Month } \\
12\end{array}$ & $\begin{array}{c}12(0) \\
22.08 \\
(12.03)\end{array}$ & $\begin{array}{c}16(5) \\
24.66 \\
(12.57 \\
)\end{array}$ & $\mathrm{p}=0.590$ \\
\hline
\end{tabular}

Table 3.4 shows the number of patients who underwent revision procedures or retreatments during the trial follow-up interval.

Table 3.4 Number of revision procedures or re-treatments performed

\begin{tabular}{|c|c|}
\hline Treatment & Patients \\
& $(\mathrm{N})$ \\
\hline Bio- & 6 \\
alcamid & 3 \\
revision & \\
Major & \\
\hline revision & \\
\hline Fat & \\
\hline
\end{tabular}


Six patients in the Bio-alcamid group had a minor revision procedure (removal or reinjection of $1 \mathrm{cc}$ or less per cheek). In this group, 2 patients underwent a minor revision 12 months following the original treatment. Therefore follow-up data was completed according to the trial protocol prior to revision. One patient who underwent a minor revision 2 months following treatment moved away and was lost to further follow-up. In the remaining 3 patients, minor revision procedures were performed 6 months following initial treatment. Given the difficulty in accurately measuring small volumes of Bio-alcamid removed intra-operatively, the residual injected volume of Bio-alcamid was uncertain. Therefore, 12 month 3-D images were not completed in these patients. Only psychological data was completed at the defined follow-up intervals. Three patients in the Bio-alcamid group underwent major revisions with volumes injected ranging between 5-14cc per cheek. In these patients, the total injected volumes were re-calculated and further follow-up 3-D volume measurements were compared with the revised volumes. Follow-up psychological data was recommenced at intervals of 2, 6 and 12 months.

One patient in the fat transfer group with severe lipoatrophy underwent re-treatment during the trial follow-up period. A total of $32 \mathrm{cc}$ of fat was injected thus the follow-up data was completed as for a major Bio-alcamid revision: further 3-D volume measurements were compared with the revised injected volumes and psychological data was completed the defined follow-up intervals.

Two patients in the fat transfer group completed 12 months follow-up but re-entered the trial for Newfill therapy. In these 2 patients, baseline 3-D and psychological data was recorded prior to re-entry in the Newfill group and a second follow-up period was completed.

\subsubsection{3-D volume measurement}


The change in facial volume compared to baseline for each treatment group is summarised in Table 3.5 and Figure 3.8.

Additional statistical analysis of the dataset was conducted to investigate the effect of the high drop out rate and resultant small sample size at follow-up. Linear mixed effects regression (i.e. repeated measures ANOVA) was used to model all volume measurements over time. The model included random intercepts for each patient, fixed categorical effects of treatment and visit, and a general covariance structure. To estimate mean values by treatment and visit, a treatment-by-visit interaction was included. To assess the evidence for between-group differences in mean volumes, or mean changes in volume, at each visit, Wald tests were applied.

The results showed little difference when compared with the raw dataset and therefore only the raw data is presented for simplicity. A summary of the additional tests described are provided in Appendix 3 for reference only.

Table 3.5. Mean, SD and number of observations of volume injected or volume relative to baselineby visit and treatment type. Mean, SD and number of observations of changes over baseline, with paired t-test p-values for test of zero change.

\begin{tabular}{|c|c|c|c|c|c|c|c|c|c|}
\hline & \multicolumn{3}{|c|}{ Bio-Alcamid } & \multicolumn{3}{|c|}{ Fat } & \multicolumn{3}{|c|}{ NewFill } \\
\hline & Raw Data & $\begin{array}{c}\text { Change over } \\
\text { baseline }\end{array}$ & $\begin{array}{l}p \text {-value } \\
\text { for change }\end{array}$ & Raw Data & $\begin{array}{c}\text { Change over } \\
\text { baseline }\end{array}$ & $\begin{array}{l}p \text {-value } \\
\text { for change }\end{array}$ & Raw Data & $\begin{array}{c}\text { Change over } \\
\text { baseline }\end{array}$ & $\begin{array}{l}\text { p-value } \\
\text { for } \\
\text { change }\end{array}$ \\
\hline cted & $25.5(13.4), \mathrm{N}=18$ & & & $20.1(9.3), N=10$ & & & $-(-), N=0$ & & \\
\hline lonth & $28.3(13.9), N=15$ & $1.1(6.7), N=15$ & $\mathrm{p}=0.083$ & $17.1(7.8), N=7$ & $-4.9(5.9), N=6$ & $\mathrm{p}=0.156$ & $8.7(7.2), N=14$ & $-(-), N=0$ & $\mathrm{p}<0.001$ \\
\hline lonth & $26.7(15.5), N=8$ & $0.7(3.1), N=8$ & $p=0.742$ & $11.2(5.2), \mathrm{N}=7$ & $-7.3(7.1), N=7$ & $\mathrm{p}=0.047$ & $12.6(10.7), \mathrm{N}=7$ & $-(-), N=0$ & 0.016 \\
\hline Month & $27.7(13.4), N=7$ & $3.4(2.6), N=7$ & $p=0.031$ & $10.0(7.9), \mathrm{N}=5$ & $-9.0(9.5), N=5$ & $\mathrm{p}=0.312$ & $12.3(13.5), \mathrm{N}=5$ & $-(-), N=0$ & 0.019 \\
\hline
\end{tabular}


Figure 3.8. Change in measured 3-D facial volume for each treatment group

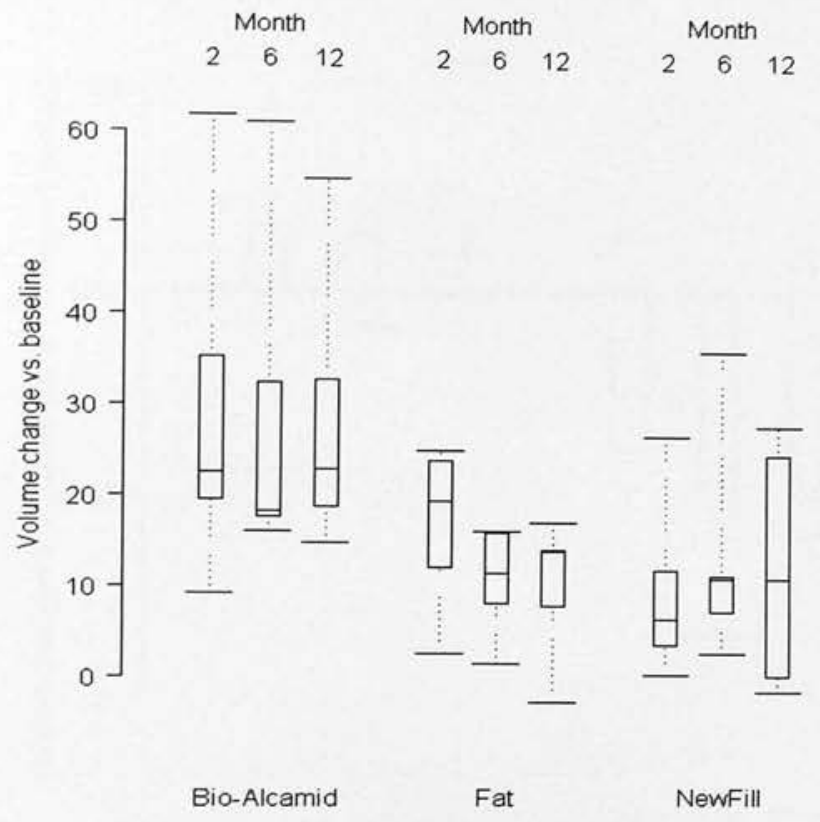

The greatest mean injected volume was seen with Bio-alcamid $(25.5 \mathrm{cc})$. A paired t-test demonstrated no significant difference in mean volume change relative to zero except at 12 months.

The mean injected volume of autologous fat was $20.1 \mathrm{cc}$. Although no difference in mean volume change relative to baseline was demonstrated at 2 months, a reduction in mean volume to $11.2 \mathrm{cc}$ at 6 months $(\mathrm{p}=0.03)$ and $10 \mathrm{cc}$ at 12 months following treatment was seen $(p=0.1)$. This represents $66 \%$ of injected volume relative to baseline remaining at 6 months and 54\% at 12 months (Figure 3.9). 
Figure 3.9. Percentage of injected volume remaining at follow-up

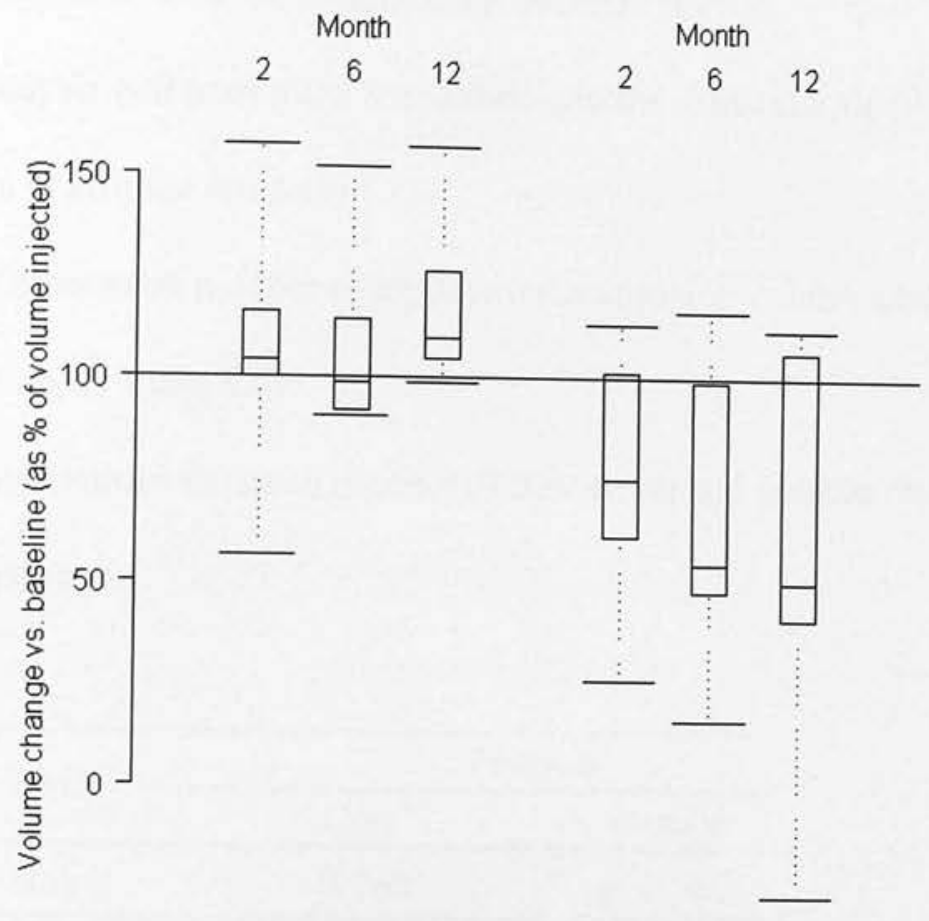

Bio-Alcamid

Fat

\begin{tabular}{|l|l|l|}
\hline & Bio-alcamid & Fat \\
\hline 2 Month & & \\
Mean (SD) & $107.3(21.8$ & $75.4(31.1)$ \\
Median (IQR) & $104.5(100.2$, & $75.3(64.7,95.0)$ \\
[MIN, MAX] & $112.7)$ & {$[25.5,113.5]$} \\
& {$[56.4,157.4]$} & \\
\hline 6 Month & $106.7(21.2)$ & $66.4(34.9)$ \\
Mean (SD) & $99.1(92.1,109.8)$ & $54.1(48.3,90.9)$ \\
Median (IQR) & {$[90.9,151.7]$} & {$[15.3,116.7]$} \\
[MIN, MAX] & & \\
& & \\
\hline 12 Month & $117.0(19.3)$ & $56.2(57.3)$ \\
Mean (SD) & $109.6(106.9$, & $49.8(40.7,106.7)$ \\
Median (IQR) & $120.1)$ & \\
[MIN, MAX] & {$[99.0,156.5]$} & \\
& & \\
\hline
\end{tabular}

The mean measured 3-D volume change for Newfill was $8.6 \mathrm{cc}$ at 2 months, $12.5 \mathrm{cc}$ at 6 months and $12.2 \mathrm{cc}$ at 12 months. The t test (scores at baseline are all equal to 0 ) 
comparing all follow-up intervals compared to baseline shows that after treatment the mean cheek volumes were all significantly different from 0 .

Statistical analysis comparing the 3 treatment groups showed a significant difference in mean volume at all time intervals.

No correlation between number of treatment sessions and volume change over time was demonstrated (Table 3.6)

Table 3.6. Correlations between number of treatments and volume changes over time for NewFill patients.

\begin{tabular}{ccc}
\hline \multirow{2}{*}{ Visit } & \multicolumn{2}{c}{ Pearson } \\
\cline { 2 - 3 } & Corr $^{\mathrm{n}}$ & $\mathrm{p}$-value \\
\hline Month 2 & 0.263 & $\mathrm{p}=0.363$ \\
\hline Month 6 & 0.115 & $\mathrm{p}=0.806$ \\
\hline Month 12 & 0.059 & $\mathrm{p}=0.924$
\end{tabular}

\subsection{Discussion}

\subsubsection{Data completion}

Forty-eight patients received treatment for facial lipoatrophy but only 17 patients $(35 \%)$ had complete12 month 3-D volume data. The number of drop-outs was greatest in the Newfill group with only $25 \%$ of patients completing follow-up. The fat group comprised the smallest number of recruited patients and complete follow-up data was also poor (42\%). Complete data in the Bio-alcamid group was $43 \%$. The large number of drop-outs in this study was thus very disappointing but highlights the difficulties of clinically-based research which relies on patient motivation. From the outset of the study, it was evident that comparison between groups with respect to treatment outcomes would be subject to bias due to the non-randomised design of the study and 
the small numbers in each group. Thus the study design was primarily observational. However, the declining number of patients at follow-up further confounded the difficulties of interpreting results and drawing conclusions within and between groups. An attempt to investigate and potentially compensate for drop-outs using regression methods was conducted at the outset of data analysis. There were few differences in results when compared to the raw data and therefore the more complex statistical analysis was not felt to provide a beneficial contribution to the results. Only the raw data was used for interpretation of results to provide greater clarity and to avoid unnecessary distortions in the data. Statistical analysis of results was performed using parametric tests. As an additional measure, equivalent non-paramentric tests were applied to all results to investigate any potential differences from assuming a normal distribution. Comparison of results using parametric and non-parametric test demonstrated no difference. Therefore, only the parametric results were presented in this study on the basis that parametric tests are, in general, more powerful and should be used if at all possible.

There are various factors that may account for the high drop-out rate for 3-D imaging. Firstly, patient motivation to complete follow-up after treatment declined, particularly by 12 months. Secondly, some patients experienced commuting difficulties as the 3-D system was based in Livingston, approximately 20 miles from where most patients lived in Edinburgh. Several patients were reluctant to take time off work, which was mainly related to commuting time. This was an even greater obstacle for patients recruited from Glasgow (approximately 30 miles from Livingston). In an attempt to overcome this practical issue, the DI3D system was transported to an HIV clinic in Glasgow on several occasions but unfortunately many patients still failed to attend when invited for follow-up. A large proportion of the Newfill group were recruited from Glasgow and this accounts for the particularly poor drop-out rate in this group. 
Thirdly, technical issues with the 3-D system also contributed to incomplete data capture. No images could be obtained during one of the capture sessions due to a faulty camera. Several patients who had attended for clinical follow-up on that occasion did not attend the re-arranged session and were thus lost to follow-up.

The problem of analysis and interpretation of results therefore requires some reflection. Would the results have been different if the data was more complete? Statistical analysis of baseline volume and psychological measurements, comparing those patients with and without follow-up data, demonstrated no significant difference. However, it is likely that those patients who did attend for review had motivation to do so, thus biasing the results. In many cases, patients attended for review to discuss a complication or concern over the aesthetic result following treatment. Conversely, it is likely that those patients who did not attend for review were satisfied with the outcome of treatment. Indeed, this appears to be the case for many of the drop-outs in the Newfill Glasgow group following communication with the health professionals involved in their ongoing HIV management. Frustratingly, the quantification of possible facial volume maintenance and improved psychological outcome is lacking in the results. It is possible that a greater mean volume of fat remaining at 6 and 12 months would have been demonstrated in the complete data set. Also, reliability of the12-month volume data for Newfill therapy would have been more robust. This would have been especially useful to demonstrate the delayed volume augmentation mechanism of action and duration of effect.

A further difficulty with the analysis and interpretation of results was the problem of revision procedures during the follow-up period, particularly in the Bio-alcamid group. Revisions in the Bio-alcamid group were subdivided into minor (1cc or less removed or re-injected per cheek) or major if larger volumes were re-injected. Although this alteration to the original method is not ideal, the total volume injected could be 
recalculated for the 3 patients who had a major revision and subsequent volume measurements were compared with the revised volumes. The main difficulty arose with analysis of follow-up 3-D data in the minor revision group. Unfortunately, the volume of product removed is difficult to measure intraoperatively. Therefore, subsequent 3-D measured volumes could not be compared with an unknown remaining injected volume. The dilemma of how to proceed with 3-D data follow-up was applicable to 3 patients in this group. It was decided that subsequent 3-D measurements would be unreliable and therefore inconclusive. This led to incomplete 12 month data in these patients and difficulty with subsequent statistical analysis and interpretation. For example, the raw data shows similar mean volume measurements at all follow-up intervals compared to injected volumes. However, the t-test for change showed significance at 12 months which is mostly likely due to the smaller sample size and revision procedures performed. All of these patients completed the psychological data at the defined trial follow-up intervals and the interpretation of these outcomes is discussed in chapter. Although two patients in the fat group switched to the Newfill group, 12 month follow-up data was completed prior to the change.

\subsubsection{3-D Facial volume results}

For patients with severe facial lipoatrophy, Bio-alcamid $\circledast$ has been advocated as an effective treatment option. In this study, patients in the Bio-alcamid group had the most severe clinical grade of lipoatrophy and required the greatest injection volumes to achieve correction. Therefore, one advantage of Bio-alcamid in reconstructive surgery is that it can be injected in large quantities to achieve an immediate correction. The product is also considered to be a permanent filler due to the stability of its chemical properties and resistance to hydrolysis (Ramires, Miccoli et al. 2005). Indeed, the results of this study, utilising 3-D imaging as a sensitive measure of facial soft tissue 
volume change, demonstrated no significant difference in measured volume postoperatively compared to injected volumes. The distribution of volume change in the treated areas was not analysed in this study but would be an extremely useful investigation of product migration in future.

The use of autologous fat transfer has also been described in the treatment of HIVlipodsytrophy but the outcome of the procedure is often based on subjective measures such as clinical assessment or photographs. One recent study has utilised ultrasound to measure dermal and subcutaneous thickness following treatment with fat transfer (Guaraldi, Orlando et al. 2005). However, measurements were obtained from only one area within the cheek and are operator-dependant. This study provides new, objective data on 3-D measurements of transferred fat in patients with HIV lipodystrophy. The results showed no difference in mean volume of transferred fat compared to baseline at 2 months. Despite a reduction in mean measured 3-D volume of 7.3cc at 6 months and $9 \mathrm{cc}$ at 12 months, a significant difference compared to baseline was shown at 6 months only. Percentage of volume remaining at follow-up compared to injected volume was $66 \%$ at 6 months and $56 \%$ at 12 months. The pattern of volume change observed was highly variable between patients: some patients retained the injected volume throughout follow-up; some patients demonstrated minimal or no change in volume; and some patients had a gradual reduction in volume post-operatively. It is possible that the variability in results reflects the underlying lipodystrophic process. Indeed, one patient who underwent a combined procedure of autologous fat transfer and liposuction to the DCFP developed DCFP recurrence coinciding with complete resorption of the transferred fat. This suggests that lipodystrophy may be contributing to the success of transferred fat in this group of patients. Thus, patients with HIV lipodystrophy should be advised about the unpredictable outcome of this procedure. Also, the results of fat transfer in this study are very specific to HIV lipodystrophy patients and cannot be 
applied to fat transfer in non-HIV patients. There were no reported changes in HAART therapy, haematological parameters, or weight during follow-up although one limitation of the study is that these variables were not formally measured. Another important factor to consider when analysing the results of fat transfer is that 3-D measurements only represent a change in volume. The composition of the volume change is unknown. Therefore, the remaining volume change could either represent survival of the transferred fat or simply fibrosis. There is emerging evidence for the role of stem cells in transferred fat and this is an interesting area for further investigation.

Newfill therapy is well-established treatment for HIV-associated facial lipoatrophy and previous studies have demonstrated increased ultrasound measurements of dermal thickness and improved quality of life up to 96 weeks. However, this study provides new information on 3-D soft tissue volume changes following Newfill therapy. The results show a mean change in volume of $8.7 \mathrm{cc}$ at 2 months, which increased to $12.6 \mathrm{cc}$ at 6 months and was maintained at 12 months $(12.3 \mathrm{cc})$. Only one paper has been published since commencement of the trial which provides 3-D volume data on Newfill therapy for HIV lipodystrophy. In this study, 100 patients with HIV lipodystrophy were treated with a mean of 4.85 treatment sessions per patient and follow-up extended to 24 months (Ong, Clarke et al. 2009). The mean volume change was significantly different to baseline at all time points and the mean difference per cheek was $2.81 \mathrm{cc}$. The mean volume changes in Ong's study were less than the results seen in this trial. However, there were some differences in method which could explain these findings. Patients were restricted to 4 or 5 treatment sessions on Ong's study, and injections were carried out to the cheeks only. In this study, treatment sessions with Newfill were carried out until both the patient and practitioner were satisfied that restoration of facial volume had been achieved and a wider area extending to the temples was treated. Thus, the majority of patients received 7 or more treatment sessions. It is interesting to evaluate 
the relationship between volume augmentation and number of treatment sessions. This study demonstrated no correlation between volume change over time and number of treatment sessions. However, limitations include small sample size and lack of complete follow-up data. Therefore, it is difficult to draw conclusions. From the volume data available and clinical impression of patients and practitioners, Newfill appears to be an effective treatment for patients with mild to moderate facial lipoatrophy but may not provide sufficient volume augmentation for patients with severe lipoatrophy. This is related to the mechanism of action of Newfill which stimulates neocollagenesis within the dermis. Patients in this study also had a variability in mean volume change achieved, unrelated to number of treatment sessions. This raises some questions regarding the maximum volume change that can be achieved with Newfill and whether this is related to number of treatment sessions or individual response to neocollagenesis. This would be a very useful area of further investigation and would be helpful in decision-making about choice of treatment in patients with severe lipodystrophy.

\subsection{Conclusions}

Based on literature review, this study remains the first to provide objective, 3-D facial volume measurements following 3 different treatments for HIV facial lipoatrophy. Comparison between treatment groups, in conjunction with additional outcome measures are presented in the overall discussion and conclusions of this thesis. In addition, experiments to validate the DI3D system and software have demonstrated the accuracy and reproducibility of the system to measure 3-D facial volume. 


\section{Chapter 4. Evaluation of psychological morbidity in HIV lipodystrophy}

\subsection{Aims}

1. To evaluate change change in body image assessment, anxiety and depression following treatment for HIV-associated facial lipoatrophy.

2. To compare psychological characteristics between HIV seropositive patients with and without lipodystrophy and HIV seronegative men who have sex with men (MSM).

3. To investigate the correlation between psychological distress, clinical grade of facial lipoatrophy and facial volume change following treatment for HIV associated facial lipoatrophy.

\subsection{Method}

\subsubsection{Design}

A prospective, follow-up study was designed to assess change in psychological morbidity in a cohort of patients undergoing 3 different treatments for HIV lipodystrophy (autologous fat, Newfill and Bio-alcamid). In addition, a case-control study with two control groups was designed to investigate variables that may influence psychological distress in patients with HIV-associated lipodystrophy. (Figure 4.1) 
Figure 4.1. Flow diagram of Study Design

\section{Case-control Study}

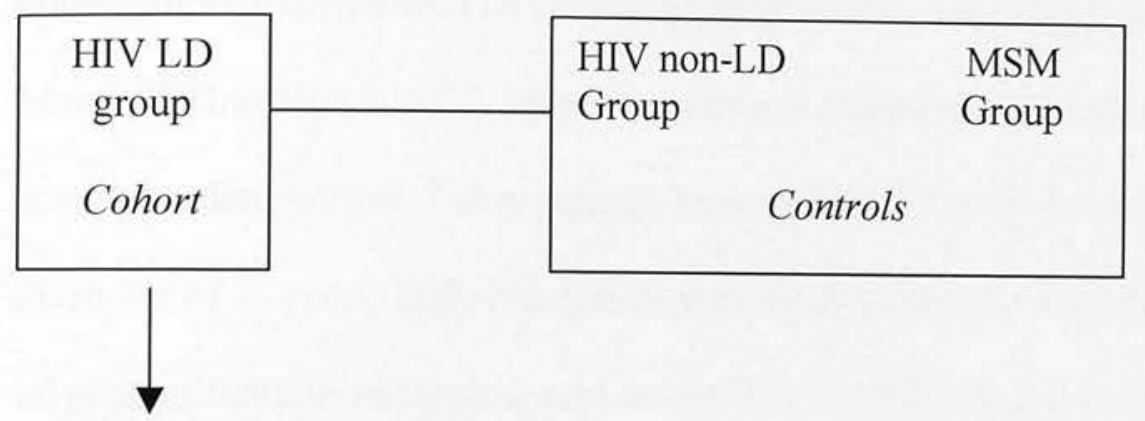

Prospective observational study

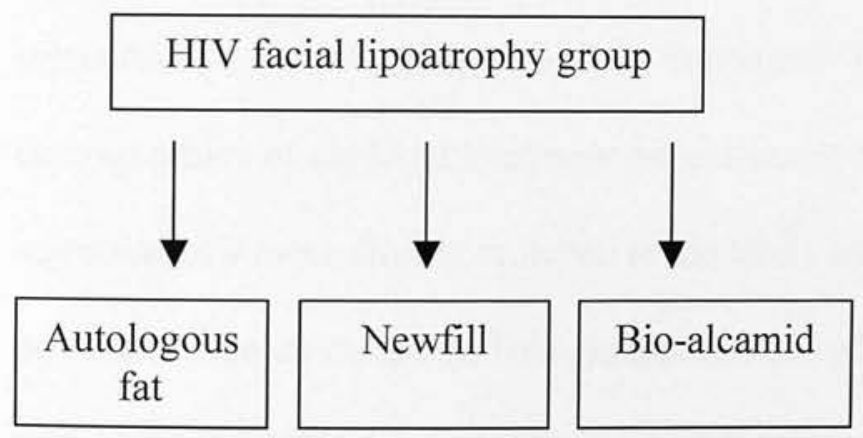

\subsubsection{Control groups}

\subsubsection{Selection of groups}

When considering psychological distress in the cohort group, and particularly the impact of a surgical intervention, it is important to identify potential confounding variables. To avoid systematic bias in the case control study, the aim should be for the groups being compared to be as like one another as possible except for the particular difference being examined. Psychological distress associated with development of HIV lipodystrophy is the condition under investigation in this study. However, the impact of living with HIV infection was considered an important factor contributing to psychological wellbeing. A control group comprising HIV patients without lipodystrophy was therefore selected to investigate the effect of lipodystrophy alone. In order to evaluate the effect of HIV infection to positively or negatively influence body image perception, an additional control group of non-HIV patients was required. 
It is also important to consider the influence of factors such as age, sex, and ethnic origin on body image assessment. Demographic data was collected on the local population of patients with HIV lipodystrophy treated within the Edinburgh Unit from March 2003 to March 2006. Thirty-two patients treated were identified using the hospital coding system. Thirty patients were male and 2 patients were female with a mean age of 46 years. Only one patient was not of Caucasian origin and although not all patients volunteered their sexual orientation, it was estimated that approximately 80 $90 \%$ of patients were MSM (men who have sex with men). Consideration was given to selection of a control group of healthy volunteers. However, following reflection on the demographics of the local treatment population, it seemed appropriate to select a control group more closely matched to the likely treatment group with regards to sex, age, and sexual orientation. Thus, in anticipation of a similar pattern of referrals to the trial, a second control group of men who have sex with men (MSM) was selected to evaluate psychological morbidity in this subgroup of the male population.

\subsubsection{Psychological measures}

\subsubsection{Selection of tools for the study}

The choice of measurement tools selected for the study was based on review of the literature and consultation with professionals in psychiatry and psychology.

Assessment of outcomes in aesthetic surgery is extremely important, as patient satisfaction is the predominant factor in determining success. Although various scales have been used to assess outcomes, none has achieved widespread use (Ching, Thoma et al. 2003). To date, assessment of outcome from aesthetic surgery has involved subjective assessment by the patient and surgeon, quality of life outcomes and psychological assessments of psychiatric disorders, self-esteem and body image. In one review of 43 papers, an attempt was made to identify appropriate instruments to assess 
outcome in aesthetic surgery (Ching, Thoma et al. 2003). The authors discovered that assessment tools fell into four categories: satisfaction, objective, psychological and quality of life assessments. Each of the instruments was reviewed to evaluate practicality, validity, reliability and sensitivity to change. The authors concluded that quality-of-life and body-image measures are of greatest value in determining aesthetic surgery outcomes. The Derriford Appearance scale was identified as an ideal scale for use in aesthetic surgery.

The DAS 24 was selected as an outcome measure for the study as it was designed specifically to measure psychological distress and dysfunction characteristic of disfigurements, distress and aesthetic problems of appearance. It is highly sensitive as a measure of change following treatment and for evaluating the merits of one treatment protocol against another (Harris and Carr 2001). It is psychometrically robust and discriminates well between patient groups, between clinical and non-clinical populations, and within the general population, between those concerned and not concerned about their appearance (Carr, Moss et al. 2005).

\subsubsection{Derriford Appearance Scale}

The Derriford Appearance Scale (Appendix 4) is a condition-specific quality-of-life measure developed for assessing concern about physical appearance. It seeks to measure adjustment to problems of appearance, which the authors define as negative emotions of fear, social anxiety, shame, and negative affect along with a behavioral response of avoidance and withdrawal that frequently disrupts lifestyle. Initially designed as an experimental scale (DAS 136) composed of 136 items, this was refined to two user-friendly versions: the DAS 59 composed of 59 items (Harris and Carr 2001) and the DAS 24 with 24 items. (Carr, Moss et al. 2005) In designing the DAS 24, items were selected from the DAS 136 to include as broad a range of behavioural and emotional responses to being visibly different as possible. 
The first page of the DAS 24 collects demographic information and identifies the body part(s) about which the respondent may be self-conscious. Then in a series of 24 items, respondents are asked to choose from a range of responses. Response categories of 'extremely' to 'not at all' are used when items relate to an emotional response (e.g. 'How distressed do you get when you see yourself in the mirror'). An 'almost always' to 'never/almost never' response category is used for items relating to particular behaviours (e.g. 'I avoid going out of the house'). For items that include a not applicable (N/A) option, N/A always scores zero. The remaining response categories score one to four and the response category indicating more distress scores the highest. Items one to twenty four inclusive are summed to provide the total score. The minimum score is 11 and the maximum is 96 . There is no threshold score to indicate 'caseness' as the authors of the scale believe that adjustment to appearance concerns is a continuum. The DAS 24 has been administered to clinical and non-clinical populations.(Harris and Carr 2001) Data was collected from 535 participants in a multi-centre trial. Around half were recruited from plastic surgery clinics and the remainder from waiting lists. The DAS 24 was sent by post to a non-clinical population and 1170 responses were received. The results were compiled to provide norm tables. To evaluate validity of the DAS 24 , five additional scales to measure psychological outcome were completed by participants. The internal consistency of the scale was high (Cronbach alpha $=0.92)$. The reliability of the scale, indicated by test-retest correlation, was good $(r=0.82-0.88)$. Concurrent validity, measured by comparison with a range of established scales, revealed a good relationship with the DAS 59 (Pearson correlation 0.88) and moderate positive correlation with subscales of the Crown Crisp Experimental Inventory (Pearson correlation $0.5-0.66$ ).

To date, there are no published studies to evaluate the DAS-24 in a cohort of patients with HIV lipodystrophy. 
Research indicates that individuals with disfigured appearance are prone to depression, anxiety, shame and interpersonal difficulties. Newell and Marks suggested that the difficulties experienced by those with facial disfigurements might be due to phobic anxiety specific to social situations. Indeed, research has shown that people with facial disfigurements exhibit similar anxiety and depression scores to individuals with agoraphobia and social phobia (Tebble, Thomas et al. 2004). Thus a 'fear avoidance' model has been proposed and suggests that the fear of response of others perpetuates social anxiety and the subsequent avoidance of social interactions. In a study by Sen et al, symptoms of anxiety or depression were reported in over $30 \%$ of patients with severe maxillo-facial trauma (Sen, Ross et al. 2001). In particular, symptoms of anxiety and depression have been reported in patients with HIV lipodystrophy, although quantification utilizing validated methods is lacking.

A measure of anxiety and depression was therefore considered to be an important outcome for the study and the Hospital Anxiety and Depression Scale was selected on this basis.

\subsubsection{Hospital Anxiety and Depression Scale}

The Hospital Anxiety and Depression Scale (HADS) was developed in 1983 to detect states of depression and anxiety among patients in non-psychiatric hospital clinics (Zigmond, Snaith et al. 1983). It is divided into an anxiety subscale and depression subscale, each containing seven items (Appendix 5). Assessment of severity of anxiety and depression is rated on a four-point (0-3) scale. Scores of 0-7 in respective subscales are considered normal, with 8-10 borderline and 11 or over indicating clinical 'caseness'. The authors state that the scale may be a useful devise for assessing change in a patient's emotional state as well as for assessing the presence of clinically significant degrees of anxiety and depression. 
In a comprehensive review of the literature, the validity of the Hospital Anxiety and Depression Scale was assessed based on 747 studies which used the scale (Bjelland, Dahl et al. 2003). The internal consistency of the scale was found to be good in all of the reported studies. As a case finder for anxiety and depression, optimal sensitivity and specificity was achieved when caseness was defined by a score of 8 or above for HADS-A and HADS-D. The concurrent validity of HADS was also found to be good. The authors conclude that HADS performs well in screening for the separate dimensions of anxiety and depression and 'caseness' of anxiety and depression in patients from non-psychiatric hospital clinics.

\subsubsection{Evaluation of selected tools in HIV lipodysotrophy patients}

Although both of the instruments chosen are well established and reliable, data to support their application to HIV lipodystorphy is lacking. In order to investigate whether the selected tools address the psychological issues associated with HIV lipodystrophy, a series of informal, semi-structured interviews were designed. A sample of 7 patients with HIV lipodystrophy treated within the Edinburgh Unit was selected. A set of reference interview questions was devised to prompt discussion (Appendix 6) but most patients volunteered information about their individual experiences. Finally, patients were asked to complete the psychological scales selected for the study and provide feedback.

The qualitative interviews raised several psychological issues, some which were common to all patients and others experienced only by individuals. The stigmatization of HIV lipodystrophy was one of the most apparent issues. Patients felt that their HIV diagnosis was apparent from their appearance, particularly amongst the HIV and MSM communities where awareness is increased. This led to some perceived difficulties in social interactions and forming relationships. Patients were often misjudged because of their appearance and described public perception of facial wasting as a consequence of 
severe illness or intravenous drug abuse. Subsequently, patients felt members of the public treated them with trepidation. Most patients regarded their face as their most important physical feature. Although they were able to disguise other body parts with clothing, their face was always exposed. Many patients had negative feelings about the change in their appearance, and some felt depressed. Several patients admitted to concerns about appearance prior to the development of lipodystrophy and feel this reflects pressure from the MSM community which places emphasis on physical attractiveness. One patient was concerned that his gaunt appearance conjured similar negative images of HIV or AIDS portrayed in the media. For some patients who feel systemically well and have good HIV control, the morphological features of lipodystrophy are a visible reminder of their HIV status. Most patients felt selfconscious about their appearance, particularly in public places; however, for the majority it did not affect their work or social life. Some factors such as increasing age and a stable relationship appeared to influence concern over appearance in a positive way. Although most patients did not complain about physical symptoms related to lipodystrophy, one patient felt that peripheral wasting of his limbs caused weakness and shortness of breath on exertion.

Both psychological scales were acceptable to all of the patients questioned. Some patients provided useful feedback on some of the items in the DAS24. Overall, the selected psychological measures were considered appropriate to address the aims of the study in this patient group.

\subsubsection{Recruitment}

HIV-seropositive patients with lipodystrophy were recruited from referrals to plastic surgery clinics in Edinburgh and Glasgow. Participants in the control groups were recruited from Infectious Diseases and Genito-Urinary Medicine clinics. A medical 
history and lipodystrophy-specific examination using the patient and physician assessment of lipodystroph (Carr, Law et al. 2003) was carried out on all HIVseropositive patients by 2 doctors. This assessment records independently any lipoatrophy or fat accumulation in each of the face, neck, dorsocervical spine, arms breasts, abdomen, buttocks, and legs, as well as the presence of any lipomata. Other data collected included the patients' age, ethnic status, date of diagnosis, mode of HIV acquisition, current medication and treatment history, viral load/ CD4 count, and fasting glucose, cholesterol and triglycerides.

Patients were recruited to the trial if the eligibility criteria were satisfied. A patient information sheet was provided and informed consent was obtained.

\subsubsection{HIV-seropositive with lipodystrophy (HIV LD) group}

\section{Inclusion Criteria}

1. Patients over 18 years of age.

2. HIV seropositive.

3. Patient and physician agreed body changes attributable to lipodystrophy and amenable to surgical treatment.

4. Undetectable viral load and CD4 count $>200$ cells $/ \mathrm{mm}^{3}$

\section{Exclusion Criteria}

1. Pregnancy.

2. Inflammation/ infection of skin at site of treatment.

3. Concurrent illness/ medical problems precluding general anaesthetic.

4. Concurrent treatment with anti-coagulants

\subsubsection{HIV-seropositive without lipodystrophy (HIV non LD) group}

\section{Inclusion Criteria}

1 Age 18-60 years 
2 No morphological features of lipodystrophy as agreed by patient and physician

3 Time since HIV diagnosis $>6$ months

\section{Exclusion criteria}

1. Any known psychiatric disorder

\subsubsection{HIV-seronegative men who have sex with men (MSM) group}

\section{Inclusion Criteria:}

1 Age 18-60 years

2 MSM

3 HIV-seronegative test within past 6 months

Exclusion criteria:

1. Any known psychiatric disorder

\subsubsection{Data Collection}

\subsubsection{HIV LD Cohort}

Demographic data was collected including the patients' age and ethnic status. Data relating to the patients' HIV status was recorded including; date of diagnosis, mode of HIV acquisition, current medication and treatment history, viral load/ CD4 count. Information on the patients' past medical history, drug history and relevant social history were also collected.

\section{Clinical grading}

The HIV Outpatient Study (HOPS) scoring system (incorporated in the lipodystrophyspecific assessment utilized for patient recruitement) was used to rate the degree of lipoatrophy as absent $=0$, mild $=1$ (noticeable on close inspection), moderate $=2$ (readily noticeable by patient and physician) or severe $=3$ (readily noticeable to a casual observer) by both the patient and lead investigator. 
The DAS-24 and HADS were completed by all patients pre-operatively and repeat measures were obtained at 2, 6 and 12 months following completion of treatment.

\section{3-D imaging}

Measurement of 3-D facial volume change in HIV LD patients undergoing treatment for facial lipoatrophy was performed at 2, 6 and 12 months post-operatively. The details of this study are provided in chapter 3.

\subsubsection{Control groups}

Demographic data was recorded on the age, sex, ethnic origin, and sexual orientation of all participants. For the HIV non LD group, data was collected on the patients' HIV history including; date of diagnosis, mode of HIV acquisition, treatment history, and viral load/ CD4 count.

Psychological measures

All participants completed the DAS- 24 and HADS scales at the time of recruitment to the trial. No repeat measures were performed.

\subsubsection{Statistical analysis}

\subsubsection{Case control study}

Baseline patient demographics (age, treating unit, ethnicity) were summarised by study group (HIV LD, HIV non-LD and MSM), as a whole and by gender, where appropriate. Subgroups were compared by analysis of variance (ANOVA). For the two HIV groups, disease-related factors (main HIV risk factor, duration of HIV, duration of HAART, viral load, CD4 count and BMI) were summarised as a whole and by gender. Subgroups were compared by analysis of variance (ANOVA).

Baseline patient demographics (age, treating unit, ethnicity) were summarised by study group (HIV LD, HIV non-LD and MSM), as a whole and by gender, where 
appropriate. Subgroups were compared by analysis of variance (ANOVA). For the two HIV groups, disease-related factors (main HIV risk factor, duration of HIV, duration of HAART, viral load, CD4 count and BMI) were summarised as a whole and by gender. Subgroups were compared by analysis of variance (ANOVA). Baseline DAS-24 and HADS depression and anxiety (HADS-D and -A) scores were summarised by study subgroup. Mean scores were compared between study subgroups using analysis of variance (ANOVA). Mean scores were compared between study subgroups as a whole, and following exclusion of non-MSM and non-Caucasian individuals from each subgroup, using analysis of variance (ANOVA).

Comparative reference values were extracted from the published norm tables for the DAS-24 based on large multi-centre trials. Reference values were presented by gender for DAS-24 scores in a general population and in a clinical population (including subgroups with concerns relating to the head and neck region. The norm tables supply data on 3 different age ranges: 18 - 30 years, 31-60 years and $61+$ years. To approximate the age distribution of the study population, reference values were derived from individuals aged 31-60 years. Study subgroups were compared to the reference populations using two-sample t-tests.

\subsubsection{Prospective study of HIV LD treatment groups}

Patient demographics, HIV-related factors and clinical features were summarised by treatment group, and compared using analysis of variance (ANOVA) for continuous measures and Fishers exact test for categorical variables. 
To assess the potential for bias due to loss to follow-up, the baseline characteristics of those with or without follow-up volume measurements at 2, 6 and 12 months were summarized, and compared with two sample t-tests.

Psychological measures (DAS-24, HADS-A and HADS-D scores) at baseline and at 2, 6 and 12 months post-treatment, are summarized for all HIV LD patients undergoing treatment for facial lipoatrophy, as a whole and by treatment group. Changes over baseline were tested with paired t-tests, within treatment groups.

Correlations between changes in psychological measures over baseline and facial volumes at 2,6 and 12 months were tested using Pearsons correlation coefficient. Differences between psychological scores for DAS and HADS within patients subdivided according to clinical grade of lipoatrophy (assessed by both patient and physician) were tested using ANOVA. The percentage agreement of patient versus physician grading of severity was calculated and tested using McNemar's test.

\subsection{Results}

\subsubsection{HIV LD cohort versus control groups}

\subsubsection{Demographic data}

The DAS-24 and HADS were completed by 56 patients with HIV-associated lipodystrophy referred for plastic surgery, and similar numbers of HIV-seropositive patients without lipodystrophy and HIV-seronegative MSM volunteers (Table 4.1) Differences in baseline demographic data between groups are summarized as follows.

There was a significant difference in mean age between participants in the MSM and HIV LD group (10 years) and HIV non-LD group (6 years). A greater proportion of patients in the HIV LD group were recruited from Edinburgh $(80 \%)$ compared to a larger number of patients in the HIV non-LD group who were recruited from Glasgow 
(81\%). The majority of patients in both the HIV LD and MSM groups were Caucasian compared to a slightly more variable ethnic mix in the HIV non-LD group. Whilst the majority of patients in the HIV LD and non-LD groups were MSM

( $83 \%$ and $76 \%$ respectively) some other risk factors were seen including intravenous drug abuse, heterosexual contact and blood contamination.

\begin{tabular}{|c|c|c|c|c|c|c|c|c|}
\hline & \multicolumn{3}{|c|}{ HIV LD } & \multicolumn{3}{|c|}{ HIV non-LD } & \multirow{2}{*}{ MSM } & \multirow{2}{*}{ p-value } \\
\hline & Male & Female & Total & Male & Female & Total & & \\
\hline \multicolumn{9}{|l|}{ N } \\
\hline Recruited & 41 & 15 & 56 & 49 & 13 & 62 & 54 & $<0.001$ \\
\hline Age (years) & 45 & 41 & 44 & 41 & 33 & 39 & 35 & $<0.001$ \\
\hline \multicolumn{9}{|l|}{ Unit N (\%) } \\
\hline Edinburgh & $32(78)$ & $13(87)$ & $45(80)$ & $11(22)$ & $1(8)$ & $12(19)$ & $38(70)$ & \multirow{2}{*}{$<0.001$} \\
\hline Glasgow & $9(22)$ & $2(13)$ & $11(20)$ & $38(78)$ & $12(92)$ & $50(81)$ & $16(30)$ & \\
\hline \multicolumn{9}{|l|}{ Ethnicity N (\%) } \\
\hline Caucasian & $41(100)$ & $14(93)$ & $55(98)$ & $43(88)$ & $8(62)$ & $51(82)$ & $53(98)$ & \multirow{4}{*}{0.013} \\
\hline African & 0 & $1(7)$ & $1(2)$ & $3(6)$ & $5(38)$ & $8(13)$ & 0 & \\
\hline Thai & 0 & 0 & 0 & $2(4)$ & 0 & $2(3)$ & $1(2)$ & \\
\hline Malaysian & 0 & 0 & 0 & $1(2)$ & 0 & $1(2)$ & 0 & \\
\hline \multicolumn{9}{|l|}{ Risk Factors N (\%) } \\
\hline MSM & $34(83)$ & 0 & $34(61)$ & $37(76)$ & 0 & $37(60)$ & & \multirow{5}{*}{0.472} \\
\hline IVDA & $2(5)$ & $5(33)$ & $7(13)$ & $2(4)$ & $1(8)$ & $3(5)$ & & \\
\hline Heterosexual contact & $4(10)$ & $10(77)$ & $14(25)$ & $9(18)$ & $11(85)$ & $20(32)$ & & \\
\hline Mother to child & 0 & 0 & 0 & 0 & $1(8)$ & $1(2)$ & & \\
\hline Blood contamination & $1(2)$ & 0 & $1(2)$ & $1(2)$ & 0 & $2(3)$ & & \\
\hline Duration of HIV (years) & 10.7 & 15.7 & 11.9 & 6.5 & 4.1 & 6.2 & & $<0.001$ \\
\hline $\begin{array}{l}\text { Duration of HAART } \\
\text { (years) }\end{array}$ & 8.3 & 8.2 & 8.2 & 2.3 & 1.7 & 2.2 & & $<0.001$ \\
\hline \multicolumn{9}{|l|}{$\begin{array}{l}\text { Viral load (HIV copies/ml) } \\
\text { N }(\%)\end{array}$} \\
\hline Undetectable & $39(95)$ & $12(80)$ & $51(91)$ & $44(89)$ & $12(92)$ & $56(90)$ & & \multirow{3}{*}{0.768} \\
\hline Detectable & $2(5)$ & $3(20)$ & $5(9)$ & $5(11)$ & $1(8)$ & $7(10)$ & & \\
\hline Mean detectable & 1100 & 18933 & 10016 & 104263 & 24700 & 64481 & & \\
\hline $\begin{array}{l}\text { CD4 count, cell } / \mathrm{mm}^{3} \\
\text { (mean +- SD) }\end{array}$ & 482 & 596 & 514 & 465 & 459 & 464 & & 0.26 \\
\hline BMI & 22.1 & 20.5 & 21.3 & 24.1 & 26.6 & 25.3 & & $<0.001$ \\
\hline
\end{tabular}

Thus, analysis of psychological scores was performed on the groups as a whole, and following exclusion of non-MSM and non-Caucasian individuals. Patients in the HIV LD group had a longer duration of HIV infection (5-year difference) and treatment with 
Highly Active Antiretroviral Therapy (6-year difference) than HIV non-LD patients although no difference in viral load and CD4 count were seen. The mean BMI of HIV LD group was also slightly lower than the HIV non-LD group.

Due to the small number of females recruited to each group, females were excluded from statistical comparison of psychological measures between groups. This was decided on the basis that no meaningful conclusions could be drawn from comparison of such small samples. Comparison of the psychological scores (DAS-24, HADS-A and HADS-D) for the groups as a whole and following exclusion of non-MSM and nonCaucasian individuals demonstrated no difference in results. Therefore, results for analysis of subgroups excluding non-MSM and non-Caucasian individuals are presented.

\subsubsection{Derriford Appearance Scale}

Baseline DAS-24 scores for the HIV LD cohort and control groups are summarized in Table 4.2. Dotplots of the DAS and HADS results are provided in appendix $\mathrm{x}$ and demonstrate that, despite a large range and standard deviation for some groups, there is reasonable spread of the data with no particular outliers. Analysis using ANOVA tests demonstrated significantly increased scores for the HIV LD group compared to male HIV non-LD patients and the MSM group. There was no significant difference in scoring between male HIV non-LD and MSM subgroups ( $\mathrm{p}=0.83$ ).

Table 4.2. DAS-24 scores by subgroup with p-values from ANOVA tests

\begin{tabular}{lcc}
\hline HIV & $\mathrm{N}$ & 41 \\
LD & Mean (SD) & $55.5(16.5)$ \\
\hline HIV & $\mathrm{N}$ & 49 \\
non- & Mean (SD) & $35.3(12.7)$ \\
LD & $\mathrm{N}$ & 54 \\
MSM & Mean (SD) & $35.8(11.0)$ \\
\hline & p-value & $\mathrm{p}<0.001$ \\
\hline
\end{tabular}


The DAS-24 reference data for males aged 31-60 years in the general population and in a clinical population of head and neck patients were extracted for comparison with the study population groups (Figure 4.2).

Figure 4.2. Summary of DAS-24 male participant scores versus population reference values

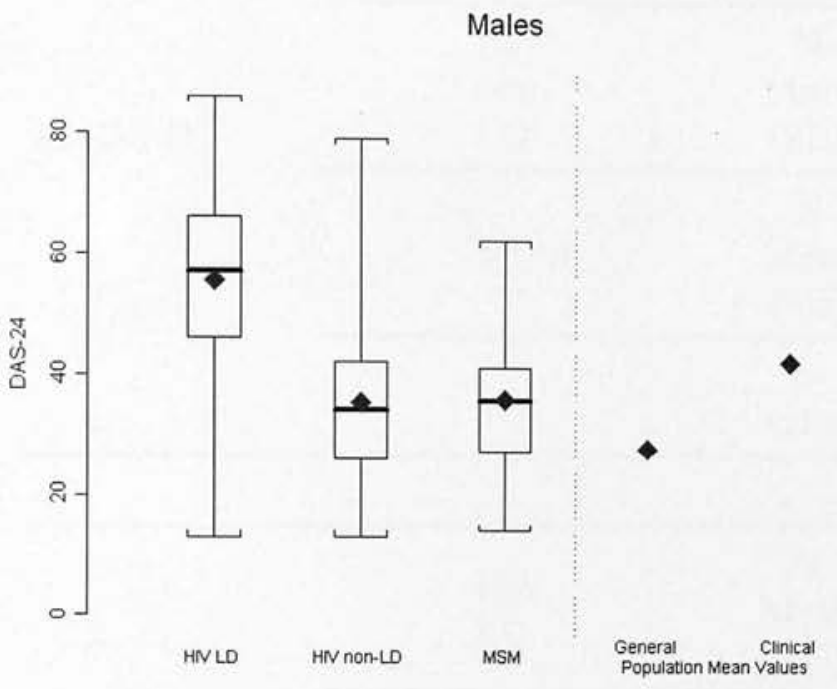

Mean scores for HIV LD patients (55) were higher than males in the general population (27) and clinical population of head and neck patients (41) $(\mathrm{p}<0.001)$. Mean scores for the HIV non-LD group (35) and MSM group (35) were also significantly higher than the general population $(\mathrm{p}<0.001)$ but lower than the clinical head and neck population $(\mathrm{p}=0.03)$.

\subsubsection{Hospital Anxiety and Depression Scale}

HADS-D and HADS-A scores are summarized in Table 4.3 and Figure 4.3.

The male HIV LD group had higher depression scores than the male HIV non-LD and MSM groups $(\mathrm{p}<0.001)$. There was no difference in scoring between male HIV non-LD and MSM subgroups $(\mathrm{p}=0.92)$. A similar pattern was seen for anxiety scores, though the statistical evidence for differences between the male subgroups was not significant $(\mathrm{p}=0.070)$. 
Table 4.3. HADS-D and HADS-A scores by subgroup with p-values from ANOVA tests

\begin{tabular}{|c|c|c|c|}
\hline \multirow{4}{*}{ HADS-D } & $\begin{array}{l}\text { HIV } \\
\text { LD }\end{array}$ & $\begin{array}{c}\mathrm{N} \\
\text { Mean } \\
\text { (SD) }\end{array}$ & $\begin{array}{c}41 \\
7.6 \\
(5.8)\end{array}$ \\
\hline & $\begin{array}{l}\text { HIV } \\
\text { non- } \\
\text { LD }\end{array}$ & $\begin{array}{c}\mathrm{N} \\
\text { Mean } \\
\text { (SD) }\end{array}$ & $\begin{array}{c}49 \\
3.5 \\
(4.3)\end{array}$ \\
\hline & MSM & $\begin{array}{c}\mathrm{N} \\
\text { Mean } \\
\text { (SD) }\end{array}$ & $\begin{array}{c}53 \\
3.5 \\
(3.0)\end{array}$ \\
\hline & & $\begin{array}{c}\mathrm{p}- \\
\text { value }\end{array}$ & $\mathrm{p}<0.001$ \\
\hline \multirow{4}{*}{ HADS-A } & $\begin{array}{l}\text { HIV } \\
\text { LD }\end{array}$ & $\begin{array}{c}\mathrm{N} \\
\text { Mean } \\
\text { (SD) }\end{array}$ & $\begin{array}{c}41 \\
9.8 \\
(5.3)\end{array}$ \\
\hline & $\begin{array}{l}\text { HIV } \\
\text { non- } \\
\text { LD }\end{array}$ & $\begin{array}{c}\mathrm{N} \\
\text { Mean } \\
\text { (SD) }\end{array}$ & $\begin{array}{c}49 \\
7.6 \\
(5.3)\end{array}$ \\
\hline & MSM & $\begin{array}{c}\mathrm{N} \\
\text { Mean } \\
\text { (SD) }\end{array}$ & $\begin{array}{c}53 \\
7.8 \\
(4.0) \\
\end{array}$ \\
\hline & & $\begin{array}{c}\mathrm{p}- \\
\text { value }\end{array}$ & $\mathrm{p}=0.070$ \\
\hline
\end{tabular}




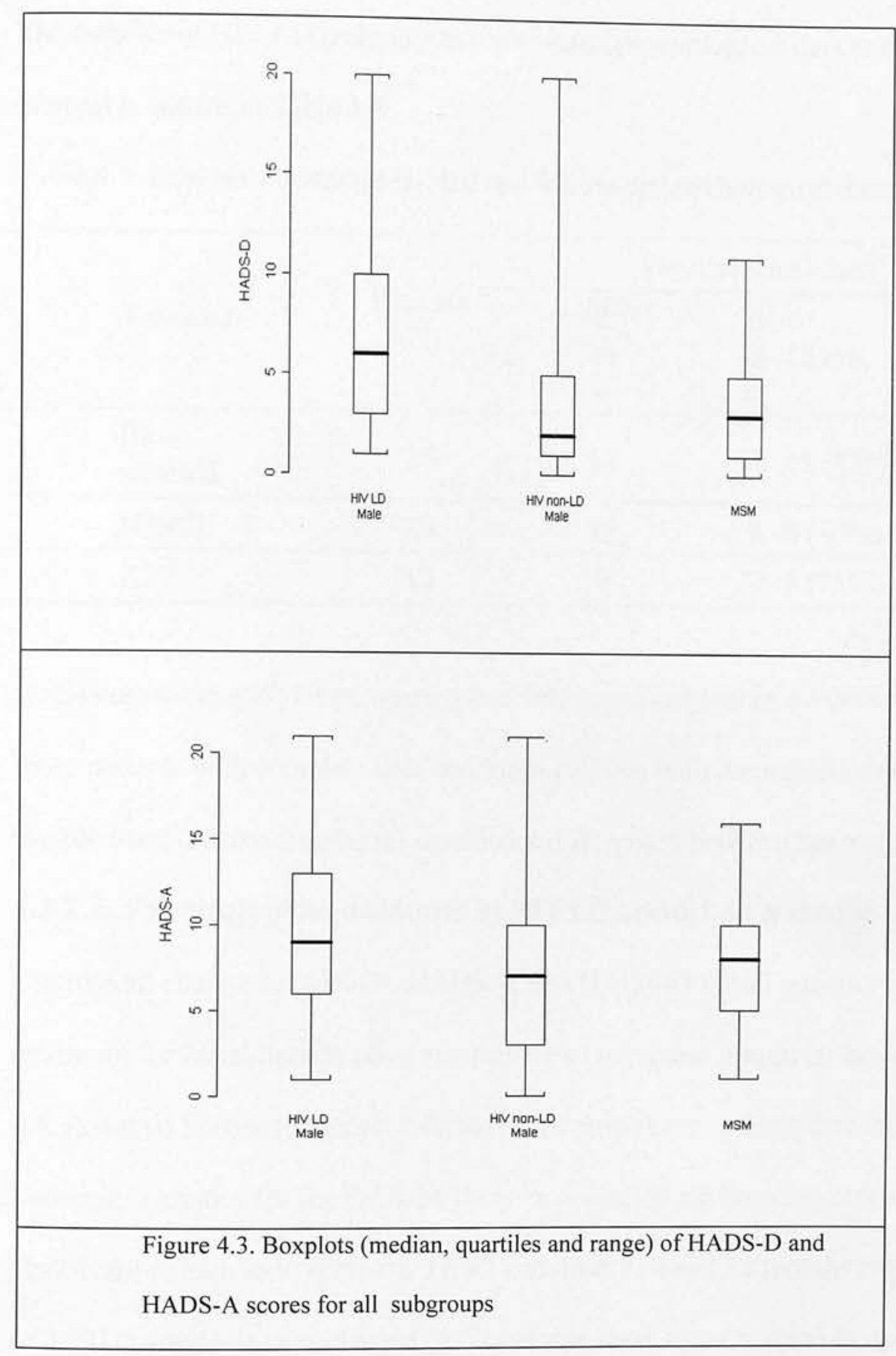

\subsubsection{Psychological outcome in HIV LD treatment cohort}

Forty-eight patients from the HIV LD cohort underwent treatment for HIV facial lipoatrophy: 16 patients had Bio-alcamid augmentation, 20 patients received Newfill injections and 12 patients underwent fat transfer. The demographic and clinical data for patients in each treatment group is summarized in table and discussed in Chapter 2.

\subsubsection{Data completion}


The number of HIV LD patients who completed psychological data at each follow-up interval is shown in Table 4.4 .

Table 4.4. Number of patients treated and follow-up psychological data completed

\begin{tabular}{lcccc}
\hline & & \multicolumn{3}{c}{ Psychological data } \\
\cline { 3 - 5 } Treatment & Patients & 2 & 6 \\
& $(\mathrm{~N})$ & $\begin{array}{c}2 \\
\mathrm{~m}\end{array}$ & $\begin{array}{c}6 \\
\mathrm{~m}\end{array}$ & $12 \mathrm{mo}$ \\
\hline Bio- & & 15 & 12 & $13(81 \%)$ \\
\hline alcamid & 16 & 16 & 8 & $8(40 \%)$ \\
\hline Newfill & 20 & 9 & 12 & $9(75 \%)$ \\
\hline AFT & 12 & &
\end{tabular}

A missing-value analysis comparing baseline psychological measurements between those patients with complete data and those patients with incomplete data (using twosample t-tests) demonstrated no significant differences between the two groups.

\subsubsection{Psychological outcome in HIV LD cohort as a whole}

The overall change in DAS-24, HADS-A and HADS-D for all patients undergoing treatment for facial lipoatrophy (irrespective of treatment group) is shown in Figure 4.4. A dotted line on the graph indicates 'caseness'scores greater than 11 for HADS. Summary statistics for the DAS-24 show an overall mean baseline score of 54 with a significant reduction in scores to 39, 42 and 43 at 2, 6 and 12 months respectively (all $\mathrm{p}<0.001$; paired t-tests vs. baseline). There was some improvement in overall HADS-A scores compared to baseline although results did not reach significance at 12 months $(\mathrm{m} 2 \mathrm{p}=0.058, \mathrm{~m} 6: \mathrm{p}=0.082, \mathrm{~m} 12: \mathrm{p}=0.184)$. There was a significant improvement in overall HADS-D scores compared to baseline ( $\mathrm{p}=0.004,0.003,0.027$ at months 2,6 and 12 respectively, vs. baseline). 
Figure 4.4. Summary of psychological measures of all HIV LD patients undergoing treatment for facial lipoatrophy (dotted line indicates 'caseness' scores for HADS greater than 11).

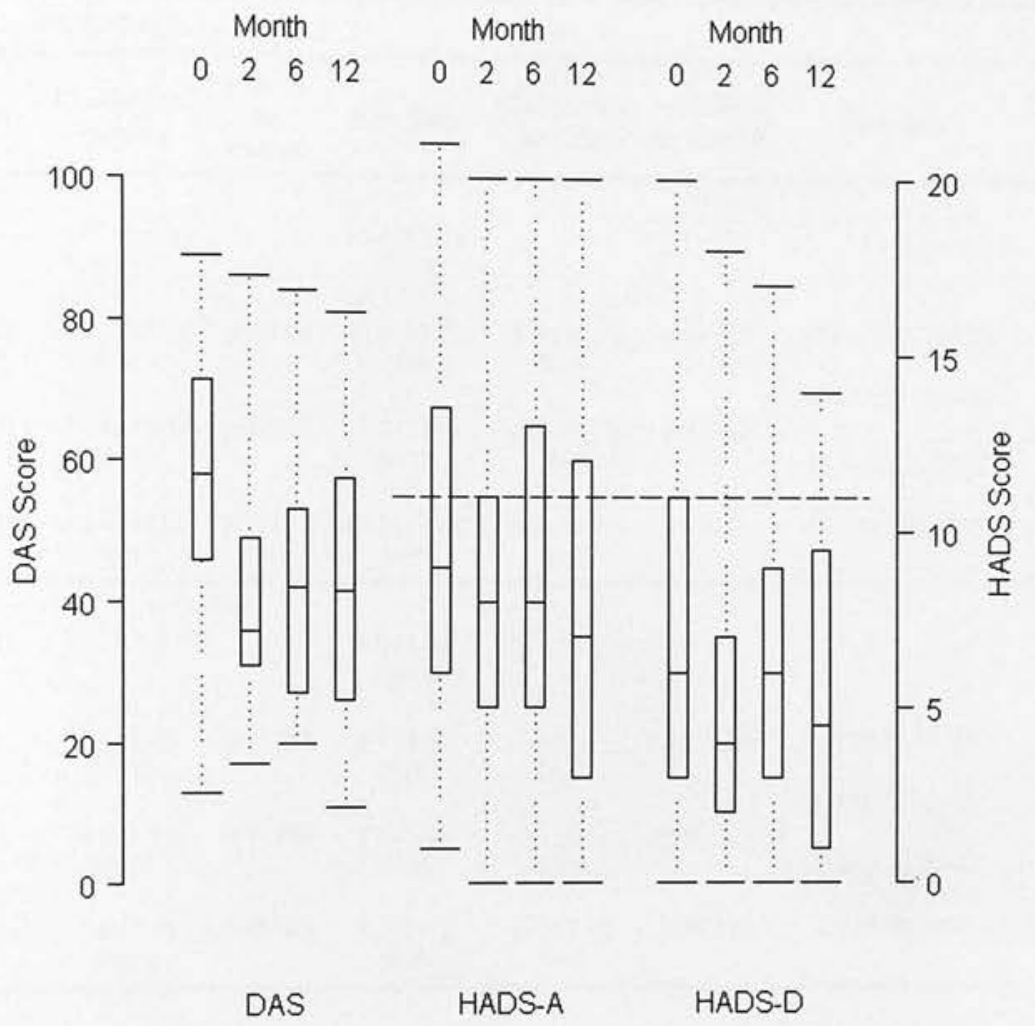

\subsubsection{Psychological outcome in each HIV LD treatment group}

Derriford Appearance Scale

Table 4.5 provides summary statistics for DAS scores by treatment and follow-up interval. In addition, the change in scores relative to baseline with t-test $\mathrm{p}$-values for test of zero change is shown. 
Table 4.5 Mean, SD and number of observations of DAS, HADS-A and HADS-D, by visit and treatment type. Mean, SD and number of observations of changes over baseline, with paired t-test p-values for test of zero change.

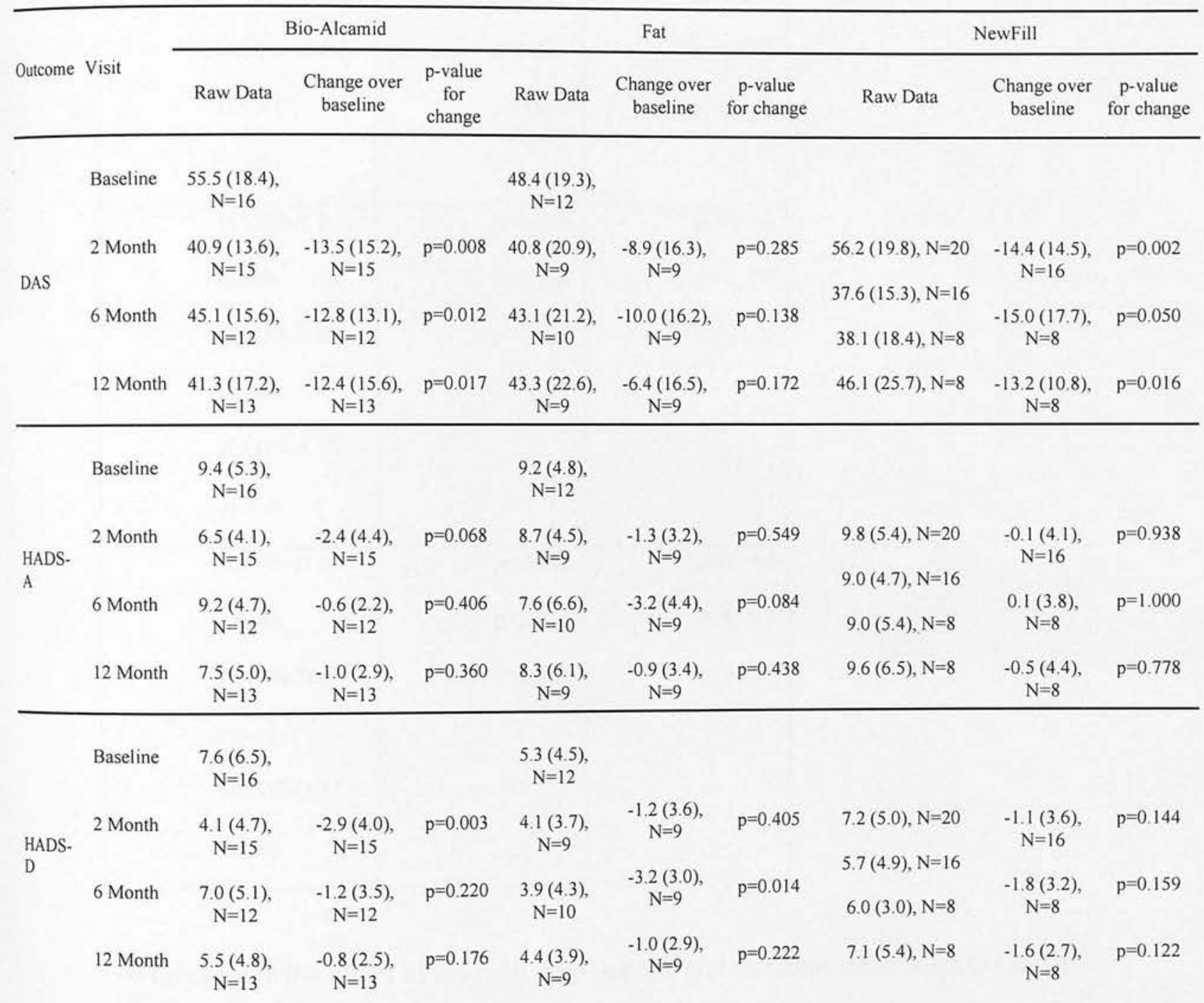

Comparison of psychological measures between treatment groups using ANOVA

demonstrated no significant difference in DAS-24 scores at baseline or throughout

follow-up (Table 4.6). 
Table 4. 6. p-values for tests of no difference between groups in mean value or mean change over baseline using ANOVA

\begin{tabular}{|l|l|l|}
\hline Follow-up & Mean & Change \\
\hline DAS 2 month & $\mathrm{p}=0.701$ & $\mathrm{p}=0.610$ \\
DAS 6 month & $\mathrm{p}=0.821$ & $\mathrm{p}=0.256$ \\
DAS 12 & $\mathrm{p}=0.891$ & $\mathrm{p}=0.145$ \\
month & $\mathrm{p}=0.225$ & \\
\hline HADS-A 2 & $\mathrm{p}=0.336$ & $\mathrm{p}=0.330$ \\
month & $\mathrm{p}=0.576$ & $\mathrm{p}=0.258$ \\
HADS-A 6 & & $\mathrm{p}=0.110$ \\
month & $\mathrm{p}=0.210$ & $\mathrm{p}=0.508$ \\
HADS-A 12 & $\mathrm{p}=0.915$ & $\mathrm{p}=0.220$ \\
month & & \\
\hline HADS-D 2 & & \\
month & & \\
HADS-D 6 & & \\
month & & \\
HADS-D 12 & & \\
\hline month & & \\
\hline
\end{tabular}

To investigate the effect of the high drop out rate and resultant small sample size at follow-up, linear mixed effects regression models were applied to each psychological measure, in the same manner as for facial volumes. Model-predicted values at each time point for each treatment group, with $95 \%$ confidence intervals demonstrated little difference compared to the raw data and are therefore provided in Appendix 3 for reference only.

For Bio-alcamid, a significant difference in DAS scores compared to baseline was found at all time intervals. In the fat group, improvements in DAS scores were also 
demonstrated at all follow-up visits, although the values did not reach significance as a consequence of small sample size. Significant improvements in DAS scores were found at all time intervals in the Newfill group, although there was in increase in scores at 12 months.

Figure 4.5. Box plot of DAS-24 results for each treatment group over time

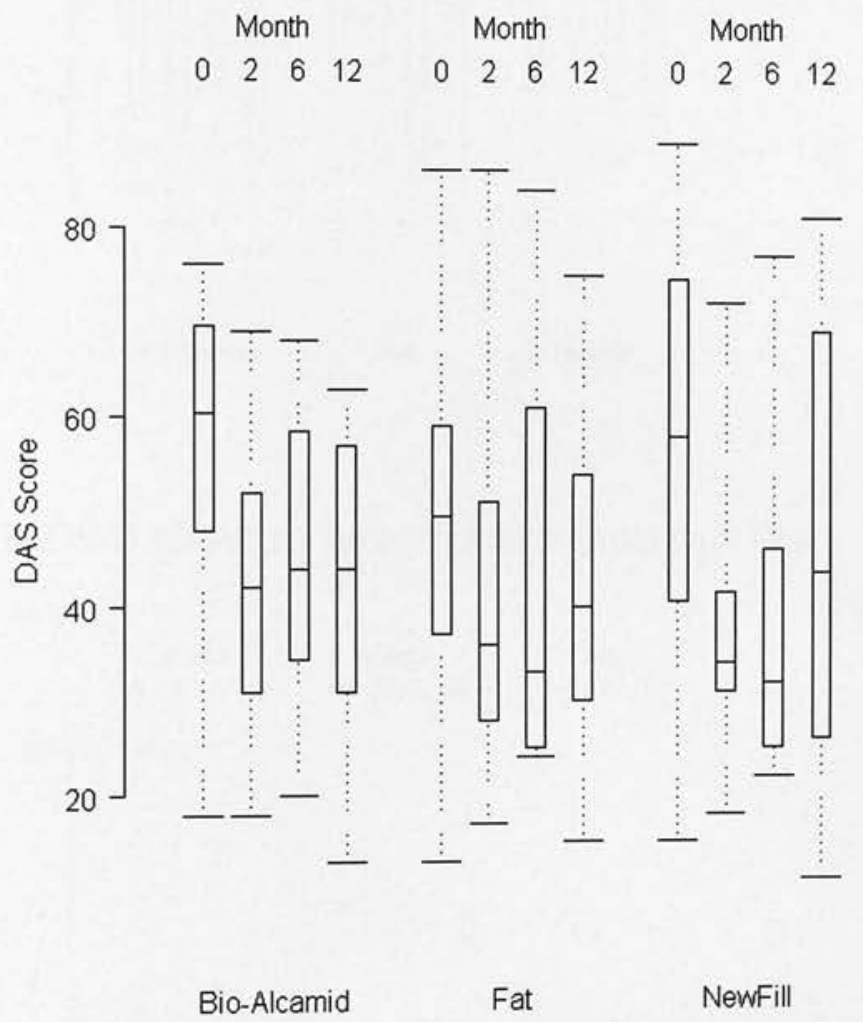

\section{Hospital Anxiety and Depression Scale}

Mean HADS-A and HADS-D scores are summarized by treatment group and follow-up interval (including patient numbers) in Table 4.5. No significant difference in HADS-A or HADS-D scores between the 3 treatment groups at baseline or throughout follow-up (Table 4.6). Figures 4.6 and 4.7 show boxplots of HADS-A and HADS-D for each treatment group over time. 
Figure 4.6. HADS-A results for each treatment group over time
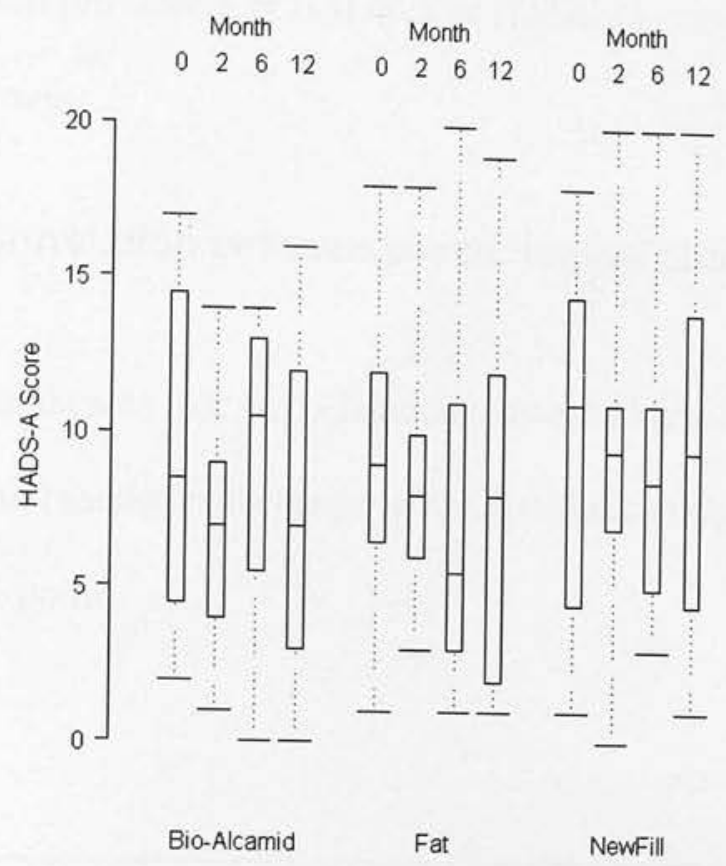

Figure 4.7. HADS-D results for each treatment group over time

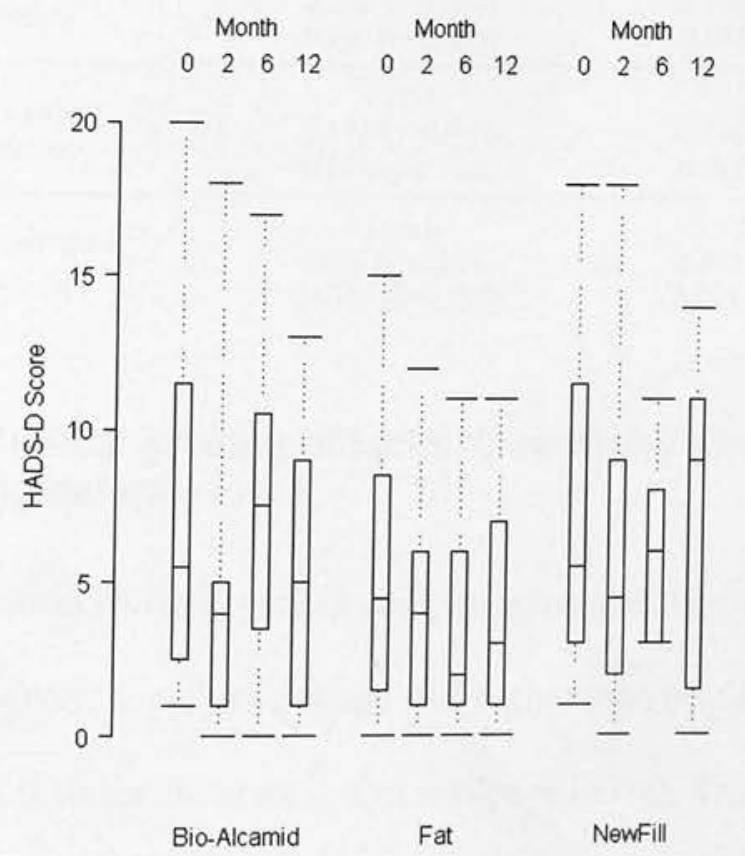

In the Bio-alcamid group, post-treatment scores reached significance for HADS-D at 2 months only. Some improvement in HADS-A and HADS-D scores was found in the fat 
group, although values only reached significance for HADS-D at 6 months. No significant improvement in HADS-A or HADS-D scores was demonstrated for the Newfill group.

\subsubsection{Correlation between psychological outcome and facial volume change}

Table 4.7 illustrates that no correlation between change in psychological measures compared to baseline and change in facial volume measured by 3-D imaging was seen at any time point.

Table 4.7: Correlations between changes in volume and changes in psychological factors.

\begin{tabular}{cccc}
\hline $\begin{array}{l}{[\mathrm{N}]} \\
\text { Pearson } \text { Corr }^{\mathrm{n}} \text { (p-value) } \\
\text { Rank Corr }^{\mathrm{n}} \text { (p-value) }\end{array}$ & DAS Change & HADS-A Change & HADS-D Change \\
\hline 2 months - & {$[32]$} & {$[32]$} & {$[32]$} \\
Baseline & $0.212(\mathrm{p}=0.244)$ & $-0.121(\mathrm{p}=0.509)$ & $-0.053(\mathrm{p}=0.775)$ \\
& $0.146(\mathrm{p}=0.416)$ & $-0.161(\mathrm{p}=0.369)$ & $-0.062(\mathrm{p}=0.728)$ \\
\hline 6 months & {$[20]$} & {$[20]$} & {$[20]$} \\
Baseline & $0.139(\mathrm{p}=0.560)$ & $-0.010(\mathrm{p}=0.968)$ & $0.118(\mathrm{p}=0.621)$ \\
& $0.050(\mathrm{p}=0.829)$ & $-0.068(\mathrm{p}=0.764)$ & $0.107(\mathrm{p}=0.644)$ \\
\hline \multirow{2}{*}{ 12 months } & {$[16]$} & {$[16]$} & {$[16]$} \\
Baseline & $0.036(\mathrm{p}=0.894)$ & $-0.067(\mathrm{p}=0.805)$ & $0.168(\mathrm{p}=0.535)$ \\
& $-0.046(\mathrm{p}=0.855)$ & $0.083(\mathrm{p}=0.752)$ & $0.082(\mathrm{p}=0.756)$
\end{tabular}

\subsubsection{Clinical grading of facial lipoatrophy and correlation with psychological measures}

The correlation between patient and physician grading of facial lipoatrophy is shown in

Table 4.8. Overall, patient grading was higher than physician grading and the correlation between these measures was poor $(63 \%)$. The correlation between psychological outcome and both patient and physician grading of severity is summarised in Tables 4.9 - 4.10. A pattern of greater psychological scores for DAS, HADS-A and HADS-D was found with higher grading of facial lipoatrophy, (particularly patient grading) although this only reached significance for HADS-D. 
Table 4.8. Patient vs. Physician Grading of severity.

\begin{tabular}{|c|c|c|c|c|c|}
\hline & & & \multicolumn{3}{|c|}{ Physician Grading } \\
\hline & & & 1 & 2 & 3 \\
\hline \multirow{3}{*}{\multicolumn{2}{|c|}{ Patient Grading }} & 1 & 6 & 0 & 0 \\
\hline & & 2 & 3 & 12 & 0 \\
\hline & & 3 & 2 & 11 & 9 \\
\hline \multicolumn{3}{|c|}{ Percentage Agreement } & & $62.8 \%$ & \\
\hline \multicolumn{3}{|c|}{ McNemar's Test } & & $\mathrm{p}<0.001$ & \\
\hline & & \multicolumn{3}{|c|}{ Patient Grading } & \multirow{2}{*}{$\begin{array}{l}\text { ANOVA } \\
\text { p-value }\end{array}$} \\
\hline & & 1 & 2 & 3 & \\
\hline \multirow[b]{2}{*}{ DAS } & {$[\mathrm{N}]$} & [6] & {$[15]$} & [22] & \multirow[b]{2}{*}{$\mathrm{p}=0.107$} \\
\hline & $\begin{array}{l}\text { Mean } \\
\text { (SD) }\end{array}$ & $\begin{array}{c}44.67 \\
(13.08)\end{array}$ & $\begin{array}{c}49.53 \\
(17.62)\end{array}$ & $\begin{array}{c}59.32 \\
(18.24)\end{array}$ & \\
\hline \multirow{2}{*}{$\begin{array}{l}\text { HADS- } \\
\text { A }\end{array}$} & {$[\mathrm{N}]$} & [6] & [15] & [22] & \multirow[b]{2}{*}{$\mathrm{p}=0.669$} \\
\hline & $\begin{array}{l}\text { Mean } \\
\text { (SD) }\end{array}$ & $\begin{array}{c}8.50 \\
(5.17)\end{array}$ & $\begin{array}{c}9.00 \\
(536)\end{array}$ & $\begin{array}{l}10.23 \\
(4.94)\end{array}$ & \\
\hline \multirow{3}{*}{$\begin{array}{l}\text { HADS- } \\
\text { D }\end{array}$} & & & & & \multirow{3}{*}{$\mathrm{p}=0.043$} \\
\hline & Mean & 4.33 & 5.20 & 8.91 & \\
\hline & (SD) & $(2.42)$ & $(4.13)$ & $(5.93)$ & \\
\hline & & \multirow{2}{*}{\multicolumn{3}{|c|}{ Physician Grading }} & \multirow{3}{*}{$\begin{array}{c}\text { ANOVA } \\
\text { p-value }\end{array}$} \\
\hline & & & & & \\
\hline & & 1 & 2 & 3 & \\
\hline \multirow[b]{2}{*}{ DAS } & {$[\mathrm{N}]$} & [11] & [23] & [9] & \multirow[b]{2}{*}{$\mathrm{p}=0.703$} \\
\hline & $\begin{array}{l}\text { Mean } \\
\text { (SD) }\end{array}$ & $\begin{array}{c}51.55 \\
(16.32)\end{array}$ & $\begin{array}{c}53.26 \\
(18.37)\end{array}$ & $\begin{array}{c}58.22 \\
(20.33)\end{array}$ & \\
\hline \multirow{3}{*}{$\begin{array}{l}\text { HADS- } \\
\text { A }\end{array}$} & {$[\mathrm{N}]$} & [11] & [23] & [9] & \multirow{3}{*}{$p=0.628$} \\
\hline & Mean & 8.36 & 10.17 & 9.44 & \\
\hline & (SD) & $(4.86)$ & $(5.37)$ & $(4.67)$ & \\
\hline \multirow{2}{*}{$\begin{array}{l}\text { HADS- } \\
\text { D }\end{array}$} & {$[\mathrm{N}]$} & [11] & [23] & [9] & \multirow[b]{2}{*}{$p=0.416$} \\
\hline & $\begin{array}{l}\text { Mean } \\
\text { (SD) }\end{array}$ & $\begin{array}{c}5.18 \\
(3.06)\end{array}$ & $\begin{array}{c}7.78 \\
(6.30)\end{array}$ & $\begin{array}{c}7.11 \\
(4.46)\end{array}$ & \\
\hline
\end{tabular}




\subsection{Discussion}

\subsubsection{Selection of control groups}

The psychological distress caused by HIV-associated lipodystrophy is a wellrecognised problem. This study attempted to quantify the effect of various factors influencing body image assessment and psychological outcome in this group. A group of HIV patients without lipodystrophy, but matched to the cohort group with respect to other variables was required to evaluate the impact of lipodystrophy on psychological distress. In addition, a control group of non-HIV patients was a requirement to investigate the effect of HIV infection as an independent variable. Given that a significant proportion of referrals made to the Plastic Surgery Unit in Edinburgh since the introduction of treatment funding for facial lipoatrophy were MSM, sexual orientation was also hypothesized to potentially influence body image perception. Therefore, in an attempt to keep the non-HIV group matched to the cohort group with respect to this variable, the inclusion of an MSM group was devised. The authors of the DAS scale provide reference tables based on large population studies, which account for age, sex and ethnic status. However, the impact of sexual orientation, hypothesized to influence body image perception and referral for treatment has not previously been assessed using this scale. This rationale formed the basis for selection of the control groups as discussed in the methods section.

Consideration should be given however to the baseline demographics of patients recruited to each study group. When analyzing the results, the risk factors were more variable than anticipated. Although the greatest proportion in each group was MSM ( $83 \%$ of HIV LD and $76 \%$ of HIV non-LD), there were other risk factors displayed in the patient demographics such as IVDA ( $5 \%$ in the HIV LD and $4 \%$ in the HIV nonLD) and heterosexual contact (10\% in the HIV LD and $18 \%$ in the HIV non-LD). 
Therefore, the results of this study are skewed, given that one of the control groups is comprised of only MSM patients and cannot be compared to the other control groups who demonstrate variable risk factors. Considering IVDA patients for example, different levels of psychological distress or body image perceptions may exist compared to MSM patients due to the complex interplay of social and lifestyle factors. Analysis of psychological measures in this study with exclusion of non-MSM and nonCaucasians demonstrated no difference compared to the groups as a whole. However, the sample size may be too small to detect a difference between groups. Inclusion of a control group matched for these other risk factors would have been helpful. However, a much larger sample size would be required to test the difference between the risk factor variables statistically.

\subsubsection{Psychological characteristics of cohort versus control}

\section{groups at baseline and confounding variables}

In summary, comparison of all three male subgroups, demonstrated significantly higher scores for the DAS-24 and HADS-D in patients with HIV lipodystrophy patients compared to controls. Scores for HADS-A were also higher, although not significant. It is interesting to note that no difference in DAS-24, HADS-D or HADS-A scores was evident between the HIV non-LD males and MSM group. However, when interpreting these findings, consideration of other confounding variables influencing body image perception such as age, gender and ethnic origin of subjects is particularly relevant. The mean age of the patients in the HIV LD group was similar to the HIV non-LD group but 10 years older than the MSM group. The published norm tables for the DAS-24 show greater scores for the general male population in a younger age group (median = 26 in 18-30 yrs) compared to the older age groups (median $=25$ in 31-60 years, median $=20$ in 61 years and over). If using the norm tables for comparison then the mean age 
of all 3 male subgroups falls into the 31-60 year age range. Therefore, one drawback of the reference tables is the relatively wide range of this age subdivision. It would be useful to know if a 10-year age difference (such as that displayed between the MSM group and the other control groups) has any effect on DAS scores. Therefore, the DAS24 scores for the younger MSM group may not be comparable to the other subgroups. It is possible that selection of an MSM group matched for age would display lower DAS-24 scores.

All of the male patients in the HIV LD group were Caucasian and similar numbers of Caucasian patients were recruited to the control groups. A small number of patients of different ethnic origins were recruited to the HIV non-LD and MSM group but exclusion of these patients did not affect results. However, it is possible that a larger sample size would demonstrate differences between ethnic groups for the selected scales. For example, the features that determine attractiveness and importance placed on body image may vary be subject to cultural variation. The morphological changes attributable to HIV lipodystrophy may therefore be therefore be associated with different levels of psychological distress depending on cultural perceptions of attractiveness. In addition, there Rumsey reports evidence for increased appearance related concern in 'non-White' ethnic origin living in the UK is based on difficulties with social encounters and employment issues (Rumsey, Clarke et al. 2004).

The effect of gender on psychological outcome has not been investigated in this study but is another important variable to consider. Further investigation of psychological measures in a larger female population of HIV-seropositive patients with and without lipodystrophy would be useful as the number of participants in this study were limited. Indeed, one study has found that women may be more sensitive than men in terms of psychological difficulty following disfigurement (Anderson and Maksud 1994). 
When comparing baseline demographics between groups, the proportion of patients recruited from Edinburgh and Glasgow differed significantly. Thus, geographical variation between groups may be an additional source of bias. It is possible that socioeconomic factors differ in patients recruited from these different sites. The general health literature suggests that those at a lower socio-economic level are more affected by their health status (Marmot, Smith et al. 1991). It has also been found that the unemployed are at a greater risk of developing psychological distress following acute injury (Joy, Probert et al. 2000).

Although the limitations of the control groups have been discussed, the results of the study do highlight the significantly greater levels of the psychological distress in the HIV LD group. The DAS-24 scores for the HIV LD group were also markedly higher than for a clinical population with other facial disfigurements, which reinforces the significant stigmatizing nature of this condition. Utilizing the reference tables for DAS24 , mean scores for all male subgroups in this study were higher than the general male population. This suggests that there may be greater concern over appearance in the MSM group compared to males in the general population. However, the reference values do not represent the local population and a further, larger study with matching of other confounding variables would be required to draw conclusions on the significance of sexual orientation on body image perception as an independent variable.

\subsubsection{Psychological measures}

\subsubsection{Selection of psychological measures}

The HADS scale was selected for this study for several reasons:

- established reliability and validity

- the scale is limited to 14 items which makes it easy to administer and well accepted 
- the scale has no items relating to somatic symptoms that may confound the diagnosis in patients who also have physical illness and therefore reduce the sensitivity in screening for depression

- ability of the test to detect change over time

- availability of reference values for a variety of medical and non-medical populations. The HADS has gained widespread use in clinical practice and has demonstrated excellent acceptability, reliability and validity, particularly in 2 large meta-analysis reviews of the scale based on 747 and 200 papers in each review respectively (Herrmann 1997; Bjelland, Dahl et al. 2003). There has been some discussion about whether the anxiety and depression subscales really measure different aspects of mood. Some investigators have preferred the HADS total score as a general measure of distress (Chaturvedi 1991), others have found no significant correlation between the two subscales. (Aylard, Gooding et al. 1987). In fact, the mean correlation between anxiety and depression subscales from 18 separate studies with $\mathrm{n}=8160$ is $\mathrm{r}=0.63$. Thus, subscale scores are clearly correlated in most patient groups. Given the extensive comorbidity between anxiety and depression, the moderate to strong correlations between HADS-A and HADS-D subscales reported are to be expected. Thus the total HADS scale could have been used as an alternative method of analyzing results in this study. A further analysis could also have incorporated the correlation between HADS-A, HADS-D and DAS scores. However, the results of the literature review support the two-factor structure of HADS and there is sufficient evidence that both subscales differ in a clinically meaningful way. Although there is some overlap, it is recommended that both subscales should therefore be used separately for most purposes. In addition, presentation of the data in separate subscales facilitates comparison of results with reference data in different clinical studies. Interestingly, no differences in HADS-A scores were demonstrated either between the HIV LD cohort and controls or when 
comparing the three different treatment groups. This may represent a real finding but could also result from inadequacy of the scale to detect anxiety symptoms in these groups. However, the excellent sensitivity and specificity of the HADS as a case finder for anxiety and depression was also reported in the review papers (Herrmann 1997; Bjelland, Dahl et al. 2003). The optimal threshold values for 'case-finders' has been shown to be $8+$ as suggested in the original paper on HADS. In this study, values above this threshold were seen for HADS-A in the HIV LD group but not for HADS-D at baseline. Post-treatment HADS-A values fell below the threshold at 2 and 12 months. Although results were not found to be statistically significant, a reduction in scores below the 'caseness' cut-off may represent a positive clinical intervention.

Finally, the benefits and drawbacks of HADS in this study over other psychological measures should be highlighted. Several studies have demonstrated high correlation of the HADS subscales with scales used as gold standards for depression and anxiety such as the Beck Depression Inventory (BDI) and the State Trait Anxiety Inventory (STAI). (Michopolous, Douzenis et al. 2008)

Meta-analysis comparing HADS with additional scales revealed that HADS exhibited similar sensitivity and specificity as longer versions of the General Health Questionaire. When compared to other questionnaires for anxiety and depression in common use such as BDI, STAI, CAS, and SCL-90 Anxiety and Depression subscales, the

correlation to HADS-D and HADS-A, respectively, were between .60 and .80 , which should be characterised as medium to strong correlations (Bjelland, Dahl et al. 2003). Thus, the concurrent validity of HADS is considered to be very good. An additional benefit to the use of HADS in this study is the exclusion of somatic items. Because there is a large overlap between HIV manifestations and somatic symptoms of anxiety and depressions, it is crucial to use measures that do not contain somatic items to 
validly and reliably assess these psychological states in HIV-infected patients. One study evaluated the psychometric properties of the HADS in 162 HIV-seropositive patients, who, in addition to the HADS, also completed the Beck Depression Inventory (BDI) and the State-Trait Anxiety Inventory (Savard, Laberge et al. 1998). In contrast to the BDI, HADS scores were found to be unconfounded by the presence of HIV symptomatology. The authors conclude that the HADS appears to represent the best currently available self-report scale, to reliably and validly assess anxiety and depression in HIV-infected patients.

Therefore, the choice of HADS as a measure of anxiety and depression in this study was good overall. The addition of a total HADS score may have been beneficial. It is possible that the total score may have demonstrated a more convincing improvement following treatment intervention than either sub-scale individually.

The advantages of utilizing the DAS-24 scale in this study were:

- the short form is quick to complete thus more acceptable to patients and scores can easily be collated

- the scale is condition-specific, developed specifically for assessing concern about physical appearance

- the scale has been validated and tested in a large population of plastic surgery patients

- reference data is available for general and clinical populations based on age and sex However, several drawbacks of the scale emerged during the study. The greatest disadvantage of the DAS-24 is that the scale is not specific to facial lipoatrophy. One systematic review of 442 articles of patient-reported outcome measures developed and validated for patients undergoing surgical and non-surgical facial aesthetic procedures, identified the Derriford Appearance Scale as one of only 9 favourable measures (Kosowski, McCarthy et al. 2009). However, the authors also highlight the central difficulty in using this measure for facial aesthetic surgery patients given its purpose as 
a generic measure relevant to various surgical procedures. This deficiency was particularly important when analyzing the scores of patients with a mixed clinical pattern of lipodystrophy. Although patients may have been satisfied with treatment for facial lipoatrophy, they had ongoing concerns regarding body fat distribution. Indeed, patients may become more dissatisfied with other body areas following successful treatment to the face which was previously their primary concern. Thus, improvement in concern over facial appearance may not be reflected in the DAS scores if this is the case. In retrospect, an additional measurement scale to grade facial appearance and satisfaction would have been useful in this study. However, valid, reliable and responsive instruments to measure outcomes following facial aesthetic procedures are lacking. One review of 442 articles and 47 patient-reported outcome measures for assessing facial appearance after cosmetic procedures found only 9 measures which adhered to international guidelines for the development of validation of health outcome questionnaires (Kosowski, McCarthy et al. 2009). Most of these measures are tailored to a specific procedure such as the Rhinoplasty Outcomes Evaluation or Facial Lines Treatment Satisfaction so could not be adopted in this study. Only one of the identified measures, the Facial Appearance Sorting Test, may have been appropriate for the study. This is a card-sorting test where patients are asked to rank their own personal appearance on a spectrum of attractiveness. The instrument was able to detect an improvement in appearance and psychiatric symptomology in a prospective study of 22 patients accepted for rhinoplasty. However, psychometric analysis of the test was not performed and availability of reference data are lacking.

In addition, there are some important elements lacking in all of these measures which focus only on appearance outcome. Namely, procedure-related symptoms and procedural satisfaction are not addressed. Clearly issues such as facial pain, erythema and swelling or the impact of complications on quality of life are relevant. In this study, 
an attempt to address some of these issues was made by specifically asking about procedure-related symptoms and complication during clinical follow-up. However, this was not quantified by formal means. In addition, patient satisfaction was assessed using a rating scale devised for the study although there were weaknesses with this measure which are discussed in chapter 2.

The authors of another comprehensive review of aesthetic surgery outcome measures recommend the use of a generic, utility-based quality of life instrument in conjunction with specific quality of life measures such as the DAS (Ching, Thoma et al. 2003). The reason for this is that condition-specific measures such as the DAS, which are specifically tailored to the cosmetic surgery population, cannot be used to compare different health states. In planning the study, consideration was given to use of a general quality of life scale such as the Health Measurement Questionnaire or Short Form 36 but these instruments were rejected on the basis that they may not be able to detect significant change in patients undergoing procedures predominantly cosmetic in nature. In retrospect, the inclusion of a general scale, in addition to a body image specific scale, may have been advantageous to the study. Such a scale may have better reflected the multidomain construct which defines quality of life including physical, social, psychological, emotional and spiritual domains. This may have been particularly useful to measure the psychological impact of HIV infection, especially when comparing the cohort group with controls. Interestingly, no difference was noted in psychological outcome between the HIV non-LD group and the MSM group. When interpreting this finding, consideration should be given as to whether the chosen instruments adequately assessed the relevant psychological issues. The rationale for the inclusion of only 2 short questionnaires was to minimize time in the belief that this would be more acceptable to the patient and encourage completion. However, the measures may not have adequately addressed the psychological issues pertaining to the 
HIV population. The qualitative interviews performed prior to this study were helpful to identify psychological issues specific to the HIV LD cohort and to determine whether these issues were adequately assessed using the selected psychological scales. Although, the questionnaires were found to be relevant and acceptable to patients during informal interviews prior to the study, only a small number of patients were involved. Therefore, the opinions of this group and problems experienced may not represent the HIV population as a whole. However, the interviews created an environment for patients to provide and honest account of the impact of HIV on various aspects of life such as relationships, work, and social activities. Most importantly, the effect of disfigurement on body image perception was uncovered, and the stigmatization of this particular condition was emphasized. Thus, the importance of incorporating psychological and quality of life measures to evaluate treatment outcome was confirmed. Some additional insight into the multiple factors affecting adjustment to disfigurement was revealed. For example, the importance of a stable relationship in acceptance of body image was emphasized by some patients. Indeed, these are also factors that have been demonstrated to affect adjustment to disfigurement in clinical research. Tebble showed that psychological sequelae of facial lacerations were influenced by external factors such as social support and indicated that patients in longterm relationships are less concerned about their appearance (Tebble, Thomas et al. 2004). Patient assessment of the selected psychological scales for the main study was also useful. On the whole, the content of the scales was satisfactory, although a small number of items from the DAS-24 were felt to be less appropriate by some patients. For example, 'How distressed do you get when you go to the beach?' if patients never go to the beach, or 'How distressed do you get whilst playing sports?' if the patient doesn't play sports. However, after communication with the author of the DAS 24, it 
was felt that the content should not be altered, as this would affect the scoring and validity of the scale.

\subsubsection{Comparison of study results with published clinical trials using assessment tools}

The Derriford Appearance Scale (DAS-59) and short form (DAS-24) have been used as psychological measures in several studies of different patient groups. One of the largest studies by Rumsey et al reported the extent of psychosocial needs of outpatients attending for treatment of a wide range of disfiguring conditions (Rumsey, Clarke et al. 2004). Data for the DAS-24 and HADS was collected from 15 outpatient clinics in a cross-sectional survey of 458 patients. The scores for the DAS-24 were highest in patients attending two general plastics clinics for elective procedures and in patients with tattoos (mean scores 49, 52 and 48 respectively). Scores were lowest in patients attending burns, hands and eye clinics (mean scores 32, 36 and 37 respectively). Unfortunately the results are not sub-divided according to age or gender, so it is difficult to compare directly with the HIV LD cohort. However, mean scores for HIVLD men (55) and HIV-LD females (64) appear to be higher than all of the other patient groups studied. The main drawback of Rumsey's study is the cross-sectional design. Apart from the general plastics clinics, where all of the patients are seeking elective plastic surgery, the stage of treatment for patients attending the other clinics is undisclosed. Thus, some patients may have already received treatment for disfigurement, resulting in a lower score. Also, within the general plastics groups, no information on the body areas of concern or types of procedures sought is given. Therefore, no inferences about the causality of these associations are possible. Rumsey's study also included a semi-structured interview which highlighted the importance of social support as a factor associated with reduced impact of appearance concerns on lifestyle, and reduction in perceived worry and noticeability of the 
disfigurement. This was also a factor associated with reduced concern over appearance in HIV LD patients which emerged during the semi-structured interviews in Study 1. According to Rumsey, the implication of this finding could be instrumental in rehabilitation of social difficulties relating to disfigurement by inclusion of relatives in graded exposure techniques.

Jackson et al have also utilised the DAS-24 and HADS to evaluate the psychosocial benefits of corrective surgery for adults with strabismus (Jackson, Harrad et al. 2006). A cohort of 46 patients completed the psychological measures preoperatively and 3 months postoperatively following treatment for strabismus. There was a significant reduction in mean DAS-24 scores from 40.2 preoperatively to 30.8 postoperatively. The preoperative score for this group as a whole were lower than the HIV LD group. However, the results of the strabismus group are not subdivided according to age or gender, so direct comparison with the HIV LD group is difficult. Another drawback of the study is the lack of long-term follow up data.

The DAS-24 has also been used to study appearance issues in patients following surgery for oral and oro-pharyngeal squamous cell carcinoma (Katre, Johnson et al. 2008). A postal questionnaire was sent to disease free patients identified over a 13-year period with a $66 \%$ response rate ( 252 patients). The study provides useful data for mean DAS-24 scores according to age and sex. In the 31-60 years group, males scored 32 and females scores 34 (lower than reference clinical population values). Scores in the 61 year and above age group were 26 for both males and females. However, the main disadvantage of this study is the cross-sectional design and lack of preoperative DAS-24 data to compare with other groups such as the HIV LD cohort. The study also assessed the correlation between various clinical factors with appearance. Age (younger patients), $\mathrm{T}$ stage 3 and 4 and adjuvant radiotherapy were key factors in patients reporting problems with appearance. Thus, the relatively low DAS-24 scores in this 
cohort may be influenced by age (mean 66 years) and the fact that the study is weighed towards longer-term disease free survivors. The authors also speculate that head and neck cancer patients may have fewer pre-treatment appearance concerns compared to published norms due to factors such as lower levels of self care and higher consumption of alcohol and nicotine products. Interestingly, this study also utilised the University of Washington quality of life questionnaire and found that appearance was less of a concern than other oral functional issues such as saliva, swallowing, speech and chewing. Therefore, although appearance was an important issue, it was overall a relatively lesser issue compared to function in this patient group.

The 59-item Derriford Appearance Scale was utilised as a measurement tool in Ong's study of 100 patients with HIV lipodystrophy undergoing treatment with polylactic acid (Ong, Clarke et al. 2009). The study provides useful reference data for male patients with HIV lipodystrophy at baseline and up to 24 months following treatment. There is a paucity of published reference DAS-59 data for female patients with HIV lipodystrophy. The DAS-59 comprises 6 subscales examining general selfconsciousness, social self-consciousness, self-consciousness of body and sexual selfconsciousness, negative self-consciousness, self-consciousness of facial appearance, physical distress and dysfunction as well as a whole scale score. Ong reports a statistically significant improvement in all measures of well being 6 months following treatment except for physical distress. However, at the 24-month review, all measures of well being except for social self-consciousness and physical distress returned to baseline values which Ong attributes to anticipatory anxiety over recurrence of lipodystrophy. In general, the scores provided in Ong's study are considerably higher than published studies utilising the DAS-59 in other patient groups. Litner et al utilised the scale as an outcome measure in patients undergoing cosmetic facial or nasal plastic surgery (Litner, Rotenberg et al. 2008). The overall score (81.2) was less than the HIV 
lipodystrophy group (122.4) in Ong's study. Interestingly, this study demonstrated elevated baseline levels of dysfunction for males compared with females, especially those males undergoing rhinoplasty. However, the sample size of male patients was relatively small (only $12 \%$ of the 93 patients included in the study were male). The study reports significant declines from baseline postoperatively for the full scale and for all subscales and the authors conclude that quality of life is enhanced by facial cosmetic surgery. However, only 3 months post-operative data was collected. It would be very interesting to investigate long-term psychological outcomes in patients undergoing cosmetic facial procedures, especially given the tendency for scores to return to baseline measures in the HIV lipodystrophy groups studied.

The psychological impact of minor facial lacerations has also been investigated using the DAS-59, although only 2 of the 6 subscales were selected: general selfconsciousness (GSC) and social self-consciousness (SSC) (Tebble, Thomas et al. 2004). For males aged 31-60 years, scores were 24.5 for GSC and 22.3 for the SSC. These results are lower than the HIV lipodystrophy group in Ong's study (GSC 29.2 and SSC 35.6) but higher than the general population reference values. Follow-up data collected 6 months after injury demonstrated an improvement in GSC but not for SSC, highlighting the longer term psychological consequences of even minor facial lacerations (Tebble, Adams et al. 2006).

One further study of quality of life after breast reduction utilised the DAS-59 as a measurement tool (Hermans, Boeckx et al. 2005). Unfortunately the scores are not presented in the paper therefore comparison with other patient groups is not possible. However, the authors report that insecurity, pain, shame and unattractiveness were significantly scored higher in the non-operated group.

HADS data from Rumsey's cross-sectional study of 15 outpatient clinics is useful for comparison with HIV LD patients (Rumsey, Clarke et al. 2004). Mean anxiety scores 
were greatest in general plastics (17) and rhinoplasty (12) clinics, although the mean score across all clinics was 7.9. Thus, mean anxiety scores were higher for HIV LD males (9.8) and females (9.9) compared to the mean score of all the clinics but lower than some of the individual clinics highlighted. The mean depression score across all clinics was 5.2, which was also lower than HIV LD males (7.6) and females (8.2). Similarly high scores were seen for depression in the general plastics clinic (16.4) compared to other clinics and the HIV LD groups. The explanation for the high anxiety and depression scores in the general plastics group may be related to the fact that this group are selectively more concerned with appearance as they are seeking treatment for a perceived disfigurement compared to other groups who may have already received treatment or be attending clinics for different reasons. Direct comparison with the HIV LD group is difficult as the study does not subdivide the general plastics clinic scores into age, sex or type of disfigurement. In addition, there are only 11 patients in the general plastics group studied which may be an additional source of bias. Compared to a group of patients undergoing corrective treatment for strabismus (Jackson, Harrad et al. 2006), the preoperative mean anxiety (6.4) and depression (3.7) scores were lower than the HIV LD group.

\subsubsection{Psychological outcome in HIV LD treatment group}

\subsubsection{Data completion}

Overall completion of psychological outcome data (62\%) was greater than for 3-D volume data (35\%). Repeat psychological measures were posted to patients who did not attend follow-up and this accounts for the higher completion rate. The drop-out rate was greatest in the Newfill group with only $40 \%$ completing follow-up compared to $81 \%$ in the Bio-alcamid group and $75 \%$ in the fat group. As discussed in the volume study chapter, the weaknesses of this study relate to the small numbers in each 
treatment group, confounded by incomplete follow-up data and lack of randomization. Therefore, several sources of bias exist which makes comparison between groups difficult. When considering patient motivation for completing follow-up, it was observed that those patients who attended were more likely to discuss an aesthetic concern or complication of treatment. Thus, it is possible that those patients who fail to attend are satisfied with the surgical outcome. This is an important source of bias when interpreting the psychological data. The results may be skewed, representing patients who are less satisfied with treatment. Furthermore, the potential for secondary gain must be considered. Some patients who desired re-treatment or revision procedures may have score more highly in the belief that this measure would secure further treatment using limited NHS funding. Indeed, many patients were denied treatment before funding was made available prior to the study. Another difficulty encountered was analysis of psychological data in patients who underwent revision procedures during the trial follow-up period. For these patients, completion of psychological data was continued at the defined follow-up intervals following the primary procedure. This was decided on the basis that re-commencing the follow-up period from baseline in every patient who underwent revision would further fragment the results within the small treatment groups and thus complicate statistical analysis. In addition, it would further extend the duration of the trial and most likely result in further incomplete data. However, the decision to complete the psychological data according to the follow-up period after the initial procedure introduces further bias. Repeat measures of psychological outcome in patients who were re-treated are more likely to be positively or negatively influenced by the intervention. Therefore, no inferences can be drawn about the long-term outcome of the primary procedure. The high number of revision procedures and complications with Bio-alcamid was not anticipated prior to the study. The emergence of these problems during the study created a dilemma: although 
adherence to the follow-up protocol prior to re-treatment would benefit the statistical analysis, this had to be weighed against duty of care to the patient. Unfortunately, those patients who were dissatisfied with the surgical result or developed a complication required earlier intervention, particularly as treatment was carried out using NHS resources.

\subsubsection{DAS and HADS scores}

Given the difficulty of comparison between groups described, it may be more useful to evaluate the HIV LD cohort as a whole when considering whether treatment intervention for HIV LD is associated with an improvement in psychological outcomes. In this way, patients provide their own control group, overcoming some of the additional weaknesses of non-randomization to treatment groups. Overall results showed a significant improvement in DAS and HADS-D scores following treatment. Although some increase in scores for both scales was demonstrated at 12 months, values were still significantly different compared to baseline. This study therefore supports the hypothesis that treatment for HIV LD can be associated with improvement in body image perception and psychological distress. However, the conclusions that can be drawn from analysis of individual treatment groups are less reliable and should be interpreted with caution as follows.

Despite differences in baseline clinical grading between treatment groups, psychological measures demonstrated no difference in either DAS or HADS scores between the treatment groups at baseline or at follow-up. Improvements in DAS scores compared to baseline were seen for all 3 treatments, although results only reached significance in the Bio-alcamid and Newfill groups. This may be related to the relatively small sample size in the fat group. However, 11 out of 12 patients (92\%) in the fat group had a mixed morphological pattern of lipodystrophy. Thus, treatment for facial lipoatrophy may result in some improvement in body image perception but 
patients may also have ongoing concerns regarding body fat distribution.

Unfortunately, this is not reflected in the DAS scores as the scale is not specific to facial lipoatrophy and reflects overall quality of life related to body image. The graphs also demonstrate a small increase in DAS scores (although not significant) for Newfill at 12 months. This is a finding replicated in the study by Ong et al, which demonstrated a return of DAS and HADS-A scores to baseline values between 12 and 24 months follow-up. The explanation for this may relate to anticipatory anxiety. Patients are aware that the effects of Newfill are temporary and may become increasingly worried about the recurrence of lipoatrophy. As a consequence, patients may scrutinize their face and develop self-perceived changes in treated areas, despite evidence of ongoing 3-D volume augmentation. However, current literature demonstrates high chronicity and recurrence of major anxiety disorders following treatment intervention. Although various treatment modalities including medication, cognitive therapy etc may lead to acute symptom reduction, maintenance in the long-term may not be sustained.

Therefore, this pattern of 'bounce back' in anxiety symptoms may be echoed following plastic surgery interventions in patients exhibiting anxiety trait.

The results for HADS-A and HADS-D demonstrate a similar pattern for each treatment group, with initial improvements in scores at 2 or 6 months, followed by an increase in scores at 12 months. The bounce back phenomena may account for the recurrence of symptoms following treatment, as anxiety about other issues returns. Some issues, particular to each treatment group, may also be postulated to contribute to symptom recurrence. A significant improvement in both HADS-A and HADS-D scores was seen in the Bio-alcamid group at 2 months and may represent a short-term effect following treatment in this group with the most severe facial lipatrophy at baseline. However, the high rate of revision procedures and subsequent complications with Bio-alcamid may account for a return of anxiety and depression in this group. Interestingly, the DAS 
scores remain significantly improved throughout follow-up. In the fat group, improvements in HADS-A and HADS-D reach significance by 6 months. Scores then increase at 12 months, although do not reach baseline values. This pattern may be related to fat resorption and loss of facial volume in this group (although no statistical correlation between facial volume and psychological measures was found).

Alternatively, the mixed morphology explanation described for the DAS results and ongoing dissatisfaction with body image may be relevant. The changes in HADS-A and HADS-D scores are smallest in the Newfill group and do not reach significance at any follow-up interval. One possible explanation may relate to the mechanism of action of Newfill which leads to a subtle and gradual volume augmentation. Therefore, the psychological impact of a delayed treatment effect may not be so evident compared to the immediate post-operative effect of surgical treatments such as fat transfer and Bioalcamid. However, no statistical difference was demonstrated for HADS-A and HADSD scores between Newfill and the other treatment groups. This is a finding also replicated in 2 prospective, comparative studies of fat transfer, polylactic acid and polyalcrylamide gel in the treatment of HIV lipodystrophy. The Medical Outcomes Study -HIV questionnaire was utilised in one study to evaluate psychological outcome up to 48 weeks following treatment (Negredo, Higueras et al. 2006). Significant improvements in scores were demonstrated in all 3 treatment groups compared to baseline but no difference in outcome between treatment groups was seen. However, one major limitation of this scale is that it does not assess the aesthetic appearance of the face. In another study by Guaraldi et al, psychological outcome measures included the Adult AIDS Clinical Trial Group Assessment of Body Change and Distress $(\mathrm{ABCD})$ questionnaire and a non-validated visual analogue scale to assess Facial Aesthetic Satisfaction (Guaraldi, Orlando et al. 2005). Improvements in both measures were found in all treatment groups at 24 weeks compared to baseline although no 
statistically significant differences were noted between the 3 interventional arms.

Therefore, neither this study nor any published comparative studies have been able to demonstrate a psychological advantage of one type of treatment for facial lipoatrophy. In this study, no correlation between facial volume with DAS or HADS was found. This lack of correlation is consistent with previous studies which have demonstrated a dissociation between physical deformity and psychosocial distress (Ong, Clarke et al. 2007).

\subsubsection{Correlation between psychological outcome, facial volume and clinical grading}

Interestingly, there was a relationship observed between patient grading of severity and all psychological outcomes: grade 1 lipoatrophy was associated with the lowest scores; grade 2 lipoatrophy was associated with intermediate scores and grade 3 lipoatrophy was associated with the highest scores. A much weaker relationship was seen between physician clinical grading and psychological scores. Intuitively, the belief that more severe disfigurement is associated with greater psychological distress is prevalent. However, the majority of published literature on disfigurement reports a lack of relationship between severity of disfigurement and resulting distress (Bryant and Harvey 1996; Bisson and Shepherd 1997). Research from Changing Faces (a UK-based self-help organization) has shown that a 'small mark' can lead to as many difficulties as a major disfigurement (Smith 2000). McGrouther has also reported that the impact of an injury is not proportional to the severity of the disfigurement but that other psychological factors are involved (McGrouther 1997). It is suggested that how a person reacts to a change in appearance is important rather than the change itself (Tebble, Adams et al. 2006). Also, Moss et al propose that the perceived severity of a condition provides the best predictor of distress (Moss 2005). This is an important factor to consider when assessing patients with facial disfigurements and is particularly 
relevant to patients with HIV lipodystrophy who feel stigmatized by their condition. Thus, in this study, increased psychological scores were demonstrated in patients who perceived their faces to be severely affected by lipodystrophy. This was despite physician grading being less severe for many patients. This is also an important consideration given that the basis for reconstructive surgery is predicted on the assumption that improved appearance will lead to improved psychological functioning and better quality of life. Pruzinsky has suggested that the impact of surgery may involve a complex interplay between physical, social and psychological variables, which makes it difficult to predict who will benefit from surgery (Pruzinsky 2002). Ong et al have demonstrated dissociation between subjective and objective measures of appearance during treatment for facial lipoatrophy, with only subjective assessment predicting psychological outcomes (Ong, Clarke et al. 2007). Although the results of this study suggest that treatment for HIV-associated facial lipoatrophy is associated with improvements in measured psychological outcome (for DAS-24 measures and depression), the relationship predicting severity of lipoatrophy and resultant distress (at baseline and following treatment intervention) is less well defined. Indeed, this study also demonstrated no correlation between change in facial volume following treatment and psychological outcome. Assuming that greater volume change was required to achieve restoration of facial volume in patients with greater severity of facial lipoatrophy, this finding supports the body of literature on treatment for disfigurement. Therefore, treatment of more severe clinical grade of lipoatrophy is not associated with greater improvement in psychological outcome. Moss proposes that the change in perceiving oneself as a member of a stigmatized group to a member of a 'normal' group is a crucial factor. This may also provide an explanation for discrepancy in clinical outcome and patient satisfaction, where patients may be satisfied with a less 
than optimum surgical result or dissatisfied with a good surgical result which was also found in this study.

The lack of correlation between objective 3-D data and patient reported outcomes in this and other studies calls also calls into question the usefulness of 3-D data for measuring the success of aesthetic procedures. Whilst many surgeons strive to introduce objective data for analysis of aesthetic outcome, it is unknown whether these measurements correlate with beneficial patient outcomes. In addition, there is likely to be little consensus between surgeons in the types of measurements that are considered important. This finding was also highlighted in a study by Meier et al who performed 3-D measurements of facial volume following fat grafting for facial rejuvenation in 33 patients (Meier, Glasgold et al. 2009). Patient satisfaction remained high despite only $31 \%$ of injected volume remaining at follow-up. Thus, the small measured volume change could be considered a technical failure using objective data but was still associated with a successful aesthetic outcome as rated by the patient.

A significant difference between patient and physician grading of facial lipoatrophy was demonstrated (62.8\% agreement). This may represent greater perceived severity of facial lipoatrophy by the patient due to social stigma. Alternatively, bias relating to surgeon grading may be present as scoring was performed by a single surgeon. Another drawback of the study is that clinical grading was measured at baseline only. In one study, clinical grading by the surgeon has been used as an outcome measure to evaluate Newfill treatment for HIV lipodsytrophy (Ong, Clarke et al. 2009). Interestingly, an increase in clinical grade by the examining doctor was seen at the end of the 24 month follow-up period, despite no statistically significant difference in mean 3-D laser scan volume measurements. The authors suggest a clinical bias towards placing a patient who may be of borderline grade in a worse clinical grade particularly if a change is perceived by the patient. Therefore, clinical grading by a surgeon (which may be 
perceived as a more objective measure than patient grading) is a subjective and unreliable outcome measure. However, if clinical grading is utilized, there may be an argument in favour of patient grading given that the success of aesthetic surgery is driven by the patient's perception of improvement in appearance. Indeed, Ong et al also concluded that clinical grading was more sensitive than laser scanning in predicting change in psychological functioning.

\subsection{Conclusions}

The case-control study has shown significantly higher levels of concern over body image and depression in patients with HIV associated lipoatrophy compared to HIVseropositive patients without lipodstrophy and HIV-seronegative MSM control groups. Treatment for HIV facial lipoatrophy was associated with a significant improvement in appearance related concern and depression for the cohort as a whole.

In addition, a lack of correlation between psychological measures, facial volume and clinical grading was seen. 


\section{Chapter 5. Discussion}

\subsection{Limitations of the case control study}

The limitations relating to the design of this case-control study include the potential for sampling bias and differential measurement bias. Sampling bias exists in the selection of the case group as the cases in this study may not represent psychological morbidity in patients with HIV lipodystrophy in general but rather those who present for surgical treatment. It is possible that those patients who desire surgical correction of lipodystrophy have greater concern over body image and associated psychological morbidity than those patients who do not wish treatment. Alternatively, those patients who are eligible for surgery may represent a healthier group of cases with wellcontrolled HIV parameters. Thus, it is possible that increased levels of psychological morbidity exist in HIV patients who do not present for surgical treatment due to other health or social concerns. Therefore, the design of the study could have included another control group of HIV patients with lipodystrophy who are not undergoing surgical treatment. However, the sample size of this group would be very small as the majority of patients with HIV lipodystrophy in Glasgow and Edinburgh were included in the trial or have previously undergone treatment for lipodystrophy. Sampling bias may also exist in selection of control groups. Clinic-based controls were used in this study mainly for convenience. This has the potential advantage of compensating for the potential selection bias of obtaining cases from a clinic. However, health concerns underlying clinic attendance in either control group may be positively or negatively associated with the risk factor under investigation. When assessing eligibility for the trial, patients with known psychiatric illness were excluded. However, increased anxiety associated with clinic attendance may influence results, and psychological morbidity may not be accurately represented in the MSM or non-LD HIV seropositive 
groups. A population-based sample is a measure that could have been adopted in this study. For example, a disease registry could have been used to obtain a representative sample from the general population in the area with HIV infection. However, a clinic setting was considered to encourage greater response to questionnaire completion. In addition, bias from the individuals choosing to respond may exist. Indeed, another control group of healthy subjects from the general population within the area was considered. When designing the study, this group was not considered to provide a good match to the case group with respect to confounding variables such as sexual orientation. However, following analysis of the demographic mix recruited to the case group, a local, general population sample may have been a useful addition to the study. The DAS-24 reference tables provide very useful data on both general and clinical populations which were used for comparison in this study. However, the sample of reference participants may not match the Glasgow or Edinburgh population.

Matching of control groups is another strategy to avoid sampling bias. As discussed in the relevant chapters, control groups were well matched with regards to some predictor variables such as HIV parameters but less well matched with regards to age, risk factors and ethnicity. Formal matching of controls with regards to these predictor variables was not carried out and is a limitation of the study. Patients in the case group were recruited over a 2-year interval and analysis of demographics was not possible until the end of this period. Due to time constraints, participants in the control groups were recruited simultaneously. Therefore, matching of variables such as age, sex ethnicity etc in the control groups was difficult as the demographic data for the case group was not available.

The second particular weakness of case-based studies is differential measurement bias. The method of measurement of psychological morbidity in the control groups is subject to bias as the psychological questionnaires were carried out at only one time interval. 
Performing repeat outcome measures in controls and obtaining average values may have better represented psychological morbidity in these groups over the same time interval as values obtained for cases. One other variable to consider when comparing cases with controls is potential bias relating to the strong desire for surgical treatment in the HIV LD group and how that may influence psychological scoring. Some HIV LD patients were previously denied treatment due to lack of NHS resources for aesthetic procedures. Therefore, patients may exaggerate the psychological impact of lipodystrophy and select high scores in the outcome measures in order to secure treatment. This is a difficult aspect of bias to compensate for but is worth discussion. The use of blinding in case-control studies is a strategy used to avoid bias in measuring risk factors. However, this was an impractical solution in this study as the morphological nature of HIV lipodystrophy was evident to the observer and the patients were aware of the treatment intervention. So, neither the observer nor the specific risk factor being studied could be blinded.

Following treatment for HIV lipodystrophy, there was a significant improvement in DAS-24 and HADS-D scores, to a level almost comparable with the HIV non-LD and MSM groups at 2 months. Thus treatment for HIV LD may go a long way towards restoring psychological morbidity to baseline values for HIV non-LD patients. However, longer-term follow up at 12 months demonstrated some return of psychological symptoms. This study provides comparable data for this patient group, which are not represented in the published norm tables.

\subsection{Limitations of the prospective study}

There are several limitations of the 3-D volume and psychological studies relating to the observational design. The inference that an association represents a cause-effect relation in an observational study is subject to both spurious associations and real 
associations that do not represent cause-effect, especially those due to confounding. To rule out spurious associations due to chance, calculation of sample size is important. In this study, calculations of sample size proved difficult due to the lack of published studies relating to 3-D stereophotogrammic facial volume change or data for the psychological scales chosen in HIV-lipodystrophy patients. Referral numbers of patients with HIV lipodystrophy to the plastic surgery units in Edinburgh and Glasgow in the 2-year interval prior to the trial was considered when estimating the size of patient group that could be recruited. Thus, the sample size of 50 HIV lipodystrophy patients was chosen to allow the study to be completed within a reasonable timescale whilst providing useful information for future studies. However, lack of statistical calculation of sample size is a weakness of this study.

Spurious associations due to bias must also be considered in this type of study and strategies to minimise these systematic errors in both the design and analysis phase of the research must be performed. Three concerns relate to the design phase. Firstly, does the sample of study subjects represent the population of interest? A discussion on potential bias and representativeness of the HIV lipodystrophy cohort is provided in the case-control discussion. Secondly, does the measurement of the predictor variable sufficiently represent the predictor of interest? Difficulties in defining HIV-associated lipodystrophy exist and are explained in the introduction of this thesis. In this study, a medical history and lipodystrophy-specific examination (using the patient and physician assessment of lipodystrophy by Carr et al) was carried out to define the presence and severity of HIV-associated lipodystrophy. However, the subjective nature of this assessment is demonstrated in the poor correlation between patient and physician assessment. Patients were assessed by at least 2 physicians who agreed that the morphological appearance was related to HIV-lipodystrophy (the referring HIV physician and the lead researcher). In addition, all new referrals were also assessed by a 
consultant plastic surgeon. Thirdly, does measurement of the outcome variables sufficiently represent the outcomes of interest? In this study, the measurement of facial volume utilising the DI3D system was validated and found to be accurate and reproducible. Validated psychological measures were also selected and tested in a semi-structured interview to assess suitability of the scales to a group of patients with HIV lipodystrophy. Thus, the selected scales seemed appropriate to measure the outcome of interest when designing the study. However, some weaknesses of the scales to quantify outcome following facial aesthetic surgery emerged during the study. In addition, the complex interplay of variables influencing psychological morbidity before and after surgery, particularly in an HIV cohort, may not have been fully addressed although these variables are acknowledged and discussed in chapter 4 . There are also several potential sources of bias in the analysis phase of the study. Firstly, there were a large number of patients who did not complete follow-up, particularly in the Newfill group. In addition, some patients underwent re-treatments during the follow-up interval. A detailed discussion relating to the effect of drop-outs and re-treatments on both the 3-D volume and psychological data is provided in the relevant chapters. Secondly, the majority of patients were not randomised to either the Newfill or Bio-alcamid group. Therefore, bias exists in the selection of patients to treatment type. The 3 treatment groups were also not matched. Indeed, differences in baseline characteristics between groups were observed and the potential sources of bias when analysing the measurement outcomes have been discussed. Thus, weaknesses of the study relating to incomplete follow-up, small sample size, and lack of randomisation limit the extent of comparison that can be made between treatment groups. Therefore, conclusions from the study can only be based on observation of the cohort and caution must be exerted when drawing conclusions about the advantage of one treatment type over another. 
Finally, real associations that do not represent cause-effect, such as confounding variables must be considered in an observational study. Many of the potential confounding variables and weakness of the study have been highlighted in the relevant sections of the discussion on volume measurements and psychological outcomes. For example, progression of lipodystrophy or changes in weight may affect facial volume. During the study follow-up patients were questioned about changes to antiretroviral medication or weight. However, haematological parameters such as viral load and CD4 count were not measured throughout the follow-up interval. In addition, objective measurements of body fat such as DEXA scanning or BMI measurements were not carried out at follow-up. Confounding variables that may influence psychological outcomes such as dissatisfaction with other body areas, particularly in patients with mixed morphology, or development of complications has also been considered. Although the DAS-24 is a useful tool to measure quality of life relating to body image, perhaps an additional scale to specifically measure concern over facial appearance would have been useful to assess outcome of treatment to the face.

\subsection{Comparison of treatment results and directions for future}

\section{research}

Although conclusions relating to comparison of treatment groups must be made cautiously due to the limitations of the study, some advantages and disadvantages of each treatment were observed.

Autologous fat transfer continues to remain the preferred treatment option in those patients with sufficient donor fat due to the safety of this technique and low rate of complications. A more natural volume augmentation was achieved as the treatment replaces what is anatomically deficient. The study demonstrated a mean reduction in volume compared to baseline at 6 and 12 months post-operatively. However, the 
individual response following treatment was highly variable. Thus, the major drawback of this technique is the unpredictable nature of fat resorption, related to both graft survival of the transferred fat and ongoing lipodystrophy. Further studies with larger patient numbers and extended follow-up are required to evaluate the long-term success of fat transfer. Also, further investigation utilizing histological and biological assessment of fat graft survival is needed, particularly in patients with HIV lipodystrophy. These studies would also be useful to determine the merits of fat harvested from different donor sites in HIV lipodystrophy patients. Other disadvantages of this treatment include lack of sufficient donor fat in HIV patients and the requirement for the treatment to be performed in an operating theatre, which has both practical and financial implications. Although the treatment was associated with high patient satisfaction, the long-term improvement in psychological outcome in this patient group was questionable.

Treatment with Newfill was associated with a moderate but progressive volume augmentation throughout follow-up. However, the magnitude of volume change demonstrated between individuals was variable and was not associated with total number of treatment sessions in this study. Therefore, Newfill therapy may be insufficient to fully restore facial contour in patients with severe facial lipoatrophy. Newfill appears to be a safe and effective treatment for patients with mild to moderate facial lipoatrophy. Further assessment of 3-D volume change beyond 12 months in a larger group of patients is required to determine duration of effect. Also it would be useful to further investigate the relationship between number of treatments and change in volume to better predict treatment outcome. Treatment was associated with improved quality of life relating to overall body image assessment but minimal improvement in anxiety and depression symptomatology. 
Bio-alcamid treatment was able to achieve the greatest facial volume augmentation and a permanent result was maintained. The early follow-up results were also associated with improved psychological outcomes and high patient satisfaction. Therefore, this product appeared to be a good reconstructive option for patients with severe lipoatrophy. However, the complications that have emerged during the extended clinical follow-up of these patients have led to the discontinued use of this product as a treatment option for facial lipoatrophy.

Some patients appear to shift the goals of treatment from restoration of lipoatrophy to cosmetic enhancement. Therefore, establishing realistic patient expectations and reinforcing the goals of treatment during follow-up may be important. 


\section{CHAPTER 6 - Conclusions}

\subsection{HIV LD treatment cohort versus control groups}

This study demonstrated that patients with HIV lipodystrophy have greater distress relating to body image and depression compared to HIV-seropositive patients without lipodstrophy and HIV-seronegative MSM control groups. This study provides baseline demographic psychological data for these sub-groups of the population, which are not represented in the published norm tables. This provides useful comparative data when considering the effects of confounding variables such as HIV infection and sexual orientation on psychological outcome in patients undergoing treatment for HIV lipodystrophy. Investigation of psychological measures in a larger female population of HIV-seropositive patients with and without lipodystrophy and in a general population including non-MSM participants would be useful further research.

\subsection{Prospective study of HIV LD treatment group}

This study has provided objective, measured 3-D volume data for patients undergoing treatment for HIV facial lipoatrophy. Changes in facial volume compared to baseline were observed in all 3 treatment groups. Bio-alcamid was associated with the greatest volume change which was maintained throughout follow-up, confirming the permanent nature of this product. Measured 3-D volume augmentation was maintained in the AFT group at 2 months post-operatively, but the mean volume remaining at 6 and 12 months was $66 \%$ and $54 \%$ respectively. A progressive $3-\mathrm{D}$ volume enhancement was demonstrated following Newfill therapy (from $8.6 \mathrm{cc}$ at 2 months to $12.3 \mathrm{cc}$ at 12 months) with results showing significant change compared to baseline throughout follow-up. 
In addition, experiments to validate the DI3D system and software have demonstrated the accuracy and reproducibility of the system to measure 3-D facial volume.

The results of this study demonstrated no difference in psychological outcome between groups. Overall, treatment was associated with improved body image quality of life but only a moderate improvement in depression and a minimal effect on anxiety levels were seen. Therefore, reconstructive treatment may be associated with an improvement in appearance-related concerns but may not improve symptoms of anxiety and depression in this patient group.

No correlation between facial volume change or clinical grade of lipoatrophy and psychological outcome was demonstrated in this study which supports current evidence which also reports a lack of relationship between severity of disfigurement and resulting distress.

The short and long term complications from each treatment type have been evaluated and a resultant change in management protocol has been established, with the discontinued use of Bio-alcamid. Reported patient satisfaction was high for all 3 treatments.

In conclusion, this study has provided quantification of both objective, 3-D volume data and subjective, patient-reported outcomes for patients undergoing treatment for HIV facial lipoatrophy. However, the study highlights the discrepancy between objective measures and beneficial patient outcomes when evaluating the success of an aesthetic procedure. Whilst further development of objective tools to aid surgical planning and monitor outcome are required, there is also a need for a well-developed patient-reported outcome measure designed to measure satisfaction with facial appearance and quality of life following aesthetic procedures. This is crucial for the introduction of more evidence-based practice to the field of aesthetic surgery and in setting standards for clinical decision-making among aesthetic patients. 


\section{Bibliography}

Alijotas-Reig, J., V. Garcia-Gimenez, et al. (2008). "Delayed immune-mediated adverse effects of polyalkylimide

dermal fillers." Arch Dermatol 144: 637-642.

Alster, T. and T. West (2000). "Human-derived and new synthetic injectable materials for soft-tissue augmentation: current status and role in cosmetic surgery." Plas Recon Surg 105(7): 2515-2525.

Anderson, R. and D. Maksud (1994). "Psychological adjustments to reconstructive surgery." Nursing Clinics of North America 29(4): 711-724.

Arridge, S., J. P. Moss, et al. (1985). "Three dimensional digitization of the face and skull." Journal of Maxillofacial Surgery 13(3): 136-143.

Aung, S. C., R. C. Ngim, et al. (1995). "Evaluation of the laser scanner as a surface measuring tool and its accuracy compared with direct facial anthropometric measurements." British Journal of Plastic Surgery 48(8): 551-558.

Aylard, P., J. Gooding, et al. (1987). "A validation study of 3 anxiety and depression self-assessment scales." J of Psychosomatic Research 31: 261-268.

Ayoub, A., A. Garrahy, et al. (2003). "Validation of a vision-based, three-dimensional facial imaging system." Cleft Palate-Craniofacial Journal 40(5): 523-529.

Behrens, G. and R. Schmidt (2003) "The lipodystrophy syndrome." HIV Medicine 263283.

Benn, P., C. Ruff, et al. (2003). "Overcoming subjectivity in assessing facial lipoatrophy: is there a role for three-dimensional laser scans?" HIV Med. 4(4): 325331.

Berkowitz, S. and J. Cuzzi (1977). "Biostereometric analysis of surgically corrected abnormal faces." American Journal of Orthodontics 72(5): 526-538.

Billings, E., Jr. and J. W. May, Jr. (1989). "Historical review and present status of free fat graft autotransplantation in plastic and reconstructive surgery." Plastic \& Reconstructive Surgery 83(2): 368-381.

Bisson, J. and J. Shepherd (1997). "Psychological sequelae of facial trauma." J of Trauma 43: 496-500.

Bjelland, I., A. Dahl, et al. (2003). "The validity of the Hospital Anxiety and Depression Scale: an updated literature review." Health Qual Life Outcomes 1: 14. 
Borelli, C., C. Kunte, et al. (2005). "Deep subcutaneous application of poly-L-lactic acid as a filler for facial lipoatrophy in HIV-infected patients." Skin Pharmacology \& Physiology 18(6): 273-278.

Bowskill, J., C. Baldock, et al. (1997). "Measuring facial swelling using threedimensional imaging." Medical Informatics 22(2): 155-164.

Browne, R. F., S. J. Golding, et al. (2001). "The role of MRI in facial swelling due to presumed salivary gland disease." British Journal of Radiology 74(878): 127-133.

Brucker, M., S. Sati, et al. (2008). "Long-term fate of transplanted autologous fat in a novel rabbit facial model." Plastic \& Reconstructive Surgery 122(3): 749-754.

Bryant, R. and A. Harvey (1996). "Initial post-traumatic stress responses following motor-vehicle accidents." J of Trauma Stress 9: 223-234.

Burke, P. H. and F. H. Beard (1967). "Stereophotogrammetry of the face. A preliminary investigation into the accuracy of a simplified system evolved for contour mapping by photography." American Journal of Orthodontics 53(10): 769-782.

Burnouf, M., M. Buffet, et al. (2005). "Evaluation of coleman lipostructure for the treatment of facial lipoatrophy in patients with human immunodeficiency virus and parameters associated with the efficiency of this technique. ." Arch of Dermatol 141(10): 1220-1224.

Bush, K. and O. Antonyshyn (1996). "Three-dimensional facial anthropometry using a laser surface scanner: validation of the technique." Plastic \& Reconstructive Surgery 98(2): 226-235.

Carey, D., H. Wand, et al. (2005). "Evaluation of ultrasound for assessing facial lipoatrophy in a randomized, placebo-controlled trial." AIDS. 19(12): 1325-1327. Caron, M., M. Auclair, et al. (2001). "The HIV protease inhibitor indinavir impairs sterol regulatory element-binding protein-1 intranuclear localization, inhibits preadipocyte differentiation, and induces insulin resistance." Diabetes 50(6): 13781388.

Carpaneda, C. A. (1994). "Collagen alterations in adipose autografts." Aesthetic Plastic Surgery 18(1): 11-15.

Carr, A. and D. Cooper (2000). "Adverse effects of antiretroviral therapy." Lancet 356: 1423-1430.

Carr, A., M. Law, et al. (2003). "An Objective Lipodystrophy Severity Grading Scale Derived From the Lipodystrophy Case Definition Score." JAIDS Journal of Acquired Immune Deficiency Syndromes 33(5): 571-576. 
Carr, A., J. Miller, et al. (2000). "A syndrome of lipoatrophy, lactic acidaemia and liver dysfunction associated with HIV nucleoside analogue therapy: contribution to protease inhibitor-related lipodystrophy syndrome." AIDS 14(3): F25-F32.

Carr, A., K. Samaras, et al. (1998). "Pathogenesis of HIV-1-protease inhibitorassociated peripheral lipodystrophy, hyperlipidaemia, and insulin resistance." Lancet 351(9119): 1881-1883.

Carr, A., K. Samaras, et al. (1998). "A syndrome of peripheral lipoatrophy, hyperlipidaemia, and insulin resistance in patients receiving HIV protease inhibitors." AIDS 12: 51-58.

Carr, A., K. Samaras, et al. (1999). "Diagnosis, prediction, and natural course of HIV-1 protease-inhibitor-associated lipodystrophy, hyperlipidaemia, and diabetes mellitus: a cohort study." Lancet. 353(9170): 2093-2099.

Carr, T., T. Moss, et al. (2005). "The DAS24: A short form of the Derriford Appearance Scale (DAS59) to measure individual responses to living with problems of appearance." Br J Health Psych 10(2): 285-298.

Chajchir, J., I. Benzaquen, et al. (1990). "Fat injection." Aesth Plast Surg 14: 127. Chaturvedi, S. (1991). "Clinical irrelevance of HAD factor structure " Br J of Psychology 159: 298.

Chen, D., A. Misra, et al. (2002). "Clinical review 153: Lipodystrophy in human immunodeficiency virus-infected patients." Journal of Clinical Endocrinology \& Metabolism 87(11): 4845-4856.

Ching, S., A. Thoma, et al. (2003). "Measuring outcomes in aesthetic surgery: a comprehensive review of the literature." Plas Recon Surg 111(1): 496-480.

Coleman, S. (1997). "Facial recontouring with lipostructure. ." Clin Plast Surg 24: 347. Coleman, S. (2001). "Structural fat grafts: the ideal filler?" Clin Plas Surg 28(1): 111119.

Coleman, S. (2002). "Hand rejuvenation with structural fat grafting.

." Plast Recon Surg 110(7): 1731-1744.

Coleman, S. (2004). "Treatment of facial fat atrophy related to treatment with protease inhibitors by autologous fat injection in patients with Human Immunodeficiency Virus infection." Plas Recon Surg 114(2): 556-558.

Colins, E., C. Wagner, et al. (2000). "Psychological impact of lipodystrophy syndrome

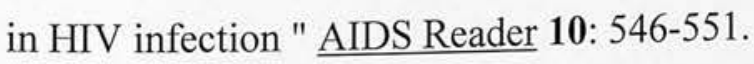


Corless, I., K. KM, et al. (2005). "Lipodystrophy-associated symptoms and medication adherence in HIV/AIDS." AIDS Patient Care STDs 19: 577-586.

Cote, H. C. F., Z. L. Brumme, et al. (2002). "Changes in mitochondrial DNA as a marker of nucleoside toxicity in HIV-infected patients." New England Journal of Medicine 346(11): 811-820.

Da Silveira, A. C., Daw, Joseph L. Jr, et al. (2003). "Craniofacial Applications of Three-Dimensional Laser Surface Scanning." Journal of Craniofacial Surgery 14(4): 449-456.

Davison, S. P., J. Timpone, Jr., et al. (2007). "Surgical algorithm for management of HIV lipodystrophy." Plastic \& Reconstructive Surgery 120(7): 1843-1858.

Dean, D., M. G. Hans, et al. (2000). "Three-dimensional Bolton-Brush Growth Study landmark data: ontogeny and sexual dimorphism of the Bolton standards cohort." $\underline{\text { Cleft }}$ Palate-Craniofacial Journal 37(2): 145-156.

Dryden, I. and K. s. e. Mardia, . (1998). Statistical Shape Analysis. Chichester, John Wiley \& Sons.

Endo, T., Y. Nakayama, et al. (1994). "Facial contour reconstruction in lipodystrophy using a double paddle dermis-fat radial forearm free flap." Ann Plas Surg 32(1): 93-96. Ersek, R. (1991). "Transplantation of purified autologous fat: A 3-year follow-up is disappointing." Plast Reconstr Surg 87: 219.

Eviatar, J., S. Silvers, et al. (2005). "Restorative treatment of HIV-associated facial lipoatrophy.." Plas Recon Surg_116(3): 29-30.

Falutz, J. (2003). "Update on HIV/HAART associated morphological and metabolic abnormalities." Forum Nutr 56: 158-162.

Farkas, L. (1994). Anthropometry of the head and face. New York., Raven Press.

Farkas, L. G., K. Hajnis, et al. (1993). "Anthropometric and anthroposcopic findings of the nasal and facial region in cleft patients before and after primary lip and palate repair." Cleft Palate-Craniofacial Journal 30(1): 1-12.

Farkas, L. G. and J. C. Posnick (1992). "Growth and development of regional units in the head and face based on anthropometric measurements,." Cleft Palate-Craniofacial Journal 29(4): 301-302.

Ferrario, V., Sforza, et al. (1997). "Preliminary evaluation of an electromagnetic threedimensional digitizer in facial anthropometry." Cleft Palate-Craniofacial Journal 35(1): 9-15. 
Ferrario, V. F., C. Sforza, et al. (1998). "Facial volume changes during normal human growth and development." Anatomical Record 250(4): 480-487.

Ferrario, V. F., C. Sforza, et al. (1998). "A three-dimensional computerized mesh diagram analysis and its application in soft tissue facial morphometry." American Journal of Orthodontics \& Dentofacial Orthopedics 114(4): 404-413.

Ferrario, V. F., C. Sforza, et al. (1994). "Comparison of soft tissue facial morphometry in children with Class I and Class II occlusions." International Journal of Adult Orthodontics \& Orthognathic Surgery 9(3): 187-194.

Fisher, D. M., L. J. Lo, et al. (1999). "Three-dimensional computed tomographic analysis of the primary nasal deformity in 3-month-old infants with complete unilateral cleft lip and palate." Plastic \& Reconstructive Surgery 103(7): 1826-1834.

Formigli, L., S. Zecchi, et al. (2004). "Bio-Alcamid: an electron microscopic study after skin implantation." Plas Recon Surg 113(3): 1104-1106.

Fourneir, P. (1986). "Microlipoexraction et microlipoinjection." Rev Chir Esthet Lang Franc 10: 36.

Ghoddousi, H., R. Edler, et al. (2007). "Comparison of three methods of facial measurement." International Journal of Oral \& Maxillofacial Surgery 36(3): 250-258. Goetzenich, A., M. Corzillius, et al. (2000). Impact of lipodystorphy on quality of life. 2000 International AIDS Conference. Durban, South Africa.

Goldan, O., I. Georgiou, et al. (2007). "Early and late complications after a nonabsorbable hydrogel polymer injection: a series of 14 patients and novel management." Dermatologic Surgery 33 Suppl 2: S199-206; discussion S206. Gooderham, M. and N. Solish (2005). "Use of hyaluronic acid for soft tissue augmentation of HIV-associated facial lipodystrophy." Dermatol Surg 31(1): 104-108. Guallar, J. P., J. M. Gallego-Escuredo, et al. (2008). "Differential gene expression indicates that 'buffalo hump' is a distinct adipose tissue disturbance in HIV-1-associated lipodystrophy." AIDS 22(5): 575-584 510.1097/QAD.1090b1013e3282f1056b1040. Guaraldi, G., D. De Fazio, et al. (2005). "Facial Lipohypertrophy in HIV-infected subjects who underwent autologous fat tissue transplantation. ." Clin Infect Diseases 40: 13-15.

Guaraldi, G., D. De Fazio, et al. (2005). "Facial lipohypertrophy in HIV-infected subjects who underwent autologous fat tissue transplantation." Clinical Infectious Diseases 40(2): e13-15. 
Guaraldi, G., G. Orlando, et al. (2005). "Comparison of three different interventions for the correction of HIV-associated facial lipoatrophy: a prospective study." Antiviral Therapy 10(6): 753-759.

Hadigan, C., K. Miller, et al. (1999). "Fasting Hyperinsulinemia and Changes in Regional Body Composition in Human Immunodeficiency Virus-Infected Women." $\underline{\mathrm{J}}$ Clin Endocrinol Metab 84(6): 1932-1937.

Hajeer MY, A. A., Millet DT (2002). "Three-dimensional imaging in orthognathic surgery: The clinical application of a new method." Int J Orthod Orthognath Surg 17(4): 1-5.

Hajeer, M. Y., Z. Mao, et al. (2005). "A new three-dimensional method of assessing facial volumetric changes after orthognathic treatment." Cleft Palate-Craniofacial Journal 42(2): 113-120.

Harris, D. and A. Carr (2001). "The Derriford Appearance Scale (DAS59): a new psychometric scale for the evaluation of patients with disfigurements and aesthetic problems of appearance." BJPS 54(3): 216-222.

Harris, D. L. and A. T. Carr (2001). "The Derriford Appearance Scale (DAS59): a new psychometric scale for the evaluation of patients with disfigurements and aesthetic problems of appearance." British Journal of Plastic Surgery 54(3): 216-222.

Harris, D. L. and A. T. Carr (2001). "Prevalence of concern about physical appearance in the general population." British Journal of Plastic Surgery 54(3): 223-226.

Hell, B. (1995). "3D sonography." International Journal of Oral \& Maxillofacial Surgery 24(1 Pt 2): 84-89.

Hermans, B. J. E., W. D. Boeckx, et al. (2005). "Quality of life after breast reduction." Annals of Plastic Surgery 55(3): 227-231.

Herrmann, C. (1997). "International experiences with the hospital anxiety and depression scale - A review of validation data and clinical results." Journal of Psychosomatic Research 42(1): 17-41.

Holberg, C., K. Schwenzer, et al. (2006). "Accuracy of facial plaster casts." Angle Orthodontist 76(4): 605-611.

Hollander, E. (1910). Munchi. med. Wschr(57): 1794.

Hudson, D., E. Lambert, et al. (1990). "Site selection for auto-transplantation: some observations." Aesthetic Plast Surg 14: 195-197.

Illouz, Y.-G. (1985). "L'Avnir de la reutilisation de la graisse apres liposuction." Rev Chir Esthet Lang Franc 1(0): 18. 
Jackson, S., R. A. Harrad, et al. (2006). "The psychosocial benefits of corrective surgery for adults with strabismus." British Journal of Ophthalmology 90(7): 883-888. James, J., A. Carruthers, et al. (2002). "HIV-associated facial lipoatrophy." Dermatol Surg 28: 979-986.

Jan, V., P. Cervera, et al. (2004). "Altered fat differentiation and adipocytokine expression are inter-related and linked to morphological changes and insulin resistance in HIV-1-infected lipodystrophic patients." Antiviral Therapy 9(4): 555-564.

Janelli, L. (1986). "The realities of body image." Journal of Geronotological Nursing 12(10): 23-27.

Jones, D., A. Carruthers, et al. (2007). "Late-appearing abscesses after injections of nonabsorbable hydrogel polymer for HIV-associated facial lipoatrophy. ." Dermatol Surg. 33: 193-198.

Jones, D., A. Carruthers, et al. (2004). "Highly purified 100-csSt silicone oil for the treatment of human immunodeficiency virus-associated facial lipoatrophy: an open pilot trial." Derm Surgery 30(10): 1279-1286.

Joy, D., R. Probert, et al. (2000). "Posttraumatic stress reactions after injury." Journal of Trauma-Injury Infection \& Critical Care 48(3): 490-494.

Karim, R., C. deLInt, et al. (2008). "Long-term effect of polyalkylimide gel injections on severity of facial lipoatrophy and quality of life of HIV-positive patients." Aesth Plast Surg 32: 873-878.

Karim, R., J. Hage, et al. (2006). "Complications of polyalkylimide 4\% injections (Bioalcamid): a report of 18 cases. ." JPRAS(59): 1409-1414.

Katre, C., I. A. Johnson, et al. (2008). "Assessment of problems with appearance, following surgery for oral and oro-pharyngeal cancer using the University of Washington appearance domain and the Derriford appearance scale." Oral Oncology 44(10): 927-934.

Kau, C. H., S. Richmond, et al. (2006). "Measuring adult facial morphology in three dimensions." Angle Orthodontist 76(5): 773-778.

Kaufman, M., J. Bradley, et al. (2007). "Autologous fat transfer national consensus survey: trends in techniques for harvest, preparation, and application, and perception of short- and long-term results. ." Plast Recon Surg 119(1): 232-331.

Kawai, T., N. Natsume, et al. (1990). "Three-dimensional analysis of facial morphology using moire stripes. Part I. Method." International Journal of Oral \& Maxillofacial Surgery 19(6): 356-358. 
Khambay, B., N. Nairn, et al. (2008). "Validation and reproducibility of a highresolution three-dimensional facial imaging system." British Journal of Oral \& Maxillofacial Surgery 46(1): 27-32.

Kobayashi, T., K. Ueda, et al. (1990). "Three-dimensional analysis of facial morphology before and after orthognathic surgery." Journal of Cranio-Maxillo-Facial Surgery 18(2): 68-73.

Koshy, C. and J. Evans (1998). "Facial contour reconstruction in localized lipodystrophy using free radial forearm adipofascial flaps. ." BJPS 51: 499-502. Kosowski, T. R., C. McCarthy, et al. (2009). "A systematic review of patient-reported outcome measures after facial cosmetic surgery and/or nonsurgical facial rejuvenation." Plastic \& Reconstructive Surgery 123(6): 1819-1827.

Krimmel, M., S. Kluba, et al. (2006). "Digital surface photogrammetry for anthropometric analysis of the cleft infant face." Cleft Palate-Craniofacial Journal 43(3): 350-355.

Lahiri, A., R. Waters, et al. (2007). "Experience with Bio-alcamid, a new soft tissue endoprosthesis." JPRAS 60: 663-667.

Lam, S. M., B. Azizzadeh, et al. (2006). "Injectable poly-L-lactic acid (Sculptra): technical considerations in soft-tissue contouring." Plastic \& Reconstructive Surgery 118(3 Suppl): 55S-63S.

Latoni, J., D. Marshall, et al. (2000). "Overgrowth of fat autotransplanted for correction of localized steroid-induced atrophy." Plast Recon Surg 106(7): 1566-1569.

Law, M., R. Puls, et al. (2006). "Evaluation of the HIV lipodystrophy case definition in a placebo-controlled, 144-week study in antiretroviral-naïve adults." Antiviral Therapy 11(2): 179-186.

Levine, B. and W. Berman (1995). "The current status of expanded polytetrafluoroethylene (Gore-tex) in facial plastic surgery." ENT journal 74(10). Lichtenstein, K., C. Wanke, et al. (2004). "Estimated prevalence of HIV-associated adipose redistribution syndrome (HARS) - abnormal abdominal fat accumulation - in HIV-infected patients." Antiviral Therapy 9(6): 33.

Lichtenstein, K., D. Ward, et al. (2001). "Clinical assessment of HIV-associated lipodystrophy in an ambulatory population." AIDS 15: 1389-1398.

Litner, J. A., B. W. Rotenberg, et al. (2008). "Impact of cosmetic facial surgery on satisfaction with appearance and quality of life." Archives of Facial Plastic Surgery 10(2): 79-83. 
Loufty, M., J. Raboud, et al. (2007). "Immediate versus delayed polyalkylimide gel injections to correct facial lipoatrophy in HIV-positive patients." $\underline{\text { AIDS } 21: ~ 1147-1155 . ~}$ Madge, S., D. Kinloch-de-Loes, et al. (1999). "Lipodystrophy in patients naive to HIV

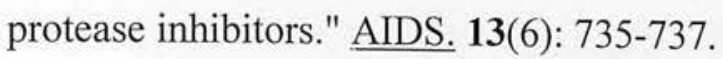

Marmot, M. G., G. D. Smith, et al. (1991). "Health inequalities among British civil servants: the Whitehall II study." Lancet 337(8754): 1387-1393.

Martinez, E., B. L, et al. (2000). "Sonographic assessment of regional fat in HIV-1infected people." Lancet. 356(9239): 1412-1413.

McCance, A. M., J. P. Moss, et al. (1992). "A three dimensional analysis of soft and hard tissue changes following bimaxillary orthognathic surgery in skeletal III patients." British Journal of Oral \& Maxillofacial Surgery 30(5): 305-312.

McCance, A. M., J. P. Moss, et al. (1992). "A three-dimensional soft tissue analysis of 16 skeletal class III patients following bimaxillary surgery." British Journal of Oral \& Maxillofacial Surgery 30(4): 221-232.

McClelland, M., B. Egbert, et al. (1997). "Evaluation of Artecoll polymethylmethacrylate implant for soft-tissue augmentation: biocompatibility and chemical characterization. ." Plas Recon Surg 100(6): 1466-1474.

McComsey, G. A. and U. A. Walker (2004). "Role of mitochondria in HIV lipoatrophy: insight into pathogenesis and potential therapies." Mitochondrion 4(2-3): 111-118.

McGrouther, D. (1997). "Facial Disfigurement. The last bastion of discrimination." The British Medical Journal 314: 991.

Meier, J. D., R. A. Glasgold, et al. (2009). "Autologous fat grafting: long-term evidence of its efficacy in midfacial rejuvenation." Archives of Facial Plastic Surgery 11(1): 2428.

Mercie, P., S. Tchamgoue, et al. (1999). "Lipodystrophy in HIV-1-infected patients." Lancet 354(9181): 867-868.

Mest, D. R. and G. Humble (2006). "Safety and efficacy of poly-L-lactic acid injections in persons with HIV-associated lipoatrophy: the US experience." Dermatologic Surgery 32(11): 1336-1345.

Michopolous, I., A. Douzenis, et al. (2008). "Hospital Anxiety and Depression Scale (HADS): validation in a Greek general hospital sample." Annals of General Psychiatry 7(4): 1744. 
Milinkovie, A. and E. Martinez (2005). "Current perspectives on HIV-associated lipodystrophy syndrome." J Antimichrobial Chemo 56: 6-9.

Miller, A., A. Carr, et al. (2003). "HIV lipodystrophy: prevalence, severity and correlates of risk in Australia." HIV Med. 4(3): 293-301.

Miller, J. (2002). "Fat hypertrophy after autologous fat transfer." Opthalm Plast \& Recon Surg 18(3): 228-231.

Moore, J. J., J. Kolaczynski, et al. (2005). "Viability of fat obtained by syringe suction lipoctomy: effects of local anaesthesia with lidocaine. ." Aesthetic Plast Surg 19: 335339.

Moss, J. P. (2006). "The use of three-dimensional imaging in orthodontics." European Journal of Orthodontics 28(5): 416-425.

Moss, T. (2005). "The relationships between objective and subjective ratings of disfigurement severity, and psychological adjustment." Body Image 2: 151-159. Moyle, G., L. Lysakova, et al. (2004). "A randomized open-label study of immediate versus delayed polylactic acid injections for the cosmetic management of facial lipoatrophy in persons with HIV infection." HIV Med. 5(2): 82-87.

Negredo, E., C. Higueras, et al. (2006). "Reconstructive treatment for antiretroviralassociated facial lipoatrophy: a prospective study comparing autologous fat and synthetic substances." AIDS Patient Care \& Stds 20(12): 829-837.

Nelson, L. and K. Stewart (2008). "Plastic surgical options for HIV-associated lipodystrophy' " JPRAS 61(4): 359-365.

Neuber, F. (1893). "Fettransplantation. ." Chir Kongr Verhandl Dtsch Ges Chir 22: 66. Nguyen, A., K. Pasyk, et al. (1990). "Comparative study of survival of autologous adipose tissue taken and transplanted by different techniques." Plast Recon Surg $\mathbf{8 5}$ : 378.

Niechajev, I. and O. Sevcuk (1994). "Long-term results of fat transplantation: clinical and histologic studies." Plastic \& Reconstructive Surgery 94(3): 496-506.

Niechajev, I. and O. Sevcuk (1994). "Long-term results of fat transplantation: clinical and histologic studies. ." Plas Recon Surg 94(3): 496-506.

O'Grady, K. F. and O. M. Antonyshyn (1999). "Facial asymmetry: three-dimensional analysis using laser surface scanning." Plastic \& Reconstructive Surgery 104(4): 928 937. 
O'Higgins, P. and N. Jones (1998). "Facial growth in Cercocebus torquatus: an application of three-dimensional geometric morphometric techniques to the study of morphological variation." Journal of Anatomy 193(Pt 2): 251-272.

Oette, M., P. Juretzko, et al. (2002). "Lipodystrophy syndrome and self-assessment of well-being and physical appearance in HIV-positive patients." AIDS Patient Care \& Stds 16(9): 413-417.

Ong, J., A. Clarke, et al. (2007). "Does severity predict distress? The relationship between subjective and objective measures of appearance and psychological adjustment, during treatment for facial lipoatrophy." Body Image 4(3): 239-248. Ong, J., A. Clarke, et al. (2009). "Objective evidence for the use of polylactic acid implants in HIV-associated facial lipoatrophy using three-dimensional surface laser scanning and psychological assessment." Journal of Plastic, Reconstructive \& Aesthetic Surgery: JPRAS 62(12): 1627-1635.

Orentreich, D. and A. Leone (2004). "A case of HIV-associated facial lipostrophy treated with 1000-cs liquid injectable silicone." 30: 548-551.

Pacini, S., M. Ruggiero, et al. (2003). "Bio-alcamid, a novel prosthetic polymer, does not interfere with morphological and functional characteristics of human skin fibroblasts. ." Plas Recon Surg 111(1): 489-491.

Pacini, S., M. Ruggiero, et al. (2002). "Bio-alcamid: a novelty for reconstructive and cosmetic surgery." Ital J Anat Embryol 107: 209-214.

Paech, V., T. Lorenzen, et al. (2002). "Gynaecomastia in HIV-infected men: association with effects of antiretroviral therapy." AIDS 16(8): 1193-1195. Partridge, J. (1993). "The psychological effects of facial disfigurement." $\underline{\mathrm{J} \text { of Wound }}$ Care 2(3): 168-171.

Peer, L. (1955). "Cell survival theory versus replacement theory." 1946). 16(3): 161168.

Peer, L. (1956). "The neglected free fat graft." Plast Reconstr Surg 18: 233-250.

Pettit, R., D. Kotler, et al. (1999). Abnormalities in HIV-associated lipodystrophy syndrome that vary by weight status. . 1st International Workshop on Adverse Drug Reactions and Lipodystrophy in HIV, San Diego.

Piliero, P., M. Hubbard, et al. (2003). "The use of ultrasonography-assisted liposuction for the treatment of human immunodeficiency virus-associated enlargement of the dorsocervical fat pad." Clin Infect Diseases 37: 1374-1377. 
Price, B. (1995). "Assessing altered body image. ." J of Psych and Mental Health

Nursing 2: 161-175.

Protopapa, C., G. Sito, et al. (2003). "Bio-alcamid in drug-induced lipodystrophy." J

Cosmetic \& Laser Ther 5: 226-230.

Pruzinsky, T. (2002). Body image adaption to reconstructive surgery for aquired disfigurement. London, Guilford Press.

Pu, L., S. Coleman, et al. (2008). "Autologous fat grafts harvested and refined by the Coleman technique: a comparative study." 122 3(932-937).

Pu, L., X. Cui, et al. (2005). "The viability of fatty tissues within adipose aspirates after conventional liposuction: a comprehensive study. ." Ann Plast Surg 54(3): 288-292.

Rabey, G. (1971). "Craniofacial morphanalysis." Proceedings of the Royal Society of Medicine 64(2): 103-111.

Ramires, P., M. Miccoli, et al. (2005). "In Vitro and In Vivo Biocompatibility of a Polyalkylimide Hydrogel for Soft Tissue Augmentation." J of Biomed Materials Research 72(2): 230-238.

Ramon, Y., F. Lucian, et al. (2007). "Preliminary experiences with Bio-alcamid in HIV facial lipoatrophy. ." Dermatology. 214: 151-154.

Ras, F., L. Habets, et al. (1996). "Quantification of facial morphology using stereophotography - a demonstration of a new concept." J Dentristy 24(5): 369-374.

Ras, F., L. L. Habets, et al. (1996). "Quantification of facial morphology using stereophotogrammetry--demonstration of a new concept." Journal of Dentistry 24(5): 369-374.

Rasse, M., G. Forkert, et al. (1991). "Stereophotogrammetry of facial soft tissue." Int J Oral Maxillofac Surg. 20(3): 163-166.

Rees, T., F. Ashley, et al. (1973). "Silicone fluid injections for facial atrophy. A ten year study." Plas Recon Surg_52(2): 118-126.

Reynolds, N., J. Neidig, et al. (2006). "Balancing disfigurement and fear of disease progression: patient perceptions of HIV body fat redistribution." AIDS Care 18(7): 663-673.

Rigotti, G., A. Marchi, et al. (2007). "Clinical treatment of radiotherapy tissue damages by lipoaspirates transplant: a healing process mediated by adipose derived stem cells." Plast Recon Surg 19(5): 1409-1422. 
Ritt, M., M. Hillebrand-Haverkort, et al. (2001). "Local treatment of facial lipodystrophy in patients receiving HIV protease inhibitor therapy." Acta Chirurgiae Plasticae 43(2): 54-56.

Rohrich, R., E. Sorokin, et al. (2004). "In search of improved fat transfer viability: a quantitative analysis of the role of centrifugation and harvest site." Plast Recon Surg 113(1): 391-395.

Ross, A. H. and R. Malhotra (2009). "Long-term orbitofacial complications of polyalkylimide 4\% (bio-alcamid)." Ophthalmic Plastic \& Reconstructive Surgery 25(5): 394-397.

Rubin, A. and S. Hoefflin (2002). "Fat purification: survival of the fittest." Plast Recon Surg 109: 1463.

Rumsey, N., A. Clarke, et al. (2004). "Altered body image: appearance-related concerns of people with visible disfigurement." Journal of Advanced Nursing 48(5): 443-453.

Saint-Marc, T., M. Partisani, et al. (2000). "Fat distribution evaluated by computed tomography and metabolic abnormalities in patients undergoing antiretroviral therapy: preliminary results of the LIPOCO* study." AIDS 14(1): 37-49.

Sattler, F. (2003). "Body habitus changes relating to lipodystrophy." Clin Infect Diseases 36: 84-90.

Savard, J., B. Laberge, et al. (1998). "Evaluating anxiety and depression in HIVinfected patients." J Pers Assess 71(3): 349-367.

Sekhar, R. V., F. Jahoor, et al. (2002). "Metabolic basis of HIV-lipodystrophy syndrome." American Journal of Physiology - Endocrinology \& Metabolism 283(2). E332-337.

Sen, P., N. Ross, et al. (2001). "Recovering maxillofacial trauma patients: the hidden problems." J Wound care 10: 53.

Serra-Renom, J. and J. Fontdevila (2004). "Treatment of facial fat atrophy related to treatment with proteast inhibitors by autologous fat injection in patients with Human Immunodeficiency Virus infection.". Plas Recon Surg 114(2): 551-555.

Serra, M., L. Oyafuso, et al. (2004). Polymethylmethacrylate (PMMA) for facial atrophy treatment: 5 years follow-up. 15th International AIDS conference, Bangkok, Thailand, . 
Singh, I. and B. Savara (1966). "Norms of size and annual increments of seven anatomical measures of maxillae in girls from three to sixteen years of age." Angle Orthod. 36(4): 312-324.

Smith, H. (2000). "Challenging Disfigurement." Community Practitioner 73(6): 637. Smith, P., W. Adams, et al. (2006). "Autologous human fat grafting: effect of harvesting and preparation on adipocyte graft survival. ." Plast Recon Surg 117(6): 1836-1844.

Sommer, B. and G. Sattler (200). "Current concepts of fat graft survival: histology of aspirated adipose tissue and review of the literature. ." Dermatol Surg 26: 1159-1166. Stauch, B., T. Baum, et al. (2004). "Treatment of Human Immunodeficiency VirusAssociated Lipodystrophy with dermafat graft transfer to the malar area. ." Plas Recon Surg 113(1): 363-370.

Stevens, W. P. (1997). "Reconstruction of three-dimensional anatomical landmark coordinates using video-based stereophotogrammetry." Journal of Anatomy 191(Pt 2): 277-284.

Talmor, M., L. Hoffman, et al. (2002). "Facial atrophy in HIV-related fat redistribution syndrome: anatomic evaluation and surgical reconstruction." Ann Plas Surg 49(1): 1111.

Tebble, N., D. Thomas, et al. (2004). "Anxiety and self-conciousness in patients with minor facial lacerations." J of Advanced Nursing 47(4): 417-426.

Tebble, N. J., R. Adams, et al. (2006). "Anxiety and self-consciousness in patients with facial lacerations one week and six months later." British Journal of Oral \& Maxillofacial Surgery 44(6): 520-525.

Ullmann, Y., O. Shoshani, et al. (2005). Dermatolog Surg 31(10): 1304-1307. Valantin, M., C. Aubron-Olivier, et al. (2003). "Polylactic acid implants (New-Fill) to correct facial lipoatrophy in HIV-infected patients: results of the open-label study VEGA." AIDS. 17(17): 2471-2477.

Valantin, M., C. Aubron-Olivier, et al. (2003). "Polylactic acid implants (Newfill) to correct facial lipoatrophy in HIV-infected patients: results of the open-label study

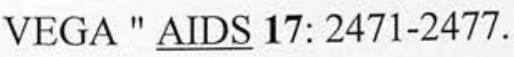

van der Wal, K. and J. Mulder (1998). "Facial contour reconstruction in partial lipodystrophy using two temporalis muscle flaps. A case report. ." Int J Oral \& Max Surg 27(1): 14-16. 
Vleggaar, D. (2006). "Soft-tissue augmentation and the role of poly-L-lactic acid." Plastic \& Reconstructive Surgery 118(3 Suppl): 46S-54S.

Vleggaar, D. and U. Bauer (2004). "Facial enhancement and the European experience with Sculptra (poly-1-lactic acid)." Journal of Drugs in Dermatology: JDD 3(5): 542547.

Walli, R., O. Herfort, et al. (1998). "Treatment with protease inhibitors associated with peripheral insulin resistance and impaired oral glucose tolerance in HIV-1-infected patients." AIDS 12(15): F167-173.

Watson, R. M., T. J. Coward, et al. (1993). "Considerations in treatment planning for implant-supported auricular prostheses." International Journal of Oral \& Maxillofacial Implants 8(6): 688-694.

Wechselberger, G., M. Sarcletti, et al. (2001). "Dermis-Fat graft for facial lipodystrophy in HIV-positive patients: is it worthwhile? ." Ann Plas Surg 47(1): 99100.

Yip, E., A. Smith, et al. (2004). "Volumetric evaluation of facial swelling utilizing a 3D range camera." International Journal of Oral \& Maxillofacial Surgery 33(2): 179182.

Yuksel, E., A. Weinfeld, et al. (2000). "Increased free fat-graft survival with the longterm, local delivery of insulin, insulin-like growth factor-1, and basic fibroblast growth factor by PLGA/PEG microspheres. ." Plast Recon Surg 105: 1712.

Zigmond, A., R. Snaith, et al. (1983). "The Hospital Anxiety and Depression Scale. ." Acta Psych Scand 67: 361-370. 
APPENDIX 1. Patient and physician assessment of lipodystrophy 


\section{APPENDIX 2. Patient Satisfaction Scales}




\section{$\underline{\text { Patient satisfaction questionnaire }}$}

1. On the following scale from 1-10, how would you rate your satisfaction with the treatment you received:

$\begin{array}{lc}\text { Completely } & \begin{array}{c}\text { Completely } \\ \text { Unsatisfied }\end{array} \\ 11 & 10\end{array}$

2. On the following scale, to what extent do you feel your appearance has improved:
No improvement
at all
Best outcome
expected

10

3. To what extent would you recommend the treatment you received to others:
I would definitely
not recommend it
I would definitely recommend it

4. How would you rate the result of your surgery compared with what you expected?

Much worse than

Much better than

I expected

I expected

10

Please circle the response that applies to you:

5. What treatments did you receive?

Fat injections Bio-alcamid injections Newfill injections Liposuction

6. Have the effects of treatment lasted? Yes No

If not, how long after surgery did you notice a return of the problem?

0-3 months $3-6$ months $6-9$ months $9-12$ months $12-18$ months

7. Did you have any complications following surgery (e.g. bleeding, bruising, wound infection? Yes No 
If you answered yes, please give details:

If you have any other comments about your surgery (good or bad) please write them in the space below.

Thank you very much for completing this questionnaire. Your help is very much appreciated! 


\section{APPENDIX 3. Linear mixed effect regression models}

\begin{tabular}{|c|c|c|c|c|c|c|c|c|c|}
\hline \multirow[t]{2}{*}{ Outcome } & \multirow[t]{2}{*}{ Visit } & \multicolumn{3}{|c|}{ LME Mean $(95 \% \mathrm{CI})$} & \multicolumn{3}{|c|}{ LME Change $(95 \% \mathrm{CI}), \mathrm{p}$} & \multicolumn{2}{|c|}{$\begin{array}{l}\text { Between-Group p- } \\
\text { value }\end{array}$} \\
\hline & & Bio-Alcamid & Fat & NewFill & Bio-Alcamid & Fat & NewFill & Mean & Change \\
\hline Volume & $\begin{array}{l}\text { Injected } \\
2 \text { Months } \\
6 \text { Months } \\
12 \text { Months }\end{array}$ & $\begin{array}{c}25.5(20.5, \\
30.5) \\
26.9(21.8, \\
32.0) \\
26.1(20.9 \\
31.4) \\
28.7(23.3, \\
34.1)\end{array}$ & $\begin{array}{c}20.3 \\
(13.9 \\
26.8) \\
16.4(9.5, \\
23.3) \\
12.9(6.2 \\
19.7) \\
11.5(4.6 \\
18.4)\end{array}$ & $\begin{array}{c}8.6(3.3, \\
13.9) \\
12.8(6.9, \\
18.8) \\
4.3(-1.9, \\
10.4)\end{array}$ & $\begin{array}{l}1.4(-1.9,4.8) \\
p=0.395 \\
0.7(-2.3,3.6) \\
p=0.651 \\
3.2(-0.1,6.6) \\
p=0.058\end{array}$ & $\begin{array}{c}-3.9(-9.0,1.1) \\
p=0.129 \\
-7.4(-11.1,- \\
3.7), p<0.001 \\
-8.8(-13.0,- \\
4.6), p<0.001\end{array}$ & - & $\begin{array}{l}p=0.219 \\
p<0.001 \\
p=0.001 \\
p<0.001\end{array}$ & $\begin{array}{l}p=0.082 \\
p=0.001 \\
p<0.001\end{array}$ \\
\hline DAS & $\begin{array}{l}\text { Baseline } \\
2 \text { Months } \\
6 \text { Months } \\
12 \text { Months }\end{array}$ & $\begin{array}{c}55.5(46.5, \\
64.5) \\
41.6(32.5, \\
50.7) \\
43.1(33.7, \\
52.5) \\
41.4(32.1, \\
50.7)\end{array}$ & $\begin{array}{c}49.6 \\
(39.3, \\
59.9) \\
41.2 \\
(30.6, \\
51.7) \\
42.8 \\
(32.3, \\
53.2) \\
42.7 \\
(32.0 \\
53.3)\end{array}$ & $\begin{array}{c}56.2(48.1, \\
64.2) \\
40.2(31.7, \\
48.8) \\
39.9(30.3, \\
49.5) \\
46.2(36.5, \\
55.9)\end{array}$ & $\begin{array}{l}-13.9(-21.5,- \\
6.4), p<0.001 \\
-12.4(-20.5,- \\
4.3), p=0.003 \\
-14.1(-21.6,- \\
6.6), p<0.001\end{array}$ & $\begin{array}{c}- \\
-8.4(-17.5, \\
0.7), \mathrm{p}=0.070 \\
-6.8(-16.1 \\
2.5), \mathrm{p}=0.150 \\
-6.9(-15.8 \\
1.9), \mathrm{p}=0.124\end{array}$ & $\begin{array}{c}-15.9(-23.1,- \\
8.7), \mathrm{p}<0.001 \\
-16.2(-24.8,- \\
7.6), \mathrm{p}<0.001 \\
-10.0(-18.4,- \\
1.5), \mathrm{p}=0.020\end{array}$ & $\begin{array}{l}p=0.585 \\
p=0.977 \\
p=0.882 \\
p=0.773\end{array}$ & $\begin{array}{l}p=0.443 \\
p=0.349 \\
p=0.468\end{array}$ \\
\hline HADS-A & $\begin{array}{l}\text { Baseline } \\
2 \text { Months } \\
6 \text { Months } \\
12 \text { Months }\end{array}$ & $\begin{array}{c}9.4(6.8, \\
11.9) \\
6.8(4.3, \\
9.4) \\
8.7(6.0, \\
11.4) \\
7.9(5.3, \\
10.5)\end{array}$ & $\begin{array}{c}9.9(7.1, \\
12.8) \\
8.3(5.3, \\
11.3) \\
7.5(4.6, \\
10.5) \\
8.9(5.9, \\
11.9)\end{array}$ & $\begin{array}{c}9.8(7.5, \\
12.1) \\
9.3(6.9, \\
11.6) \\
8.9(6.1, \\
11.7) \\
9.6(6.8, \\
12.4)\end{array}$ & $\begin{array}{c}-2.6(-4.3,-0.8) \\
p=0.005 \\
-0.7(-2.6,1.2) \\
p=0.487 \\
-1.5(-3.3,0.4) \\
p=0.125\end{array}$ & $\begin{array}{c}-1.6(-3.8,0.6) \\
p=0.155 \\
-2.4(-4.6,- \\
0.2), p=0.033 \\
-1.0(-3.3,1.2) \\
p=0.366\end{array}$ & $\begin{array}{c}-0.5(-2.2,1.2) \\
p=0.545 \\
-0.9(-3.1,1.3) \\
p=0.425 \\
-0.2(-2.5,2.0) \\
p=0.848\end{array}$ & $\begin{array}{l}p=0.954 \\
p=0.381 \\
p=0.775 \\
p=0.690\end{array}$ & $\begin{array}{l}p=0.265 \\
p=0.481 \\
p=0.705\end{array}$ \\
\hline HADS-D & $\begin{array}{l}\text { Baseline } \\
2 \text { Months } \\
6 \text { Months } \\
12 \text { Months }\end{array}$ & $\begin{array}{c}7.6(5.2, \\
10.1) \\
4.6(2.2, \\
7.1) \\
6.3(3.7, \\
8.8) \\
6.2(3.7, \\
8.7)\end{array}$ & $\begin{array}{c}5.8(3.1, \\
8.6) \\
4.2(1.3, \\
7.1) \\
3.5(0.7, \\
6.4) \\
4.7(1.8, \\
7.6)\end{array}$ & $\begin{array}{c}7.2(5.0, \\
9.4) \\
6.0(3.7, \\
8.3) \\
5.9(3.3, \\
8.6) \\
6.5(3.9, \\
9.2)\end{array}$ & $\begin{array}{c}-3.0(-4.6,-1.4) \\
p<0.001 \\
-1.3(-3.1,0.4) \\
p=0.132 \\
-1.4(-3.1,0.3) \\
p=0.100\end{array}$ & $\begin{array}{c}-1.6(-3.6,0.4) \\
p=0.118 \\
-2.3(-4.3,- \\
0.3), p=0.027 \\
-1.1(-3.1,0.9) \\
p=0.293\end{array}$ & $\begin{array}{c}- \\
-1.2(-2.8,0.4) \\
p=0.129 \\
-1.3(-3.3,0.8) \\
p=0.224 \\
-0.7(-2.7,1.4) \\
p=0.515\end{array}$ & $\begin{array}{l}p=0.616 \\
p=0.582 \\
p=0.329 \\
p=0.645\end{array}$ & $\begin{array}{l}p=0.275 \\
p=0.735 \\
p=0.858\end{array}$ \\
\hline
\end{tabular}

\section{APPENDIX 4. Derriford Appearance Scale}


DATE

OCCUPATION: Yours

Partners/Spouse's

YOUR FAMILY STATUS (please tick the option closest to your situation)

Married/Living with partner

Living alone

Living with relatives/friends 


\section{This questionnaire is concerned with how you feel about your appearance}

The first part of the scale is designed to find out if you are sensitive or self-conscious about any aspect of your appearance (even if this is not usually visible to others).

(a) Is there any aspect of your appearance (however small) that concerns you

$$
\text { at all? Yes / No }
$$

If No, please turn to the next

page If Yes, please continue:

(b) The aspect of my appearance about which I am most sensitive or self-conscious is

\section{From now on, we will refer to this aspect of your appearance as your 'feature'}

(c) The thing I don't like about my feature is

(d) If you are sensitive or concerned about any other features of your body or your appearance, please say what they are

a. 1996 . 
Instructions: The following questions are concerned with the way you feel or act. They are all_simple. Please tick the answer that applies to you. If the item does not apply to you at all, tick the N/A (not applicable option). Don't spend long on any one question.

a. How confident do you feel?

Not at all .... Slightly .... Moderately .... Extremely ....

b. How distressed do you get when you see yourself in the mirror/window? Extremely ...... Moderately.... A Little .... Not at all Distressed ....

c. My self-consciousness makes me irritable at home:

N/A ..... Never/Almost never .... Sometimes .... Often .... Almost always .

d. How hurt do you feel?

Extremely .... Moderately .... Slightly .... Not at all ....

e. At present my self-consciousness has an adverse effect on my work:

Almost always .... Often .... Sometimes .... Never/almost never ... N/A .....

f. How distressed do you get when you go to the beach?

N/A ..... Not at all .... A little .... Moderately ... Extremely ....

g. Other people mis-judge me because of my feature:

Almost always .... Often ... Sometimes... Never/almost never ...... N/A .....

h. How feminine/masculine do you feel?

Not at all .... Slightly Moderately Extremely ....

i. I am self-conscious of my feature:

N/A ... Never/Almost never .... Sometimes .... Often .... Almost always ....

j. How irritable do you feel?

Not at all Slightly .... Moderately .... Extremely ....

k. Tadopt certain gestures (e.g. folding my arms in front of other people, covering my mouth with my hand):

Never/almost never .... Sometimes .... Often .... Almost always ...

I avoid communal changing rooms:

Almost always .... Often .... Sometimes.... Never/almost never ..... N/A ..... 
m. How distressed do you get by shopping in department stores/supermarkets?

$$
\text { N/A ..... Not at all .... Slightly .... Moderately .... Extremely .... }
$$

n. How rejected do you feel?

$$
\text { Not at all .... Slightly .... Moderately .... Extremely .... }
$$

o. I avoid undressing in front of my partner:

N/A ..... Never/almost never .... Sometimes .... Often .... Almost always ....

p. How distressed do you get while playing sports/games?

Extremely .... Moderately .... Slightly .... Not at all .... N/A .....

q. I close into my shell:

Almost always .... Often .... Sometimes.... Never/Almost never

r. How distressed are you by being unable to wear your favourite clothes?

Extremely .... Moderately .... Slightly .... Not at all .... N/A .....

S. How distressed do you get when going to social events?

N/A ..... Not at all ........ Moderately .... A fair amount .... Extremely ....

t. How normal do you feel?

Not at all .... Slightly .... Moderately.... Extremely

u. At present my self-consciousness has an adverse effect on my sex life:

Almost always .... Often .... Sometimes... Never/almost never .... N/A .....

v. I avoid going out of the house:

Almost always ... Often ... Sometimes.... Never/almost never ....

w. How distressed do you get when other people make remarks about your feature?

N/A ... Notatall.... Moderately .... A fair amount .... Extremely ....

\section{x. I avoid going to pubs/restaurants:}

My feature causes me physical pain/discomfort:

Never/almost never .... Sometimes .... Often .... Almost always ....

My feature limits my physical ability to do the things I want to do:

Almost always .... Often .... Sometimes.... Never/ almost never ... 
APPENDIX 5. Hospital Anxiety \& Depression Scale 


\begin{tabular}{|c|c|c|c|c|}
\hline & $\begin{array}{c}\text { Yes } \\
\text { definitely }\end{array}$ & \begin{tabular}{c|} 
Yes \\
sometimes \\
\end{tabular} & $\begin{array}{c}\text { No, not } \\
\text { much }\end{array}$ & $\begin{array}{c}\text { No, not } \\
\text { at all }\end{array}$ \\
\hline $\begin{array}{l}\text { 1. I wake early and then sleep badly for the } \\
\text { rest of the night. }\end{array}$ & 3 & 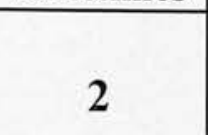 & 1 & $\mathbf{0}$ \\
\hline $\begin{array}{l}\text { 2. I get very frightened or have panic } \\
\text { feelings for apparently no reason at all. }\end{array}$ & 3 & 2 & 1 & $\mathbf{0}$ \\
\hline 3. I feel miserable and sad. & 3 & 2 & 1 & $\mathbf{0}$ \\
\hline $\begin{array}{l}\text { 4. I feel anxious when I go out of the } \\
\text { house on my own. }\end{array}$ & 3 & 2 & 1 & $\mathbf{0}$ \\
\hline 5. I have lost interest in things. & 3 & 2 & 1 & $\mathbf{0}$ \\
\hline $\begin{array}{l}\text { 6. I get palpitations, or sensations of } \\
\text { 'butterflies' in my stomach or chest. }\end{array}$ & 3 & 2 & 1 & $\mathbf{0}$ \\
\hline 7. I have a good appetite. & $\mathbf{0}$ & 1 & 2 & 3 \\
\hline 8. I feel scared or frightened. & 3 & 2 & 1 & $\mathbf{0}$ \\
\hline 9. I feel life is not worth living. & 3 & 2 & 1 & $\mathbf{0}$ \\
\hline 10. I still enjoy the things I used to. & $\mathbf{0}$ & 1 & 2 & 3 \\
\hline 11. I am restless and can't keep still. & 3 & 2 & 1 & $\mathbf{0}$ \\
\hline 12. I am more irritable than usual. & 3 & 2 & 1 & $\mathbf{0}$ \\
\hline 13. I feel as if I have slowed down. & 3 & 2 & 1 & $\mathbf{0}$ \\
\hline $\begin{array}{l}\text { 14. Worrying thoughts constantly go } \\
\text { through my mind. }\end{array}$ & 3 & 2 & 1 & 0 \\
\hline
\end{tabular}
Anxiety $2,4,6,8,11,12,14$
Depression $1,3,5,7,9,10,13$
Scoring 3, 2, 1, 0 (For items $7 \& 10$ the scoring is reversed)
GRADING: $0-7=$ Non-case $8-10=$ Borderline case $11+=$ Case 
APPENDIX 6. Reference qualitative interview questions 


\section{APPENDIX 7. Presentations}

\section{International}

\section{BAAPS UK Representative in Best of Europe}

L Nelson, K.J. Stewart . 3-D Volume analysis of facial fat transfer and comparison with subjective assessment of fat resorption' EASAPS, June 2010, Aachen

2. L Nelson, D.S. Soutar, K.J. Stewart. 'Psychological morbidity and facial volume in HIV-associated lipodystrophy: quantification of treatment outcome.' EURAPS, May 2010 Manchester

3. L Nelson, D.S. Soutar, K.J. Stewart 'Facial volumes and psychological morbidity in HIV-lipodystrophy' European Association of Societies of Aesthetic Plastic Surgery, October 2008, Madrid

4. L Nelson, D.S. Soutar, K.J. Stewart 'Facial volumes and psychological morbidity in HIV-lipodystrophy' European Academy of Facial Plastic Surgeons, September 2008, Dusseldorf

5. L Nelson, K.J. Stewart. 'Experience in the treatment of HIV lipodystrophy'. European Association of Plastic and Reconstructive Surgeons, May 2008, Madiera

6. L Nelson, K.J. Stewart. 'Experience in the treatment of HIV lipodystrophy'. European Conference of Scientists and Plastic Surgeons, September 2007, Aachen, Germany

\section{National}

1.Nelson L, D.S. Soutar, K.J. Stewart .'3-D Volume analysis of facial fat transfer and comparison with subjective assessment of fat resorption' British Association of Aesthetic Plastic Surgeons, September 2009, Cardiff $\mathbf{1}^{\text {st }}$ Prize

2. Nelson L, D.S. Soutar, K.J. Stewart. 'Quantification of treatment outcomes in HIV-associated lipodystrophy.' Thirty Year of Plastic Surgery, Friends of Canniesburn, April 2008, Glasgow

3. L Nelson, K.J.Stewart. 'Experience in the treatment of HIV lipodystrophy'. Scottish-Irish Meeting, October 2007, Aberdeen 
4.L Nelson, K.J.Stewart. 'Experience in the treatment of HIV lipodystrophy'.

British Association of Aesthetic Plastic Surgeons Sept 2006, Bath

\section{Poster presentations}

1. L. Nelson, K.J. Stewart 'Quantification of facial volume in HIVlipodystrophy' British Association of Aesthetic Plastic Surgeons, September 2008 Chester

2. L. Nelson, K.J. Stewart, A. Winters, D Clutterbuck 'Psychological Morbidity in HIV-associated lipodystrophy' British HIV Association Annual Meeting, April 2008, Belfast. 


\section{Appendix 8. Publications}

L Nelson, K.J. Stewart. Experience in the treatment of HIV-associated lipodystrophy.

JPRAS 2008; 61(4): 366-371

L Nelson, K.J. Stewart. Plastic surgical options for HIV-associated lipodystrophy. JPRAS 2008; 61(4): 359-365

L Nelson, K.J. Stewart 'HIV and the Surgeon' Invited editorial to accompany papers on HIV Lipodystrophy. JPRAS 2008; 61(4): 355-358

L. Nelson, Fried M, Stewart K.J. The risks of surgery in HIV-infected patients.

J Perioperative Practice 2007; 17(10):470-493

- Republished as Open Learning Zone article

L. Nelson, Fried M, Stewart K.J. HIV-infected patients: the risks of surgery J Perioperative Practice 2009; 19(1):24-30

L Nelson, K.J. Stewart 'Early and late complications of Polyalkylamide gel (Bio-Alcamid)®' JPRAS (published on-line July 2010)

\section{Book Chapter}

L. Nelson, K.J. Stewart 'Autologous fat transfer for HIV-associated facial lipoatrophy' Book Chapter in 'Surgical Facial Rejuvenation'.

Invited author 